\title{
Neurorehabilitation for upper
}

limb recovery after stroke

The use of non-invasive

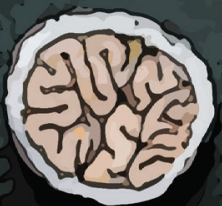
brain stimulation
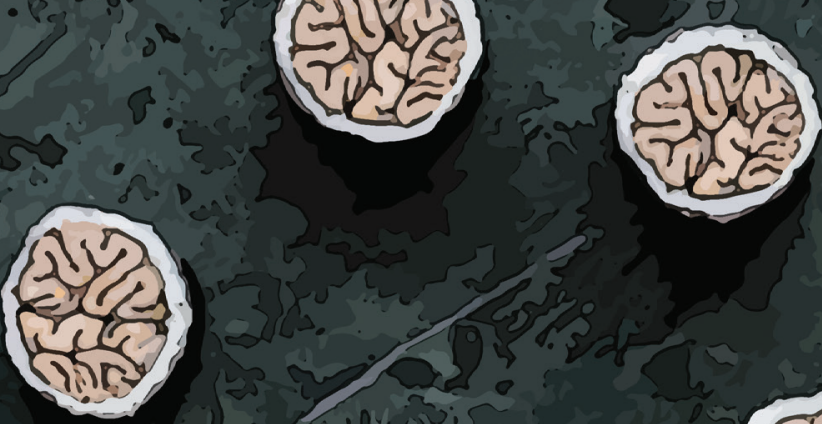

Eline van Lieshout 



\section{Neurorehabilitation for upper limb recovery after stroke}

The use of non-invasive brain stimulation

Eline van Lieshout 


$\begin{array}{ll}\text { Cover } & \text { Hugo Out } \\ \text { Layout } & \text { Renate Siebes | Proefschrift.nu } \\ \text { Printing } & \text { ProefschriftMaken, De Bilt } \\ \text { ISBN } & 978-90-393-7367-5\end{array}$

\section{(C) 2020 Eline van Lieshout}

All rights reserved. No part of this publication may be reproduced or transmitted in any form or by any means, electronically or mechanically, including photocopying, recording, or any information storage or retrieval system, without the prior permission of the author. 


\title{
Neurorehabilitation for upper limb recovery after stroke
}

The use of non-invasive brain stimulation

\author{
Neurorevalidatie voor armherstel na een beroerte \\ Het gebruik van niet-invasieve hersenstimulatie \\ (met een samenvatting in het Nederlands)
}

\section{Proefschrift}

ter verkrijging van de graad van doctor aan de Universiteit Utrecht op gezag van de rector magnificus, prof. dr. H.R.B.M. Kummeling, ingevolge het besluit van het college voor promoties in het openbaar te verdedigen op dinsdag 23 maart 2021 des middags te 2.30 uur

door

\section{Eline Christine Catharine van Lieshout}

geboren op 5 februari 1993

te Almere 
Promotoren:

Copromotor:
Prof. dr. R.M. Dijkhuizen

Prof. dr. J.M.A. Visser-Meily

Dr. H.B. van der Worp

The studies represented in this thesis were financially supported by the Netherlands Organisation for Scientific Research: VICI 016.130.662.

Financial support for the printing of this thesis has been generously provided by Stichting De Hoogstraat Onderzoeksfonds Utrecht, Brain Science Tools, Neurosoft, and InfoFiciency. 


\section{Contents}

Chapter 1 General introduction

PART I: Understanding and assessment of post-stroke impairment and recovery

Chapter 2 Translational value of skilled reaching assessment in clinical and preclinical studies on motor recovery after stroke

Chapter 3 Comparison of self-reported versus observational clinical measures of improvement in upper limb capacity in patients after stroke

Chapter 4 Does upper limb strength play a prominent role in healthrelated quality of life in stroke patients discharged from inpatient rehabilitation?

PART II: Aiming for optimal recovery - non-invasive brain stimulation in the rehabilitation of stroke

Chapter 5 Timing of repetitive transcranial magnetic stimulation onset for upper limb function after stroke: A systematic review and metaanalysis

Chapter 6 Brain stimulation for arm recovery after stroke (B-STARS): Protocol for a randomized controlled trial in subacute stroke patients

Chapter 7 Exploring the experiences of stroke patients treated with transcranial magnetic stimulation for upper limb recovery:

A qualitative study

Chapter 8 General discussion

Summary

Samenvatting (Summary in Dutch)

Dankwoord 
General introduction 

Stroke is one of the leading causes of acquired long-term disability in adults. ${ }^{1,2}$ Frequent consequences of stroke include cognitive, sensory and motor deficits, and additional problems such as depression, anxiety and fatigue. ${ }^{3,4}$ Acute stroke care has improved dramatically in the last two decades approximately with the introduction of intravenous thrombolysis and endovascular treatment, and has increased post-stroke survival rates. ${ }^{5,6}$ Yet, the global burden of stroke will significantly increase due to the ageing population and an increasing life expectancy in developed countries. ${ }^{7,8}$ Despite the advancements in acute stroke care, the absolute number of stroke survivors who never achieve complete restoration of function, and live with the consequences of stroke, is therefore increasing. 9,10

In most European countries, different institutions such as hospitals, geriatric rehabilitation, and rehabilitation centers are involved in the provision of stroke care. ${ }^{11,12}$ More than half of the stroke patients return home after acute stroke care in the hospital. Yearly, about one quarter of the hospitalized stroke patients is referred for rehabilitation in a specialized rehabilitation center or in a rehabilitation department in a general hospital, of which approximately one third starts as an inpatient. ${ }^{13}$ Older patients with multiple comorbidities are often referred to a geriatric rehabilitation center or to a chronic nursing facility. The main goal of stroke rehabilitation is to reduce the patient's disability and to improve participation and healthrelated quality of life. ${ }^{14}$

\section{Loss of arm function}

Physical disability after stroke is most often caused by sensorimotor impairments, which include a loss of strength and sensation in one or more extremities. ${ }^{10,14}$ Up to $85 \%$ of people with acute stroke experiences upper limb paresis, which is muscle weakness of the paretic arm. About half of the stroke patients with reduced arm function in the sub-acute phase after stroke still experiences functional deficits in the chronic phase. ${ }^{15}$ Impaired arm function is associated with low health-related quality of life (HRQOL), as it leads to difficulties with activities of daily living (ADL), such as reaching, picking up objects or using cutlery. ${ }^{16}$ Whilst some research has been carried out on the association between arm function and HRQoL, there has been no longitudinal investigation of changes in HRQoL in relation to improvement in arm recovery. The loss of arm function is often considered to be the most distressing long-term impairment for stroke patients. ${ }^{15,17,18}$

A qualitative study on the personal experience of upper limb recovery from the stroke survivor's perspective revealed that patients were in no doubt that getting going and keeping going with exercise was the physical means to keeping the door open for recovery. ${ }^{18}$ Unsurprisingly, identifying the best treatments for arm function recovery was therefore defined as a top-ten research priority relating to life after stroke by stroke survivors, 
care givers, and clinicians. ${ }^{19}$ For the above-mentioned reasons, we need to improve our understanding of upper limb recovery processes, develop optimized treatment strategies, and identify factors that predict motor outcomes. The predicted recovery of motor function influences decision-making regarding goals, type, and duration of rehabilitation for each patient.

\section{Stroke recovery}

Recovery can be described as improvement of body function and structure, activities, and participation. ${ }^{20}$ Stroke recovery occurs through a combination of restoration and compensation of functions. ${ }^{21}$ True functional recovery (i.e. restitution of function) refers to the process in which movement patterns change toward the original state before injury. In contrast, compensation means the use of alternative movements in order to replace a lost behavior. ${ }^{22,23}$ The interaction of post-stroke plasticity mechanisms (e.g. reorganization of neuronal networks, recruitment of functionally homologous pathways) and sensorimotor training can contribute to true recovery. Substitution is influenced by the use of explicit and implicit compensatory strategies. ${ }^{24}$ However, the distinction between recovery and compensation can rarely be made by traditional outcome measures, and compensatory movements may be mistaken for recovery. ${ }^{25}$

Most patients experience some degree of recovery of their lost motor function over time. ${ }^{26}$ The greatest improvements in upper limb function occur generally within the first three months after stroke due to spontaneous recovery and increased responsiveness to enriched environments and training (Figure 1.1). In this sub-acute phase spontaneous neurological recovery seems to follow a predictable pattern. This is expressed by the $70 \%$ proportional recovery rule, which states that the majority of the patients (recoverers) gain a fixed proportion (i.e. 70\%) of their potential recovery, measured with the Fugl-Meyer assessment of the upper extremity (FM-UE). ${ }^{27,28}$ Non-recoverers fail to follow this predictable pattern and do not show a significant amount of recovery. ${ }^{27-29}$ However, recently the $70 \%$ proportional recovery rule has been criticized for overestimating the predictability of the FM-UE.30 Predictions for arm function recovery are difficult to make for individuals, due to substantial interindividual variability (e.g. initial neurological deficit, absolute degree of reactive recovery), and this has led to a growing interest in biomarkers of motor recovery and outcomes. ${ }^{31-33}$ 


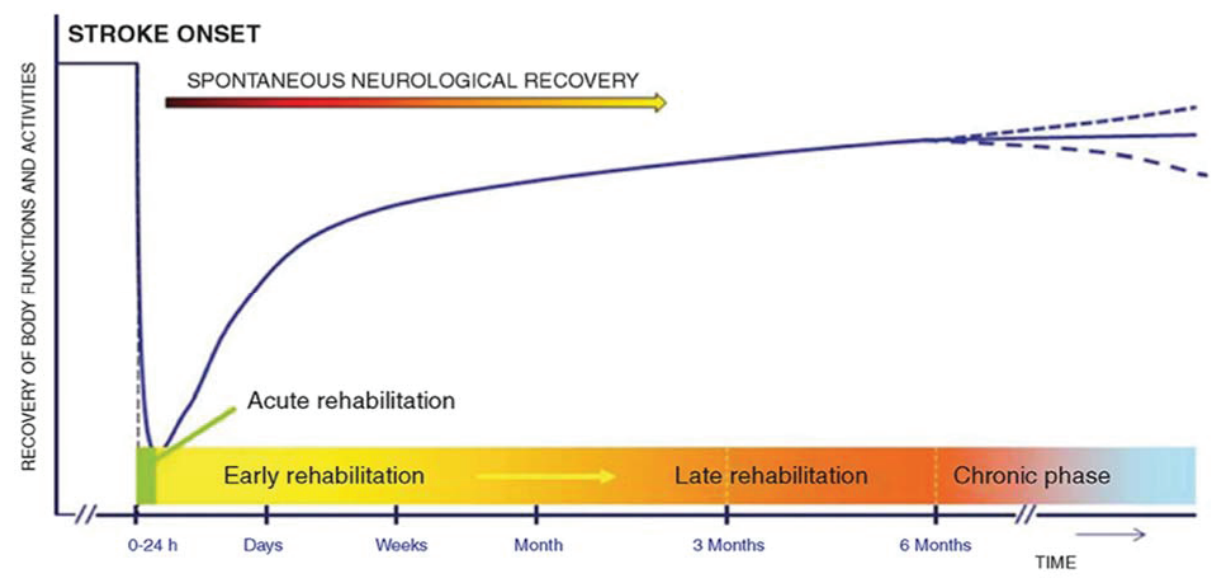

Figure 1.1: Hypothetical pattern of recovery after stroke over time.

Adapted from Kwakkel, Buma and Selzer (2014). ${ }^{34}$

\section{Predicting arm recovery and treatment effects}

Combining different biomarkers of motor recovery and outcomes (such as clinical, transcranial magnetic stimulation (TMS) and magnetic resonance imaging (MRI) measures) has shown to improve the accuracy of upper limb recovery predictions. ${ }^{30,35}$ Clinical measures of initial severity of motor impairment (e.g. from the FM-UE or Action Research Arm Test), ${ }^{36}$ neurophysiological measures of intactness of the corticospinal tract (e.g. from TMS), and neuroimaging biomarkers of structural integrity of white matter of descending white matter pathways (e.g. from diffusion-weighted MRI) have been related to motor outcomes. ${ }^{33}$ However, despite current advancements in recovery biomarkers, there is no consensus about which biomarkers have the highest predictive value for motor recovery. ${ }^{33}$ An accurate prognosis of an individual's potential for recovery, and an understanding of upper limb recovery patterns in association with post-stroke brain plasticity, would enable individual rehabilitation decisions. ${ }^{35}$

Knowledge of the upper limb recovery processes is essential for improving existing rehabilitation treatments and for developing new treatments, with the focus on optimizing therapy content, dose, duration, and delivery post-stroke. In general, the intervention should be of sufficiently high intensity and dose to induce the presumed neuroplastic changes that would underlie stroke recovery. ${ }^{37,38}$ Treatments should be task- and context-specific, ${ }^{14}$ and targeted towards the individual's goals to facilitate motivation and engagement in therapy. ${ }^{39}$ Despite the collective mindset 'time is brain' and early commencement of rehabilitation post-stroke in clinical practice guidelines, there is no intervention, besides revascularization through intravenous thrombolysis or endovascular treatment, that has proven to have a 
significant impact on spontaneous recovery. ${ }^{21,40}$ However, much is still unknown about the interaction between spontaneous recovery, the sensitive period for improvement, and (novel) rehabilitative interventions. ${ }^{21,29,41}$

\section{Non-invasive brain stimulation}

Different restorative therapies to enhance true recovery are currently under study: growth factors, stem cells, pharmacological compounds, activity- and cognitive-based training, robotics and brain computer interfaces, and brain stimulation. There has been a particularly growing interest in the use of non-invasive neuromodulation to enhance motor function recovery after stroke. ${ }^{42-45}$ Several studies have suggested that non-invasive brain stimulation (NIBS) promotes motor recovery of the upper limb, possibly through enhancement of motor cortex plasticity. ${ }^{32,46}$ NIBS techniques, such as repetitive TMS (rTMS) and transcranial direct current stimulation (tDCS), have the potential to increase or decrease cortical excitability, depending on the parameters of stimulation (Figure 1.2). ${ }^{47-49}$ High-frequency rTMS ( $\geq 3 \mathrm{~Hz}$ ) or intermittent theta burst stimulation (TBS), a specific rTMS stimulation protocol, can increase cortical excitability corresponding to long-term potentiation. Low-frequency $\operatorname{rTMS}(\leq 1 \mathrm{~Hz})$ or continuous TBS can decrease excitability corresponding to long-term depression. ${ }^{47,49,50}$ However, the effect sizes of therapeutic TMS are low and there is a high response variability between subjects receiving rTMS.$^{51}$ Factors like cortical thickness, activation history of the target hand muscle, and genetic variation are possible influencing determinants. ${ }^{47,50,52,53}$ Another NIBS technique, tDCS, which is also able to modulate cortical excitability, applies a weak electrical current (e.g. 1-2 mA) between two electrodes (anode and cathode) on the scalp, with the resulting current flow depending on the electrode placement and interaction between the two electrodes. ${ }^{54,55}$
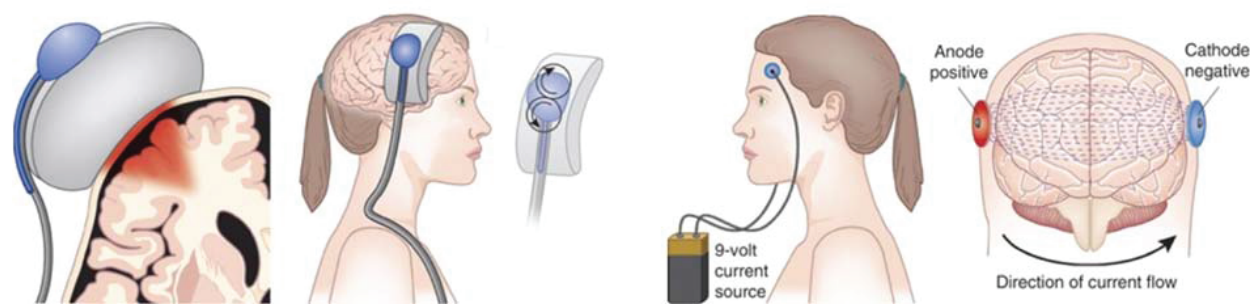

Figure 1.2: Non-invasive brain stimulation techniques: TMS (left) and tDCS (right). Adapted from George and Aston-Jones (2010). ${ }^{56}$ 
Until recently, the so-called interhemispheric imbalance model has been the basis for most NIBS protocols aimed at improving stroke recovery. ${ }^{57}$ This model assumes that after a stroke there is a reduced inhibition from the affected hemisphere to the unaffected hemisphere. The resulting increase in excitability of the unaffected hemisphere leads to excessive interhemispheric inhibition onto ipsilesional cortical areas. ${ }^{58-60}$ However, the validity of the interhemispheric imbalance model has been questioned, in particular for severely affected patients for whom contralesional areas may hold a compensatory role in motor recovery. ${ }^{61,62}$ An alternative model, based on vicariation, postulates that compensatory processes in homologous areas of the unaffected hemisphere lead to adaptive plasticity. ${ }^{62,63}$ Additionally, the bimodal balance recovery model unifies the opposite neuromodulatory approaches. ${ }^{57}$ If there is a high structural reserve (i.e. preserved integrity of the white matter motor pathways), over-activation of the unaffected hemisphere would be maladaptive, and then the interhemispheric inhibition model would dominate. If there is a limited structural reserve, over-activation could be compensatory, and thus the vicariation model would dominate. ${ }^{64}$ However, the validity of the models is yet to be determined, as which clinical and imaging-based biomarkers are reflective of these models and may be predictive of responses to NIBS in recovering stroke patients.

\section{rTMS treatment}

Earlier meta-analyses of small randomized controlled trials (RCTs) suggest that rTMS is able to transiently improve motor outcome of the paretic arm after stroke. By contrast, other studies failed to demonstrate long-lasting improvements in motor function. ${ }^{42,65,66}$ Thus, there are large differences between the results of RCTs, which could be explained by significant heterogeneity of patient population characteristics, intervention parameters, and selected outcome measures. ${ }^{67,68}$ Some studies demonstrated that the efficacy of rTMS depends on neural network connectivity. ${ }^{69,70} \mathrm{~A}$ recent RCT in 199 patients 3 to 12 months after stroke failed to demonstrate any beneficial effect of contralesional inhibitory stimulation paired with arm-motor training..$^{71}$ However, the lack of benefit may have been due to the initiation of treatment in the chronic phase post-stroke, as has been done in most studies. To date, little is known about the relation between the timing of rTMS after stroke and its efficacy. Currently, the implementation of interventions such as TMS in clinical practice remains low. Implementation of interventions is often limited by several barriers, such as unknown costeffectiveness, usability and acceptance by therapists and patients, and the way in which the effectiveness of the intervention has been evaluated. ${ }^{72}$ An investigation of patient's experiences and expectations of an intervention would be helpful to enhance successful implementation. 


\section{Upper limb outcome measures}

The evaluation of the effectiveness of upper limb treatments and the evaluation of patients' motor recovery progression are important aspects of clinical practice and (pre)clinical research. Clinicians and researchers use a wide range of measures to assess upper limb recovery, which limits the ability to detect the efficacy of an intervention through pooled analysis. ${ }^{73,74}$ Furthermore, in the translation of preclinical findings to clinical practice it is often neglected that true recovery and compensation are different. The Stroke Recovery and Rehabilitation Roundtable (SRRR) responded to the need of a standardized approach for measuring upper-limb recovery, both clinically and preclinically, and presented a core set of valid measures that should be used in every stroke recovery and rehabilitation trial and should be assessed at fixed time points post-stroke. ${ }^{75-77}$ It has been acknowledged that outcome measures should capture domains that are meaningful to patients and enable them to recognize their (improved) performance, for example by the use of patient-reported outcome measures. ${ }^{78}$ As the time course of upper limb recovery is non-linear and is driven by poorly understood processes of neural recovery and compensation strategies, outcomes assessed by researchers or physicians and those reported by patients themselves may differ, also depending on the timing of assessment post-stroke.

\section{Aim and outline of the thesis}

The overall aim of the thesis is to provide a perspective on the understanding and assessments of post-stroke impairment and recovery, and on the potential of NIBS techniques, particularly TMS, to improve upper limb performance.

The thesis is divided in two parts. The first part reports on the results of three studies on the assessment of upper limb recovery, of which the first is a translational study on upper limb recovery patterns in the context of skilled reaching (i.e. reach-to-eat movement) in rodents and humans, and their relation with clinical outcomes (Chapter 2). The second study focuses on observational versus self-reported clinical measures for upper limb capacity (Chapter 3), while the third study deals with the role of upper limb strength in HRQOL in stroke patients discharged from inpatient rehabilitation (Chapter 4). The second part of the thesis outlines the available evidence of efficacy of NIBS techniques in post-stroke motor rehabilitation, based on a systematic review and meta-analysis (Chapter 5 ). The second part also describes the study protocol of an RCT on therapeutic rTMS to improve upper limb function in patients in the first weeks after stroke (Chapter 6), and it reports results from a qualitative study that evaluated the experiences of patients who participated in this RCT (Chapter 7). The thesis ends with a general discussion of the main results and methodological considerations, and it includes several recommendations for clinical practice and future research (Chapter 8). 


\section{References}

1. Mendis, S. Stroke disability and rehabilitation of stroke: World Health Organization perspective. Int. J. Stroke 8, 3-4 (2013).

2. Kyu, H. H. et al. Global, regional, and national disability-adjusted life-years (DALYs) for 359 diseases and injuries and healthy life expectancy (HALE) for 195 countries and territories, 1990-2017: A systematic analysis for the Global Burden of Disease Study 2017. Lancet 392, 1859-1922 (2018).

3. Morris, J. H., Van Wijck, F., Joice, S. \& Donaghy, M. Predicting health related quality of life 6 months after stroke: The role of anxiety and upper limb dysfunction. Disabil. Rehabil. 35, 291-299 (2013).

4. Bernhardt, J. et al. Agreed definitions and a shared vision for new standards in stroke recovery research : The Stroke Recovery and Rehabilitation Roundtable taskforce. Int. J. Stroke 12, 444-450 (2017).

5. Asadi, H., Williams, D. \& Thornton, J. Changing management of acute ischaemic stroke: The new treatments and emerging role of endovascular therapy. Curr. Treat. Options Neurol. 18, (2016).

6. Hankey, G. J. Stroke. The Lancet 389, 641-654 (2017).

7. Dis, I., Buddeke, J., Vaartjes, I., Visseren, F. \& Bots, M. Hart- en vaatziekten in Nederland 2015, cijfers over heden, verleden en toekomst. Ned. Harts. 132 (2015).

8. Feigin, V. et al. Update on the Global Burden of Ischaemic and Haemorrhagic Stroke in 1990-2013: The GBD 2013 Study. Neuroepidemiology 45, 161-176 (2016).

9. Go, A. et al. Heart Disease and Stroke Statistics-2013 Update: A Report From the American Heart Association. Circulation. 127, e36-e245 (2013).

10. Broeks, J. G., Lankhorst, G. J., Rumping, K. \& Prevo, A. J. The long-term outcome of arm function after stroke: results of a follow-up study. Disabil. Rehabil. 21, 357-364 (1999).

11. Groeneveld, I. F. et al. Practice variation in the structure of stroke rehabilitation in four rehabilitation centres in the Netherlands. J. Rehabil. Med. 48, 287-292 (2016).

12. Grund, S. et al. European consensus on core principles and future priorities for geriatric rehabilitation: consensus statement. Eur. Geriatr. Med. 11, 233-238 (2019).

13. de Boer, A. R., van Dis, I., Vissseren, F. L. J., Vaartjes, I. \& Bots, M. L. Hart- en vaatziekten in Nederland 2018, cijfers over risicofactoren, hartinterventies, ziekte en sterfte. Den Haag: Hartstichting (2018).

14. Langhorne, P., Bernhardt, J. \& Kwakkel, G. Stroke rehabilitation. Lancet 377, 1693-1702 (2011).

15. Kwakkel, G., Kollen, B. J., Van der Grond, J. V. \& Prevo, A. J. H. Probability of regaining dexterity in the flaccid upper limb: Impact of severity of paresis and time since onset in acute stroke. Stroke 34, 2181-2186 (2003).

16. Franceschini, M., La Porta, F., Agosti, M. \& Massucci, M. Is health-related-quality of life of stroke patients influenced by neurological impairments at one year after stroke? Eur. J. Phys. Rehabil. Med. 46, 389-399 (2010).

17. Lai, S. M., Studenski, S., Duncan, P. W. \& Perera, S. Persisting consequences of stroke measured by the stroke impact scale. Stroke 33, 1840-1844 (2002).

18. Barker, R. N., Gill, T. J. \& Brauer, S. G. 'Factors contributing to upper limb recovery after stroke: A survey of stroke survivors in Queensland Australia'. Disabil. Rehabil. 29, 981-989 (2007).

19. Pollock, A., St George, B., Fenton, M. \& Firkins, L. Top ten research priorities relating to life after stroke. Lancet Neurol. 11, 209 (2012).

20. World Health Organization (WHO). International classification of functioning, disability and health. World Health Organization 1-15 (2003). Available at: http://www.who.int/classifications/icf/ icfchecklist.pdf?ua=1.

21. Zeiler, S. R. Should We Care About Early Post-Stroke Rehabilitation? Not Yet, but Soon. Curr. Neurol. Neurosci. Rep. 19, (2019). 
22. Levin, M. F., Kleim, J. A. \& Wolf, S. L. What Do Motor Recovery and Compensation Mean in Patients Following Stroke? Neurorehabil. Neural Repair 23, 313-319 (2008).

23. Nijland, R. et al. Characterizing the Protocol for Early Modified Constraint-induced Movement Therapy in the EXPLICIT-Stroke Trial. Physiother. Res. Int. 18, 1-15 (2013).

24. Dancause, N. \& Nudo, R. J. Shaping plasticity to enhance recovery after injury. Prog Brain Res. 192, 273-295 (2011).

25. Alaverdashvili, M. \& Whishaw, I. Q. A behavioral method for identifying recovery and compensation: Hand use in a preclinical stroke model using the single pellet reaching task. Neurosci. Biobehav. Rev. 37, 950-967 (2013).

26. Schaechter, J. D. Motor rehabilitation and brain plasticity after hemiparetic stroke. Prog. Neurobiol. 73, 61-72 (2004).

27. Krakauer, J. W. \& Marshall, R. S. The proportional recovery rule for stroke revisited. Ann. Neurol. 78, 845-847 (2015).

28. Winters, C., Van Wegen, E. E. H., Daffertshofer, A. \& Kwakkel, G. Generalizability of the Proportional Recovery Model for the Upper Extremity After an Ischemic Stroke. Neurorehabil. Neural Repair 29, 614-622 (2015).

29. Prabhakaran, S. et al. Inter-individual variability in the capacity for motor recovery after ischemic stroke. Neurorehabil. Neural Repair 22, 64-71 (2008).

30. Vliet, R. et al. Predicting upper limb motor impairment recovery after stroke: a mixture model. Ann. Neurol. 87, 383-393 (2020).

31. Stinear, C. M. Prediction of motor recovery after stroke: advances in biomarkers. Lancet Neurol. 16, 826-836 (2017).

32. Bernhardt, J. et al. Moving rehabilitation research forward: Developing consensus statements for rehabilitation and recovery research. Int. J. Stroke 11, 454-458 (2016).

33. Kim, B. \& Winstein, C. Can Neurological Biomarkers of Brain Impairment Be Used to Predict Poststroke Motor Recovery? A Systematic Review. Neurorehabil. Neural Repair 31, 3-24 (2017).

34. Selzer, M. E., Clarke, S., Cohen, L., Duncan, P. \& Gage, F. Understanding mechanisms underlying recovery after stroke. in Textbook of Neural Repair and Rehabilitation (Cambridge University Press, 2014). doi:10.1017/cbo9780511995590

35. Stinear, C. M., Barber, P. A., Petoe, M., Anwar, S. \& Byblow, W. D. The PREP algorithm predicts potential for upper limb recovery after stroke. Brain 135, 2527-2535 (2012).

36. Coupar, F., Pollock, A., Rowe, P., Weir, C. \& Langhorne, P. Predictors of upper limb recovery after stroke: A systematic review and meta-analysis. Clin. Rehabil. 26, 291-313 (2012).

37. Lohse, K. R., Lang, C. E. \& Boyd, L. A. Is more better? Using metadata to explore dose-response relationships in stroke rehabilitation. Stroke 45, 2053-2058 (2014).

38. Ward, N. S., Brander, F. \& Kelly, K. Intensive upper limb neurorehabilitation in chronic stroke: Outcomes from the Queen Square programme. J. Neurol. Neurosurg. Psychiatry 90, 498-506 (2019).

39. Luker, J., Lynch, E., Bernhardsson, S., Bennett, L. \& Bernhardt, J. Stroke Survivors' Experiences of Physical Rehabilitation: A Systematic Review of Qualitative Studies. Arch. Phys. Med. Rehabil. 96, 1698-1708.e10 (2015).

40. Bernhardt, J., English, C., Johnson, L. \& Cumming, T. B. Early mobilization after stroke: early adoption but limited evidence. Stroke 46, 1141-1146 (2015).

41. Heredia, M. et al. Motor improvement of skilled forelimb use induced by treatment with growth hormone and rehabilitation isdependent on the onset of the treatment after cortical ablation. Neural Plast. 2018, 6125901 (2018). 
42. Hsu, W. Y., Cheng, C. H., Liao, K. K., Lee, I. H. \& Lin, Y. Y. Effects of repetitive transcranial magnetic stimulation on motor functions in patients with stroke: A meta-analysis. Stroke 43, 1849-1857 (2012).

43. Scheidtmann, K., Fries, W., Müller, F. \& Koenig, E. Effect of levodopa in combination with physiotherapy on functional motor recovery after stroke: A prospective, randomised, double-blind study. Lancet 358, 787-790 (2001).

44. Sale, P. et al. Electroencephalographic markers of robot-aided therapy in stroke patients for the evaluation of upper limb rehabilitation. Int. J. Rehabil. Res. 38, 294-305 (2015).

45. Könönen, M. et al. Functional MRI and motor behavioral changes obtained with constraint-induced movement therapy in chronic stroke. Eur. J. Neurol. 19, 578-586 (2012).

46. Sebastianelli, L. et al. Low-frequency rTMS of the unaffected hemisphere in stroke patients: A systematic review. Acta Neurol. Scand. 136, 585-605 (2017).

47. Kubis, N. Non-Invasive Brain Stimulation to Enhance Post-Stroke Recovery. Front. Neural Circuits 10, 56 (2016).

48. Chung, S. W., Hill, A. T., Rogasch, N. C., Hoy, K. E. \& Fitzgerald, P. B. Use of theta-burst stimulation in changing excitability of motor cortex: A systematic review and meta-analysis. Neurosci. Biobehav. Rev. 63, 43-64 (2016).

49. Klomjai, W., Katz, R. \& Lackmy-Vallée, A. Basic principles of transcranial magnetic stimulation (TMS) and repetitive TMS (rTMS). Ann. Phys. Rehabil. Med. 58, 208-213 (2015).

50. Goldsworthy, M. R., Müller-Dahlhaus, F., Ridding, M. C. \& Ziemann, U. Inter-subject variability of LTD-like plasticity in human motor cortex: A matter of preceding motor activation. Brain Stimul. 7, 864-870 (2014).

51. Ziemann, U. Thirty years of transcranial magnetic stimulation: where do we stand? Exp. Brain Res. 235, 973-984 (2017).

52. Conde, V. et al. Cortical thickness in primary sensorimotor cortex influences the effectiveness of paired associative stimulation. Neuroimage 60, 864-870 (2012).

53. Cheeran, B. et al. A common polymorphism in the brain-derived neurotrophic factor gene (BDNF) modulates human cortical plasticity and the response to rTMS. J. Physiol. 586, 5717-5725 (2008).

54. Nitsche, M. A. \& Paulus, W. Sustained excitability elevations induced by transcranial DC motor cortex stimulation in humans. Neurology 57, 1899-901 (2001).

55. Nitsche, M. A. \& Paulus, W. Excitability changes induced in the human motor cortex by weak transcranial direct current stimulation. J. Physiol. 527 Pt 3, 633-9 (2000).

56. George, M. S. \& Aston-Jones, G. Noninvasive techniques for probing neurocircuitry and treating illness: Vagus nerve stimulation (VNS), transcranial magnetic stimulation (TMS) and transcranial direct current stimulation (tDCS). Neuropsychopharmacology 35, 301-316 (2010).

57. Di Pino, G. et al. Modulation of brain plasticity in stroke: a novel model for neurorehabilitation. Nat. Rev. Neurol. 10, 597-608 (2014).

58. Ovadia-Caro, S. et al. Predicting the response to noninvasive brain stimulation in stroke. Front. Neurol. 10, 1-6 (2019).

59. Murase, N., Duqye, J., Mazzocchio, R. \& Cohen, L. G. Influence of interhemispheric interactions on motor function in chronic stroke. Ann. Neurol. 55, 400-409 (2004).

60. Liepert, J., Hamzei, F. \& Weiller, C. Motor cortex disinhibition of the unaffected hemisphere after acute stroke. Muscle and Nerve 23, 1761-3 (2000).

61. Bestmann, S. et al. The role of contralesional dorsal premotor cortex after stroke as studied with concurrent TMS-fMRI. J. Neurosci. 30, 11926-37 (2010).

62. Johansen-Berg, $\mathrm{H}$. et al. The role of ipsilateral premotor cortex in hand movement after stroke. Proc. Natl. Acad. Sci. U. S. A. 30, 14518-23 (2002). 
63. Frost, S. B., Barbay, S., Friel, K. M., Plautz, E. J. \& Nudo, R. J. Reorganization of remote cortical regions after ischemic brain injury: A potential substrate for stroke recovery. J. Neurophysiol. 89, 3205-2314 (2003).

64. Di Pino, G. et al. Modulation of brain plasticity in stroke: a novel model for neurorehabilitation. Nat. Rev. Neurol. 10, 597-608 (2014).

65. Le, Q., Qu, Y., Tao, Y. \& Zhu, S. Effects of repetitive transcranial magnetic stimulation on hand function recovery and excitability of the motor cortex after stroke: A meta-analysis. Am. J. Phys. Med. Rehabil. 93, 1-9 (2014).

66. Adeyemo, B. O., Simis, M., Macea, D. D. \& Fregni, F. Systematic review of parameters of stimulation, clinical trial design characteristics, and motor outcomes in non-invasive brain stimulation in stroke. Front. Psychiatry 3, 1-27 (2012).

67. Graef, P., Dadalt, M. L. R., da Silva Rodrigués, D. A. M., Stein, C. \& de Souza Pagnussat, A. Transcranial magnetic stimulation combined with upper-limb training for improving function after stroke: A systematic review and meta-analysis. J. Neurol. Sci. 369, 149-158 (2016).

68. Hao, Z., Wang, D., Zeng, Y. \& Liu, M. Repetitive transcranial magnetic stimulation for improving function after stroke. Cochrane Libr. 5, (2013).

69. Liston, C. et al. Default mode network mechanisms of transcranial magnetic stimulation in depression. Biol. Psychiatry 76, 517-526 (2014).

70. Salomons, T. V. et al. Resting-state cortico-thalamic-striatal connectivity predicts response to dorsomedial prefrontal rTMS in major depressive disorder. Neuropsychopharmacology 39, 488-498 (2014).

71. Harvey, R. L. et al. Randomized Sham-Controlled Trial of Navigated Repetitive Transcranial Magnetic Stimulation for Motor Recovery in Stroke. Stroke 49, 2138-2146 (2018).

72. Hochstenbach-Waelen, A. \& Seelen, H. A. M. Embracing change: Practical and theoretical considerations for successful implementation of technology assisting upper limb training in stroke. J. Neuroeng. Rehabil. 9, 52 (2012).

73. Santisteban, L. et al. Upper limb outcome measures used in stroke rehabilitation studies: A systematic literature review. PLoS One 11, e0154792 (2016).

74. Ali, M., English, C., Bernhardt, J., Sunnerhagen, K. S. \& Brady, M. More outcomes than trials: A call for consistent data collection across stroke rehabilitation trials. Int. J. Stroke 8, 18-24 (2013).

75. Kwakkel, G. Standardised measurement of sensorimotor recovery in stroke trials: consensus-based core recommendations from the Stroke Recovery and Rehabilitation Roundtable (SRRR). Int. J. Stroke 12, 451-461 (2017).

76. Duncan Millar, J., van Wijck, F., Pollock, A. \& Ali, M. Outcome measures in post-stroke arm rehabilitation trials: do existing measures capture outcomes that are important to stroke survivors, carers, and clinicians? Clin. Rehabil. 33, 737-749 (2019).

77. van Lieshout, E. C. C., van der Worp, H. B., Visser-Meily, J. M. A. \& Dijkhuizen, R. M. Timing of Repetitive Transcranial Magnetic Stimulation Onset for Upper Limb Function After Stroke: A Systematic Review and Meta-Analysis. Front. Neurol. 10, 1-16 (2019).

78. Stewart, J. C. \& Cramer, S. C. Patient-reported measures provide unique insights into motor function after stroke. Stroke 44, 1111-1116 (2013). 

ث̊

$\operatorname{lol}^{2}$ renticas

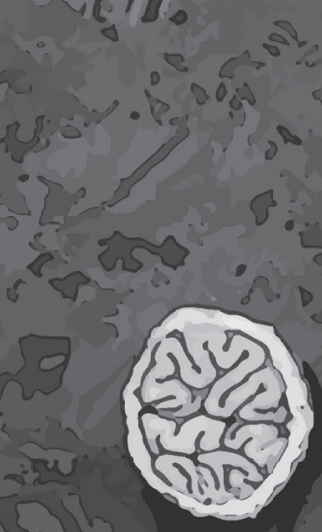

(1) ब्रा

(1)
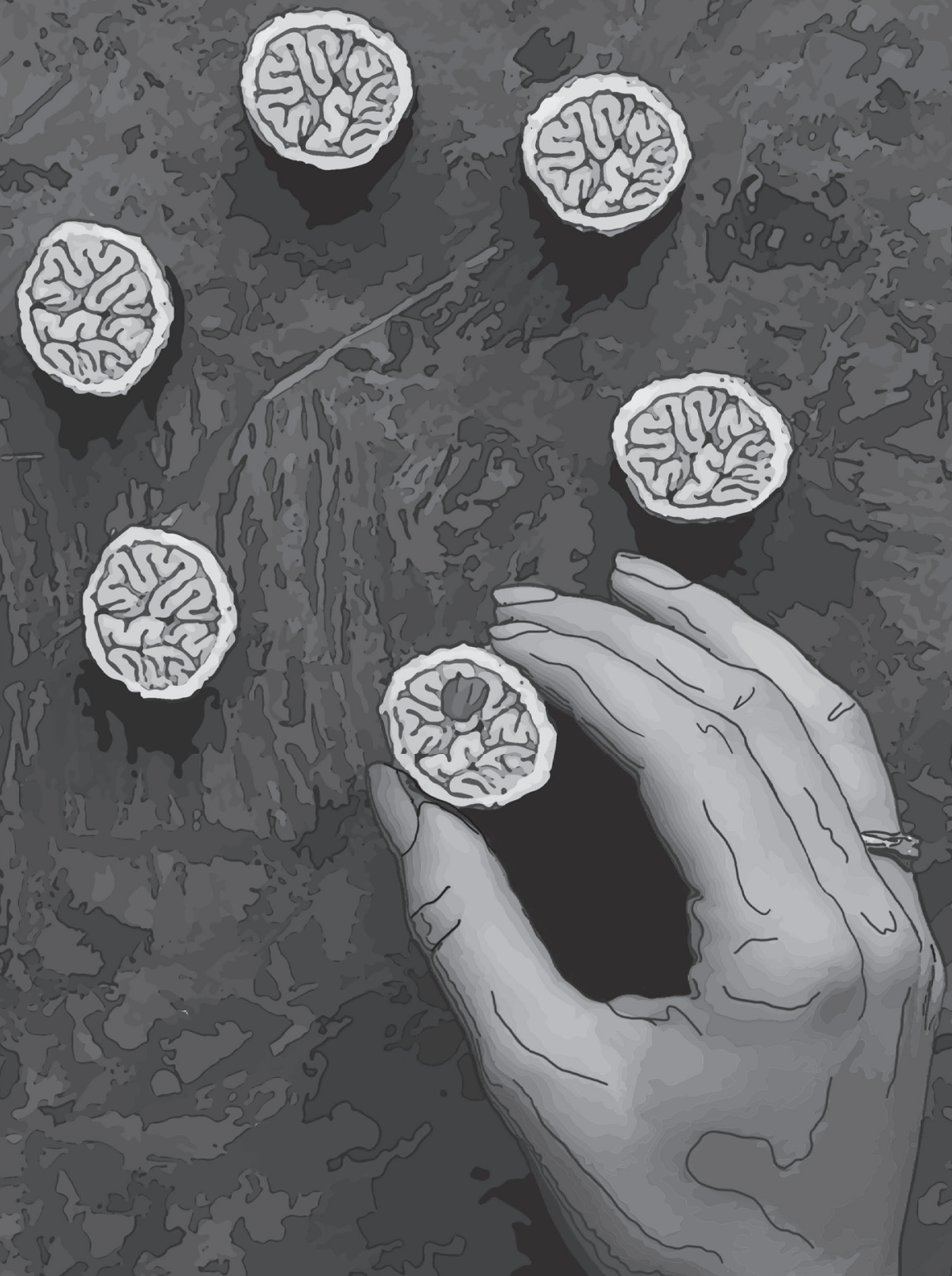


\section{Translational value of skilled reaching assessment in clinical and preclinical studies on motor recovery after stroke}

Eline C.C. van Lieshout Julia Boonzaier Adam J. Pel

Caroline L. van Heijningen Jord J. Vink Johanna M.A. Visser-Meily

Geralda A. van Tilborg Rick M. Dijkhuizen 


\section{Abstract}

Background: Assessment of skilled reaching enables extensive analysis of upper limb function in clinical and preclinical studies on post-stroke outcome. However, translational research if often limited by lack of correspondence between tests of human and rodent motor function.

Objectives: To determine 1) the translational value of skilled reaching performance for preclinical research by comparing the behavioural recovery profiles of skilled reaching characteristics between humans and rats recovering from stroke, and 2) the relationship between skilled reaching performance and commonly used clinical outcome measures after stroke.

Methods: Twelve patients with ischemic or hemorrhagic stroke and seventeen rats with photothrombotic stroke underwent an equivalent skilled reaching test at different timepoints, representing early to late sub-acute stages post-stroke. Success scores and a movement element rating scale were used to measure the skilled reaching performance. The Fugl-Meyer Upper Extremity (FM-UE) assessment and the Action Research Arm Test (ARAT) were used as clinical outcome measures.

Results: Both species had muscle flaccidity at the early sub-acute stage after stroke and showed motor recovery following a proximal-distal principle towards the early sub-acute stage, albeit for rats within a shorter time-course. Human skilled reaching scores and FM-UE and ARAT scores in the first three months post-stroke were significantly correlated $(p<.05)$.

Conclusions: Our study demonstrates that post-stroke changes in skilled reaching performance are highly similar between rats and humans, and correspond with standard clinical outcome measures. Skilled reaching testing therefore offers an effective and highly translational means for assessment of motor recovery in experimental and clinical stroke settings. 


\section{Introduction}

Upper limb impairment, such as a hemiparesis of the contralateral limb, is diagnosed in about $75 \%$ of the stroke patient population. ${ }^{1,2}$ Upper limb impairment limits functional independence, participation in social roles, and a return to work. ${ }^{3}$ Impairments in skilled use of the hands are cardinal features of post-stroke motor dysfunction, ${ }^{4}$ and compensatory movement patterns, e.g. excessive trunk displacement and increased reliance on the nonparetic hand, are common responses to hand function loss. ${ }^{5}$

Compensation is often mistaken for recovery, since some compensatory movement patterns are subtle enough to be undetected in clinical outcome measures that focus little on qualitative aspects of movement. ${ }^{6}$ In the process of post-stroke motor recovery, early adoption of compensation strategies may lead to learned disuse or training-induced misuse of the impaired limb, which in the long term can limit a patient's rehabilitation. ${ }^{7}$ In addition, when recovery is evaluated without taking compensation into account, this could distort understanding of the contribution of neural plasticity to post-stroke recovery. This is particularly relevant in basic neuroscience studies and translational research on spontaneous recovery or restorative treatments, ${ }^{6,7}$ which often make use of animal models, mostly involving rodents. However, translational research is often limited by lack of correspondence between tests of human and rodent motor function. ${ }^{8,9}$ Skilled reaching assessment has been proposed as one of the most potent translational behavioral tests for studying post-stroke recovery in rodents. ${ }^{9}$ The typical task requires that a subject reaches for and subsequently grasps a small food item with a single hand/paw, and subsequently brings it to the mouth for eating. ${ }^{10}$ Skilled reaching (conventional term for reach-to-eat) movement patterns show significant homologies between rodents and humans, ${ }^{11-13}$ which offers valuable opportunities for translational research.

Guidelines to enhance the alignment of preclinical and clinical stroke recovery research pipeline have recently been published by the Stroke Recovery and Rehabilitation Roundtable (SRRR) consortium. ${ }^{8}$ Behavioural outcome measures have received special consideration and it has been recommended that clinically relevant deficits, such as skilled reaching should be the main focus of preclinical behavioural testing. ${ }^{14}$ The time-course of recovery is more rapid in rodents than in humans, ${ }^{14,15}$ and it remains to be determined to what extent behavioural recovery profiles in rodent stroke models are representative for functional recovery patterns in stroke patients. To our knowledge, the development of skilled reaching performance over time after stroke has not yet been directly compared between rats and humans. Therefore, the aim of our study was to determine the degree of correspondence of temporal changes in several skilled reaching characteristics in rats and humans between early and late subacute stages post-stroke. To further evaluate the translational value of skilled reaching, our second aim was to determine the relationship between skilled reaching 
performance and commonly used clinical outcome measures (i.e. Fugl-Meyer assessment and Action Research Arm Test scores) in subacute stroke patients.

\section{Methods}

\section{Stroke patients}

Human data for this study was collected from the B-STARS trial, which assesses the effect of repetitive transcranial magnetic brain stimulation (rTMS) on upper limb recovery after stroke. ${ }^{16}$ Patients were included from whom complete skilled reaching, FM-UE and ARAT data was available for the first three months after stroke. The B-STARS trial, which was ongoing at the time of submission of the current manuscript, is a stratified, randomized controlled trial consisting of a two-week rTMS or sham-stimulation treatment starting at 2-3 weeks post-stroke. Twelve patients from both treatment groups were included, and investigators were blinded for group assignments. Patients with completed skilled reaching assessments between November 2018 and November 2019 were included. At seven time points, multiple performance assays and functional tasks have been conducted to monitor upper limb recovery, among which a skilled reaching task, Fugl-Meyer Upper Extremity assessment (FM-UE) and the action research arm test (ARAT) (details described below). Outcomes from assessment at 2-3 weeks (before intervention), 4-5 weeks (28-35 days) (no FM-UE assessment), 6 weeks (40-44 days), 9 weeks (59-67 days), and 11-15 weeks (76-104 days) post-stroke were used for the current study. All patients underwent MRI sessions at 5-6 weeks, 11-15 weeks, 22-26 weeks, and/or 46-50 weeks (see Appendix 2.1). All patients were inpatients of a rehabilitation facility in the Netherlands. The inpatient rehabilitation program consisted of a multidisciplinary approach to reach complex (physical and cognitive) rehabilitation goals. Full details of the B-STARS trial have been reported elsewhere. ${ }^{16}$

The B-STARS protocol has been approved by the Medical Ethics Committee of the University Medical Center Utrecht and the participating rehabilitation centre. The trial is registered in the Dutch Trial Register (Trial NL5952). All patients gave (written) informed consent to participate in the trial.

\section{Rat stroke model}

Animal data was collected from a randomized controlled pre-clinical trial, which was ongoing at the time of submission of the current manuscript, to assess the effects of rTMS (versus sham stimulation) on forelimb recovery after unilateral photothrombotic stroke in the forelimb region of the sensorimotor cortex. Rats from both treatment groups were included, and investigators were blinded for group assignments. Details on the photothrombotic stroke induction and 
rTMS protocols can be found in Appendix 2.1. Seventeen male Sprague Dawley rats (Charles River Laboratories, Sulzfeld, Germany) (326, \pm 26 g, 10 weeks old at the time of stroke induction) underwent skilled reaching tests (details described below) at days 0 (pre-stroke), 3 , 9,16 , and 23 post-stroke. Animals underwent MRI, which included anatomical MRI for lesion detection (see Appendix 2.1) at 2, 17 and 24 days post-stroke. All experiments were approved by the Animal Ethics Committee of the University Medical Center Utrecht, The Netherlands, and were conducted in agreement with Dutch laws ("Wet op de Dierproeven", 1996) and European regulations (Guideline 86/609/EEC).

The animals were housed under a regular 12-hour light/dark cycle and at constant temperature $\left(24^{\circ} \mathrm{C}, 45-65 \%\right.$ humidity). Prior to the stroke induction surgery, the rats underwent skilled reaching training and were housed per 2 or 3 in standard cages $\left(30 \times 40 \times 20 \mathrm{~cm}^{3}\right)$. During skilled reaching training (described in Appendix 2.1), the animals were food-restricted to reach $90 \%$ of their initial body weight by receiving $26-30 \mathrm{~g}$ of food per cage (daily), with water freely available.

Three days after stroke induction, the animals were moved to an enriched environment, as a standard procedure, representing a clinical rehabilitation setting. ${ }^{17}$ While the animals were housed in groups of 4-5 in the enriched environment, they were only food-deprived during the dark cycle before a skilled reaching test.

\section{Skilled reaching task - humans}

Stroke patients were seated in a chair, with their feet flat on the ground and hands palm down on their thighs with the fingers extended. A small food item (Kellogg's ${ }^{\circledR}$ Honey Pops Loops) was placed on a pedestal positioned in front of the patient, adjusted to their trunk height and arm length at full extension $(10 \mathrm{~cm}$ beneath the outstretched palm) (see Figure 2.1A). The patient was asked, in a standardized way by the researcher, to reach for the food item, grasp it, and place it into the mouth for eating. The skilled reaching task was performed once for the non-paretic side and at least once (out of maximally five attempts) for the paretic side at each time-point.

\section{Skilled reaching task - rats}

Rats were placed in a rectangular skilled reaching box made of transparent Plexiglas (Figure 2.1B). The animals were trained to grasp (at least 20) sugar pellets through a vertical slot ( $1 \mathrm{~cm}$ wide, extended $3 \mathrm{~cm}$ above the floor) in the front wall of the box. On the outside of the wall, in front of the slot, mounted $3 \mathrm{~cm}$ above the floor, was a shelf with an indentation allowing pellet placement slightly lateral to the opening of the slot. This off-center positioning of the pellet forced rats to use their dominant/preferred forelimb (determined 


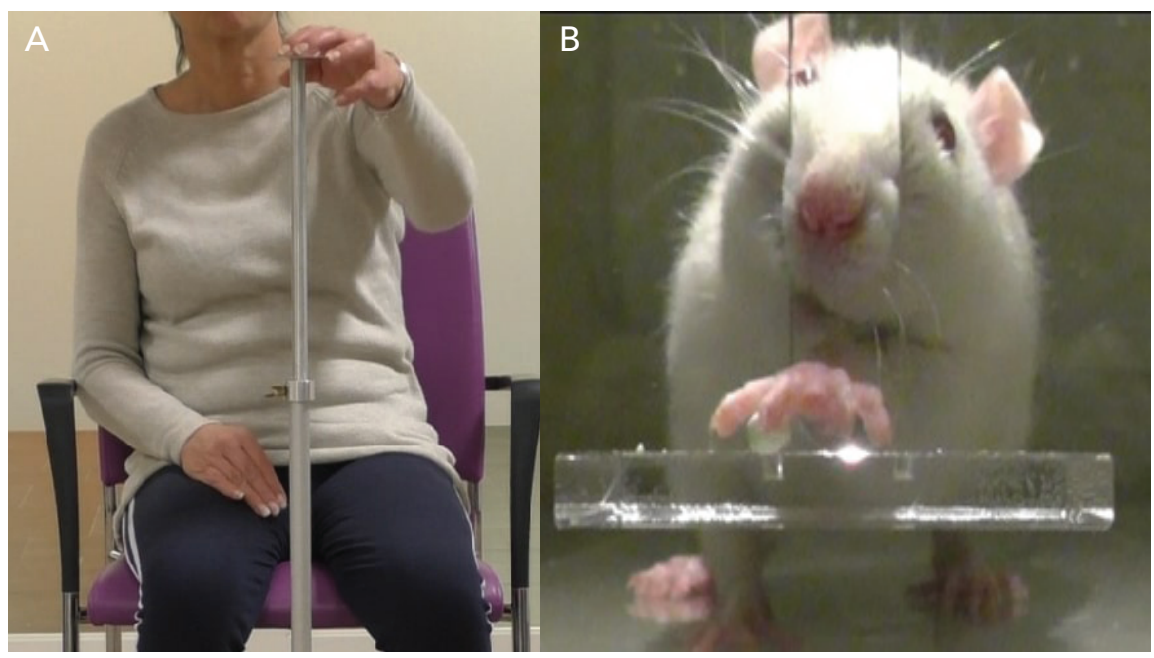

Figure 2.1: Set-up of the skilled reaching task for humans (A) and rats (B) (single frontal image from video recording).

during the training phase) to obtain the target. For the study, the rats executed 20 trials. However, in some cases (21\% of all sessions) less trials were executed due to impairment or motivation issues. Details on the skilled reaching (schedule) and food restriction protocols can be found in Appendix 2.1.

\section{Video recording}

High-speed video recording from the frontal perspective was used for humans (Panasonic HC-V770) and rats (Panasonic HC-V520) with a shutter speed set at 1/1000 frames per second, to produce a blur-free image for frame-by-frame playback (see Figure 2.1). For recordings of skilled reaching in rats, two specific light sources were used.

The recordings were analyzed using VLC media player (VideoLAN, Paris, France). Analyses of the human and rodent data were performed independently by a clinical (AJP) and preclinical researcher $(\mathrm{CLVH})$, respectively.

\section{Skilled reaching performance}

Reaching behavior was analyzed 1) from the end-point measure of success, and 2) with a movement element scoring system. A reach was defined as a success when the food item was grasped, transported by the hand or paw, and placed into the mouth.

Success percentage was expressed as the number of subjects (patients or rats) that could execute a successful, fully completed reach (i.e. food item was grasped, transported by 
the hand or paw, and placed into the mouth), divided by the total number of subjects, for each individual time-point. This adapted measure of successful reaching was used for comparison across species. Stroke patients executed the reaching task up to maximally five times, to limit the time and the impact of fatigue and frustration associated with repetitive task executions and recurrent failures, respectively. For rats, the conventional measure of success rate, i.e. the percentage of successfully obtained pellets with respect to the number of trials, was also calculated. ${ }^{18}$

Skilled reaching performance was scored using a biometric rating scale, based on a conceptual framework derived from the Eshkol-Wachmann Movement Notation (EWMN). ${ }^{18}$ The EWMN describes the position of the individual limbs, the trunk, the snout (rat), and the head in relation to the body or the food (pellet), with the body treated as a system of axes (i.e. limb segments, trunk axis, and snout axis (rat)). For stroke patients, the best attempt (fully or furthest completed) with the paretic limb was selected for movement analysis with the EshkolWachmann Movement Notation-Derived Reaching Scale (EW-DRS). This scale is divided into seven elements: orient, lift, advance, pronate, grasp, supinate, and release. Each element is described with regards to its proper execution, e.g. "initial hand lift is due to flexion of the elbow", and "trunk leans to the side opposite to the reach as hand approaches the target". 4,9 The elements were further divided into two or more sub-elements that are rated on a 3-point ordinal scale, from 0 (movement was normal) to 0.5 (movement was present but abnormal or incomplete) to 1 (movement was absent). Elements that could not be executed, were scored as no movement (i.e. score of 1). The overall score was the sum of all subelement scores (possible range: 0 to 21), with lower scores representing better performance. Sum scores were converted to a 0-1 scale by dividing by the maximum score, to allow direct comparison between the movement elements and against corresponding rat data. For each rat, the three best reaches (fully or furthest completed) for each session were scored, averaged and selected for further movement analysis with an EW-DRS adapted to Sprague-Dawley rats. ${ }^{19}$ Eleven elements of the reaching behaviour were scored, with a similar scoring system as described for the humans. The total overall score was the sum of the element scores (possible range: 0 to 11). The sum score was converted to a $0-1$ scale, as described above for the human data.

A full description of the skilled reaching performance scoring in humans and rats is provided in Appendix 2.2.

\section{Fugl-Meyer Upper-Extremity Assessment}

Stroke patients underwent the Fugl-Meyer Upper Extremity (FM-UE) assessment, which is a standard motor performance-based test consisting of 33 tasks performed with the affected 
upper limb. 20,21 The FM-UE assessment focuses on upper limb motor impairment with regard to synergistic motor control. Performance on each task was scored on a 3-point scale, with higher ratings representing better performance (possible range 0 to 66 points). ${ }^{22}$

\section{Action Research Arm Test}

Stroke patients underwent the ARAT, which is a performance test that assesses the ability to perform gross movements and the ability to grasp, move and release objects differing in size, weight and shape. ${ }^{23}$ The test consists of 19 items, rated on 4-point ordinal scale (0-3), with a maximum score of 57 (best performance).

\section{Statistical analysis}

Generalized linear mixed models for human data were used to compare skilled reaching performance on the movement sub-elements at each time-point post-stroke in comparison to the first measurement. This data were treated as interval data as defined by Field and Hole (2003), ${ }^{24}$ and therefore an ordinal logistic regression approach was used. In the analyses we included a random intercept, and time as a fixed effect. Linear mixed models for human and rat data were used to compare reaching performance on the movement elements (rat) and sum scores (human and rat) at each time-point post-stroke.

Linear mixed models were also used to assess temporal changes in patients' relative sum scores (normalized to a percentage of the maximum score of that particular test) for the skilled reaching, ARAT and FM-UE assessments, as well as their mutual relationships. For comparison with the ARAT and FM-UE scoring system, skilled reaching movement scores were reversed for each of the movement elements (i.e. higher scores representing better performance; 0 became 1, 1 became 0).

Pearson's correlation coefficients were calculated to assess the relationship between patients' skilled reaching, ARAT and FM-UE scores at different time-points post-stroke. Significance levels were set at $p=.05$. All statistical analyses were performed using SPSS, version 25.0.

\section{Results}

\section{Subjects}

Twelve patients were included. In ten of these patients, the non-dominant side was affected. Eleven patients were right-handed. Infarcts were subcortical, mixed cortical and subcortical, or in the brainstem, with lesion volumes ranging between 0.4 and $192 \times 10^{3} \mathrm{~mm}^{3}(0.1-26.3 \%$ of the hemispheric volume). Table 2.1 shows the demographic and clinical characteristics. 
From the preclinical study, seventeen rats were included. Three of the seventeen rats had to be euthanized prior to the end of the study (i.e. before day 23) because of welfare issues due to severe weight loss or inner ear infection, resulting in missing data-points. Rats all had stroke on their dominant (for reaching) side. Infarcts were located in the sensorimotor cortex (see Appendix 2.3, Figure S2.4 for representative anatomical MR images) and had a size of $22 \pm 6 \mathrm{~mm}^{3}$ ( $2.9 \pm 0.8 \%$ of the hemispheric volume). Table 2.2 shows the demographic and clinical characteristics.

Table 2.1: Patients' demographic and clinical characteristics at enrollment

\begin{tabular}{ll}
\hline Age, years; mean (SD) & $58.3(10.2)$ \\
Male/Female & $7 / 5$ \\
Handedness & $11 \mathrm{R}, 1 \mathrm{~L}$ \\
Time post-stroke, days; mean (SD) & $14.7(3.6)$ \\
Lesion side & $10 \mathrm{ND}, 2 \mathrm{D}$ \\
Stroke subtype & $8 \mathrm{SC}, 2 \mathrm{M}, 2 \mathrm{~B}$ \\
Lesion volume, $\times 10^{3} \mathrm{~mm}^{3}$; mean (SD) & $21(57)$ \\
FM-UE score; mean (SD) & $18.6(13.1)$ \\
\hline
\end{tabular}

${ }^{1}$ From 11 patients with MRI scan between 6 and 50 weeks post-stroke.

Abbreviations: SD: standard deviation; FM-UE: FM-UE: Fugl-Meyer Upper Extremity; L: left; R: right; D: dominant; ND: non-dominant; SC: subcortical; M: mixed cortical and subcortical; B: brainstem.

\section{Skilled reaching success scores}

The percentages of patients that could successfully perform the skilled reaching task at the different time-points post-stroke are shown in Figure 2.2A. A quarter of the patients could successfully perform skilled reaching at 2-3 weeks post-stroke (early subacute). The success percentage increased over time and was $67 \%$ at $11-15$ weeks after stroke. The success percentage for skilled reaching with the unaffected arm was $100 \%$ at all time-points.

Table 2.2: Rats' demographic and clinical characteristics

\begin{tabular}{ll}
\hline Age, weeks; mean (SD) & $10(0)$ \\
Male/Female & $17 / 0$ \\
Lesion side & $17 \mathrm{D}$ \\
Stroke subtype & $17 \mathrm{C}$ \\
Lesion volume, $\mathrm{mm}^{3}$; mean (SD) & $22(6)$ \\
SR success rate & , \%; mean (SD) \\
\hline
\end{tabular}

${ }^{1}$ From anatomical MRI scan at 17 days post-stroke; ${ }^{2}$ At day 3 post-stroke.

Abbreviations: D: dominant; C: cortical; SD: standard deviation. 
The success percentages at different post-stroke time-points for the rats are shown in Figure 2.2B. The percentage of animals that executed at least one successful reach at the first early subacute time-point ( 3 days post-stroke) was $65 \%$, which subsequently increased. At the final late subacute time-point (23 days post-stroke), $82 \%$ of the rats could successfully execute the skilled reaching task. The success percentage before stroke was $100 \%$. The conventionally calculated success rate in rats, i.e. the percentage of successfully obtained pellets with respect to the number of trials, was $38 \pm 17 \%$ before stroke, dropped to 17 $\pm 16 \%$ at 3 days after stroke, and partially recovered to $25 \pm 20 \%$ at day 23 post-stroke (Appendix 2.3, Figure S2.5).

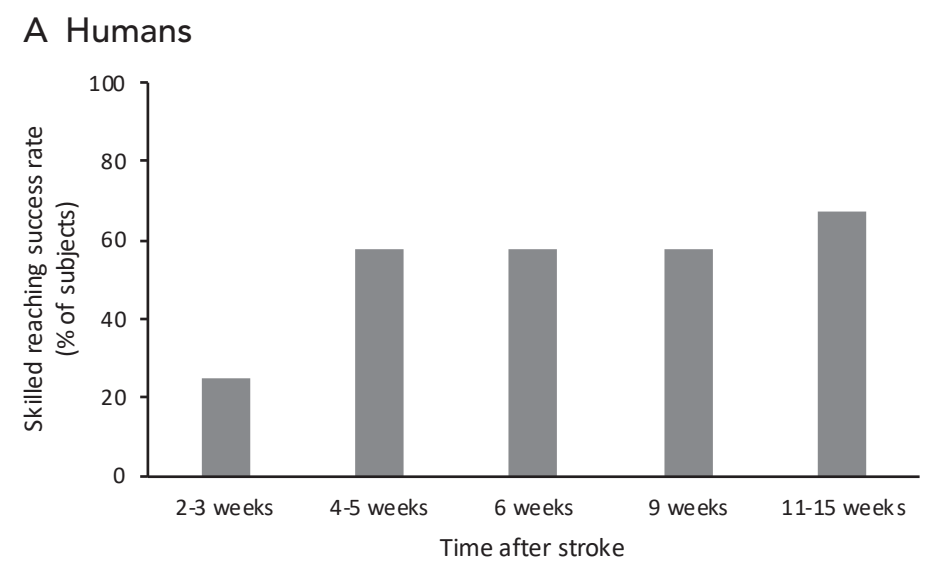

\section{B Rats}

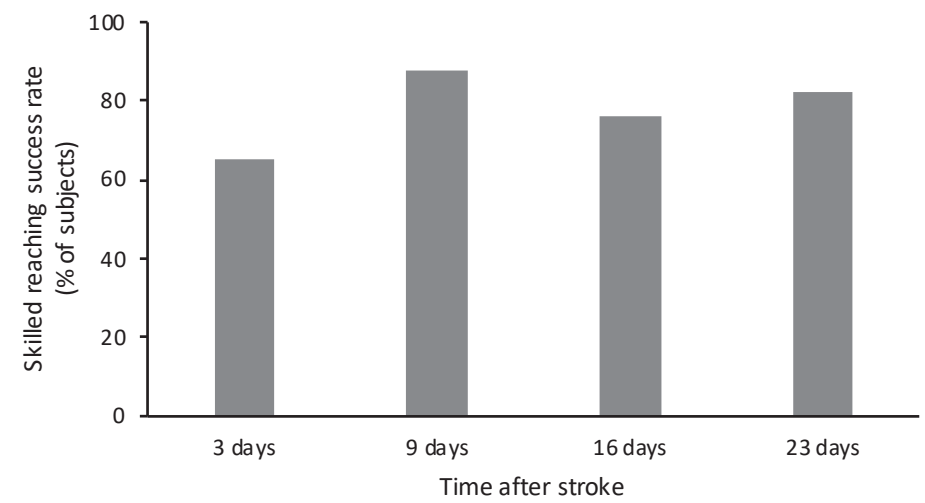

Figure 2.2: Success rate of skilled reaching performance (\% of subjects) at different time-points after stroke in human patients (top) and rats (bottom).

Humans had a success rate of $100 \%$ with the unaffected arm at each time-point. Rats had a success rate of $100 \%$ pre-stroke. 


\section{Movement (sub)element scores}

Figure 2.3 shows the patient and rat scores for the individual movement (sub)elements during execution of the skilled reaching task at the different post-stroke time-points. At all post-stroke time-points, the movement element orient was unaffected in patients as well as rats, reflected by a score of 0 for the orient (sub)element(s). All other movement elements were affected (i.e. incomplete or absent) as a result of the stroke, although scores were generally higher - reflective of a higher degree of deficiency - in patients than in rats. At the second time-point (4-5 weeks after stroke in patients; 9 days after stroke in rats) there were significant improvements for most movement elements in comparison to the first measurement $(p<.05)$, except for the movement sub-elements advance $C$, pronation $C$, grasp $B$ and release $B$ in patients $(p>.05)$. In rats, there were significant improvements for the movement elements supination (I and II) and release at the second time-point $(p<.05)$, and additional improvements for the movement elements supination I and release were measured at subsequent time-points.

From week six towards the last time-point in patients, most movement elements, including sub-elements grasp $B$ and advance $C$, showed significant improvement $(p<.05)$. Movement sub-elements pronation $C$ and release $B$, however, showed no significant improvement at weeks six and nine.

At the final time-point (11-15 weeks after stroke in patients; 23 days after stroke in rats) all movement sub-elements in patients, except for release $B$, were significantly improved compared to the first time-point $(p<.05)$. In contrast, in rats only the movement elements digits open, supination I and II, and release were significantly improved at the final timepoint as compared to the first time-point $(p<.05)$.

Recovery of skilled reaching performance was also expressed by the change in the patients' and rats' skilled reaching overall sum score, which significantly improved between the first time-point, and all subsequent time-points $(p<.05)$.

Relationship between skilled reaching and clinical outcome measures in stroke patients Figure 2.4 shows the time-course of normalized skilled reaching, FM-UE and ARAT sum scores for the stroke patients. For all three measures, the scores at the 6, 9, and 11-15 weeks were significantly improved in comparison to the scores at 2-3 weeks post-stroke (skilled reaching: $\beta=11.53, \mathrm{SE}=2.18, p=.000$ : ARAT: $\beta=14.82, \mathrm{SE}=3.09, p=.000$; and FM-UE: $\beta=11.88, S E=2.11, p=.000$ ) (FM-UA assessment was not performed at 4-5 weeks post-stroke, so we left out this time-point for comparisons). The normalized skilled reaching and FM-UE sum scores were highly similar at the individual time-points $(\beta=1.75$, $S E=6.12, p=.775)$, which was further emphasized by similar temporal recovery patterns 
A Humans

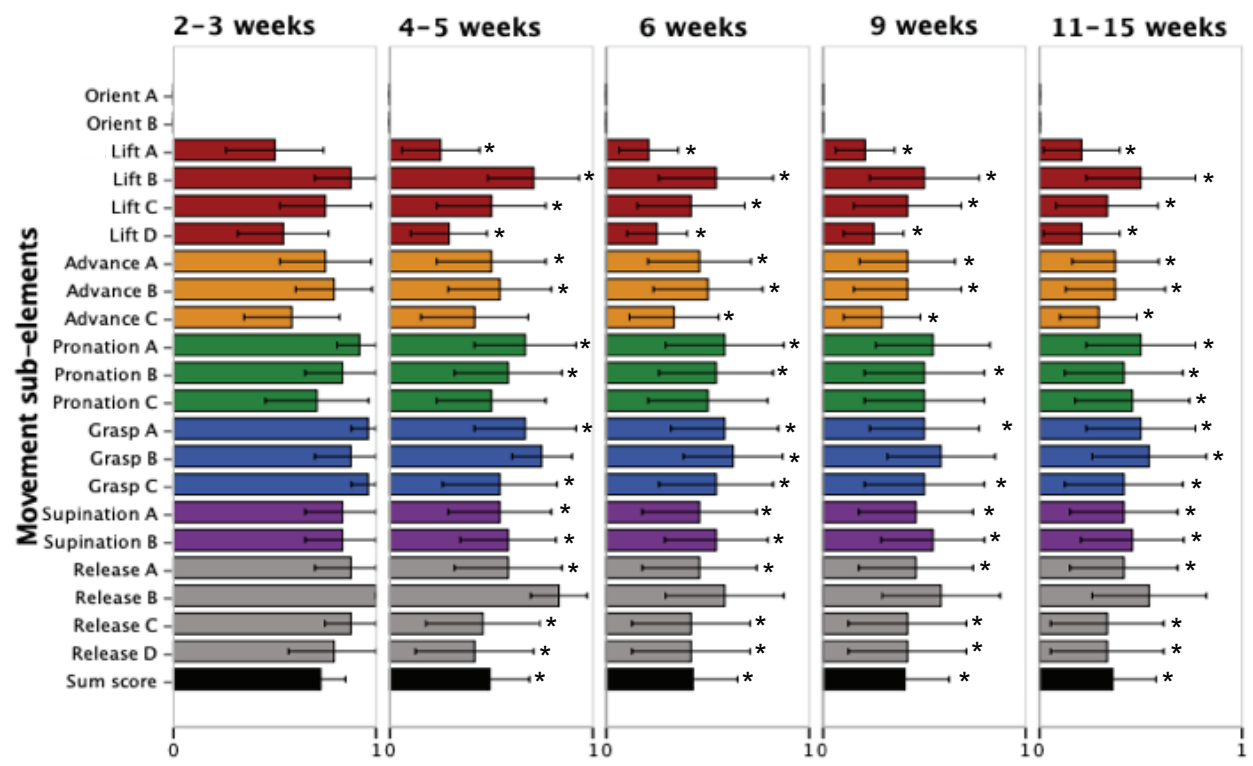

B Rats

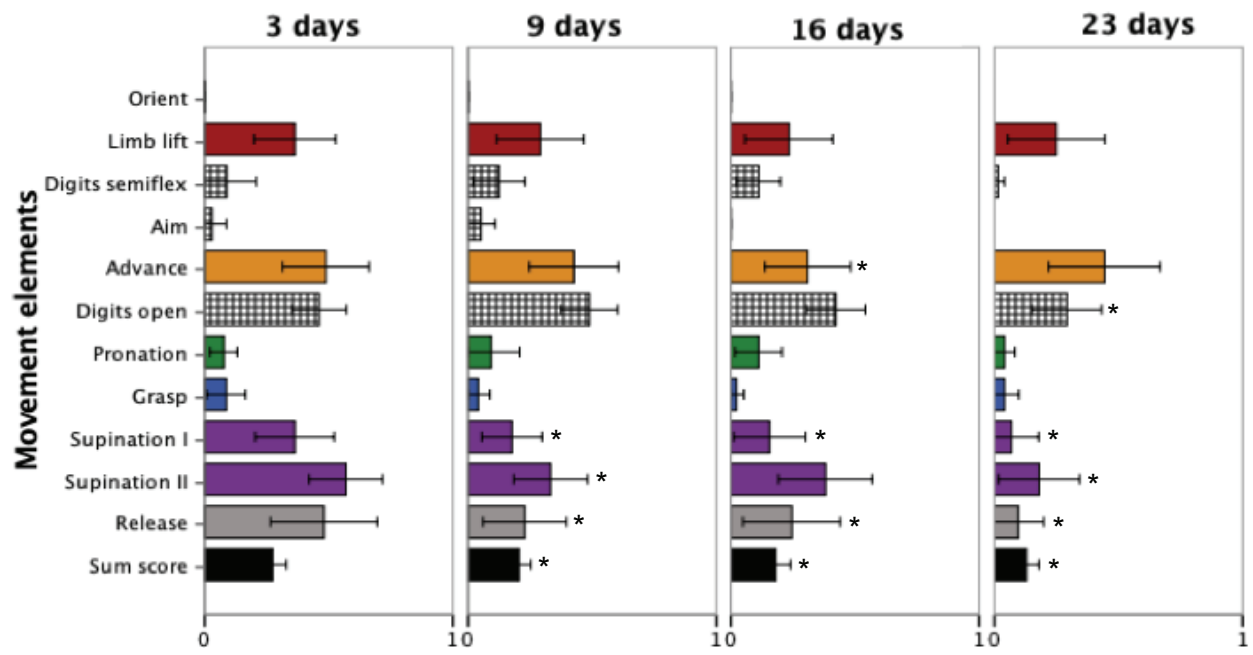

Figure 2.3: Movement (sub)element scores for patients (A) and rats (B) at different time-points after stroke.

Each bar represents the mean \pm SD; Non-colored elements, displayed as black-white blocked bars, are rat-specific elements; 0 : movement is present/normal, 0.5 : movement is present but incomplete, 1: no movement; *Significant difference $(p<.05)$. 


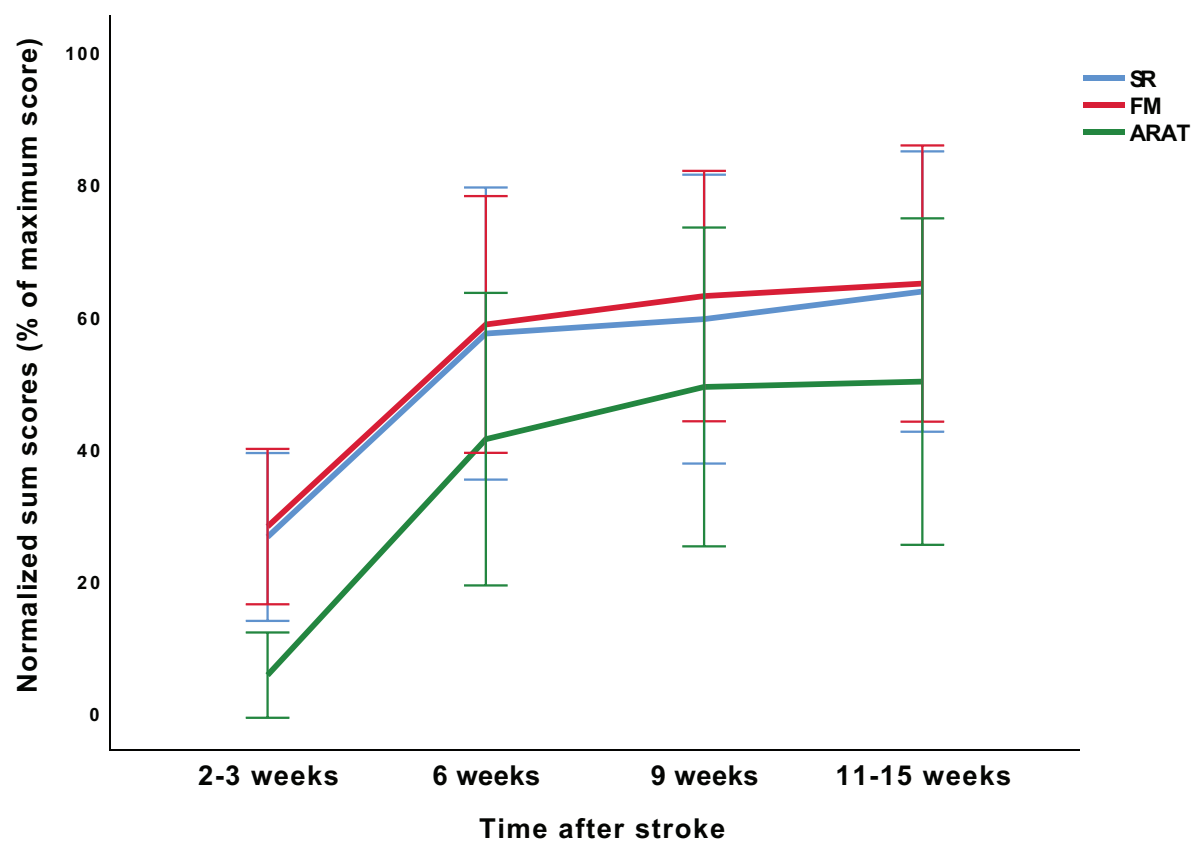

Figure 2.4: Relative sum scores (normalized to the maximum score of that particular test) from skilled reaching (SR), ARAT and FM-UE assessments of stroke patients over time.

Skilled reaching scores were reversed (i.e. higher scores representing better performance) for comparison with the FM-UE and ARAT scores. Error bars represent standard error of the mean.

$(\beta=.10, S E=3.27, p=.975)$. The normalized skilled reaching and ARAT sum scores differed significantly across separate time-points post-stroke $(\beta=-19.11, S E=6.13, p=.002)$. However, the temporal patterns of the normalized skilled reaching and ARAT sum scores were not significantly different $(\beta=2.46, S E=3.31, p=.459)$.

Table 2.3 shows that skilled reaching, ARAT and FM-UE sum scores were strongly correlated at all included time-points post-stroke $(p<.05)$.

Table 2.3: Pearson's correlations between patients' skilled reaching, ARAT and FM-UE sum scores at different time-points after stroke

\begin{tabular}{lllll}
\hline & \multicolumn{4}{c}{ Skilled reaching } \\
\cline { 2 - 5 } & $2-3$ weeks & 6 weeks & 9 weeks & $11-15$ weeks \\
\hline ARAT & $.647^{\star}$ & $.888^{\star}$ & $.871^{\star}$ & $.963^{\star}$ \\
FM-UE & $.717^{\star}$ & $.917^{\star}$ & $.911^{\star}$ & $.966^{\star}$ \\
\hline
\end{tabular}

${ }^{*} p<.05$. Abbreviations: ARAT: Action Research Arm Test; FM-UE: Fugl-Meyer Upper Extremity. 


\section{Discussion}

Skilled reaching has been proposed as one of the most potent translational behavioral tests for studying post-stroke recovery in rodents. ${ }^{9}$ Gradual improvement in skilled reaching performance after stroke has been previously reported for both humans ${ }^{25}$ and rodents. ${ }^{26}$ In the present study we compared skilled reaching characteristics between rats and humans recovering from stroke, and we assessed the relation between skilled reaching performance and clinical outcome measures. Our study shows that skilled reaching performance follows a very comparable temporal pattern in humans and rodents during the subacute stages after stroke. Functional impairment was characterized by muscle flaccidity at the first measurement post-stroke, prohibiting lift and advance movements. Subsequently, skilled reaching performance improved, but with a delay in motor recovery for distal muscles in the lower arm and hand (i.e. pronation, grasp and release). Patients' skilled reaching scores showed strong correlations with clinical outcome measures (ARAT and FM-UE) at different time-points during the first three months post-stroke. The normalized skilled reaching overall sum scores matched with the normalized FM-UE sum scores, but were higher than the sum scores of an arm-specific measure of activity limitation (ARAT).

Similarities and differences in post-stroke recovery of skilled reaching performance between humans and rats

It is known that the time-course of stroke recovery in rodent models is more rapid than in human patients. ${ }^{27-30}$ Therefore, we chose to compare the first three months post-stroke in human stroke patients to the first 3-4 weeks post-stroke in rats, which is the typical period for reaching a plateau stage of motor recovery in these species, respectively. ${ }^{15}$

Success rate is the most commonly used outcome parameter to quantify functional deficits from a skilled reaching test. In rodent studies, success rates are typically calculated as the number of successful reaches divided by the number of trials, multiplied by $100 \% .^{12}$ In the current study, we also applied an adapted success percentage score, defined as the number of rats that could completely execute at least one successful reach, divided by the total number of rats, and multiplied by $100 \%$, which was identical to the scoring system for the stroke patients. Success percentages for both humans and rats showed post-stroke impairment in skilled reaching. The scores for human patients reflected more severe initial deficits, but a larger degree of subsequent recovery, as compared to the post-stroke rats. This may be explained by differences in stroke severity and phase between the stroke patients and our rat stroke model, as further outlined below. In addition, humans had to grasp a smaller food item relative to hand size in comparison to rats, which may have made the task more challenging. While rats apply similar grasp patterns (whole paw grasp) for 
small and large food items, humans use pincer grasp for small items and precision grasp for larger items. ${ }^{12}$ Of note, variation in baseline skilled reaching abilities, which for example has been observed between different rat strains, may also contribute to differences in recovery patterns. ${ }^{18}$

Previous studies in rat models of stroke have shown that skilled reaching success rates may not expose remaining motor deficits, ${ }^{26,31-33}$ which may be better assessed by analysis of the skilled reaching movement elements. The movements that articulate skilled reaching displayed similar recovery patterns between humans and rats. This finding emphasizes the translational value of skilled reaching testing for preclinical and clinical research on stroke recovery. At the early sub-acute stage post-stroke, muscle flaccidity could be observed in both species, which contributes to complications in the voluntary execution of movement elements, such as lift and advance. Body-postural compensation, with stronger reliance on proximal muscles, was observed, and related functions, e.g. lift, were less affected or showed rapid recovery. In human stroke patients, functional recovery of the distal muscles in the lower arm, responsible for pronation, grasp and release, showed no (release B) or delayed improvement (pronation $C$ and grasp $B$ ) in the late subacute phase post-stroke. Correspondingly, in rats proximal movement elements (i.e. limb lift, digits semi-flex, and aim) were less affected at the first sub-acute measurement, while distal movement elements (i.e. supination, release) and particularly digit motor control (i.e. digits open) still improved towards late sub-acute stages. These findings are in line with results from other studies that described recovery of proximal joint movements preceding recovery of distal movements, suggesting differences in neural substrates for paretic upper limb recovery. ${ }^{22,34,35}$ In our study, the relative infarct size in rats was within the range of relative infarct sizes in stroke patients. Hemispheric infarct volume was about $3 \%$ in rats and ranged between 0.1 and $26 \%$ in humans. However, in contrast to the variety in lesion location in patients, the stroke lesion in rats was confined to the sensorimotor cortex, leaving large part of the sensorimotor system intact. This may have facilitated the progression of recovery. Nevertheless, several movement elements (e.g., partial rotation) remained impaired at the final measurement post-stroke, consistent with an earlier study in the same rodent stroke model. ${ }^{26}$ This contrasts with other commonly used behavioral measures of sensorimotor function (e.g. fore- or hindlimb placing ability) that often show complete recovery within 2-4 weeks after stroke, which emphasizes the sensitivity of the skilled reaching test. ${ }^{36}$

The human stroke patients in our study, who had varying infarct locations, also showed significant persistence of upper limb impairment in the first three months post-stroke, which is in accordance with previous studies. ${ }^{37,38}$ The persistence of impairment in human and rat skilled reaching movements suggests that some recovery could have been achieved through use of compensatory strategies. 
Relation between skilled reaching performance and clinical outcome measures

Our study showed strong positive correlations between the normalized sum scores of skilled reaching and clinical outcome measures from the ARAT and FM-UE assessment at each of the time-points during the first three months post-stroke. The three tests share measurement of reach and lift components, and a lower level of impairment on these elements will be reflected in better (functional) performance across a broad range of movements. ${ }^{39,40}$ In addition, the design of the motor section of the FM-UE assessment is based on synergies, i.e. systematic coupling across different joints or a fixed pattern of coactivation of muscles, of which disruptions result in reaching deficits. ${ }^{39,41}$ Importantly, because there are no equivalents of the ARAT or FM-UE assessment for rodents, the correlation between skilled reaching performance and these clinical outcome measures highlight the significance of skilled reaching assessment in the alignment of preclinical and clinical stroke research.

Consistent with previous research, skilled reaching scores and clinical outcome scores improved significantly over the three-month period post-stroke. ${ }^{11}$ The largest improvements were seen in the first 5-6 weeks post-stroke. In general, spontaneous neurological recovery progresses fastest in the first months post-stroke, after which recovery levels off and reaches a plateau after 3 to 6 months. ${ }^{42,43}$ Interestingly, the ARAT total scores were significantly different from the skilled reaching total scores. The ARAT, which assesses activity limitation, presented lower normalized baseline scores compared to the skilled reaching and FM-UE tests. Features of the measures, such as the representation of specific parts of the upper limb within the individual measures, may underlie the differences in scores. In the ARAT 16\% of the total score is obtained from assessment of gross arm movements (i.e. placement of hand behind head, on top of head, and to the mouth), while the majority of the score is based on movements that require some form of (finer) hand and digit motor control. Fine motor control involves motor coordination, speed of movement, and force scaling. ${ }^{44}$ In contrast, in skilled reaching testing the first relatively gross movement elements (orient, lift, advance, and pronation) make up $57 \%$ of the total score, whereas grasp, which requires fine hand and digit motor control, constitute $43 \%$ of the total score. ${ }^{18,45}$ Our findings demonstrate that motor recovery follows a proximal-distal principle, which is in agreement with Twitchell and Brunnstrom's concept of sequential return of motor function in the hemiplegic stroke patient, where hand and digit motor control recover at later stages. ${ }^{22}$ The underrepresentation of distal fine motor function may explain the higher scores on the FM-UE and skilled reaching tests as compared to the ARAT scores in the sub-acute period post-stroke.

One of the strengths of the skilled reaching task is its ability to allow for distinction between compensation and recovery. ${ }^{15}$ This is in contrast with the ARAT, which is a performancebased measure focused on task accomplishment, with little regard for how the task is accomplished. The ARAT therefore precludes distinguishment between true motor recovery 
and compensatory movement strategies. ${ }^{6}$ The FM-UE assessment is largely immune to compensation, and can be valuable for monitoring true recovery of motor functions. However, the FM-UE test is infrequently used today in clinical practice, as it is a timeconsuming measure to administer. ${ }^{39}$

In the past 40 years, clinical priority shifted away from impairment-oriented training towards training of activities of daily living with functional tasks, since relearning normal patterns of movement did not inevitably generalize to activities of daily living. ${ }^{39,46}$ Our current findings suggest that restoration of motor function is still feasible up to at least 3 months post-stroke. This implies that impairment-oriented training could still be relevant in motor rehabilitation, and underscores the significance of incorporating measures at the impairment level to distinguish between true motor recovery and compensation after stroke in (pre)clinical research and in clinical practice.

\section{Limitations}

Although our study enabled direct translation of post-stroke skilled reaching performance assessments between rats and humans after stroke, there were some limitations. First, we could not consider the impact of handedness on outcome measures, since only two patients had an affected dominant upper limb. While rats were trained to use their dominant/ preferred forelimb to reach for the target, humans were instructed to use their affected limb, which was not always the dominant one. Second, we did not assess other factors, such as vision, olfaction, fatigue or self-efficacy, which may have contributed to reach-to-eat performance in rats and patients. Third, no kinematic assessment was performed, which could have improved the sensitivity to capture subtle movement qualities and compensatory motions. ${ }^{47}$ Fourth, patients and rats in our study were subjects in ongoing clinical and preclinical trials, respectively, in which the effect of rTMS on motor recovery is investigated. The intervention may have impacted the recovery profiles.

\section{Conclusions}

This study shows that skilled reaching performance improves significantly over time in humans and rats recovering from stroke. Both species showed muscle flaccidity in the early sub-acute phase, early recovery of proximal movements, and a delayed motor recovery of distal muscles in the lower arm/forelimb. The recovery of skilled reaching performance in human stroke patients strongly resembled the recovery patterns of commonly used clinical outcome measures from the ARAT and FM-UE assessment, which underlines the translational significance of the skilled reaching task in preclinical research. Furthermore, skilled reaching assessment can serve as a complementary tool to distinguish between recovery and compensation in clinical care. 


\section{Acknowledgements}

This work was supported by the Netherlands Organization for Scientific Research [VICl 016.130.662].

\section{References}

1. Lawrence, E. S. et al. Estimates of the prevalence of acute stroke impairments and disability in a multiethnic population. Stroke 32, 1279-1284 (2001).

2. Lee, K. B. et al. Six-month functional recovery of stroke patients: A multi-time-point study. Int. J. Rehabil. Res. 38, 173-180 (2015).

3. Nichols-Larsen, D. S., Clark, P. C., Zeringue, A., Greenspan, A. \& Blanton, S. Factors influencing stroke survivors' quality of life during subacute recovery. Stroke 36, 1480-1484 (2005).

4. Foroud, A. \& Whishaw, I. Q. Changes in the kinematic structure and non-kinematic features of movements during skilled reaching after stroke: A Laban Movement Analysis in two case studies. J. Neurosci. Methods 158, 137-149 (2006).

5. Taub, E., Uswatte, G. \& Mark, V. W. The functional significance of cortical reorganization and the parallel development of Cl therapy. Front. Hum. Neurosci. 8, 396 (2014).

6. Levin, M. F., Kleim, J. A. \& Wolf, S. L. What Do Motor Recovery and Compensation Mean in Patients Following Stroke? Neurorehabil. Neural Repair 23, 313-319 (2008).

7. Jones, T. Motor compensation and its effects on neural reorganization after stroke. Nat. Rev. Neurosci. 18, 276-278 (2017).

8. Corbett, D. et al. Enhancing the alignment of the preclinical and clinical stroke recovery research pipeline: Consensus-based core recommendations from the Stroke Recovery and Rehabilitation Roundtable translational working group. Int. J. Stroke 12, 462-471 (2017).

9. Klein, A., Sacrey, L. A. R., Whishaw, I. Q. \& Dunnett, S. B. The use of rodent skilled reaching as a translational model for investigating brain damage and disease. Neurosci. Biobehav. Rev. 36, 1030-1042 (2012).

10. Whishaw, I. Q. \& Pellis, S. M. The structure of skilled forelimb reaching in the rat: A proximally driven movement with a single distal rotatory component. Behav. Brain Res. 41, 49-59 (1990).

11. Sacrey, L. A. R., Alaverdashvili, M. \& Whishaw, I. Q. Similar hand shaping in reaching-for-food (skilled reaching) in rats and humans provides evidence of homology in release, collection, and manipulation movements. Behav. Brain Res. 204, 153-161 (2009).

12. Whishaw, I. Q., Pellis, S. M. \& Gorny, B. P. Skilled reaching in rats and humans: evidence for parallel development or homology. Behav. Brain Res. 47, 59-70 (1992).

13. Alaverdashvili, M. \& Whishaw, I. Q. A behavioral method for identifying recovery and compensation: Hand use in a preclinical stroke model using the single pellet reaching task. Neurosci. Biobehav. Rev. 37, 950-967 (2013).

14. Balkaya, M. \& Cho, S. Optimizing functional outcome endpoints for stroke recovery studies. J. Cereb. Blood Flow Metab. 39, 2323-2342 (2019).

15. Krakauer, J. W., Carmichael, S. T., Corbett, D. \& Wittenberg, G. F. Getting neurorehabilitation right: what can be learned from animal models? Neurorehabil. Neural Repair 26, 923-931 (2012).

16. van Lieshout, E. C. C., Visser-Meily, J. M. A., Neggers, S. F. W., van der Worp, H. B. \& Dijkhuizen, R. M. Brain stimulation for arm recovery after stroke (B-STARS): protocol for a randomised controlled trial in subacute stroke patients. BMJ Open 7, e016566 (2017). 
17. McDonald, M. W., Hayward, K. S., Rosbergen, I. C. M., Jeffers, M. S. \& Corbett, D. Is environmental enrichment ready for clinical application in human post-stroke rehabilitation? Front. Behav. Neurosci. 12, 135 (2018).

18. Whishaw, I. Q., Gorny, B., Foroud, A. \& Kleim, J. A. Long-Evans and Sprague-Dawley rats have similar skilled reaching success and limb representations in motor cortex but different movements: Some cautionary insights into the selection of rat strains for neurobiological motor research. Behav. Brain Res. 145, 221-232 (2003).

19. Eshkol, N. \& Wachmann, A. Movement Notation. (Weidenfeld and Nicolson, 1958).

20. Duncan, P. W., Propst, M. \& Nelson, S. G. Reliability of the Fugl-Meyer assessment of sensorimotor recovery following cerebrovascular accident. Phys. Ther. 63, 1606-1610 (1983).

21. Fugl Meyer, A. R., Jaasko, L. \& Leyman, I. The post stroke hemiplegic patient. I. A method for evaluation of physical performance. Scand. J. Rehabil. Med. 7, 13-31 (1975).

22. Gladstone, D. J., Danells, C. J. \& Black, S. E. The fugl-meyer assessment of motor recovery after stroke: A critical review of its measurement properties. Neurorehabil. Neural Repair 16, 232-240 (2002).

23. van der Lee, J. H., Roorda, L. D., Beckerman, H., Lankhorst, G. J. \& Bouter, L. M. Improving the Action Research Arm test: a unidimensional hierarchical scale. Clin. Rehabil. 16, 646-653 (2002).

24. Field, A. \& Hole, G. How to design and report experiments. (SAGE Publications Sage UK: London, England, 2003).

25. Foroud, A. \& Whishaw, I. Q. Reaching-to-Eat in Humans Post-Stroke: Fluctuating Components Within a Constant Pattern. Behav. Neurosci. 124, 851-867 (2010).

26. Moon, S. K., Alaverdashvili, M., Cross, A. R. \& Whishaw, I. Q. Both compensation and recovery of skilled reaching following small photothrombotic stroke to motor cortex in the rat. Exp. Neurol. 218, 145-153 (2009).

27. Langhorne, P., Bernhardt, J. \& Kwakkel, G. Stroke rehabilitation. Lancet 377, 1693-1702 (2011).

28. Kwakkel, G. et al. Effects of unilateral upper limb training in two distinct prognostic groups early after stroke. The EXPLICIT-stroke randomized clinical trial. Neurorehabil. Neural Repair 30, 804-816 (2016).

29. Winters, C., Van Wegen, E. E. H., Daffertshofer, A. \& Kwakkel, G. Generalizability of the Proportional Recovery Model for the Upper Extremity After an Ischemic Stroke. Neurorehabil. Neural Repair 29, 614-622 (2015).

30. Sengupta, P. The laboratory rat: Relating its age with human's. Int. J. Prev. Med. 4, 624-630 (2013).

31. Alaverdashvili, M., Foroud, A., Lim, D. H. \& Whishaw, I. O. 'Learned baduse' limits recovery of skilled reaching for food after forelimb motor cortex stroke in rats: A new analysis of the effect of gestures on success. Behav. Brain Res. 188, 281-290 (2008).

32. Metz, G. A., Antonow-Schlorke, I. \& Witte, O. W. Motor improvements after focal cortical ischemia in adult rats are mediated by compensatory mechanisms. Behav. Brain Res. 162, 71-82 (2005).

33. Gharbawie, O. A. \& Whishaw, I. Q. Parallel stages of learning and recovery of skilled reaching after motor cortex stroke: 'Oppositions' organize normal and compensatory movements. Behav. Brain Res. 175, 249-262 (2006).

34. Luke, C., Dodd, K. J. \& Brock, K. Outcomes of the Bobath concept on upper limb recovery following stroke. Clin. Rehabil. 18, 888-898 (2004).

35. Schambra, H. M. et al. Differential Poststroke Motor Recovery in an Arm Versus Hand Muscle in the Absence of Motor Evoked Potentials. Neurorehabil. Neural Repair 33, 568-580 (2019).

36. Madinier, A., Quattromani, M. J., Sjölund, C., Ruscher, K. \& Wieloch, T. Enriched housing enhances recovery of limb placement ability and reduces aggrecan-containing perineuronal nets in the rat somatosensory cortex after experimental stroke. PLoS One 9, e93121 (2014). 
37. Kwakkel, G., Kollen, B. J., Van der Grond, J. V. \& Prevo, A. J. H. Probability of regaining dexterity in the flaccid upper limb: Impact of severity of paresis and time since onset in acute stroke. Stroke 34, 2181-2186 (2003).

38. Brunner, I. C., Skouen, J. S. \& Strand, L. I. Recovery of upper extremity motor function post stroke with regard to eligibility for constraint-induced movement therapy. Top. Stroke Rehabil. 18, 248-257 (2011).

39. Krakauer, J. W. \& Carmichael, S. T. Broken Movement. Broken Movement (2019). doi:10.7551/ mitpress/9310.001.0001

40. Nijland, R. H. M., Van Wegen, E. E. H., Harmeling-Van Der Wel, B. C. \& Kwakkel, G. Presence of finger extension and shoulder abduction within 72 hours after stroke predicts functional recovery: Early prediction of functional outcome after stroke: The EPOS cohort study. Stroke 41, 745-750 (2010).

41. Balbinot, G. et al. Post-stroke kinematic analysis in rats reveals similar reaching abnormalities as humans. Sci. Rep. 8, 1-13 (2018).

42. Krakauer, J. W. \& Marshall, R. S. The proportional recovery rule for stroke revisited. Ann. Neurol. 78, 845-847 (2015).

43. Schaechter, J. D. Motor rehabilitation and brain plasticity after hemiparetic stroke. Prog. Neurobiol. 73, 61-72 (2004).

44. Allgöwer, K. \& Hermsdörfer, J. Fine motor skills predict performance in the Jebsen Taylor Hand Function Test after stroke. Clin. Neurophysiol. 128, 1858-1871 (2017).

45. Lang, C. E., Wagner, J. M., Dromerick, A. W. \& Edwards, D. F. Measurement of Upper-Extremity Function Early After Stroke: Properties of the Action Research Arm Test. Arch. Phys. Med. Rehabil. 87, 1605-1610 (2006).

46. Carr, J. H. Movement science: Foundations for physical therapy in rehabilitation. (Aspen Publishers, 1987).

47. Bernhardt, J. et al. Agreed definitions and a shared vision for new standards in stroke recovery research : The Stroke Recovery and Rehabilitation Roundtable taskforce. Int. J. Stroke 12, 444-450 (2017). 


\section{Appendix 2.1}

\section{Housing (rats)}

Rats were housed per 2 or 3 in standard cages $\left(30 \times 40 \times 20 \mathrm{~cm}^{3}\right)$, during the skilled pellet reaching training phase, up until day 2 after stroke (see Figure S2.1). The standard cages were equipped with bedding material, cage enrichment (orange rectangular Perplex tube) and nesting material such as tissue paper.

Three days after stroke induction, the animals were moved to an enriched environment cage that was larger $\left(70 \times 60 \times 50 \mathrm{~cm}^{3}\right)$, contained multiple replaceable toys, platforms and ladders (changed twice per week), and housed 4-5 rats. This enriched environment represented a clinical rehabilitation setting.

\section{Skilled pellet reaching (rats)}

\section{Food restriction}

Once the animals ( 6 weeks old) arrived at the animal facility, they were allowed to acclimatize to their new environment for five days under normal housing conditions and with ad libitum access to food and water (see timeline in Figure S2.1). Following acclimatization, animals were weighed and placed on food restriction to maintain 90-95\% of their initial body weight. The animals, housed in pairs, received 26-30 grams of chow per day and they received water ad libitum. The food restriction functioned as a motivator for the animals to learn and to participate in the skilled pellet reaching task.

During the first week of food restriction, the animals also received a few sugar pellets (45 mg sucrose, unflavored; Dustless Precision Pellets, Bio-Serve ${ }^{\circledR}$ ) with their chow to get accustomed to their taste. Following this first week, the animals started with the pre-training phase. The body weights of the animals were closely monitored throughout the entire experiment.

\section{Pre-training}

During pre-training, the goal was to stimulate the grasping of sugar pellets through the slot and to determine the dominant/preferred forelimb of each animal. The rats were placed in a Plexiglas box with multiple vertical slots in the front panel of the box (Figure S2.2). A plastic cup, filled with sugar pellets to the brim, was placed in front of the vertical slots. The animals were allowed to grasp as many sugar pellets as they could within a 30 minute period. During this period, the grasping behavior of the animals was observed in order to determine whether they had a preferred grasping-paw. After three consecutive days of pretraining a preferred forelimb could be identified and this was regarded as the dominant paw. 


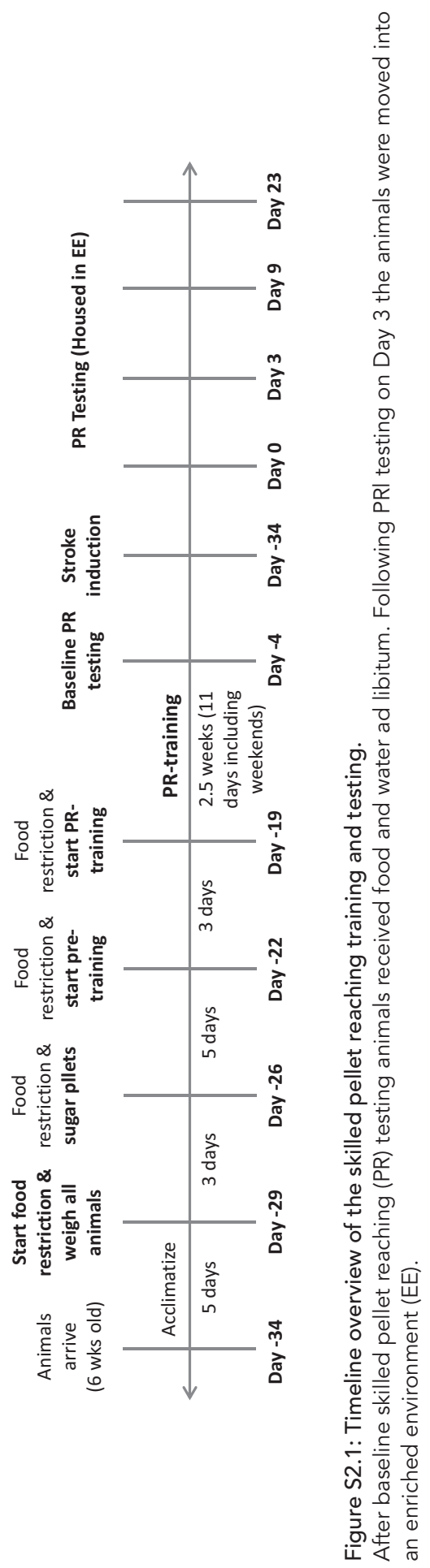




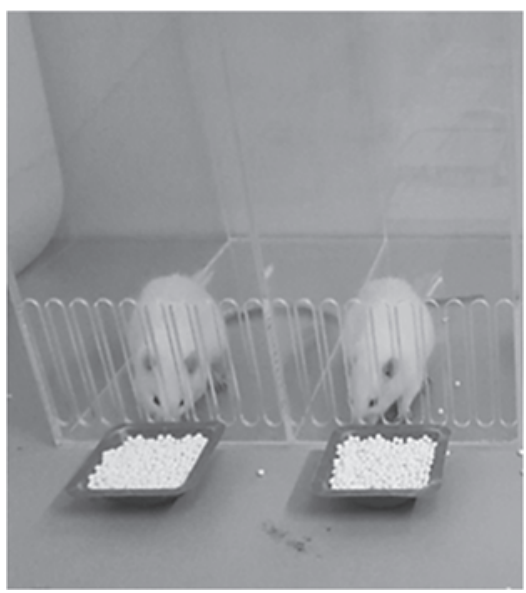

Figure S2.2: Skilled pellet reaching pre-training box.

This Plexiglas training box allows for the simultaneous training of multiple animals at the same time.

\section{Training}

Following the pre-training step, each individual rat was trained to perform the skilled pellet reaching task in one of two custom-built, clear, Plexiglas boxes (Figure S2.3). Both training boxes allowed the same training (no difference in training effect was observed between the two boxes (data not shown)), however one box was specifically designed for unobstructed high-speed recording of the grasping movement from the lateral aspect of the box (Figure S2.3A). In each of the training boxes, there were one or two vertical slots in the front panel of the box. At the bottom of each vertical slot, there was a shelf with an indentation that allowed pellet placement slightly off-center to the opening of the slot. The off-center positioning of the pellet was necessary to force the rats to use their 'dominant' forelimb to grasp the sugar pellet. The slot ipsilateral to the side of the preferred paw was used to train the animal (Figure S2.3A; $n=5$ ). In the other skilled pellet reaching box (Figure S2.3B; $n=12$ ), the sugar pellet was positioned on the indent contralateral to the preferred paw to train the animal.

Skilled pellet reaching training was performed for a maximum period of 15 minutes per day, Monday through Friday, for 2.5 weeks in one of the respective training boxes.. The animals were trained to approach the shelf at the front of the box, reach for a pellet, and then turn around to walk to the rear of the box. If the rat succeeded to grasp the pellet from the shelf, it was considered a successful trial. Before a new trial could start, the animal first had to walk to the rear end of the box again before a new pellet could be obtained. If the rat knocked the pellet off of its original position, the pellet was removed from the shelf and it was regarded as a failed attempt. 
A)

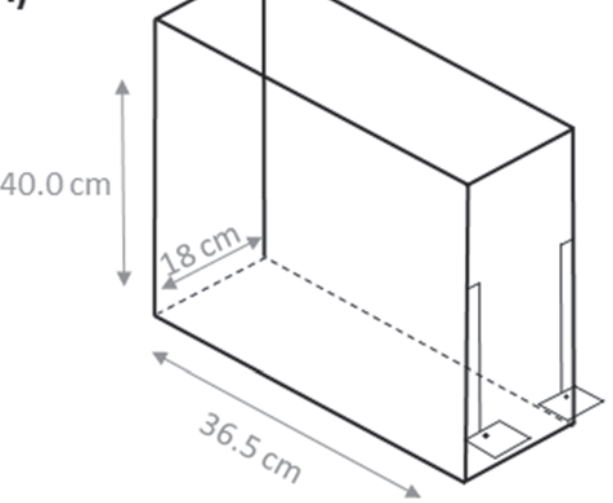

B)

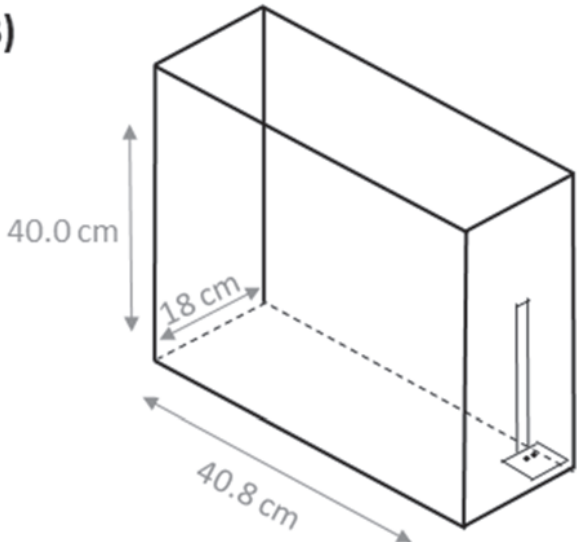

Figure S2.3: Skilled pellet reaching boxes.

(A) Training box with two vertical slots of $1 \mathrm{~cm}$ wide, which extended $3 \mathrm{~cm}$ above the floor, located at the left and right end of the front side. At the bottom of the slots, there was a $3 \mathrm{~cm}$ wide shelf with an indentation that allowed pellet placement slightly lateral to the opening of the slot. To make sure the rat approached the correct slot, the other slot was blocked with a metal object.

(B) Training box with a single vertical slot of $1 \mathrm{~cm}$ wide in the center of the front side, which extended 3 $\mathrm{cm}$ above the floor. At the bottom of the vertical slot there was a $4.5 \mathrm{~cm}$ wide shelf with two indentations that allowed for the placement and grasping of a sugar pellet from the indent contralateral to the preferred paw.

\section{Data acquisition}

On the behavioral testing days the skilled pellet reaching task was recorded from the frontal plane using a Panasonic HC V520 camera (50 fps). These recordings were used to for the quantitative and qualitative pellet reaching analyses. During the testing sessions light was provided from two light sources in the direction that was filmed.

\section{Photothrombotic stroke induction (rats)}

A photothrombotic stroke was induced in the hemisphere contralateral to the preferred forelimb. ${ }^{1,2}$ Pre-operatively, all rats received $5 \mathrm{mg} / \mathrm{kg}$ carprofen subcutaneously to minimize pain during and after surgery. Rats were anesthetized with isoflurane (5\% induction, $2 \%$ maintenance in air/ $\left.\mathrm{O}_{2}(4 / 1)\right)$, intubated for mechanical ventilation, and placed in a stereotaxic frame. A feedback-controlled heating pad ensured that the body temperature of the animals was maintained at $37.0 \pm 1.0^{\circ} \mathrm{C}$. After shaving of the head, the skull was disinfected with $70 \%$ alcohol and a midline incision was made along the scalp. Xylocaine (Lidocaine spray $100 \mathrm{mg} / \mathrm{ml}$, AstraZeneca B.V. Louis, Zoetermeer) was applied in the incision for local analgesia. Using a scalpel, the periosteum was scraped away and the skull was dried with sterile cotton swabs. The coordinates of bregma were determined using a stereotact and 
the sensorimotor cortex was identified as a rectangular section 1.5 to $4.5 \mathrm{~mm}$ lateral to bregma, and 4.0 to $-4.0 \mathrm{~mm}$ anterior-posterior to bregma. Black tape was used to mask the skull outside of the region of interest. Next, Rose Bengal $(25$ mg/ml, Sigma Aldrich/Merck, The Netherlands), a photosensitive dye, was injected intravenously via the vena saphena, and the brain was illuminated through the skull for 20 minutes, using a cold light source with a green fluorescent filter (Schott KL 1500 LCD, Germany). Following illumination, the scalp was sutured and the animal was placed in a heated cage to recover.

\section{Repetitive transcranial magnetic stimulation (rats)}

From days six to sixteen post-stroke, the rats received repetitive transcranial magnetic stimulation (rTMS) treatment. This excluded weekend days, resulting in a total of nine treatment days. The stimulation protocols were executed with a $25-\mathrm{mm}$ figure-of-eight TMS coil, ${ }^{3}$ while animals were anesthetized with isoflurane (1.5\%). Prior to the start of rTMS treatment, the resting motor threshold was determined for each rat. This was done by stimulating the forelimb region of the brain with single TMS pulses, while simultaneously recording muscle activity of the musculus brachialis with electromyography (based on the protocol described in Boonzaier et al. ${ }^{3}$ ). During stimulation, both the TMS coil and the animals were fixed in a stereotaxic frame to allow for accurate coil placement and stimulation. The animals either received high-frequency $(5 \mathrm{~Hz})$, low-frequency $(1 \mathrm{~Hz})$ or sham stimulation. Repetitive TMS treatment was given at $85 \%$ of the rat's resting motor threshold for the high- and low-frequency treatment groups, whereas stimulation for the sham group was applied at $10 \%$ of the resting motor threshold intensity. Each rTMS treatment session lasted for 20 minutes.

MRI

\section{Patients}

MRI scans were acquired at the University Medical Center Utrecht using a Philips 3T Achieva scanner. 3D T1-weighted anatomical MRI scans for lesion delineation were acquired at 5-6 weeks ( $n=7), 3$ months ( $n=1), 6$ months $(n=1)$ and/or 12 months $(n=2)$ after stroke. Anatomical MRI paramters were: repetition time $(T R)=8.13 \mathrm{~ms}$, echo time $(T E)=3.7 \mathrm{~ms}$, flip angle $=8^{\circ}$, field-of-view $($ FOV $)=512 \times 512$, spatial resolution: $0.47 \times 0.47 \times 1.0 \mathrm{~mm}^{3}$ (total scan time $=2.5 \mathrm{~min}$ ). The images were resized to an isotropic voxel size of $1 \mathrm{~mm}^{3}$ before manual delineation of the lesions. SPM12 was used to inverse normalize a cerebral mask in $\mathrm{MNI}$ space to individual patient space in order to calculate individual cerebral hemispheric volumes. 


\section{Rats}

MRI was executed on a 9.4 T preclinical MR system (Varian Inc., Palo Alto, CA, USA) while animals were mechanically ventilated with $1.5 \%$ isoflurane in air/ $\mathrm{O}_{2}(4: 1)$. Anatomical images were acquired with a balanced steady-state free precession sequence (250 $\mu \mathrm{m}$ isotropic spatial resolution, $\mathrm{TR}=5 \mathrm{~ms}, \mathrm{TE}=2.5 \mathrm{~ms}$, flip angle $=20^{\circ}, \mathrm{FOV}=40 \times 32 \times 24 \mathrm{~mm}^{3}$, matrix $=160 \times 128 \times 96,3$ averages and pulse angle shifts of $0^{\circ}, 90^{\circ}, 180^{\circ}$ and $270^{\circ}$, total scan time $=12 \mathrm{~min}$ ). The stroke infarction volume was calculated from manual segmentations of lesioned tissue.

\section{References}

1. Watson, B. D., Dietrich, W. D., Busto, R., Wachtel, M. S. \& Ginsberg, M. D. Induction of reproducible brain infarction by photochemically initiated thrombosis. Ann. Neurol. 17, 497-504 (1985).

2. Schmidt, A. et al. Photochemically induced ischemic stroke in rats. Exp. Transl. Stroke Med. 4, 13(2012).

3. Boonzaier, J. et al. Design and Evaluation of a Rodent-Specific Transcranial Magnetic Stimulation Coil: An In Silico and In Vivo Validation Study. Neuromodulation 23, 324-334 (2019). 


\section{Appendix 2.2}

Table S2.1: Movement element rating scale for humans, from Klein et al. ${ }^{1}$

\begin{tabular}{|c|c|c|}
\hline \multicolumn{3}{|c|}{ Human movement elements } \\
\hline Element & Sub-element & Description \\
\hline \multirow[t]{2}{*}{ 1. Orient } & A. Head & $\begin{array}{l}\text { Head is moving freely then fixes on target at } \\
\text { beginning of trial }\end{array}$ \\
\hline & B. Eye & $\begin{array}{l}\text { Eyes locate target prior to movement of head/ } \\
\text { reach }\end{array}$ \\
\hline \multirow[t]{4}{*}{ 2. Lift } & A. Flex elbow & Initial hand lift is due to flexion of the elbow \\
\hline & B. Digits semi-flex & Digits semi-flex \\
\hline & C. Wrist supination & Wrist supinates approximately $30^{\circ}$ \\
\hline & D. Digits to midline & $\begin{array}{l}\text { Tips of digits are brought toward the midline of } \\
\text { the body }\end{array}$ \\
\hline \multirow[t]{3}{*}{ 3. Advance } & A. Limb advance & Hand takes shortest path to target \\
\hline & B. Hand ends at target & Hand stops directly above target \\
\hline & C. Trunk & $\begin{array}{l}\text { Trunk leans to the side opposite reach as hand } \\
\text { approaches the target }\end{array}$ \\
\hline \multirow[t]{3}{*}{ 4. Pronation } & A. Digits open/extend & Digits open and extend over the food target \\
\hline & B. Full hand turn & Knuckle on reaching hand forms horizontal line \\
\hline & C. Elbow extend & $\begin{array}{l}\text { Elbow opens to full arm length as subject } \\
\text { reaches }\end{array}$ \\
\hline \multirow{3}{*}{ 5. Grasp } & A. Pincet grasp & Thumb and index finger grasp food item \\
\hline & B. Digits $3-5$ independent & Digits 3-5 remain still as grasp is executed \\
\hline & C. Wrist extension & Wrist extends to lift food item from platform \\
\hline \multirow[t]{6}{*}{ 6. Supination } & A. Supination I & $\begin{array}{l}\text { Reaching hand supinates } 45^{\circ} \text { immediately after } \\
\text { vertical lift }\end{array}$ \\
\hline & B. Supination II & $\begin{array}{l}\text { Hand supinates another } 45^{\circ} \text { when in close } \\
\text { proximity to mouth }\end{array}$ \\
\hline & A. Hand contacts mouth & $\begin{array}{l}\text { Fingertips contact lips for placement of food } \\
\text { item in mouth }\end{array}$ \\
\hline & B. Digits open & Digits open to release food item into mouth \\
\hline & C. Hand on lap & $\begin{array}{l}\text { Hand is placed on lap with fingers extended and } \\
\text { palm down }\end{array}$ \\
\hline & D. Trunk & Trunk leans back toward midline \\
\hline
\end{tabular}


Table S2.2: Movement element rating scale for rodents, from Moon et al. ${ }^{2}$

\begin{tabular}{ll}
\hline Element & Description \\
\hline Limb lift & $\begin{array}{l}\text { The head and the snout are oriented towards the target so that that rat } \\
\text { sniffs the target } \\
\text { The forepaw is lifted from the floor until the digits are aligned with the } \\
\text { midline of the body } \\
\text { The elbow is adducted to the body midline with a movement of the } \\
\text { upper arm while the digits remain positioned on the body midline. }\end{array}$ \\
Aim & $\begin{array}{l}\text { The forelimb moves forward through the slot } \\
\text { Advance }\end{array}$ \\
Digits open & $\begin{array}{l}\text { The digits are extended as the limb is advanced and then are opened as } \\
\text { The elbow abducts with a movement of the upper arm pronating the paw } \\
\text { over the target in an arpeggio movement }\end{array}$ \\
Grasp & $\begin{array}{l}\text { The arm remains still, while the digits close to grasp the food and then } \\
\text { the paw is extended and raised }\end{array}$ \\
Supination I & The paw is supinated so that the palm faces the mouth \\
Supination II & $\begin{array}{l}\text { The paw is supinated so that the palm faces the mouth } \\
\text { The food pellet is released into the mouth by opening the digits }\end{array}$ \\
Release &
\end{tabular}

\section{References}

1. Klein, A., Sacrey, L. A. R., Whishaw, I. Q. \& Dunnett, S. B. The use of rodent skilled reaching as a translational model for investigating brain damage and disease. Neurosci. Biobehav. Rev. 36, 1030-1042 (2012).

2. Moon, S. K., Alaverdashvili, M., Cross, A. R. \& Whishaw, I. Q. Both compensation and recovery of skilled reaching following small photothrombotic stroke to motor cortex in the rat. Exp. Neurol. 218, 145-153 (2009). 


\section{Appendix 2.3}

\section{Photothrombotic stroke lesion}

Multislice MR images of post-stroke rat brain displayed a focal unilateral lesion in the sensorimotor cortex (Figure S2.4).

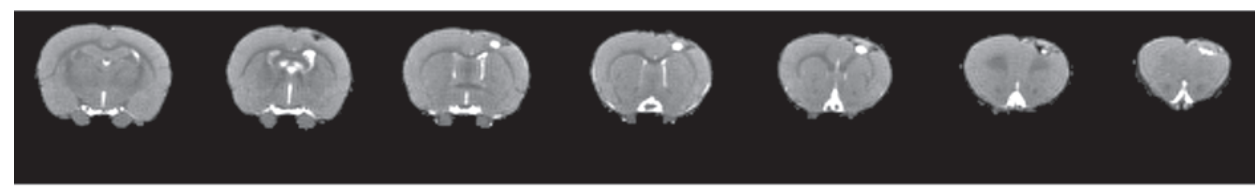

posterior

anterior

Figure S2.4: MRI of stroke lesion.

Anatomical MR images of consecutive coronal rat brain slices at 17 days after photothrombotic stroke, revealing a unilateral lesion in the sensorimotor cortex. MRI data showed no signs of damage to the subcortical striatum. Signal changes were occasionally observed in white matter underlying the lesion, which may be attributed to microhemorrhages.

\section{Skilled reaching}

Skilled reaching success rate, expressed as the percentage of successfully obtained pellets out of $2-20$ reaching trials, dropped from $38 \pm 17 \%$ before stroke to $17 \pm 16 \%$ at 3 days after stroke in rats (Figure S2.5). Partial recovery to $23 \pm 20 \%$ was observed after 23 days.

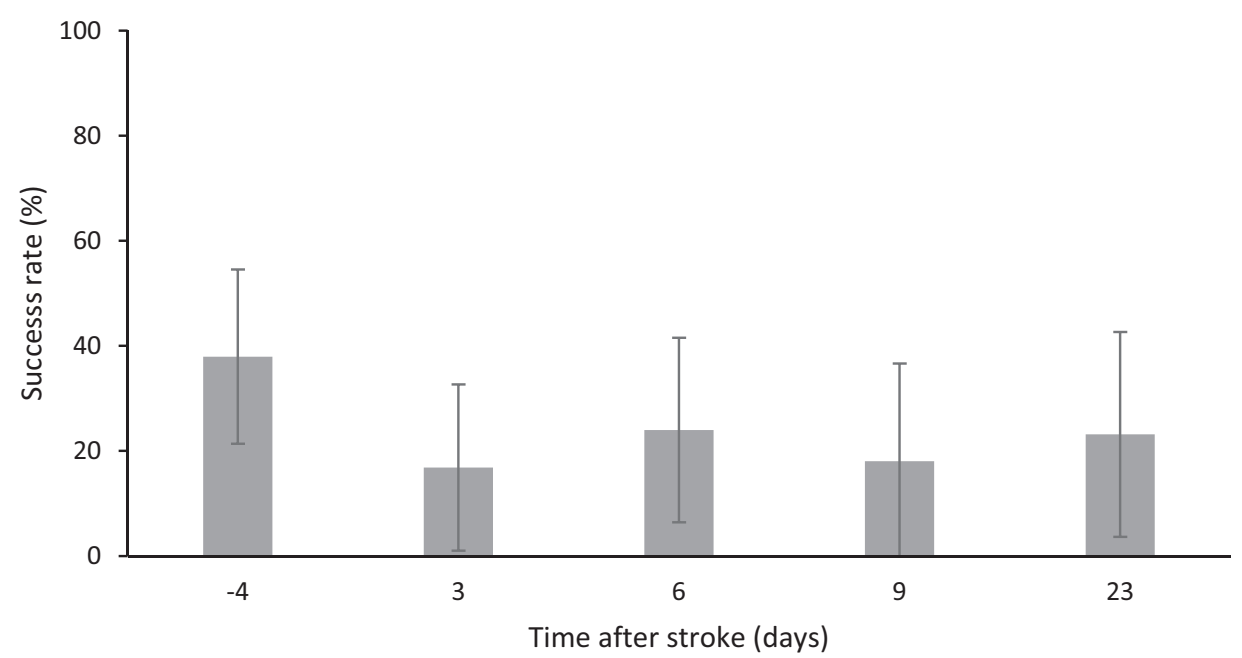

Figure S2.5: Pellet reaching success rate at different time-points before and after stroke in rats. Data are presented as means \pm standard deviation. 


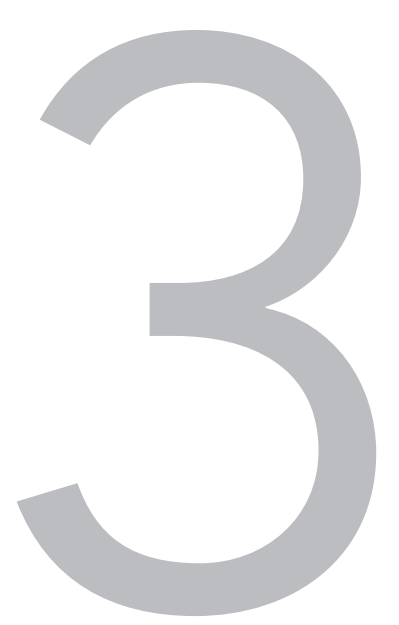

\section{Comparison of self-reported versus observational clinical measures of improvement in upper limb capacity in patients after stroke}

Eline C.C. van Lieshout Johanna M.A. Visser-Meily

Rinske H.M. Nijland

Rick M. Dijkhuizen

Gert Kwakkel 


\section{Abstract}

Background: Recovery of the paretic arm post-stroke can be measured by observational and self-reported measures. This study aims to determine whether correspondence (match) or non-correspondence (mismatch) between observational and self-reported improvements of upper limb capacity is significantly different 0-3 compared to 3-6 months post-stroke.

Methods: A total of 159 patients with ischemic stroke with upper limb paresis were included in the study. Recovery of arm capacity was measured with observational (Action Research Arm Test; ARAT) and self-reported measures (Motor Activity Log Quality of Movement; MAL-QOM and Stroke Impact Scale Hand; SIS-Hand) at 0-3 and 3-6 months post-stroke. Proportion matches was defined (contingency tables and Fisher's exact test) and compared across the different time-windows using McNemar's test.

Results: The proportion matches was not significantly different at 0-3 months compared with 3-6 months post-stroke for the ARAT versus MAL-QOM and SIS-Hand (all p > .05). In case of mismatches, patients' self-reports were more often pessimistic (86\%) in the first 3 months post-stroke with the subsequent 3 months (39\%).

Conclusions: The match between observational and self-reported measures of upper limb capacity is not dependent on timing of assessment post-stroke. Assessment of both observational and self-reported measures may help to recognize possible over- or underestimation of improvement in upper limb capacity post-stroke.

\section{Lay abstract}

One of the most common motor disturbances after stroke is a paretic arm, which may be of little functional use in activities of daily living. Recovery of the paretic arm can be measured by a clinician (observational) or by the patients (patient-reported). It might be expected that observational and patient-reported measures will be strongly related to each other. The aim of this study was to determine whether the correspondence (matches) between those measures is different at 0-3 months post-stroke compared with 3-6 months post-stroke. The results showed that the time-frame post-stroke (0-3 or 3-6 months) did not seem to influence the correspondence between the observational and self-reported measures: there were more matches than mismatches found. Self-reported measures can be used in addition to the observational measures to assess arm recovery. Information on the ability and use of the affected arm outside the treatment setting is valuable for the clinicians, as it provides more insight into the patients' perspective. 


\section{Introduction}

Upper limb paresis is common after stroke and reduces a patient's independence, performance of activities of daily living (ADL) and self-reported quality of life. ${ }^{1}$ Different instruments can be used to assess the upper limb after stroke, based on the levels of function, activity (capacity and performance) and participation (World Health Organization's International Classification of Functioning, Disability and Health (WHO-ICF)). ${ }^{2}$ Upper limb function and capacity can be scored by a clinician using standardized validated measurements. However, a number of these measurements have floor and ceiling effects. In addition, self-perceived difficulties with arm use are not reflected by these clinical performance tests. ${ }^{3}$ Self-reported upper limb outcome measures, however, require subjective assessment of arm functioning at the activity and participation level, as perceived by patients themselves. ${ }^{2,4}$

Recently, self-reported outcome measures (i.e. patient reported outcome measures (PROMs)) have received increasing attention in mapping a patients' perceived recovery in medical practice. ${ }^{5} \mathrm{PROM}$ can provide valuable insights into a patient's status outside the treatment setting, and can detect change in a patient's perceived health status. ${ }^{6-8}$ However, the use of PROMS for stroke patients may suffer from confounding factors, such as neglect, selfawareness, mood, fatigue, social support, relationships, and encouragement from others, which may influence patients' expectations and might lead to under- or over-estimations in self-reported assessments. ${ }^{9-13}$

Moderate correlations have been shown between capacity measures and self-reported measures. ${ }^{14,15}$ To date, only a few cross-sectional studies have investigated the correspondence (match) and non-correspondence (mismatch) between outcomes on observational and selfreported upper limb measures. ${ }^{10,12,16-18}$ As the time course of upper limb recovery is non-linear and driven by poorly understood processes of neural recovery and compensation strategies, outcomes between observational and self-reported measures may deviate, depending on the timing of assessment post-stroke.

Therefore, main aims of the present study were: 1) to determine whether the proportion matches between observational and self-reported improvements on upper limb capacity differs between the early (0-3 months) and late subacute stages 3-6 months); and 2) to identify whether the self-reported improvements may under- or over-estimate the observationally measured improvements of upper limb capacity at these stages.

For the first aim, we hypothesized that there would be a higher proportion of matches at 3-6 months post-stroke than at 0-3 months post-stroke, because patients gradually learn to deal better with and gain more experience of real-world limitations, with a better understanding of their own capabilities. ${ }^{19-21}$ For the second aim, it was hypothesized that 
more patients would overestimate ('optimistic') than underestimate ('pessimistic') their self-reported capacity at 0-3 months post-stroke than at 3-6 months post-stroke, because spontaneous neurological and functional recovery occurs within the first three months poststroke. This can result in more subjectively experienced improvement than observationally measured improvement. ${ }^{22,23}$

\section{Methods}

Data for this study were collected during the EXPLICIT (EXplaining PLastICITy) Stroke trial. ${ }^{24}$ The EXPLICIT Stroke trial was a multicentre, observer-blinded randomized controlled trial to investigate the effects of modified constraint-induced movement therapy (mCIMT) and electromyography-triggered neuromuscular stimulation (EMG-NMS) on upper limb capacity. Eligible patients were screened and included in the first week post-stroke. The included patients had an upper limb paresis and were stratified into a poor (EMG-NMS) and favourable prognosis group (mCIMT) for upper limb recovery. Full details about randomisation, treatment, and study design can be found elsewhere. ${ }^{24}$ The baseline assessments were performed within 2 weeks post-stroke. The data used in this study were taken from baseline, 12 and 26 weeks after stroke onset.

The EXPLICIT Stroke trial ${ }^{24}$ was approved by the Medical Ethical Reviewing Committees of the Leiden University Medical Centre (main reviewing committee: Dutch Central Committee on Research Involving Human Subjects, CCMO, protocol number NL21396.058.08), the VU Medical Centre Amsterdam, the Radboud University Medical Centre Nijmegen, and the University Medical Centre Utrecht in the Netherlands. This trial is registered in the Netherlands Trial Register (NTR, http://www.trialregister.nl, NL1366).

\section{Participants}

All included patients met the following criteria: 1) first-ever, ischemic stroke in one of the cerebral hemispheres; 2) upper limb paresis according to National Institutes of Health Stroke Scale (NIHSS) item 5; 3) baseline ARAT score of $\leq 53$ on a maximum of 57 points; 4) ability to communicate and comprehend (mini mental state examination $\geq 23$ points on a maximum of 30 points); 5) ability to sit independently for at least 30 seconds; 6) $18-80$ years of age; 7) no successful thrombolysis therapy resulting in upper limb motor recovery and attaining 0 points on NIHSS item 5 of the paretic arm; 8) no musculoskeletal impairments of the upper paretic limb; 9) no additional therapies such as botulinum toxin injections or medication intake that may influence upper limb function in the previous 3 months; 10) willing to participate in an intensive rehabilitation treatment program; and 11) written informed consent. 


\section{Observational clinical testing}

The current study used the ARAT as observational measure of upper limb capacity. ${ }^{25}$ Observational measures require an independent assessor trained to measure a patient's skills to perform the tasks in the test. The ARAT assesses the ability to perform gross movements and the ability to grasp, move and release objects differing in size, weight and shape (WHOICF, Activity level). ${ }^{2}$ The items are rated on 4-point scales (0-3), with a maximum score of 57 (best performance)..$^{25}$ The ARAT is a reliable, valid and responsive test ${ }^{26}$ in patients with stroke with mild to moderate motor severity and in the absence of severe cognitive impairment. The Minimal Clinically Important Difference (MCID) was set at 6 points, based on clinical experience and estimates, which is about $10 \%$ of the maximum score. ${ }^{27}$

\section{Self-reported testing}

Dutch versions of the MAL-QOM and SIS-Hand were used to describe the motor performance from the viewpoint of the patient (WHO-ICF, Activity level).

A Dutch version of the 14-item MAL was used to assess how well (Quality of Movement; QOM scale) the paretic arm was used spontaneously during 14 activities of daily living outside the laboratory. The patient is asked to indicate how well he/she used his/her affected arm during certain activities in the past week (e.g. pick up a glass, comb your hair, button a shirt). A 6-point ordinal scale (range 0-5) was used, in which half ratings can also be given. A higher score indicates better performance: maximum score: 5 (transformed scale: overall score (0-70) divided by 14 , resulting in a $0-5$ scale)). This 6 -point scale contains scoring from 'The weaker arm was not used at all for that activity (never)', to 'The weaker arm was used for the purpose indicated but movements were slow or were made with only some effort (fair), to 'The ability to use the weaker arm for that activity was as good as before the stroke (normal)'. Reliability and validity of the MAL has been shown. ${ }^{14}$ A MCID of 0.5 points was used, based on clinical experience and estimates, which reflects $10 \%$ of the maximum score. ${ }^{14}$

Version 3.0 of the SIS is a stroke-specific, self-report, health status measure containing eight domains related to hand function, strength, activities of daily living, communication, emotion, memory and thinking. The SIS is a valid and reliable measure for a diverse group of stroke survivors. ${ }^{28}$ The Hand domain of the SIS consists of five questions and each item is scored on a 5-point Likert scale (transformed from 5-25 to a scale from 0-100). In the questions patients must rate how difficult it was to use their affected hand in a range of activities in the past two weeks (e.g. turn a doorknob, tie a shoelace). Higher scores indicate a low(er) impact of hand problems on health and life. A MCID of 10 points was used, based on clinical experience and estimates, i.e. $10 \%$ of the maximum score. ${ }^{29}$ 


\section{Data analysis}

To calculate change scores for the time-window 0-3 months post-stroke, the baseline scores from the ARAT, MAL-QOM and SIS-Hand were subtracted from the follow-up scores at 3 months. Some of the patients had a baseline score less than a MCID short of the maximum score on one of the outcome measures. Therefore, reaching the maximum score at the follow-up measurement 3 months post-stroke was considered a clinically meaningful change. Change scores for the time-window 3-6 months post-stroke were computed by subtracting the follow-up scores measured at 3 months post-stroke from the follow-up scores 6 months post-stroke. Changes were marked as successful when maximum scores were reached at follow-up, or when changes were beyond the known MCID (10\% of the maximum score). Change scores smaller than the MCID were marked as unsuccessful. Subsequently, patients with a successful change (improvement) on the ARAT as well on a self-reported measure (MAL-QOM and SIS-Hand), and patients with unsuccessful changes (no improvement) on the ARAT and on a self-reported measure (MAL-OOM and SIS-Hand) were grouped as matchers (i.e. true positives and true negatives). Patients with a successful change on the ARAT, but not on a self-reported measure (MAL-QOM and SIS-Hand), and vice versa, were grouped as mismatchers.

Fisher's exact test was used to examine the significance of the association between matches and mismatches (i.e. overall fraction correct). The tested null hypothesis was that a successful and unsuccessful change on the ARAT is equally likely to have a successful change on the MAL-QOM or SIS-Hand. The percentage of false negatives reflects the degree of underestimation, i.e. observed change on the ARAT, without reported change on the MAL-QOM or SIS-Hand.) The percentage of false positives reflects the degree of overestimations, i.e. reported change on the MAL-QOM or SIS-Hand, without observed change on the ARAT. The percentages false positives and false negatives were deduced from the contingency tables. In addition, using the two-way contingency tables, the sensitivity, specificity, positive and negative predicted values (i.e. the probability that an event is present/not present, when the event is present/not present), and, overall fraction correct (i.e. the probability that an event is correctly classified) were also calculated.

Finally, McNemar's test was used to compare the proportions of matches to mismatches between 0-3 and 3-6 months post-stroke, and the association between the ARAT versus MAL-QOM and SIS-Hand. Only those patients were included from whom data were collected at all time-points. The statistical software SPSS 25.0 (SPSS, Chicago, IL, USA) was used for statistical analysis. The level of statistical significance was set two-tailed at $p<.05$. 


\section{Results}

\section{Patient characteristics}

For the EXPLICIT Stroke trial 159 patients were selected (for flow diagram see Appendix 3.1). ${ }^{29}$ There were no reports of adverse effects from the trial. Table 3.1 shows the main characteristics of the included patients at baseline, and average scores on the used outcome measures for different time points post-stroke. Missing data-points from patients on one of the time-points resulted in lower number of total patients in the analyses.

Table 3.1: Patient characteristics measured at baseline

\begin{tabular}{|c|c|c|c|}
\hline \multicolumn{4}{|l|}{ Characteristics } \\
\hline Female/Male, $\mathrm{n}$ & $63 / 96$ & & \\
\hline Age, years mean (SD) & $60.0(12.3)$ & & \\
\hline Affected hemisphere, right/left, $n$ & $105 / 54$ & & \\
\hline Affected dominant side, $\mathrm{n}$ & 54 & & \\
\hline Handedness, R/L & $139 / 17$ & & \\
\hline $\begin{array}{l}\text { Time between stroke onset and baseline assessment } \\
\text { (days, mean (SD)) }\end{array}$ & $8.3(4.1)$ & & \\
\hline Level of education (low/high), n & $113 / 28$ & & \\
\hline Partner (yes/no), n & $111 / 48$ & & \\
\hline Mood (below/above normative score), $\mathrm{n}$ & $89 / 45$ & & \\
\hline Dexterity (minimal/some), $\mathrm{n}$ & $118 / 41$ & & \\
\hline MI (0-100), mean (SD) & $30.3(30.0)$ & & \\
\hline \multirow[t]{2}{*}{ FMA (0-66), mean (SD) } & $18.6(18.9)$ & & \\
\hline & Baseline & 3 months & 6 months \\
\hline ARAT (0-57), mean (SD) & $8.6(13.4)$ & $24.6(22.3)$ & $27.6(23.1)$ \\
\hline SIS-Hand (0-100), mean (SD) & $5.9(2.8)$ & $36.2(40.1)$ & $42.8(42.3)$ \\
\hline MAL-QOM (0-5), mean (SD) & $0.3(0.6)$ & $1.4(1.5)$ & $1.6(1.6)$ \\
\hline
\end{tabular}

MI: Motricity Index; ARAT: Action Research Arm Test; FMA: Fugl-Meyer assessment of the arm; SISHand, Stroke Impact Scale Hand; MAL-QOM, Motor Activity Log-Quality of Movement; SD: standard deviation; n: total number of patients.

Proportion of matches between observational and self-reported measures in the first six months post-stroke

For the time-window 0-3 months post-stroke, $88 \%$ of the patients showed matches on the ARAT versus MAL-QOM, and $89 \%$ on the ARAT versus SIS-Hand (Table 3.2). A successful and unsuccessful change on the ARAT is not equally likely to have a successful change on the MAL-QOM and SIS-Hand ( $p<0.05)$. The sensitivity, specificity, positive- and negative predicted value were comparable for the MAL-QOM and SIS-Hand in comparison to the ARAT. In the time-period 3-6 months post-stroke, a successful and unsuccessful change 


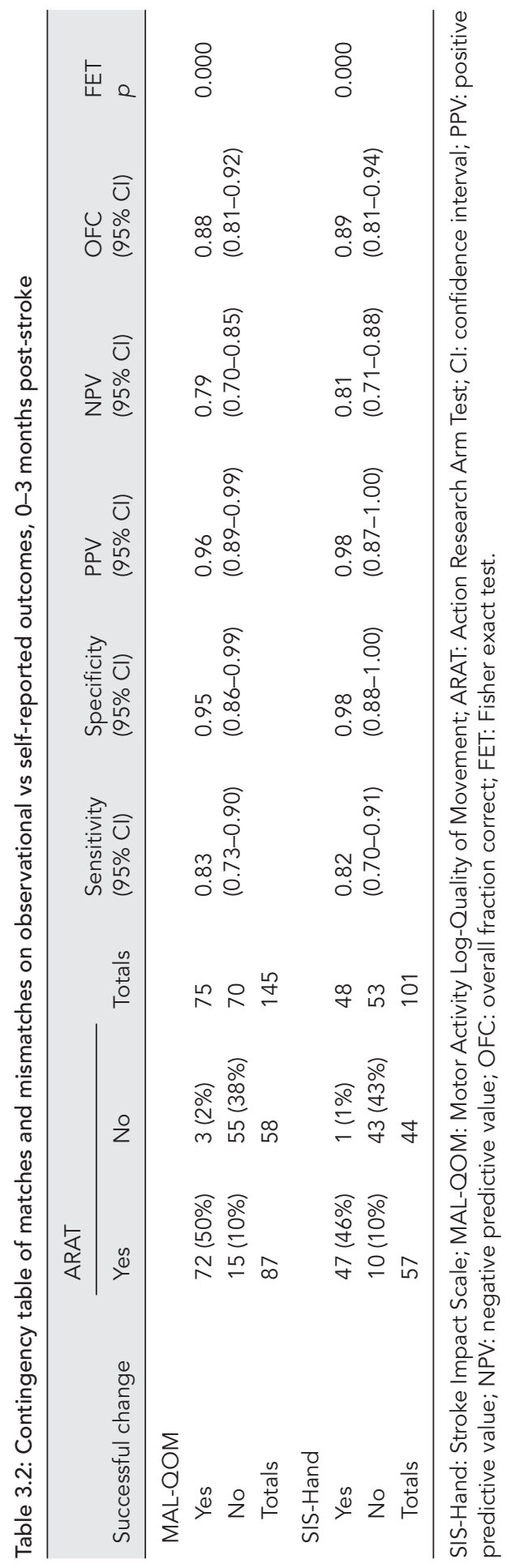


on the ARAT was not equally likely to match a successful change on the MAL-QOM and SIS-Hand ( $p<0.05$ ) (Table 3.3). 83\% of the patients had a match on the ARAT versus MALQOM score, and $81 \%$ had a match on the ARAT versus SIS-Hand. The sensitivity, specificity, positive- and negative predicted values were all slightly lower for the ARAT versus SIS-Hand than the ARAT versus MAL-QOM.

False negatives, i.e. underestimations, were measured in 15 out of 18 patients (83\%) with mismatches for the ARAT versus MAL-QOM in the time-window 0-3 months poststroke (Table 3.2, Figure 3.1). Three patients (2\%) could be classified as false positives, i.e. overestimations. For the ARAT versus SIS-Hand, underestimations were measured in 10 out of 11 patients (91\%). One patient (1\%) could be classified as a false positive (Table 3.2, Figure 3.2). False-positives, i.e. overestimations, were more common (14 out of 24 patients: 58\%) in the mismatch proportion in the time-window 3-6 months post-stroke for the ARAT vs MAL-QOM (Table 3.3, Figure 3.1). For the ARAT vs SIS-Hand, false-positives were measured in 14 out of 22 patients (64\%) (Table 3.3, Figure 3.2).

Within the first three months and beyond the first three months post-stroke, the accuracy (overall fraction correct) was comparable for the MAL-OOM and SIS-Hand in relation to the ARAT (Table 3.2 and 3.3).

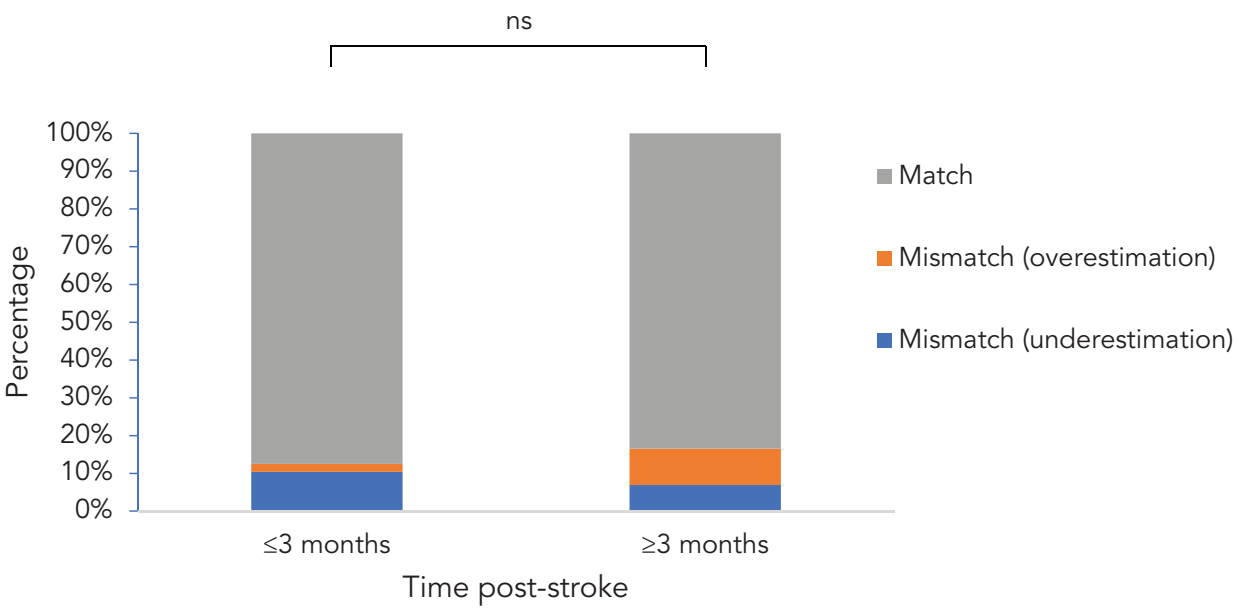

Figure 3.1: Proportion of matches to mismatches between ARAT and MAL-QOM.

Comparison between 0-3 and 3-6 months post-stroke in proportion matches to mismatches

For the ARAT and MAL-QOM, 3-6 months, the number of matches had decreased from $126(87.5 \%)$ to 121 (84\%), which was a non-significant difference ( $p=.487$ ) (Figure 3.1). For the ARAT and SIS-Hand, the proportion of matches decreased from a 0-3 months post- 


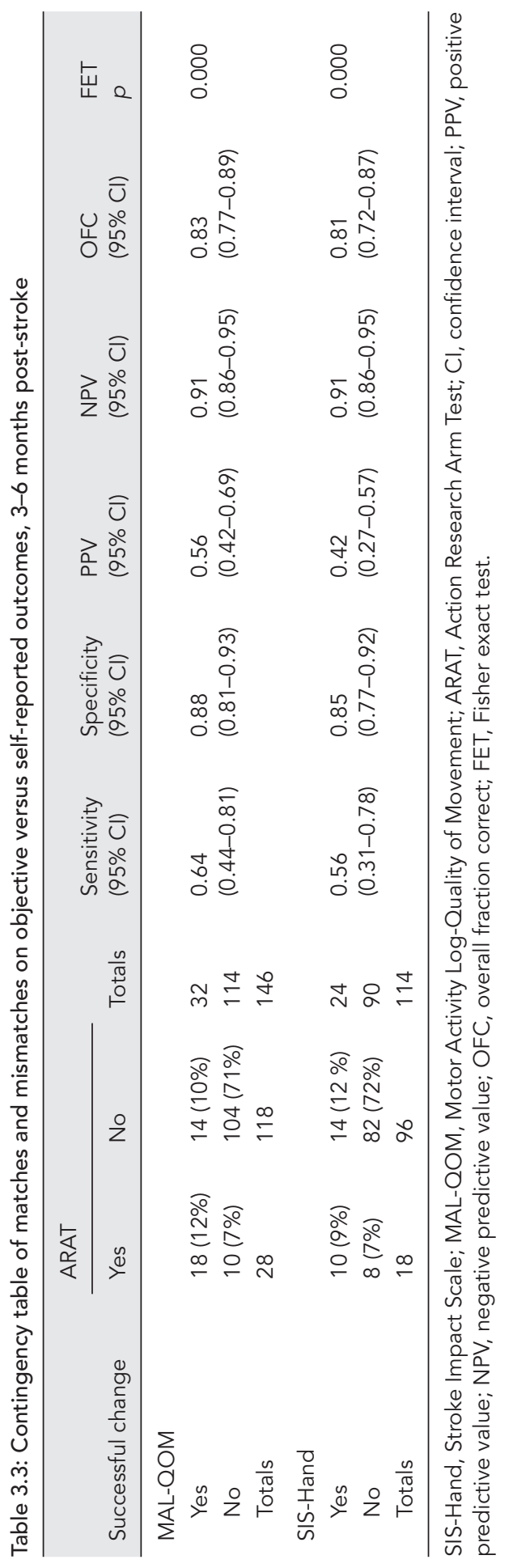




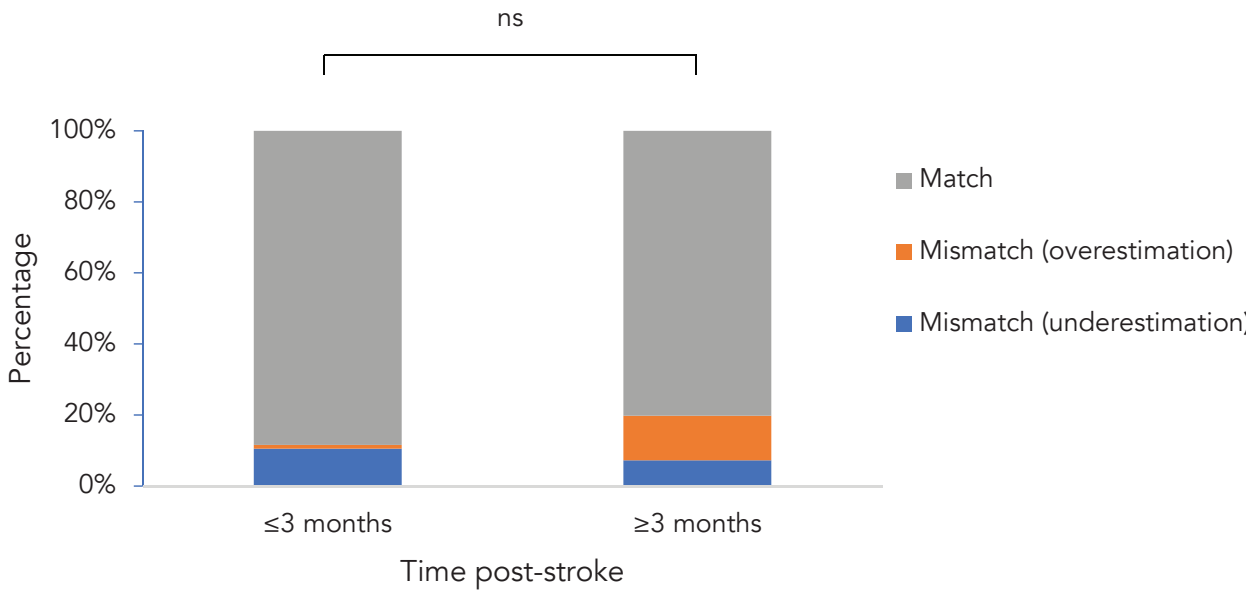

Figure 3.2: Proportion of matches to mismatches between ARAT and SIS-Hand.

stroke percentage of $88 \%$ to $81 \%$ 3-6 months, which was a non-significant difference ( $p=$ .210) (Figure 3.2). This change was a consequence of seven matches within three months post-stroke changing into mismatches beyond three months. The sensitivity, specificity, positive predicted value, except the negative predicted value, had higher values in the time-window 0-3 months post-stroke.

\section{Discussion}

These results show that stroke patients had significantly more matches than mismatches between observational and self-reported measures of improvements of the upper limb during the first 6 months, which is in accordance with earlier findings. ${ }^{10,16,17,30}$ Contrary to our hypothesis, the proportion of matches remained stable between 0-3 months and 3-6 months post-stroke, and is therefore not significantly dependent on the timing of assessment within the subacute stage post-stroke. Patients with mismatches within the first three months poststroke were more likely to underestimate their self-reported performance (86\%), whereas between 3-6 months they tended to overestimate their self-reported performance (61\%) on the MAL-QOM and SIS-Hand domain, compared to their actual improvements on the ARAT.

The significantly high correspondence between observational and self-reported improvements of the upper limb are in line with cross-sectional studies in which significant associations between observational and self-reported measures were found. 10,16,17,30 These findings suggest that the patient's perspective is usable in the evaluation of upper limb rehabilitation, which supports patient involvement in rehabilitation as encouraged in patient-centred care. 
In line with the present study, van Delden et al. (2013) ${ }^{10}$ used the MCID of the change scores to determine an improvement in scores, and found a significant discrepancy in the proportions of matches, compared to mismatches, between the ARAT and MAL-QOM, where matches were more prevalent. However, in contrast, they found no significant difference in the proportion of matches and mismatches between the ARAT and SIS-Hand. This may be explained by the differences in severity and timing of assessments of upper limb paresis between both studies. Van Delden et al. (2013) ${ }^{10}$ only included patients with noticeably preserved motor function (i.e. control of the paretic wrist and fingers) in contrast to the current study, in which $63.5 \%$ of the patients could not voluntarily extend the thumb and/or two or more fingers. Since preserved control in hand function (i.e. finger extension) early post-stroke is a favourable sign for good outcome of the paretic upper limb, more spontaneous motor recovery is expected and needs to be perceived and subsequently quantified by the patient, which can complicate self-reports and may result in mismatches. ${ }^{31}$ Otherwise, the group of patients that no longer recovers remains stable, which can facilitate self-reports and may result in matches.

Neither of the self-reported measures (MAL-QOM and SIS-Hand) was found to be superior in terms of the number of matches with the observational measure (ARAT) during the sixmonth period after stroke. However, beyond the first three months the values for sensitivity, specificity, positive- and negative predicted value were slightly lower than within the first three months post-stroke. The MAL-QOM and SIS-Hand have not been compared previously. ${ }^{14,15}$ However, a possible explanation for higher sensitivity, specificity values in the first three months post-stroke is a higher degree of neuroplasticity early after stroke. Since larger percent changes are required for a self-reported measure as the MAL-QOM to exceed the measurement error, the sensitivity, specificity values can be lower beyond the first three months post-stroke where less recovery is expected. ${ }^{23,32}$

The proportion of matches remained similar between the early subacute phase (i.e. the first three months post-stroke) and the late subacute phase (3-6 months post-stroke). In measures of self-reported physical function, response shifts seem to occur (i.e. changes within patients regarding internal standards, values or conceptualization of health-related quality of life) over time post-stroke. 'Evaluation-based' items, such as when the patient need to evaluate their difficulty in task performance, are most susceptible to response shifts. ${ }^{33}$ The SIS-Hand and MAL-QOM contain evaluation-based items. Although patients that have recalibrated what difficulty means to them, it corresponded with the observationally detected improvements. These findings, while preliminary, provide further support for the use of PROMs in the assessment of upper limb capacity. However, caution must be applied, as the findings might be different for patients with severe communication problems or cognitive complaints. This group of patients was not included in our study. 
The mismatches (over- and under-estimations) between observational and self-reported outcome measures could be attributed to different causes. Underestimations of self-reported capacity in the first three months may be associated with a more pronounced disturbed self-awareness, ${ }^{20}$ a limited insight into one's own functioning, ${ }^{19}$ more negativity-prone thoughts, and lack of information about the rehabilitation phase. Another possibility is that the improved, but affected, upper limb capacity is insufficiently used in daily activities, so that functional recovery is not fully experienced. Overestimations of self-reported capacity beyond the first three months may be explained by a less disturbing perception of upper limb impairments or better adaptation to the new situation. ${ }^{33}$

Other reasons for mismatches between observational- and self-reported outcome measures might be that standardized testing does not account for complex and stressful real-world situations in contrast to perceived self-reported outcomes. Reverse conditions may also be possible, where patients adapt to their own environment and use compensatory strategies to manage daily life, despite poorer performance in a single (test) environment. ${ }^{34,35}$

\section{Study limitations}

This study has some limitations. Firstly, this study had a restricted sample size, and the outcome measures were arbitrarily chosen based on presence in the EXPLICIT trial. ${ }^{24}$ Secondly, there is no consensus about the most appropriate methodological method to identify (clinical meaningful) improvement (e.g. MCID values, cut-off scores). We chose to use the MCID values (based on clinical experience and expertise; $10 \%$ of the total range of the scale) of the outcome measures to define if a given improvement between two time points was smaller (unsuccessful change) or larger (successful change) than these values. ${ }^{27}$ In addition, different methods and algorithms are also used to calculate MCIDs (i.e. distributionbased or anchor-based approaches, clinical experience and expertise). Thirdly, patients with severe communication problems and cognitive deficits were excluded from the EXPLICIT trial. In particular, this group of patients run the risk of inaccurate self-reports, which limits the generalizability of the results. ${ }^{9-11}$

\section{Conclusion}

Self-reported questionnaires used for monitoring upper limb recovery are accurate when compared to observationally measured improvement in the early and late subacute phase after stroke. The current study suggests that timing of assessment post-stroke does not affect the accuracy of self-reports in sub-acute stages. Self-reported measures can give additional insights into the impact of disability on the patient, beyond what is provided by observational measures alone. Self-reported measures in addition to observational measures 
can help to design optimized rehabilitation strategies for patients who underestimate their capacity (training in use of the affected hand, positive psychology, self-efficacy, expectations management). For the patients who overestimate their capacity training in body-image may be warranted.

In order to include patients with severe communication problems or cognitive deficits in PROMS, further research should focus on determining whether alternative self-reported measures and reported data by a proxy are equally accurate as observational measures.

\section{Funding}

This work was supported by the Netherlands Organization for Scientific Research $(\mathrm{VICl}$ 016.130.662).

\section{References}

1. Lai, S. M., Studenski, S., Duncan, P. W. \& Perera, S. Persisting consequences of stroke measured by the stroke impact scale. Stroke 33, 1840-1844 (2002).

2. World Health Organization (WHO). International classification of functioning, disability and health. World Health Organization 1-15 (2003). Available at: http://www.who.int/classifications/icf/ icfchecklist.pdf?ua=1.

3. Kwakkel, G. Standardised measurement of sensorimotor recovery in stroke trials: consensus-based core recommendations from the Stroke Recovery and Rehabilitation Roundtable (SRRR). Int. J. Stroke 12, 451-461 (2017).

4. Ekstrand, E., Rylander, L., Lexell, J. \& Brogårdh, C. Perceived ability to perform daily hand activities after stroke and associated factors: A cross-sectional study. BMC Neurol. 16, 1-9 (2016).

5. Reeves, M. et al. Patient-Reported Outcome Measures (PROMs) for Acute Stroke: Rationale, Methods and Future Directions. Stroke 49, 1549-1556 (2018).

6. Stewart, J. C. \& Cramer, S. C. Patient-reported measures provide unique insights into motor function after stroke. Stroke 44, 1111-1116 (2013).

7. Uswatte, G., Taub, E., Morris, D., Light, K. \& Thompson, P. A. The Motor Activity Log-28: Assessing daily use of the hemiparetic arm after stroke. Neurology 67, 1189-1194 (2006).

8. Blennerhassett, J. M., Avery, R. M. \& Carey, L. M. The test-retest reliability and responsiveness to change for the Hand Function Survey during stroke rehabilitation. Aust. Occup. Ther. J. 57, 431-438 (2010).

9. Coenen, M. et al. Do patient-reported outcome measures capture functioning aspects and environmental factors important to individuals with injuries or disorders of the hand? J. Hand Ther. 26, 332-342 (2013).

10. van Delden, A. (Lex) E. Q., Peper, C. (Lieke) E., Beek, P. J. \& Kwakkel, G. Match and mismatch between objective and subjective improvements in upper limb function after stroke. Disabil. Rehabil. 35, 1961-1967 (2013). 
11. Hochstenbach, J., Prigatano, G. \& Mulder, T. Patients' and relatives' reports of disturbances 9 months after stroke: Subjective changes in physical functioning, cognition, emotion, and behavior. Arch. Phys. Med. Rehabil. 86, 1587-1593 (2005).

12. Persson, H. C., Danielsson, A. \& Sunnerhagen, K. S. A cross sectional study of upper extremity strength ten days after a stroke; relationship between patient-reported and objective measures. BMC Neurol. 15, 1-7 (2015).

13. Essers, B. et al. Mismatch between observed and perceived upper limb function: an eye-catching phenomenon after stroke. Disabil. Rehabil. 41, 1545-1551 (2019).

14. Van Der Lee, J. H., Beckerman, H., Knol, D. L., De Vet, H. C. W. \& Bouter, L. M. Clinimetric properties of the motor activity log for the assessment of arm use in hemiparetic patients. Stroke 35, 1410-1414 (2004).

15. Beebe, J. A. \& Lang, C. E. Relationships and responsiveness of six upper extremity function tests during the first 6 months of recovery after stroke. J. Neurol. Phys. Ther. 33, 96-103 (2009).

16. Dromerick, A. W. et al. Relationships between upper-limb functional limitation and self-reported disability 3 months after stroke. J. Rehabil. Res. Dev. 43, 401 (2006).

17. van der Lee, J. H. et al. Forced use of the upper extremity in chronic stroke patients. Stroke 30, 2369-2375 (1999).

18. Wu, C., Chuang, L., Lin, K., Chen, H. \& Tsay, P. Randomized Trial of Distributed Constraint-Induced Therapy Versus Bilateral Arm Training for the Rehabilitation of Upper-Limb Motor Control and Function After Stroke. Neurorehabil. Neural Repair 25, 130-139 (2011).

19. Zawadzka, E. \& Domańska, Ł. Assessment of select dimensions of patients' emotional functioning at different time periods after stroke. Appl. Neuropsychol. 21, 87-93 (2014).

20. Prigatano, G. P. \& Johnson, S. C. The three vectors of consciousness and their disturbances after brain injury. Neuropsychol. Rehabil. 13, 13-29 (2003).

21. Cooper, C. L., Phillips, L. H., Johnston, M., Whyte, M. \& Macleod, M. J. The role of emotion regulation on social participation following stroke. Br. J. Clin. Psychol. 54, 181-199 (2015).

22. Dancause, N. \& Nudo, R. J. Shaping plasticity to enhance recovery after injury. Prog. Brain Res. 192, 273-295 (2011).

23. Biernaskie, J. Efficacy of rehabilitative experience declines with time after focal ischemic brain injury. J. Neurosci. 24, 1245-1254 (2004).

24. Kwakkel, G. et al. Impact of early applied upper limb stimulation: the EXPLICIT-stroke programme design. BMC Neurol. 8, 49 (2008).

25. Yozbatiran, N., Der-Yeghiaian, L. \& Cramer, S. C. A standardized approach to performing the action research arm test. Neurorehabil. Neural Repair 22, 78-90 (2008).

26. van der Lee, J. H., Roorda, L. D., Beckerman, H., Lankhorst, G. J. \& Bouter, L. M. Improving the Action Research Arm test: a unidimensional hierarchical scale. Clin. Rehabil. 16, 646-653 (2002).

27. Van der Lee, J. H. et al. The intra- and interrater reliability of the action research arm test: A practical test of upper extremity function in patients with stroke. Arch. Phys. Med. Rehabil. 82, 14-19 (2001).

28. Duncan, P. W., Bode, R. K., Lai, S. M. \& Perera, S. Rasch analysis of a new stroke-specific outcome scale: The stroke impact scale. Arch. Phys. Med. Rehabil. 84, 950-963 (2003).

29. Kwakkel, G. et al. Effects of unilateral upper limb training in two distinct prognostic groups early after stroke. Neurorehabil. Neural Repair 30, 804-816 (2016).

30. Wu, C. Y., Chuang, L. L., Lin, K. C., Lee, S. Da \& Hong, W. H. Responsiveness, minimal detectable change, and minimal clinically important difference of the nottingham extended activities of daily living scale in patients with improved performance after stroke rehabilitation. Arch. Phys. Med. Rehabil. 92, 1281-1287 (2011). 
31. Nijland, R. H. M., Van Wegen, E. E. H., Harmeling-Van Der Wel, B. C. \& Kwakkel, G. Presence of finger extension and shoulder abduction within 72 hours after stroke predicts functional recovery: Early prediction of functional outcome after stroke: The EPOS cohort study. Stroke 41, 745-750 (2010).

32. Simpson, L. A. Functional recovery following stroke: Capturing changes in upper extremity function. Neurorehabil. Neural Repair 27, 240-250 (2013).

33. Barclay-Goddard, R., Lix, L. M., Tate, R., Weinberg, L. \& Mayo, N. E. Health-related quality of life after stroke: Does response shift occur in self-perceived physical function? Arch. Phys. Med. Rehabil. 92, 1762-1769 (2011).

34. Dennis, A. et al. Cognitive context determines dorsal premotor cortical activity during hand movement in patients after stroke. Stroke 42, 1056-1061 (2011).

35. Ten Brink, A. F., Visser-Meily, J. M. A. \& Nijboer, T. C. W. Dynamic assessment of visual neglect: The Mobility Assessment Course as a diagnostic tool. J. Clin. Exp. Neuropsychol. 40, 161-172 (2018). 


\section{Appendix 3.1 Inclusion flow diagram}

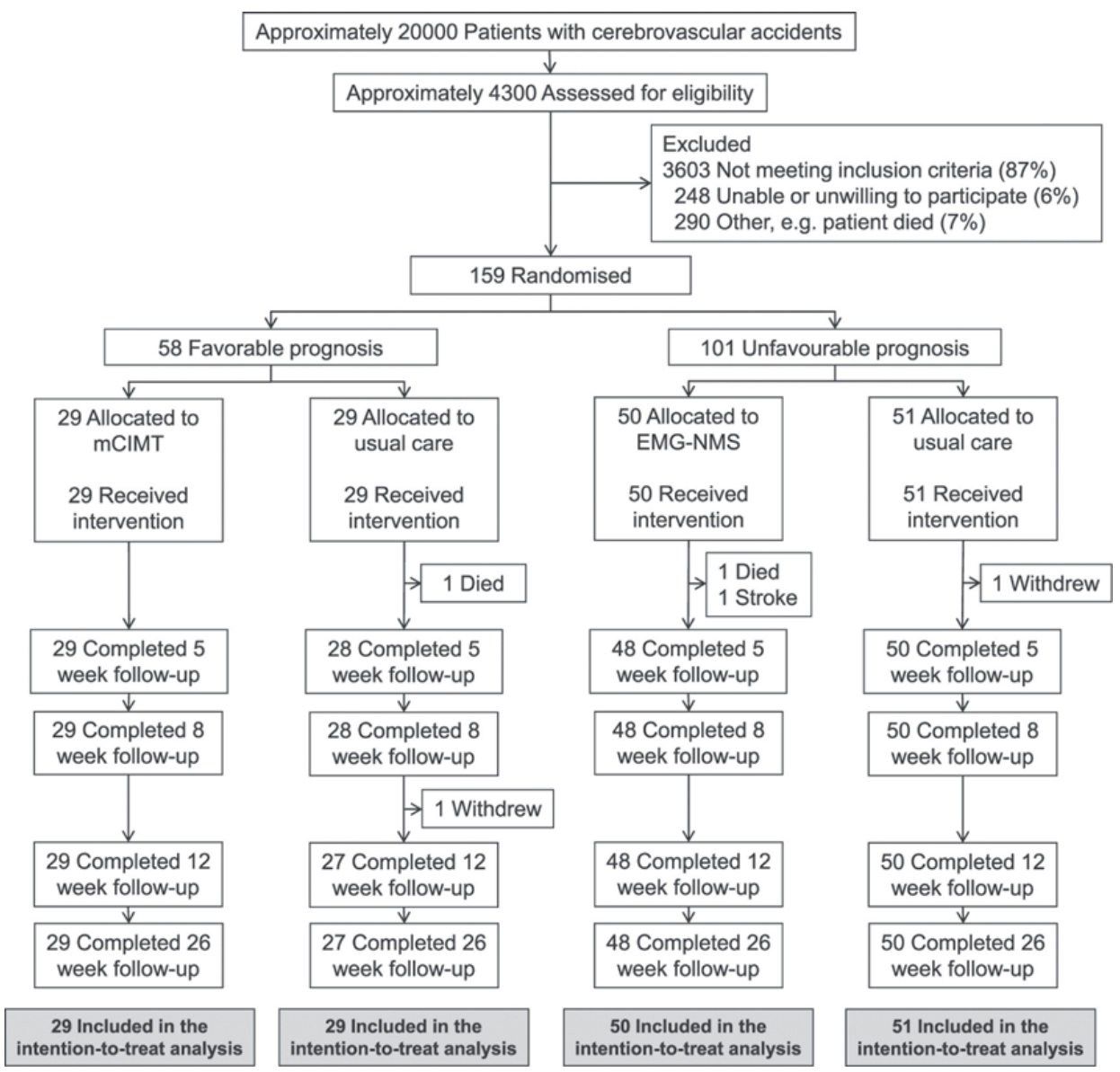




\section{Does upper limb strength play a prominent role in health-related quality of life in stroke patients discharged from inpatient rehabilitation?}

Eline C.C. van Lieshout Ingrid G. van de Port Rick M. Dijkhuizen Johanna M.A. Visser-Meily 


\section{Abstract}

Background: Impairments in arm function are a common problem in stroke survivors and have a large impact on health-related quality of life (HRQoL). Little is known about the longitudinal relationship between recovery of upper limb strength and changes in HRQoL.

Objectives: This study aimed to determine to what extent changes in HRQoL are related to changes in upper limb strength after discharge from inpatient rehabilitation.

Methods: 250 patients from an RCT were assessed at discharge from inpatient rehabilitation (baseline) and at 12 weeks post-discharge (follow-up). The Stroke Impact Scale was used to measure HRQoL, and the Motricity Index Arm was used to measure upper limb strength. Hierarchical regression analysis was performed to determine the predictive value of upper limb strength on HRQoL, relative to demographic and clinical characteristics. Regression analysis was used to determine the relation between upper limb strength improvement and HRQoL improvement.

Results: Upper limb strength at baseline was a major predictor of HRQoL at follow-up, after accounting for demographic and clinical characteristics $(p<.05)$. Improvement in HROoL was positively related to improvement in upper limb strength $(F(1,240)=18.351, p<.0005)$.

Conclusions: These findings highlight the importance of upper limb strength in HRQoL, as $\mathrm{HRQOL}$ is associated with improvement in upper limb strength recovery. Better monitoring of recovery and treatment of upper limb strength during the outpatient rehabilitation period and beyond, i.e. outside the typical time-window of recovery in the first three months poststroke, might contribute to higher quality of life for stroke survivors. 


\section{Introduction}

Stroke is a major health problem across the world causing complex disability. ${ }^{1}$ The impact of this common and serious condition on an individual's life is considerable: physical, psychological and social consequences can be experienced. ${ }^{2-4}$ Upper limb paresis, a muscle weakness in the affected limb to one side of the body, is one of the most frequently occurring conditions (up to $85 \%$ of stroke survivors). ${ }^{5,6}$ Improvement in upper extremity motor function occurs mainly in the first few months after stroke. ${ }^{7}$ At 6 months post-stroke, estimates pointed out that some dexterity in the paretic arm is found in $38 \%$ of the stroke patients who show no dexterity in the first week post-stroke..$^{7-9}$

Arm function plays a critical role in the performance of daily life activities. Most everyday activities require the use of both hands, and because of this the performance of bimanual activities receives considerable attention in the rehabilitation setting. ${ }^{10} \mathrm{Improved}$ arm and hand function positively contribute to societal participation and (health-related) quality of life. ${ }^{1,10,11}$ Health-related quality of life (HRQoL) can be defined as an individual's (or group's) perceived physical and mental health over time. ${ }^{12}$ There is a growing body of literature that recognises that different factors influence $\mathrm{HRQ}$ oL after stroke. 1,4,13,14 A cross-sectional study has shown that the extent of upper limb improvement positively influences a patient's perception of what activities can be performed, which in turn enhances HRQoL. ${ }^{1}$ Incomplete motor recovery of the upper and lower extremities has been found to be the strongest predictor of a lower HRQoL in a observational study. ${ }^{4}$

Whilst some research has been carried out on the association between arm function and HRQoL, ${ }^{1,4,13,14}$ there has been no longitudinal investigation of improvement in HRQoL in relation to improvement in arm recovery. Obtaining insights into this relationship will be useful for understanding problems faced by patients and for planning and optimization of rehabilitation treatment after stroke.

The first aim of the present study was to identify the relation between upper limb strength and HRQoL at discharge from inpatient rehabilitation and at follow-up (12 weeks later). Second, we aimed to determine whether upper limb strength at discharge from inpatient rehabilitation predicts $\mathrm{HRQOL}$ at follow-up, when corrected for patient and stroke characteristics. Third, we aimed to determine whether a change in upper limb strength is related to a change in $\mathrm{HRQ}$ oL over time. We hypothesized that an improvement in upper limb strength is positively related to an improvement in HRQoL. 


\section{Methods}

Data for this study were collected during the FIT-stroke trial. ${ }^{15}$ The FIT-Stroke trial is a stratified, multicentre single-blinded randomised controlled trial to investigate the effectiveness of task-oriented circuit class training. Stroke patients were recruited in nine outpatient rehabilitation centres in the Netherlands, between June 2008 and December 2010. All included patients completed an inpatient rehabilitation period (average of three months) and were included at the start of their outpatient rehabilitation period. The inpatient rehabilitation period consisted of a multidisciplinary approach to reach complex (physical and cognitive) rehabilitation goals. The patients were treated by nurses, physical therapists, occupational therapists, speech therapists, social workers and psychologists. Outcomes from assessments at baseline (start outpatient rehabilitation period) and 12 weeks after randomization were used in this study. Full details of the FIT-Stroke trial have been reported elsewhere. ${ }^{15}$

The FIT-Stroke protocol has been approved by the Medical Ethics Committee of the University Medical Centre Utrecht and all the participating rehabilitation centres. The trial is registered in the Dutch Trial Register (NTR1534). The content of this manuscript conforms to STROBE guidelines. ${ }^{16}$

\section{Participants}

For inclusion, patients were eligible if they 1) had a verified stroke according to the WHO definition; 2) were discharged from a rehabilitation centre; 3) needed to continue physiotherapy during outpatient care to improve walking competency or physical condition, or both; and 4) were able to give informed consent and motivated to participate in a 12 week intensive program of physiotherapy. Patients affected by cognitive deficits (Mini Mental Status Examination, < 24 points), who were not able to communicate (Utrechts Communicatie Onderzoek (UCO), < 4 points) or lived more than 30 kilometres from the rehabilitation centre were excluded. All participants provided written informed consent. The study size was based on a power analysis. ${ }^{15}$

\section{Outcome measures}

Health-Related Quality of Life: Stroke Impact Scale (SIS), version 3

The SIS 3.0 is a multidimensional, self-reported stroke-specific 59-item instrument that assesses $\mathrm{HRQ} \mathrm{L}$ in eight domains related to activities and participation (including strength, memory, emotion, communication, activities of daily living (ADL), mobility, hand function and participation). The SIS has been shown to have good psychometric properties in a group of stroke survivors. ${ }^{17}$ All 59 items are rated using a five-point Likert scale and scored 
from 'unable to complete the item' to 'no difficulty experienced completing the item'. Each domain of the SIS has a range of 0-100 with higher scores indicating better quality of life. An extra question in the recovery domain assesses how much the patient feels that he/she has recovered from his/her stroke. ${ }^{17}$

\section{Independent variables}

\section{Motricity Index (MI), arm}

The upper extremity subscale of the Motricity Index ( $\mathrm{Ml}$ arm) was used to assess muscle strength of the paretic arm. The $\mathrm{Ml}$ arm gives a rapid and reliable measure of the upper limb strength and uses a 6-point ordinal scale, with higher scores indicating better upper limb strength. The MI has been shown to provide good validity and reliability as a tool in stroke research. ${ }^{18}$ Pinch grip, elbow flexion and shoulder abduction were assessed. On each dimension, scores ranged from 0 (no activity) to 33 (maximal muscle force). ${ }^{18}$

\section{Motricity Index (MI), leg}

The lower extremity subscale of the Motricity Index (MI leg) was used to assess muscle strength and voluntary movement of the paretic leg. Ankle dorsiflexion, knee extension and hip flexion were assessed. The scoring system is similar to the Ml arm described above. The MI has been shown to provide good validity and reliability as a tool in stroke research. ${ }^{18}$

\section{Hospital Anxiety and Depression Scale (HADS)}

The HADS is a self-reported measure for assessing anxiety and depression in patients, but it does not provide a specific diagnosis. ${ }^{19}$ It is a 14-item scale with 7 items for anxiety and 7 items for depression. The items use a four-point rating scale and patients can score between 0 and 3 points per item. The HADS has been proven to be a reliable, valid and practical psychological screening tool, with lower scores indicating lower risk of anxiety and/or depression disorders. ${ }^{19}$

\section{Fatigue Severity Scale (FSS)}

The FSS is a self-administered questionnaire with nine questions that examine the perceived severity of fatigue symptoms in different situations in the past week. ${ }^{20}$ The patient indicates to what extent fatigue determines functioning. Scores for each item range from 1 (strong disagreement) to 7 (strong agreement). The FSS is a valid and reliable scale to measure fatigue in stroke. ${ }^{20}$

\section{Demographic and clinical variables}

Age, gender, stroke type, side of hemiplegia and dominant side affected were assessed as potential predicting variables of HRQoL. 


\section{Data analysis}

We statistically tested for differences in change scores on HRQOL, arm- and leg function between the circuit class training group and the usual care group 12 weeks after randomization to determine if both groups could be included in the analysis. SIS total score was the dependent variable in the regression analysis. Age, gender, clinical variables (stroke type, side of hemiplegia and dominant side affected) and clinical scales (MI arm and leg, HADS, FSS) at baseline and at follow-up were considered as independent variables. We tested whether the assumptions for a linear regression analysis were met. Change-frombaseline scores of the SIS and MI-arm were computed to describe the data.

To determine the relationship between HRQoL and upper limb strength, cross-sectionally at baseline and follow-up, univariate regression analysis was performed. Variables demonstrating $p$-values $<0.20$ were included in the hierarchical regression analysis.

To determine how much variance in HRQOL at follow-up was explained by upper limb strength and other demographic and clinical variables at baseline, hierarchical regression analysis was performed. HRQoL at baseline post-stroke was entered as first block into the analysis (to control for the effect of the dependent variable) and upper limb strength as second block. Clinical variables that could be included in the hierarchical regression analysis were combined as the third block and demographic and stroke characteristics were added in the fourth block. Potential predictor variables were examined for collinearity by inspection of the correlation coefficients (no multicollinearity when coefficients $<0.7$ ) and Tolerance/ VIF values (Tolerance needed to be $>0.1$ and VIF values $<10$ ).

Univariate regression analysis was also performed with change-from-baseline scores for $\mathrm{HRQ}$ oL, as dependent variable, and change-from-baseline scores in upper limb strength. Effect sizes were classified as .02, .15 and .35 as small, medium, and large effect sizes. ${ }^{21}$ Significance levels were set at $p=.05$. Statistical analyses were performed with SPSS, version 25.0.

\section{Results}

In total, 250 participants were included in this study (flowchart; Appendix 4.1). Of the 250 included patients, one patient in the circuit training group and seven in the usual care group were excluded from the analysis. Reasons were withdrawal from participation ( $\mathrm{n}=$ $3)$, death from cancer $(n=2)$, and recurrent stroke $(n=2)$, while one patient missed the 12-week assessment visit because of change of address. ${ }^{15}$ The change scores on HRQoL, arm- and leg function from circuit class training group and control group did not differ significantly from each other 12 weeks after randomization. Table 4.1 lists the clinical and demographic characteristics of the patients. The baseline assessment was done 102 days 
(SD 64) post-stroke and the follow up assessment 12 weeks after baseline. The average age in the population was 57 years (SD 10), 65\% ( $n=162)$ was male, the majority $(n=203)$ had an ischemic stroke, and almost half of the patients $(n=116)$ had a right hemisphere lesion. From this table we can see that there are significant improvements in mean scores over time for most clinical variables $(p<.05)$, except the emotion subscale (SIS), FSS and HADS.

Table 4.1: Baseline characteristics $(n=250)$

\begin{tabular}{|c|c|c|c|}
\hline Characteristics & Baseline & Follow-up* & $p$ value \\
\hline Age (years) & $57(10)$ & & \\
\hline Gender, n (\% male) & $162(65)$ & & \\
\hline $\begin{array}{l}\text { Stroke type, n (\%) } \\
\text { Ischemic } \\
\text { Haemorrhagic }\end{array}$ & $\begin{array}{l}203(81) \\
47(19)\end{array}$ & & \\
\hline $\begin{array}{l}\text { Site of stroke, } n(\%) \\
\text { Right hemisphere } \\
\text { Left hemisphere } \\
\text { Brainstem } \\
\text { Cerebellum }\end{array}$ & $\begin{array}{l}116(46) \\
91(36) \\
20(8) \\
4(2)\end{array}$ & & \\
\hline Time since onset, days & $102(64)$ & & \\
\hline $\begin{array}{l}\text { Stroke Impact Scale } \\
\text { Strength (0-100) } \\
\text { Memory (0-100) } \\
\text { Emotion (0-100) } \\
\text { Communication (0-100) } \\
\text { Activities (0-100) } \\
\text { Mobility (0-100) } \\
\text { Affected hand (0-100) } \\
\text { Participation (0-100) } \\
\text { Recovery (0-100) } \\
\text { Physical functioning (0-100) } \\
\text { Total (0-100) }\end{array}$ & $\begin{array}{l}51.80(20.27) \\
81.83(17.48) \\
82.56(13.57) \\
84.89(18.94) \\
70.13(15.44) \\
79.32(14.11) \\
45.10(35.59) \\
66.75(20.84) \\
56.53(16.53) \\
60.74(16.16) \\
70.30(11.54)\end{array}$ & $\begin{array}{l}59.61(22.82) \\
87.08(15.92) \\
81.89(14.51) \\
86.51(18.01) \\
77.43(16.57) \\
85.56(12.90) \\
55.68(37.69) \\
71.94(19.59) \\
63.56(17.34) \\
69.57(19.36) \\
75.61(13.78)\end{array}$ & $\begin{array}{l}<.01 \\
<.01 \\
.49 \\
.04 \\
<.01 \\
<.01 \\
<.01 \\
<.01 \\
<.01 \\
<.01 \\
<.01\end{array}$ \\
\hline Fatigue Severity Scale $(1-7)^{\mathrm{b}}$ & $3.98(1.69)$ & $4.03(1.67)$ & .43 \\
\hline $\begin{array}{l}\text { Hospital Anxiety and Depressio } \\
\text { Depression (0-21) } \\
\text { Anxiety (0-21) }\end{array}$ & $\begin{array}{l}4.40(3.23) \\
3.63(3.27)\end{array}$ & $\begin{array}{l}4.28(4.00) \\
3.66(3.55)\end{array}$ & $\begin{array}{l}.53 \\
.64\end{array}$ \\
\hline $\begin{array}{l}\text { Nottingham Extended ADL } \\
\text { Mobility }(0-18) \\
\text { Kitchen }(0-15) \\
\text { Domestic }(0-15) \\
\text { Leisure }(0-18)\end{array}$ & $\begin{array}{l}10.90(4.21) \\
10.06(3.80) \\
4.53(3.93) \\
7.31(2.87)\end{array}$ & $\begin{array}{l}13.62(4.14) \\
12.45(3.53) \\
7.95(4.08) \\
10.39(3.60)\end{array}$ & $\begin{array}{l}<.01 \\
<.01 \\
<.01 \\
<.01\end{array}$ \\
\hline $\begin{array}{c}\text { Motricity Indexa } \\
\text { Arm (0-100) } \\
\text { Leg }(0-100)\end{array}$ & $\begin{array}{l}60.37(26.41) \\
68.13(20.16)\end{array}$ & $\begin{array}{l}65.35(26.27) \\
72.92(20.41)\end{array}$ & $\begin{array}{l}<.01 \\
<.01\end{array}$ \\
\hline
\end{tabular}

Values are displayed as mean (SD) unless otherwise indicated; * Follow-up was 12 weeks after the baseline assessment, $\mathrm{n}=242 ;{ }^{\text {a }}$ Higher mean scores reflect better function; ${ }^{b}$ Lower mean scores reflect better function. 
For HRQOL (SIS), the mean score at baseline was 70.30 (SD 11.54) and three months later the mean was 75.61 (SD 13.78). The highest score at baseline was 94. One patient reached the maximum score of 100 at the follow-up assessment. Change-from-baseline scores show that 57 patients (24\%) had a negative change score (decline), and the remaining patients had a neutral (change score of $0 ; 3 \%$ ) or positive change score (improvement; $73 \%$ ). The lowest and highest change-from-baseline scores were -23 and +40 , respectively.

Mean upper limb strength (MI-arm) was 60.37 (SD 26.41). Eleven patients (4\%) had a baseline score of 0 in comparison to 21 patients (8\%) with the maximum score of 99 at baseline. Six months post-stroke, mean Ml-arm was 65.35 (SD 26.27), and only seven patients (3\%) had a score of 0 while the number of patients reaching the maximum score increased to $40(17 \%)$. Change-from-baseline scores showed that 39 patients (16\%) had a negative change score, and the remaining patients had a neutral (no change) (28\%) or positive change score (56\%). The lowest and highest change-from-baseline scores were -23 and +52 points, respectively.

Upper limb strength and health-related quality of life cross-sectionally in time Linear regression analysis showed that $\mathrm{HRQ}$ oL was statistically significantly related to upper limb strength at baseline, $F(1,248)=165.023, p<.0005$ and upper limb strength accounted for $40 \%$ of the explained variability in HRQoL (Table 4.2). At follow-up HRQoL was also significantly related to upper limb strength, $F(1,240)=225.191, p<.0005$ and upper limb strength accounted for $48 \%$ of the explained variability in HRQoL (Table 4.2). Table 4.2 also shows that age, sex, side of hemiplegia, MI-leg, HADS and FSS in relation to $\mathrm{HRQ}$ oL at baseline can be included as independent variables in the regression analysis (all $p<.2$ ). At follow-up, age and sex (baseline), MI-leg, HADS and FSS in relation to HRQoL emerged as independent variables (all $p<.2$ ).

Upper limb strength, stroke- and clinical variables, and health-related quality of life over time

HRQoL at baseline (Model 1) was significantly related to HRQoL at follow-up, $\left(R^{2}=.644\right.$, $F(1,229)=414.546, p<.0005)$ (Table 4.3). The addition of (improvement in) upper limb strength to the prediction of HRQoL at follow-up (Model 2) led to a statistically significant increase in $\mathrm{R}^{2}$ of .078, $F(2,227)=31.983, p<.0005$. The addition of MI-leg, FSS, HADS (Model 3) led to small, non-significant changes in $\mathrm{R}^{2}$ (.004) (Table 4.3). The addition of age, sex and hemiplegia (full model) led to a small increase in $\mathrm{R}^{2}$ of .010 , which was statistically significant ( $p<.05$, Table 4.3 for full details). Clinical variables, like leg function (MI-leg), did not show significant correlation with HRQoL $(p>.05)$. Sex was the only stroke-related characteristic with a significant relation with HRQoL $(p<.05)$. 


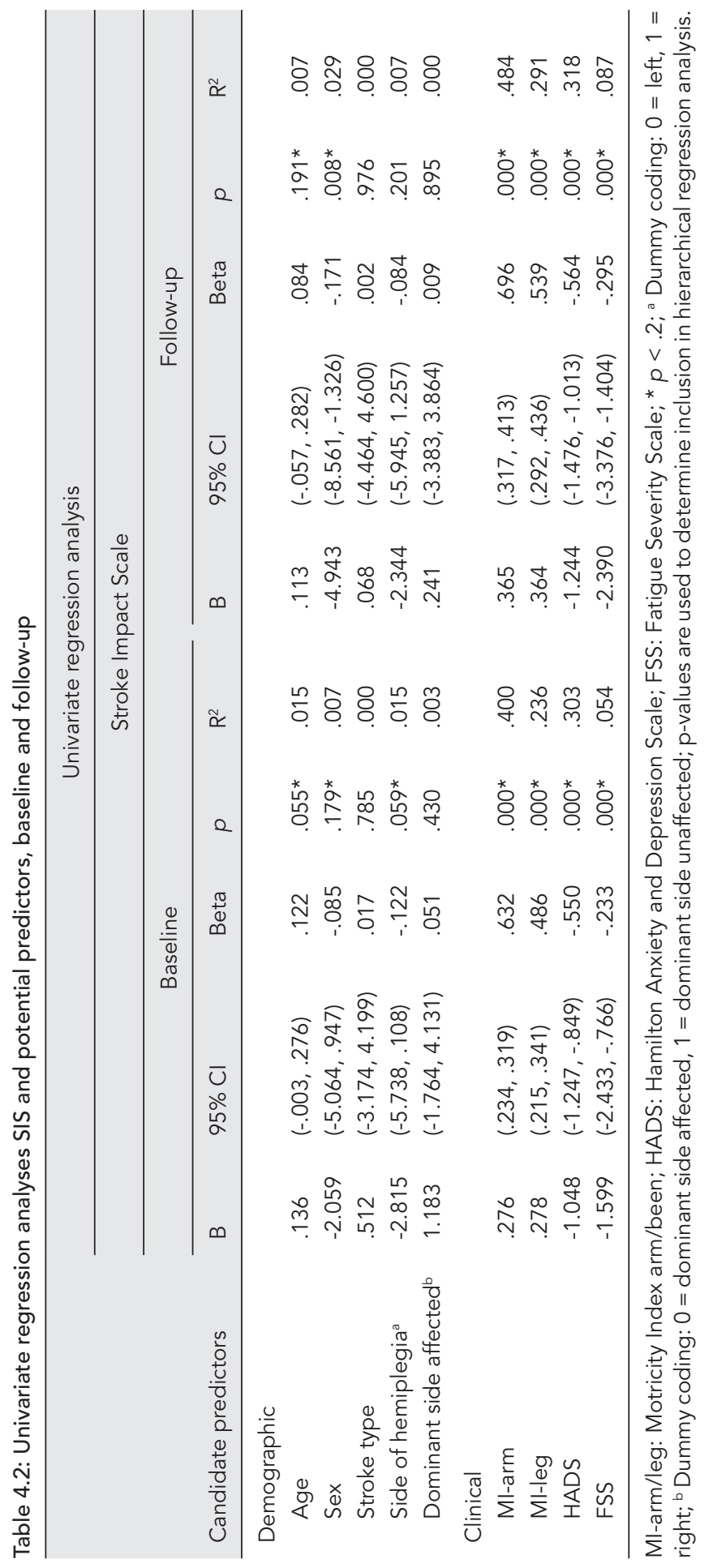


Table 4.3: Hierarchical regression analysis

\begin{tabular}{|c|c|c|c|c|c|c|c|c|}
\hline \multirow[b]{2}{*}{ Baseline } & \multicolumn{8}{|c|}{ Stroke Impact Scale, at follow-up } \\
\hline & B & SE B & $\beta$ & $p$ & $\mathrm{R}^{2}$ & $\mathrm{R}^{2}$ change & $F$ & $p$ \\
\hline Model 1 & & & & & .644 & & 414.546 & $.000^{*}$ \\
\hline SIS total & .965 & .047 & .803 & $.000^{\star}$ & & & & \\
\hline Model 2 & & & & & .722 & .078 & 31.983 & $.000^{*}$ \\
\hline SIS total & .745 & .054 & .620 & $.000^{\star}$ & & & & \\
\hline Ml-arm & .159 & .024 & .302 & $.000^{\star}$ & & & & \\
\hline $\begin{array}{l}\text { Ml-arm (baseline- } \\
\text { follow-up) }\end{array}$ & .290 & .049 & .211 & $.000^{\star}$ & & & & \\
\hline Model 3 & & & & & .726 & .004 & 1.040 & .376 \\
\hline SIS total & .699 & .068 & .582 & $.000^{\star}$ & & & & \\
\hline Ml-arm & .145 & .030 & .274 & $.000^{\star}$ & & & & \\
\hline $\begin{array}{l}\text { Ml-arm (baseline- } \\
\text { follow-up) }\end{array}$ & .283 & .050 & .206 & $.000^{\star}$ & & & & \\
\hline MI-leg & .042 & .033 & .060 & .206 & & & & \\
\hline FSS & .231 & .331 & .028 & .484 & & & & \\
\hline HADS & -.131 & .107 & -.057 & .223 & & & & \\
\hline Model 4 & & & & & .736 & .010 & 2.807 & $.040^{*}$ \\
\hline SIS total & .675 & .070 & .561 & $.000^{\star}$ & & & & \\
\hline Ml-arm & .152 & .030 & .288 & $.000 *$ & & & & \\
\hline $\begin{array}{l}\text { Ml-arm (baseline } \\
\text { - follow-up) }\end{array}$ & .276 & .049 & .201 & $.000 *$ & & & & \\
\hline MI-leg & .041 & .033 & .060 & .209 & & & & \\
\hline FSS & .166 & .338 & .020 & .624 & & & & \\
\hline HADS & -.128 & .107 & -.056 & .230 & & & & \\
\hline Age & -.051 & .049 & -.038 & .297 & & & & \\
\hline Sex & -2.615 & 1.042 & -.090 & $.013^{*}$ & & & & \\
\hline Hemiplegia ${ }^{a}$ & -1.130 & 1.013 & -.041 & .266 & & & & \\
\hline
\end{tabular}

Ml-arm/leg: Motricity Index arm/been; HADS: Hamilton Anxiety and Depression Scale; FSS: Fatigue Severity Scale; ${ }^{a}$ Dummy coding: $0=$ left, $1=$ right; ${ }^{*} p<.05$.

Changes in upper limb strength and changes in health-related quality of life over time According to the $\mathrm{R}^{2}=0.71$ (Figure 4.1), the change scores in upper limb strength between baseline and follow-up accounted for $7.1 \%$ of the variation in change scores in HRQOL between baseline and follow-up. ${ }^{21}$ Change scores in upper limb strength between baseline and follow-up significantly predicted change scores in HRQoL between baseline and followup $(F(1,240)=18.351, p<.0005)$. 


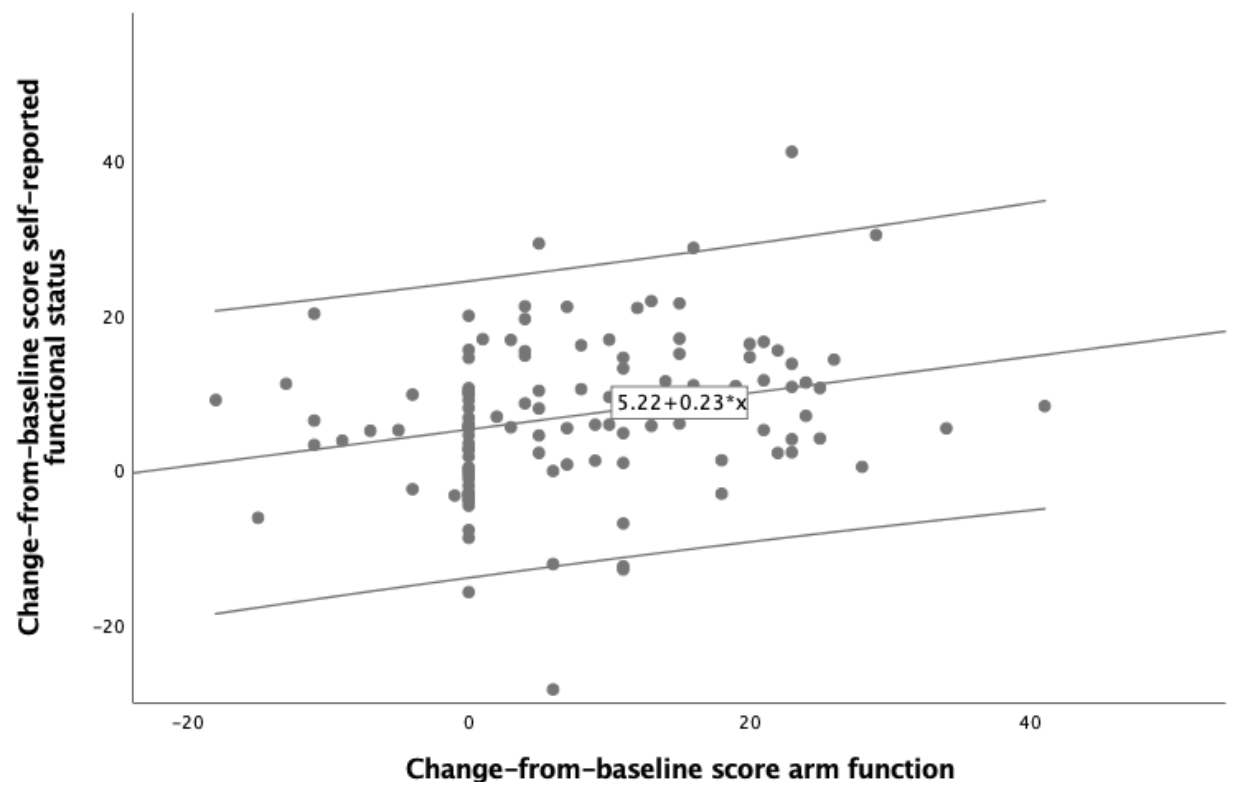

Figure 4.1: Scatterplot and linear regression fit for change-from-baseline scores between baseline and follow-up.

$\mathrm{R}^{2}=.071 ;$ adjusted $\mathrm{R}^{2}=.067 ; F(1,240)=18.351, \mathrm{p}<.0005$.

\section{Discussion}

This is the first study that assesses the degree and relative impact of the relationship, both cross-sectionally and longitudinally, between upper limb strength and HROoL. Upper limb strength was significantly related to HRQoL at baseline and follow-up and is an important significant predictor of HRQoL, even after correcting for clinical and stroke characteristics. Furthermore, an improvement in upper limb strength was positively related to an improvement of HRQoL, which suggests that recovery of upper limb strength is also important during the outpatient rehabilitation period and beyond.

Our finding that upper limb strength is independently related to HRQoL is in line with findings from other studies. ${ }^{14,13,14,22,23}$ In contrast to earlier studies, however, our study is able to demonstrate the relation between upper limb strength and HRQOL in the sub-acute outpatient rehabilitation phase. In addition, patients were enrolled at a specific moment during stroke rehabilitation, namely upon discharge from inpatient rehabilitation. We considered it useful to explore this sub-acute phase, since it is characterized by minimal rehabilitation support and is beyond the sensitive time-window of recovery. ${ }^{24,25}$ Most earlier studies assessed patients within a broad time frame after stroke (e.g. 2-68 months after stroke onset), and took place in the chronic phase. $4,13,14,22,23$ 
Our study highlights the unique contribution of upper limb strength to HRQoL and its importance compared to other predictors. In previous studies arm function was included as one of the many potential predictors of HRQoL and can therefore have been easily overlooked, as these studies did not focus solely on arm function. 1,4,13,14,22 Some previous studies did show that specific domains of HRQoL, i.e. ADL and participation, were related to arm function, ${ }^{13,14}$ and that autonomy in daily life activities was strictly related to recovery of the affected arm. ${ }^{26}$ The current study also demonstrates that upper limb strength contributes more to HRQoL than other predictors, like leg function, which is consistent with the findings of a comparable study, ${ }^{4}$ indicating that upper limb strength is the strongest predictor of HRQoL. It should be noted that this has only been demonstrated in mildly to moderately impaired stroke patients, because the inclusion criteria were limited to this group of patients. ${ }^{4}$

In addition to the importance of upper limb strength, there are several factors that predict HRQoL in stroke patients. Our study demonstrated that HRQoL was also cross-sectionally predicted by age, sex, side of hemiplegia, anxiety and depression, leg function and fatigue. In accordance with most studies, symptoms of anxiety and depression and post-stroke fatigue could affect a patient's motivation to participate in rehabilitation programs and influence (the rate) of recovery. 1,13,27 Older age is associated with poorer HRQoL in stroke patients as in the general population. The association between (female) gender and poorer HRQoL might be explained by a higher prevalence of anxiety post-stroke. ${ }^{1}$ Dominant side of hemiplegia has been repeatedly shown to be associated with HRQoL. Most patients are right-handed with a left hemisphere stroke. Since the left hemisphere controls speech and language function may affect HRQoL through an altered communication ability. 1,28

In contrast to the current study, previous studies have not assessed the effect of changes in upper limb strength over time on HRQoL, as HRQoL was assessed at a single timepoint. 1,4,23,29 The current study shows that improvements in upper limb strength are positively related to improvements in HRQoL during outpatient rehabilitation. This suggests that there should also be a focus on upper limb strength recovery in the outpatient rehabilitation phase, in order to possibly expect improvement in HRQoL. The improvement in upper limb strength found during outpatient rehabilitation raises the possibility that there is potential to train outside the sensitive time-window (i.e. 0-3 months post-stroke). ${ }^{30,31} \mathrm{~A}$ recent study that investigated an intensive upper limb neurorehabilitation programme in chronic stroke patients, found large clinical improvements in measures of impairment and activity. ${ }^{32}$ However, future studies are recommended to confirm the results and unanswered questions about potential for arm recovery beyond the sensitive time-window. Currently, patients receive little to no rehabilitation support when discharged to the community. ${ }^{24,25}$ 
The present study had some limitations. Firstly, included patients were indicated for inpatient rehabilitation when discharged from the hospital and had only mild to moderate stroke, which limits the generalizability of the trial. In addition, the included patients were specifically selected for inclusion in a task-oriented circuit class training because of primary problems in walking competency, what might result in a selection of patients with relatively mild upper limb impairment. Nonetheless, only a few patients reached a maximum score on the upper limb clinical measures at baseline. Secondly, we chose to limit this study to upper limb strength, while sensory function may play a prominent role in a patient's perception of arm function. ${ }^{33}$ Future studies should determine the role of sensory function in the relation between upper limb strength and HRQoL.

Our study provides evidence that upper limb strength is an important independent predictor of HRQoL in the sub-acute phase after stroke. Upper limb strength appears to be the most important predictor of $\mathrm{HRQOL}$, besides other predictors as leg function and anxiety, and an improvement of upper limb strength contributes positively to HRQOL. Current arm function recovery therapies, e.g. constraint-induced movement therapy, ${ }^{34}$ should be optimized and evaluated since improvement in upper limb strength positively influences HRQoL. Future studies on the current topic, and outside the sensitive time-window of recovery, are therefore recommended.

\section{Funding}

This work was supported by the Netherlands Organization for Scientific Research (VICl 016.130.662).

\section{References}

1. Morris, J. H., Van Wijck, F., Joice, S. \& Donaghy, M. Predicting health related quality of life 6 months after stroke: The role of anxiety and upper limb dysfunction. Disabil. Rehabil. 35, 291-299 (2013).

2. Northcott, S., Moss, B., Harrison, K. \& Hilari, K. A systematic review of the impact of stroke on social support and social networks: Associated factors and patterns of change. Clin. Rehabil. 30, 811-831 (2016).

3. Northcott, S. \& Hilari, K. Why do people lose their friends after a stroke? Int. J. Lang. Commun. Disord. 46, 524-534 (2011).

4. Franceschini, M., La Porta, F., Agosti, M. \& Massucci, M. Is health-related-quality of life of stroke patients influenced by neurological impairments at one year after stroke? Eur. J. Phys. Rehabil. Med. 46, 389-399 (2010).

5. Langhorne, P., Bernhardt, J. \& Kwakkel, G. Stroke rehabilitation. Lancet 377, 1693-1702 (2011).

6. Broeks, J. G., Lankhorst, G. J., Rumping, K. \& Prevo, A. J. The long-term outcome of arm function after stroke: results of a follow-up study. Disabil. Rehabil. 21, 357-364 (1999). 
7. Kwakkel, G., Kollen, B. J., Van der Grond, J. V. \& Prevo, A. J. H. Probability of regaining dexterity in the flaccid upper limb: Impact of severity of paresis and time since onset in acute stroke. Stroke 34, 2181-2186 (2003).

8. Persson, H., Parziali, M., Danielsson, A. \& Sunnerhagen, K. Arm function within 72 hours after first occasion of stroke and stroke outcome in an unselected population. Neurorehabil. Neural Repair 26 (6), 732 (2012).

9. Nijland, R. H. M., Van Wegen, E. E. H., Harmeling-Van Der Wel, B. C. \& Kwakkel, G. Presence of finger extension and shoulder abduction within 72 hours after stroke predicts functional recovery: Early prediction of functional outcome after stroke: The EPOS cohort study. Stroke 41, 745-750 (2010).

10. Ekstrand, E., Rylander, L., Lexell, J. \& Brogårdh, C. Perceived ability to perform daily hand activities after stroke and associated factors: A cross-sectional study. BMC Neurol. 16, 1-9 (2016).

11. Waddell, K. J., Birkenmeier, R. L., Bland, M. D. \& Lang, C. E. An exploratory analysis of the selfreported goals of individuals with chronic upper-extremity paresis following stroke. Disabil. Rehabil. 38, 853-857 (2016).

12. Harper, A. et al. Development of the World Health Organization WHOQOL-BREF Quality of Life Assessment. Psychol. Med. (1998). doi:10.1017/S0033291798006667

13. Chen, C.-M. et al. Potential predictors for health-related quality of life in stroke patients undergoing inpatient rehabilitation. Health Qual. Life Outcomes 13, 118 (2015).

14. Nichols-Larsen, D. S., Clark, P. C., Zeringue, A., Greenspan, A. \& Blanton, S. Factors influencing stroke survivors' quality of life during subacute recovery. Stroke 36, 1480-1484 (2005).

15. van de Port, I. G. L. et al. Cost-effectiveness of a structured progressive task-oriented circuit class training programme to enhance walking competency after stroke: the protocol of the FIT-Stroke trial. BMC Neurol. 9, 43 (2009).

16. von Elm, E. et al. The Strengthening the Reporting of Observational Studies in Epidemiology (STROBE) statement: guidelines for reporting observational studies. J. Clin. Epidemiol. (2008). doi:10.1016/j.jclinepi.2007.11.008

17. Vellone, E. et al. Psychometric evaluation of the stroke impact scale 3.0. J. Cardiovasc. Nurs. (2015). doi:10.1097/JCN.0000000000000145

18. Collin, C. \& Wade, D. Assessing motor impairment after stroke: A pilot reliability study. J. Neurol. Neurosurg. Psychiatry 53, 576-579 (1990).

19. Spinhoven, P. et al. A validation study of the Hospital Anxiety and Depression Scale (HADS) in different groups of Dutch subjects. Psychol. Med. 27, 363-370 (1997).

20. Learmonth, Y. C. et al. Psychometric properties of the Fatigue Severity Scale and the Modified Fatigue Impact Scale. J. Neurol. Sci. 331, 102-107 (2013).

21. Cohen, J. Statistical power analysis for the behavioral sciences. Statistical Power Analysis for the Behavioral Sciences 2nd, 567 (1988).

22. Lee, J., Chang, T., Yang, S., Huang, C. \& Hsieh, F. Prediction of quality of life after stroke rehabilitation. Neuropsychiatry (London) 6, 369-375 (2016).

23. Chang, W. H. et al. Predictors of functional level and quality of life at 6 months after a first-ever stroke: the KOSCO study. J. Neurol. 263, 1166-1177 (2016).

24. Meyer, M., Foley, N., Pereira, S., Salter, K. \& Teasell, R. Organized stroke rehabilitation in Canada: Redefining our objectives. Top. Stroke Rehabil. 19, 149-157 (2012).

25. Duncan Millar, J., van Wijck, F., Pollock, A. \& Ali, M. Outcome measures in post-stroke arm rehabilitation trials: do existing measures capture outcomes that are important to stroke survivors, carers, and clinicians? Clin. Rehabil. 33, 737-749 (2019).

26. Smania, N. et al. Active Finger Extension A Simple Movement Predicting Recovery of Arm Function in Patients With Acute Stroke. Eur. J. Phys 45, 349-354 (2009). 
27. Chen, Y. K. et al. Poststroke fatigue: Risk factors and its effect on functional status and health-related quality of life. Int. J. Stroke 10, 506-512 (2015).

28. Foerch, C. et al. Difference in recognition of right and left hemispheric stroke. Lancet (2005). doi:10.1016/S0140-6736(05)67024-9

29. Hartman-Maier, A., Soroker, N., Ring, H. \& Katz, N. Awareness of deficits in stroke rehabilitation. J. Rehabil. Med. 34, 158-164 (2002).

30. Zeiler, S. R. \& Krakauer, J. W. The interaction between training and plasticity in the poststroke brain. Curr. Opin. Neurol. 26, 609-616 (2013).

31. McDonnell, M. N. et al. An investigation of cortical neuroplasticity following stroke in adults: is there evidence for a critical window for rehabilitation? BMC Neurol. 15, 109 (2015).

32. Ward, N. S., Brander, F. \& Kelly, K. Intensive upper limb neurorehabilitation in chronic stroke: Outcomes from the Queen Square programme. J. Neurol. Neurosurg. Psychiatry 90, 498-506 (2019).

33. Meyer, S. et al. Associations between sensorimotor impairments in the upper limb at 1 week and 6 months after stroke. J. Neurol. Phys. Ther. 40, 186-195 (2016).

34. Corbetta, D., Sirtori, V., Castellini, G., Moja, L. \& Gatti, R. Constraint-induced movement therapy for upper extremities in people with stroke. Cochrane Database Syst. Rev. (2015). doi:10.1002/14651858.CD004433.pub3.www.cochranelibrary.com 


\section{Appendix 4.1 Inclusion flow diagram}

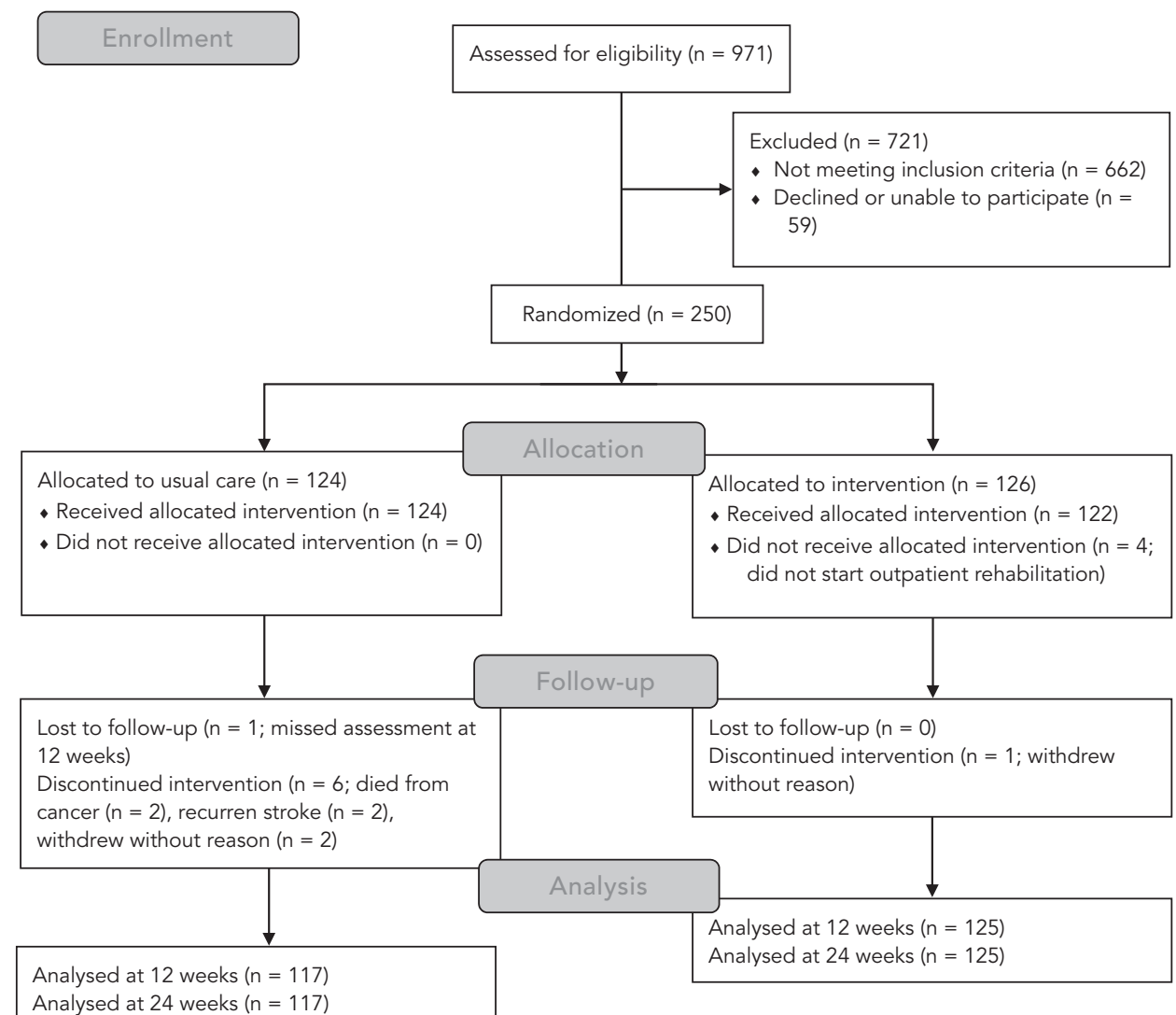




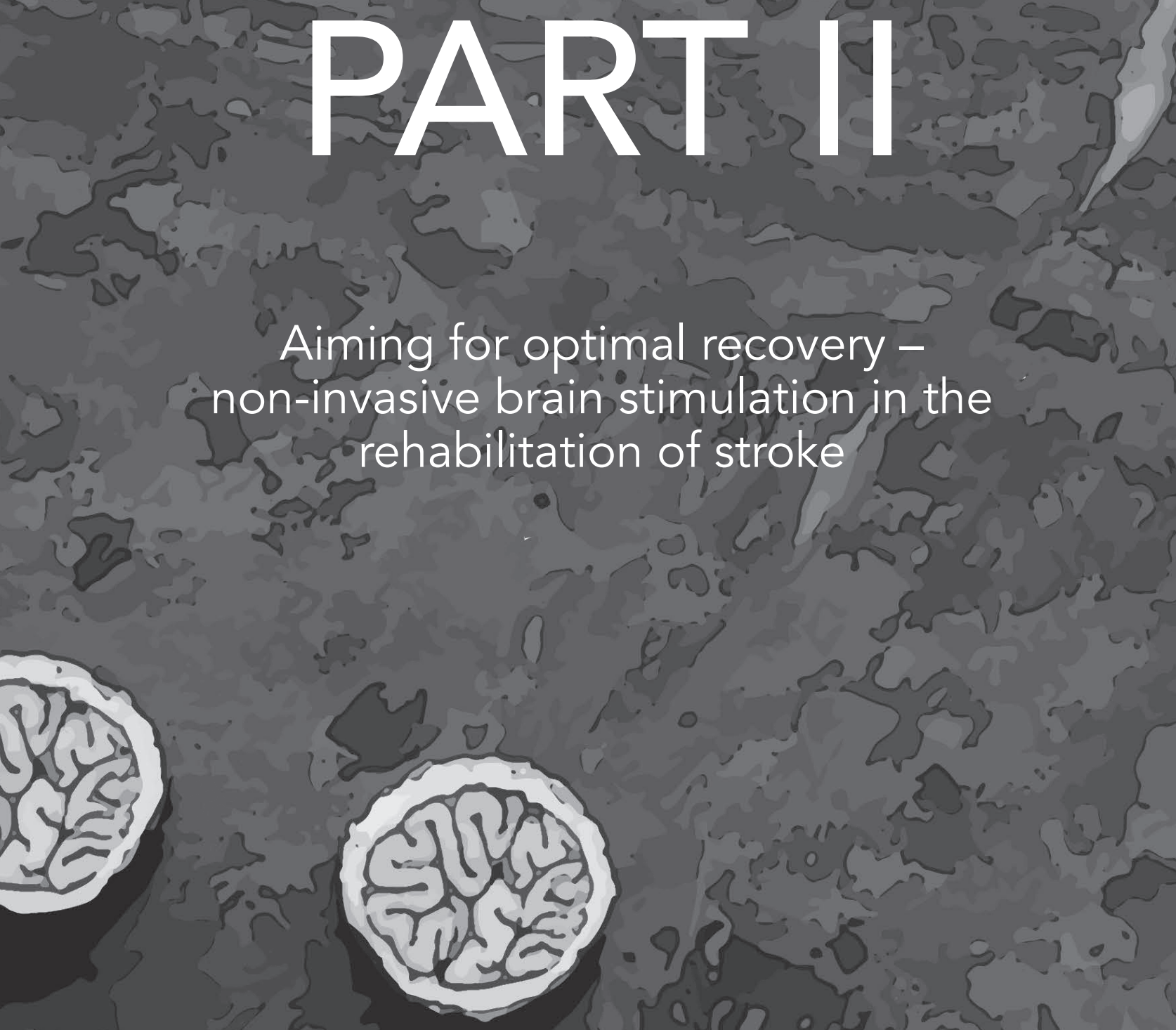

\section{Aiming for optimal recovery - non-invasive brain stimulation in the 18 onrehabilitation of stroke}


ث̊

$\operatorname{lol}^{2}$ renticas

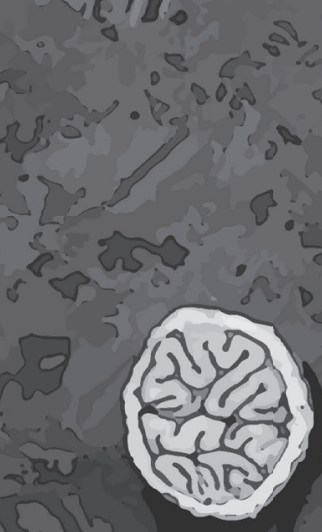

(1) ब्रा

(1)
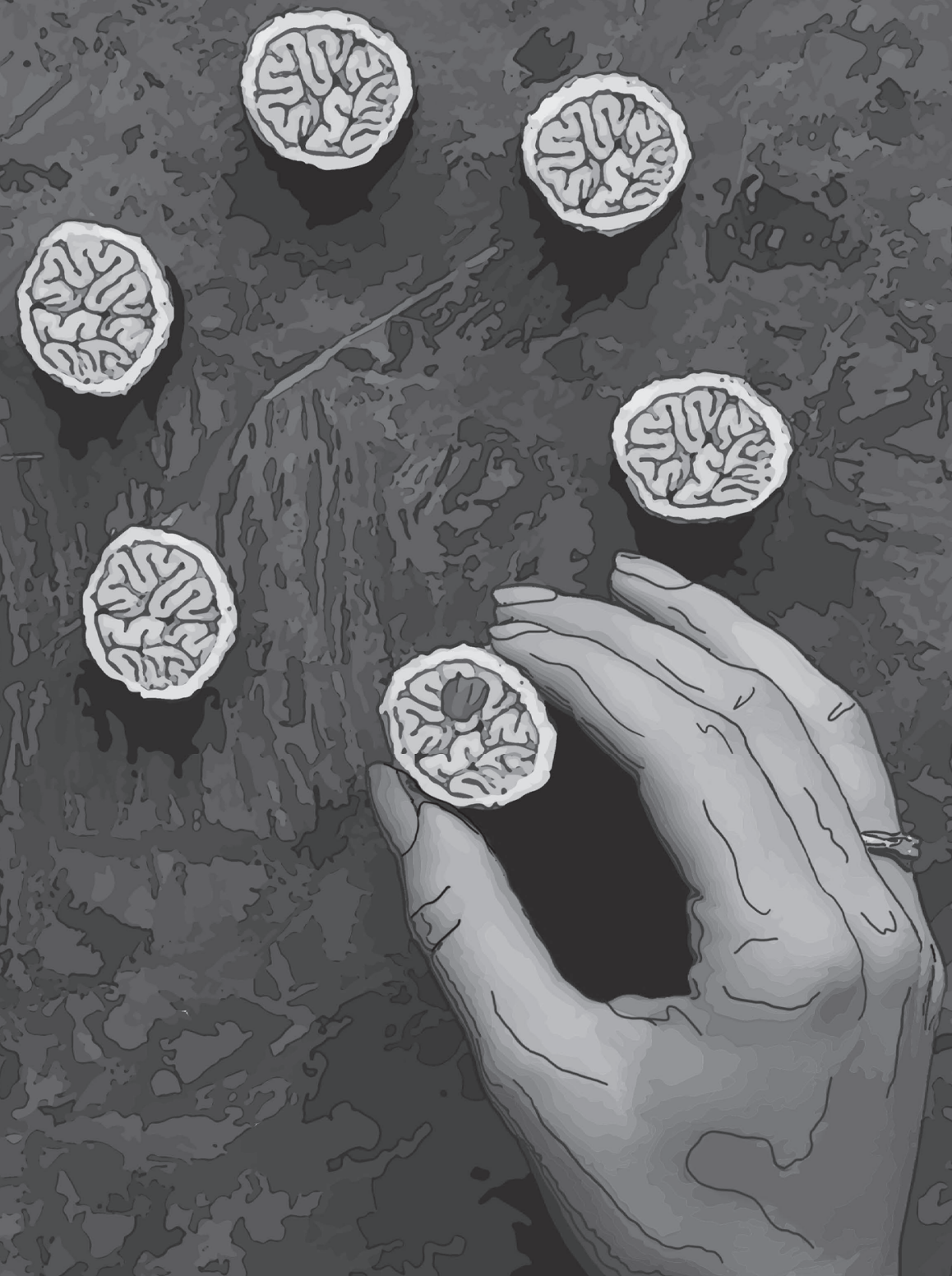


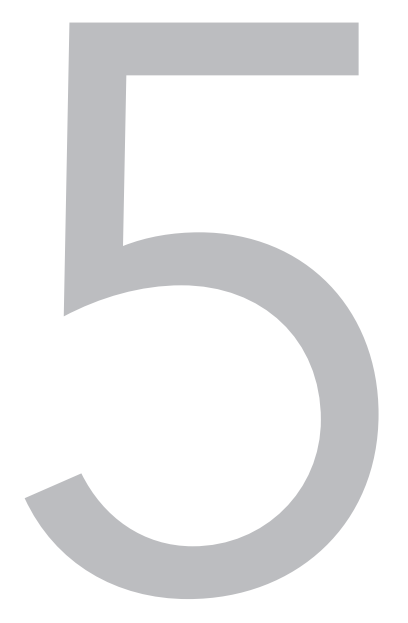

\section{Timing of repetitive transcranial magnetic stimulation onset for upper limb function after stroke: A systematic review and meta-analysis}

Eline C.C. van Lieshout

H. Bart van der Worp Johanna M.A. Visser-Meily Rick M. Dijkhuizen 


\section{Abstract}

Background: Repetitive transcranial magnetic stimulation (rTMS) is a promising intervention to promote upper limb recovery after stroke. We aimed to identify differences in the efficacy of rTMS treatment on upper limb function depending on the onset time post-stroke.

Methods: We searched PubMed, Embase, and the Cochrane Library to identify relevant RCTs from their inception to February 2018. RCTs on the effects of rTMS on upper limb function in adult patients with stroke were included. Study quality and risk of bias were assessed independently by two authors. Meta-analyses were performed for outcomes on individual upper limb outcome measures (function or activity) and for function and activity measures jointly, categorized by timing of treatment initiation. Timing of treatment initiation post-stroke was categorized as follows: acute to early subacute ( $<1$ month), early subacute (1-3 months), late subacute (3-6 months) and chronic ( $>6$ months).

Results: We included 38 studies involving 1,074 stroke patients. Subgroup analysis demonstrated benefit of rTMS applied within the first month post-stroke (MD $=9.31 ; 95 \%$ confidence interval [6.27-12.34]; $p<.0001$ ), but not in the early subacute phase (1-3 months post-stroke) (MD = 1.14; 95\% confidence interval [-5.32-7.59], $p=.73$ ) or chronic phase $\mathrm{s}(>6$ months post-stroke) (MD $=1.79 ; 95 \%$ confidence interval $[-2.00-5.59] ; p=.35)$, when assessed with a function test (Fugl-Meyer Arm test (FMA)). There were no studies within the late subacute phase (3-6 months post-stroke) that used the FMA. Tests at the level of function revealed improved upper limb function after rTMS (SMD $=0.43 ; 95 \%$ confidence interval $[0.02-0.75] ; p=.0001)$, but tests at the level of activity did not, independent of rTMS onset post-stroke (SMD $=0.17 ; 95 \%$ confidence interval $[-0.09-0.44] ; p=.19$ ). Heterogeneities in the results of the individual studies included in the main analyses were large, as suggested by funnel plot asymmetry.

Conclusions: Based on the FMA, rTMS seems more beneficial only when started in the first month post-stroke. Tests at the level of function are likely more sensitive to detect beneficial rTMS effects on upper limb function than tests at the level of activity. However, heterogeneities in treatment designs and outcomes are high. Future rTMS trials should include the FMA and work towards a core set of outcome measures. 


\section{Introduction}

In patients with stroke, paresis of the upper limb is a major cause of disability. ${ }^{1,2}$ This motor disturbance influences activities of daily living, but also the quality of life of patients and their relatives. ${ }^{3,4}$ Neurorehabilitation therefore often focuses on restoration of upper limb function. Several studies have suggested that non-invasive brain stimulation promotes recovery of the upper limb, possibly through enhancement of motor cortex plasticity. ${ }^{5,6}$

Repetitive transcranial magnetic stimulation (rTMS) is a non-invasive, painless method to modulate cortical excitability. High-frequency rTMS or intermittent theta-burst stimulation (TBS) can increase cortical excitability, whereas low-frequency rTMS or continuous TBS can suppress cortical excitability. ${ }^{7}$ Interhemispheric imbalance in primary motor cortex (M1) activity and the remaining functional motor output after stroke may contribute to motor dysfunction and has been suggested as target for therapeutic rTMS. ${ }^{8}$

Earlier meta-analyses of small, randomized controlled trials (RCTs) suggest that rTMS is able to improve motor outcome in the paretic arm after stroke. 9,10 However, there are large differences between the results of RCTs, which could be explained by methodological differences, ${ }^{11,12}$ including the timing of treatment initiation after stroke.

Research to date has not yet determined which time period post-stroke would be the optimal time window to start treatment. Many clinical practice guidelines advocate an early start of rehabilitation after stroke. ${ }^{13}$ Results from studies in animal models and patients suggest that there is an early critical time window during which the brain is most responsive to neurorehabilitation treatments. ${ }^{14}$ Most recovery takes place during the first three months, after which improvement is believed to reach a plateau phase. ${ }^{15,16}$ However, it remains unknown whether rTMS interventions early after stroke could be more effective than at later points in time. Furthermore, the used outcome measure(s) to assess upper limb function must match with the stated intention of the treatment. Outcomes can be measured at the level of function, activity (capacity and performance) or participation, according to the International Classification of Function, Disability and Health (ICF model). ${ }^{17}$ An outcome measure at function level (e.g. Fugl-Meyer Assessment (FMA)) may be more sensitive to effects of interventions targeted at the neural level, than outcome measures at the level of activity or participation (e.g. Action Research Arm Test (ARAT)), which are also affected by cognitive, personal and environmental factors. ${ }^{18}$

We performed a systematic review and meta-analysis to evaluate whether the efficacy of rTMS on upper limb function depends on the time of treatment initiation after stroke. As secondary aims, we also assessed the efficacy of rTMS on upper limb function at the levels of function and activity (ICF model), and determined the efficacy of rTMS applied in the first month post-stroke on upper limb function assessed at three months post-stroke. 


\section{Methods}

This systematic review was conducted according to the Preferred Reporting Items for Systematic Reviews and Meta-Analyses (PRISMA) guidelines. ${ }^{19}$ We did not register the protocol in a registry prior to publishing.

\section{Search strategy and selection of studies}

We searched the literature in three databases (PubMed, Embase, Cochrane Library) for RCTs published up to February 2018 as a full-text article in the English language. We based our search on the following overarching PICO:

In adult patients ( $\geq 18$ years) with stroke (population), does rTMS aimed at improvement of upper limb function (intervention) as compared with sham rTMS or no rTMS (comparison) improve function or activity of the upper limb (outcome)?

We used the key terms 'stroke,' 'transcranial magnetic stimulation,' 'upper limb function,' or their synonyms (for a detailed search strategy, see Appendix 5.1). Manual searches of the reference lists of the selected articles were also conducted.

Studies were excluded if rTMS was part of a coupling/priming protocol or if it was bilateral; if there was no upper limb outcome or stroke severity scale measurement (e.g. NIHSS score) as outcome assessment; or if information required to perform a meta-analysis (e.g. mean scores, standard deviations) was missing. When necessary, authors were contacted, or procedures were deployed for estimation of missing data (see Analyses). Two reviewers (EvL and RC) evaluated the retrieved literature based on titles and abstracts. Differences were discussed until consensus was reached.

\section{Critical appraisal of studies}

The methodological quality and risk of bias of the included studies were evaluated with the PEDro scale (Physiotherapy Evidence Database from the Centre for Evidence-Based Physiotherapy of The George Institute for Global Health). ${ }^{20}$ The eleven items on the scale can be rated as present or absent, with a maximum score of 10 (one item is excluded in the PEDro score). The sum score was classified according to the Canadian Stroke Rehabilitation EvidenceBased Review (SREBR), which categorized the study quality as excellent (9-10), good (6-8), fair (4-5), or poor (0-3). ${ }^{21}$ As a modification, studies scoring 6 or higher in which the critical criteria 2 or 3 (randomization and concealment of allocation, respectively) were absent, were downgraded to fair quality. The methodological quality and risk of bias were rated independently by two reviewers (EvL and $\mathrm{RC}$ ), compared and discussed until consensus was reached. 


\section{Data extraction}

The following data were extracted from the included studies: number of subjects; demographic and clinical characteristics of the subjects (age, gender, time since stroke); intervention protocols (type of rTMS and additional therapies, intensity, number of pulses and sessions, type of coil); outcome measures and mean differences and standard deviations (SDs) of the change scores or means and SDs of the scores after intervention. The extracted data was cross-checked by the second reviewer (RC).

We made an overview of all outcome measures used in the included studies and selected the outcome measures that were used in at least two studies, to enable analysis of results per individual outcome measure. Outcome measures were categorized according to the ICF model to group them at the level of function or activity for further analysis.

We made a categorization of time of treatment post-stroke according to the recent recommendations by the Stroke Recovery and Rehabilitation Roundtable (SRRR) taskforce ${ }^{22}$ : acute to early subacute ( $<1$ month), early subacute (1-3 months), late subacute (3-6 months) and chronic ( $>6$ months). The SRRR categorization of acute (1-7 days) and early subacute (7 days-3 months) were taken together and divided into acute to early subacute $(<1$ month) and early subacute (1-3 months), because most recovery of motor function takes place within the first 30 days post-stroke..$^{23}$ In this way, all included studies could fit within a (specific) treatment timeframe. We checked whether the real and sham rTMS conditions of crossover studies could fit within the specific timeframe.

\section{Data analysis}

Cohen's kappa was calculated to check the interrater reliability of the selection and inclusion of articles.

For quantitative synthesis, effect sizes were calculated based on the change between baseline and post-intervention measurement, or the post-intervention score if the baseline score was not given, in the rTMS and control groups, divided by the pooled standard deviation. We calculated the standard deviation when only t-values and standard error of the mean (SEM) were reported. If there was no numerical data provided, we extracted these from the figures, using Plot Digitizer 2.6.8 based on the Cochrane Handbook for Systematic Reviews of Interventions. ${ }^{24}$ In case of repeated outcome assessments, the first assessment performed after the treatment was used to represent the post-intervention data. Crossover trials were included when point estimates and associated precision of point estimates were given, and when a washout period was incorporated. Standardized mean differences (SMDs), instead of the mean difference (MD), with 95\% confidence intervals (95\% Cls) were used if the outcome measurement scale was not identical between studies. The (unstandardized) 
mean difference was used when change-from-baseline scores were combined with postintervention scores. If a study had multiple treatment groups, the results for the individual treatment conditions were compared. If the results were comparable, an overall effect for the different treatment conditions was computed and used (multiple comparison correction). ${ }^{24}$

To investigate the effect of rTMS treatment in the subacute or chronic phase after stroke, subgroup analyses were performed for the individual outcome measures found. Subgroup analyses of the different timings of post-stroke rTMS treatment were also performed for function and activity outcome measures jointly.

To determine the effect of rTMS applied within the first month post-stroke on upper limb function assessed 3 months post-stroke, an independent analysis was performed.

To determine the potential influence of rTMS frequency (low to the unaffected hemisphere versus high to the affected hemisphere), number of treatment sessions and additional therapy (rTMS alone versus rTMS + therapy), additional subgroup analyses were performed for the function and activity outcome measures (while maintaining the differentiation between subacute and chronic groups). At least two treatment time-points had to be represented in the subgroups, and a subgroup had to consist of at least one study.

The heterogeneity of the effect sizes was assessed with Cochran's Q-test and the inconsistency $\mathrm{I}^{2}$ index, in order to assess the consistency between the trials. ${ }^{24}$ The heterogeneity of the outcome measurements determined the use of a fixed or random effects method. When $I^{2}$ was $>50 \%$, indicative of substantial heterogeneity, and the $p$-value from the Chi-squared test was below 0.05 , a random effects model was applied. The weight of each study, for its effect on the pooled result, was determined by the sample size and confidence interval. The effect sizes were classified as small $(<0.2)$, medium $(0.2-0.8)$, or large $(>0.8) .{ }^{25}$

A funnel plot was used to assess publication bias. Sensitivity analyses were conducted by omitting low quality studies (single-blind studies and without concealed treatment allocation) and studies with a crossover design to determine their influence on the effect size.

Analyses were performed with Review Manager, version 5.3. ${ }^{26}$

\section{Results}

Of 1,737 articles identified in the electronic database search, 38 were included in the systematic review, involving a total of 1,074 subjects. The interrater reliability, measured by Cohen's kappa, was 0.86 , demonstrating almost perfect agreement. ${ }^{27}$ Figure 5.1 shows a flow diagram of the selection process. 


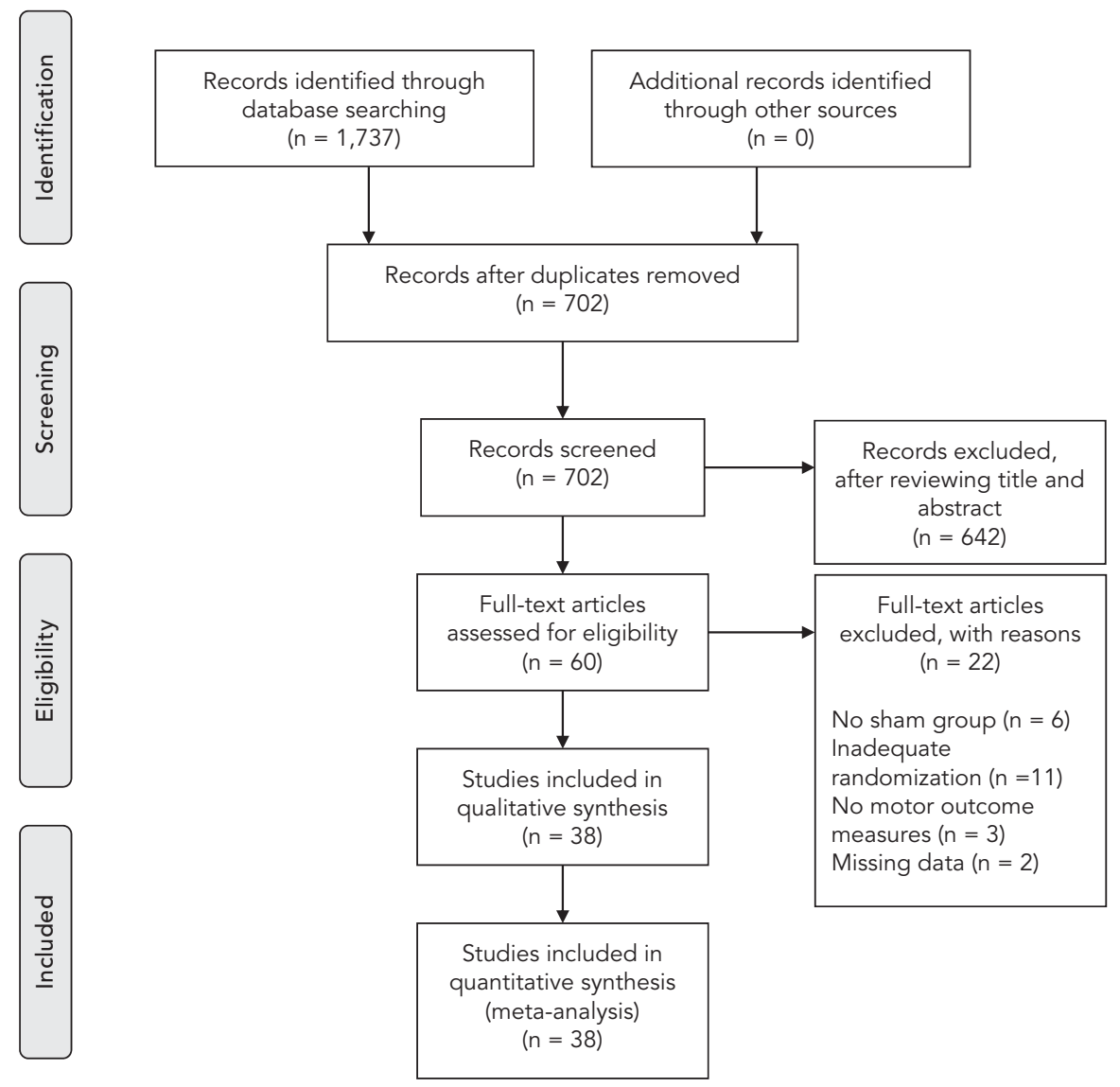

Figure 5.1: PRISMA flow chart.

\section{Characteristics of the studies}

Study characteristics are described in Table 5.1. All studies were designed as RCTs, and six of these studies were designed as randomized controlled crossover studies. ${ }^{28-33}$ The real and sham rTMS conditions of the crossover studies took place within the specific timeframes (i.e. $<1$ month, 1-3 months, 3-6 months, $>6$ months post-stroke) and the wash-out periods ranged from 30 minutes to $>1$ week. The mean patient age in the studies ranged from $46^{28}$ to $75^{34}$ years. Nineteen studies $29,30,33,35-51$ included patients more than 6 months after stroke onset; six $x^{28,52-56}$ between 3 and 6 months after stroke, and twelve within 1 month after stroke. ${ }^{31,32,34,57-64}$ The time between stroke onset and start of treatment varied from 6 days $s^{57}$ to 4 years. ${ }^{39}$ Twenty-four studies were funded by grants from universities, governmental agencies, hospitals or medical foundations. For fourteen studies it was either explicitly mentioned that the work was not supported by any grant from the public or private sector or that there was nothing to disclose financially, or information on funding was not available. 


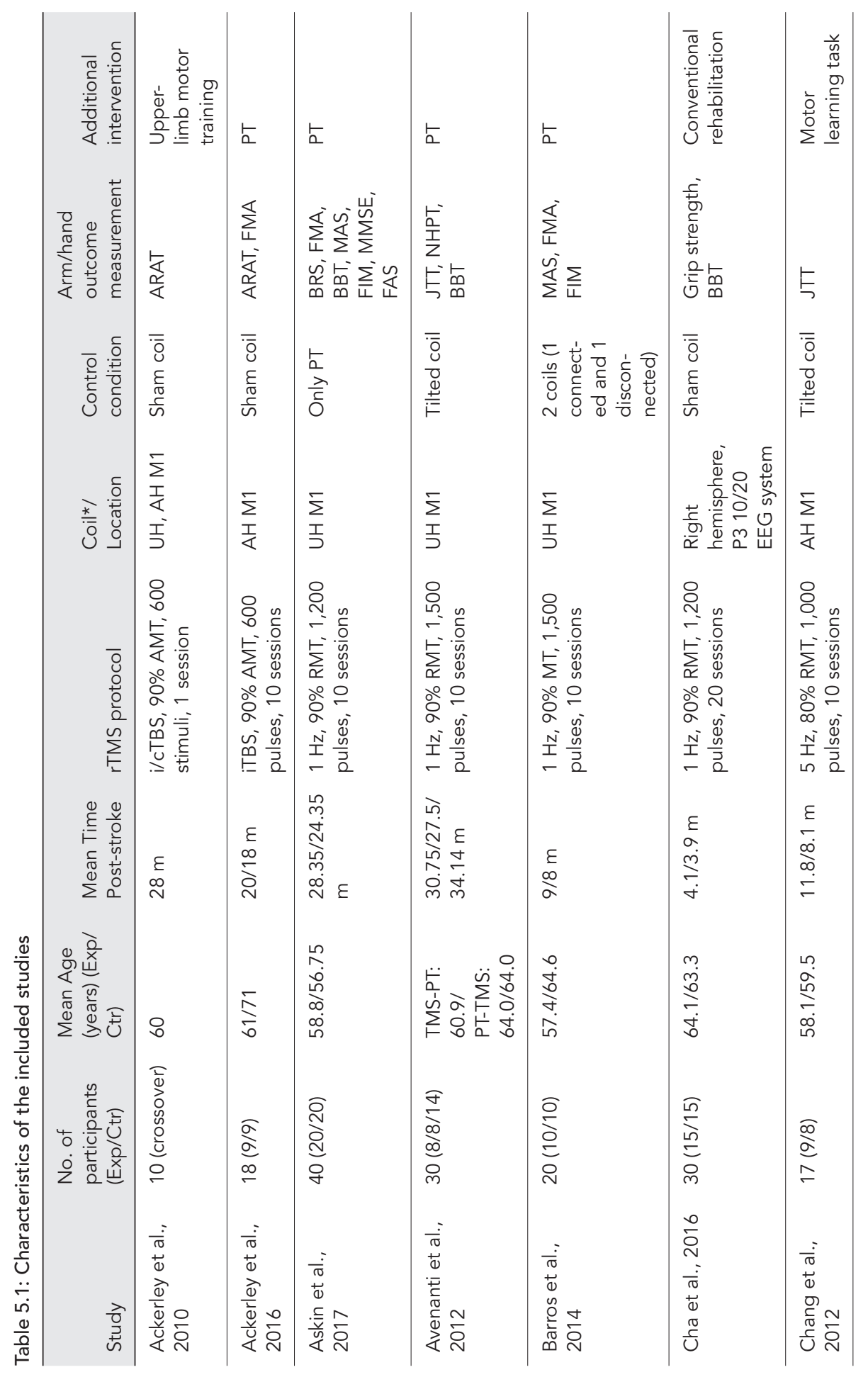




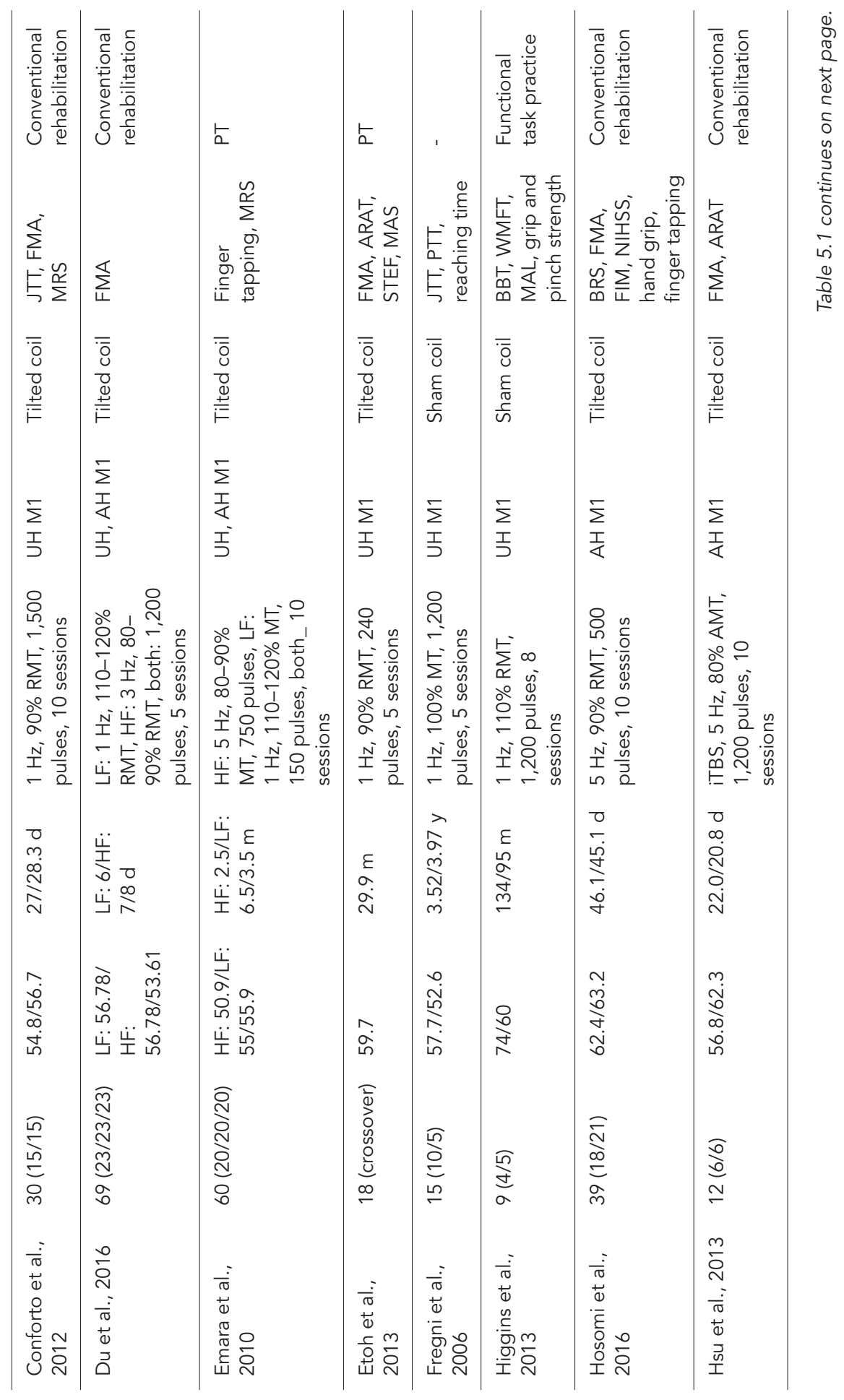




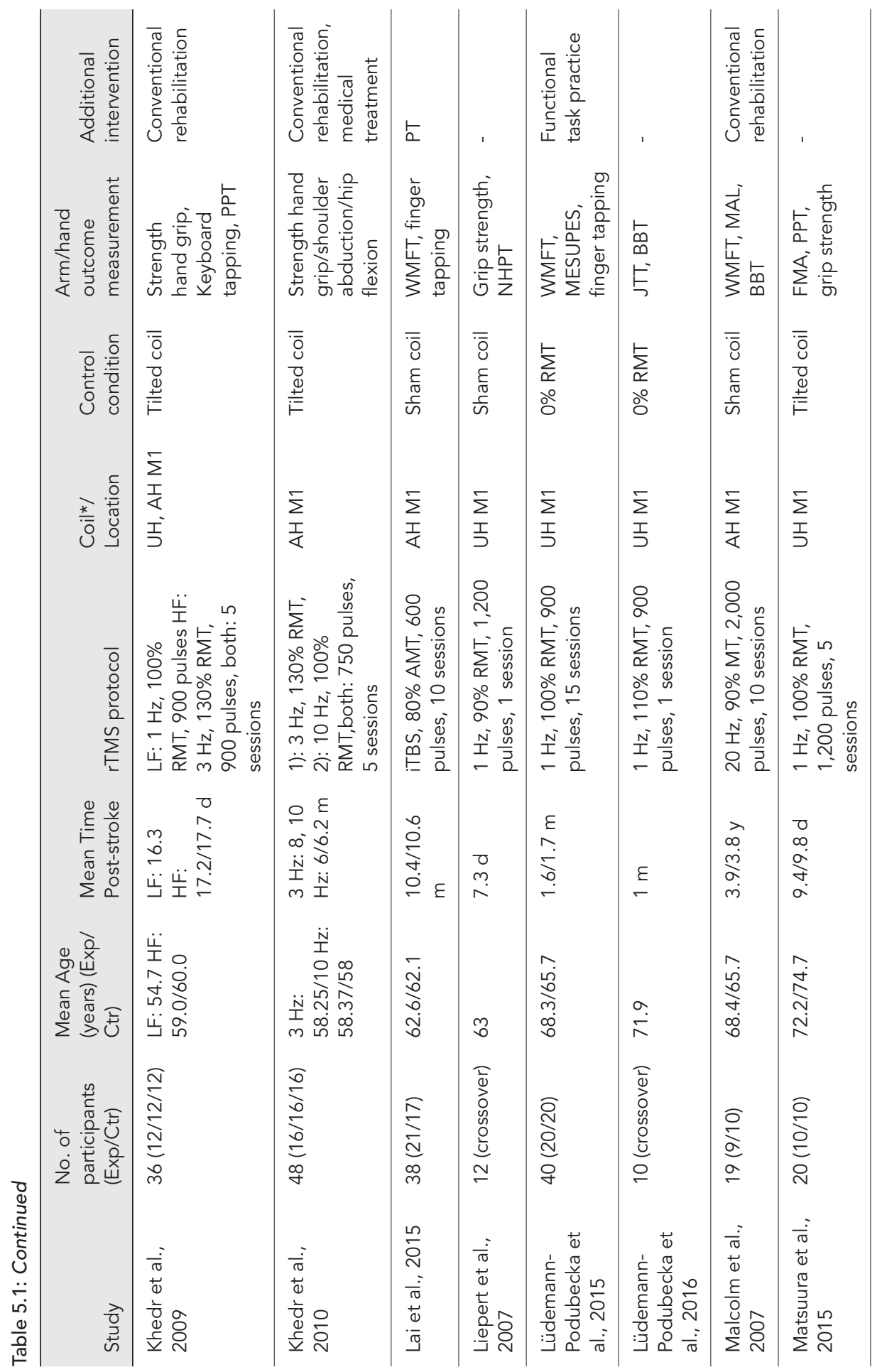




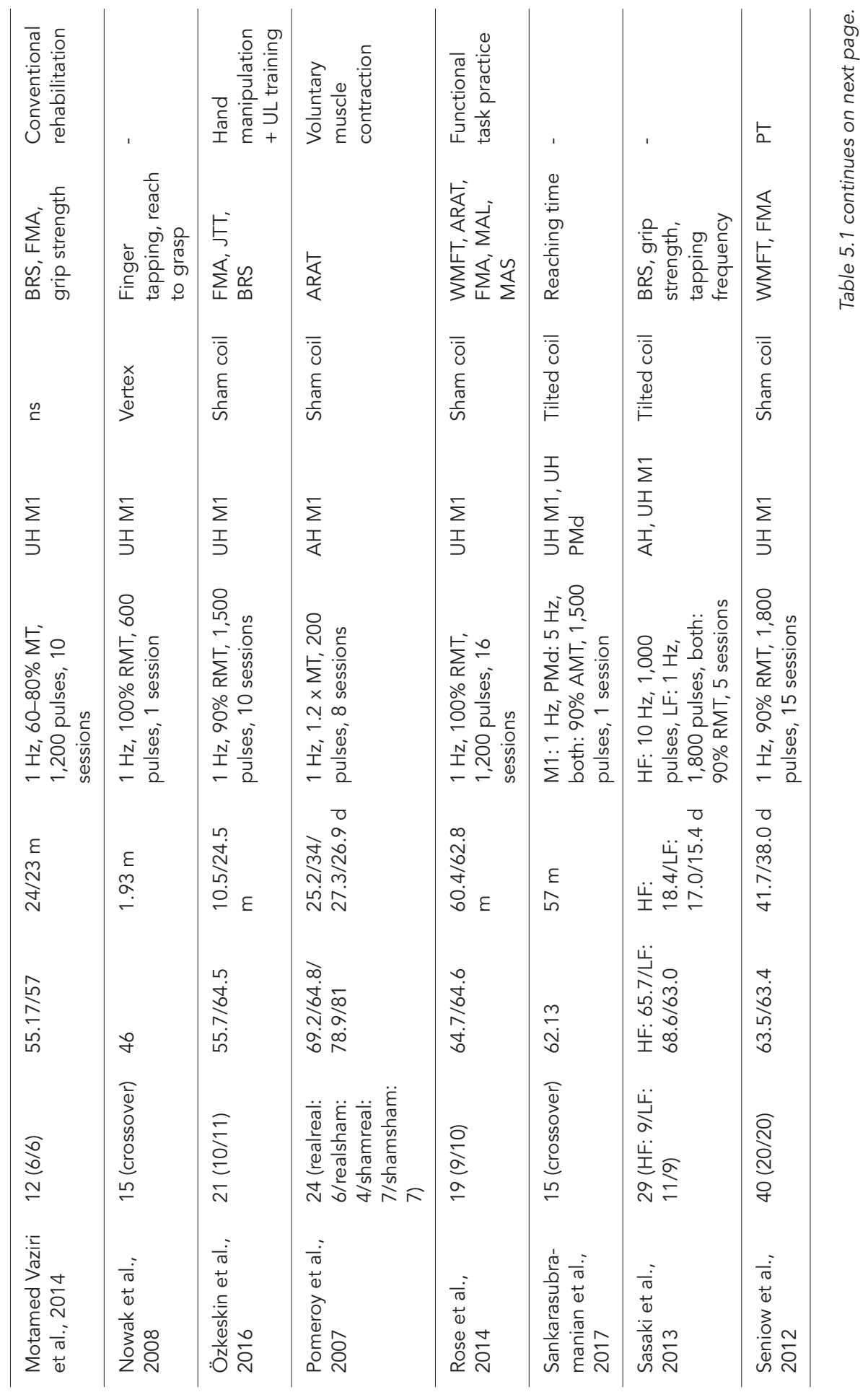




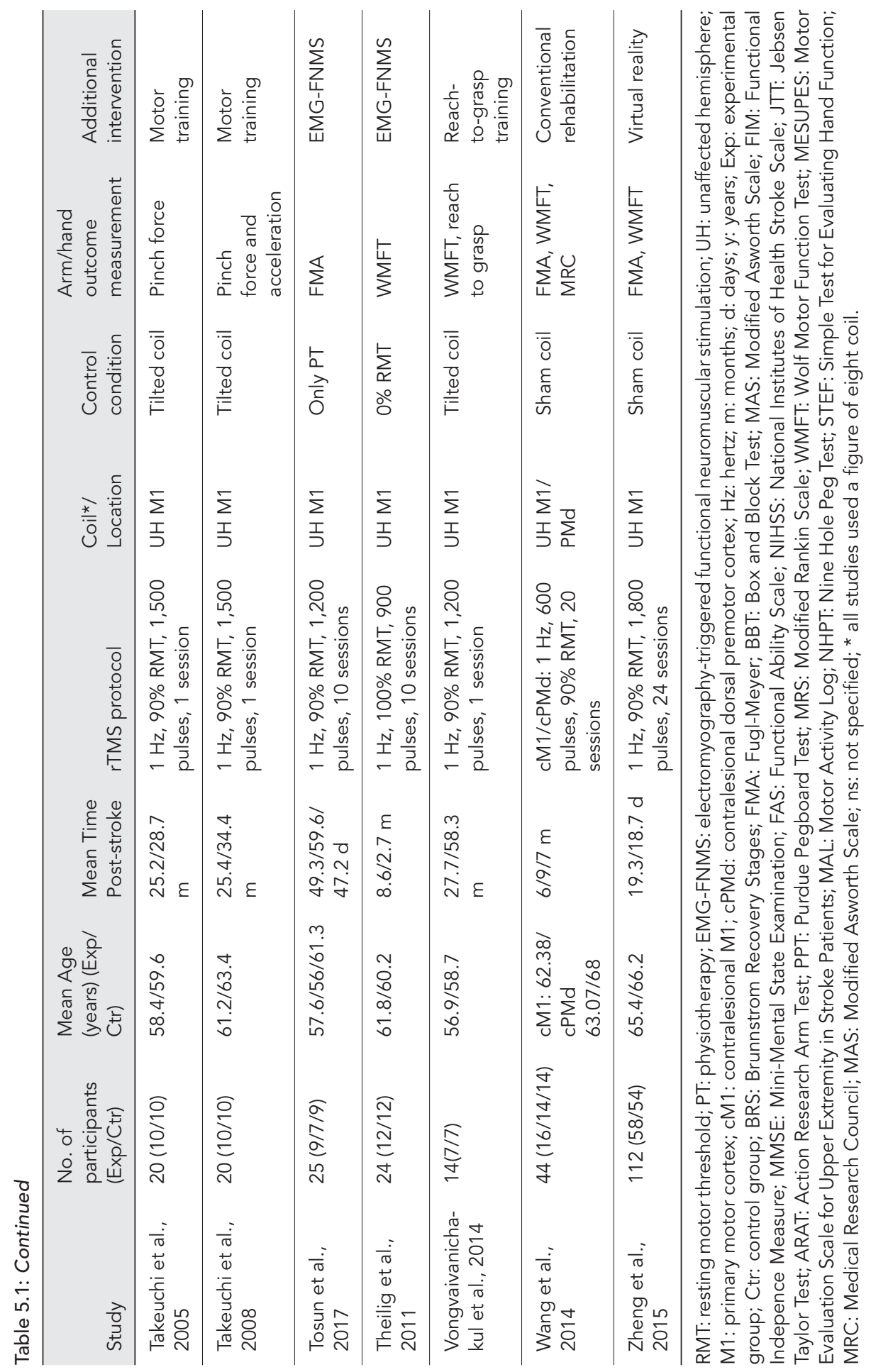




\section{Treatment characteristics}

Different TMS treatment protocols were used in the included studies. In 25 studies, $1 \mathrm{~Hz}$ rTMS was applied to the unaffected hemisphere, with 200-1,800 pulses per session. ${ }^{28,30-32,34,35,37-41,43-45,47,49-51,53,54,56,58,60,61,65}$ Three studies applied high-frequency $r T M S$ to the affected hemisphere with frequencies ranging from $5 \mathrm{~Hz}(500$ and 1,000 pulses) to $20 \mathrm{~Hz}$ (2,000 pulses). ${ }^{36,42,52}$ Intermittent TBS to the affected hemisphere with 600 or 1,200 pulses was applied in three studies. ${ }^{46,48,64}$ Seven studies applied a combination of lowand high-frequency rTMS (ranging from $1 \mathrm{~Hz}$ to $10 \mathrm{~Hz}$ ) or a combination of continuous and intermittent TBS (ranging from 150 to 1,800 pulses) to the unaffected or affected hemispheres respectively (crossover study design, ${ }^{26,30}$ low/high or continous/intermittent group and sham group $32,53,56,59,60)$.

In all studies the primary motor cortex was targeted, of which two studies also targeted the premotor cortex. 29,44 One study was an exception, as only the P3 area (based on a 10/20 EEG system) was targeted. ${ }^{56}$

All studies used a figure-of-eight coil for real rTMS treatment. Sham stimulations were executed with sham coils, tilted coils or real coils without stimulator output, or by vertex stimulation. One study did not describe details of the sham rTMS. ${ }^{41}$ The treatment protocol period ranged from one session $28,29,31-33,38,40,50$ to twenty-four sessions. ${ }^{58}$

Ten different additional therapies were used in combination with the rTMS protocol. The program of the therapy was not always defined, and conventional rehabilitation differed between studies, e.g., conventional rehabilitation could consist of physical therapy and occupational therapy, but could also involve functional task practice or passive limb movement. Conventional rehabilitation (eleven studies) and physical therapy (eight studies) were the most frequently applied additional therapeutic interventions. Virtual reality training, reach to grasp training, a motor learning task and voluntary muscle contraction were used in the other studies. For the studies with an outcome assessment three months post-stroke, it was unclear if patients received the additional therapy (i.e. physical-, conventional- and physiotherapy) also after rTMS. Seven studies did not report or included a therapeutic intervention in addition to rTMS.

\section{Outcome measurements}

The arm/hand motor scales on which outcomes were assessed varied across the studies, and some studies used multiple outcome measures. For meta-analysis, eight different arm/hand motor scales were selected and classified as measures of (body) function: FuglMeyer Arm (FMA), Reaching Time (RT), Grip Strength (GS), Tapping Frequency (TF) and Pinch Force (PF)), or as measures of activity: Jebsen Taylor Test (JTT), Wolf Motor Function 
Test (WMFT) and Action Research Arm Test (ARAT). Measures of (body) function signify measures of motor impairment.

The FMA was the most frequently used test in the included studies ( $n=16$ ), of which five used this as the primary outcome measure. ${ }^{35,41,47,57,66}$ The other outcome measures were less frequently used and a minority of the studies $(n=6)$ included the WMFT or TF (Table 5.1).28,29,38,40,50,51 All studies assessed the scales mentioned above before and after treatment. More than half of the included studies $(n=21)$ had only a single post-intervention measurement. The remaining studies included outcome measurements at multiple timepoints, up to one year ${ }^{63}$ after the intervention.

\section{Methodological quality and risk of bias}

Total scores on the PEDro scale for the included studies ranged from $4^{41}$ to $10,35,48,49,51,53-58$ There were no low quality studies (PEDro score $\leq 3$ ). Eligibility criteria, random allocation, between-group statistical comparisons, point estimates and measures of variability were reported in all studies. Seventeen (45\%) studies did not report if treatment allocation was concealed, and in another seventeen studies the treating therapists were not blinded (Table 5.2).

\section{Meta-analysis}

\section{Subacute versus chronic treatment}

When assessed with the FMA, the benefit of early treatment ( $<1$ month post-stroke) was larger than that of treatment in the early subacute phase (1-3 months) and chronic phase (Figure 5.2). Separate analyses indicated the difference between the different post-stroke phases. The acute to early subacute phase ( $<1$ month) explicitly compared to the early subacute (1-3 months) phase showed a significant subgroup difference in favor of the acute to early subacute phase $(p=.02)$. The acute to early subacute phase also showed a benefit when compared to the chronic phase $(p=.002)$ (Supplementary Figures S5.2.1-S5.2.3 in Appendix 5.2). For the other scales, ICF function and activity measures, the effects of early and late treatment did not differ (Supplementary Figures S5.3.1-S5.3.8 in Appendix 5.3). However, the ICF function measures RT and FT (Supplementary Figures S5.3.1 and S5.3.2 in Appendix 5.3) did show an overall positive effect of rTMS on upper limb function for the early treatment group (FT), which was not observed when treatment was started later. Sensitivity analysis showed minimal impact on the results after removal of the crossover, single-blind and no treatment allocation studies (Appendix 5.4). The funnel plot showed that the estimated treatment effects scattered around the total overall estimate of the meta-analysis (Appendix 5.5). Asymmetry in the funnel plot is noticeable. 


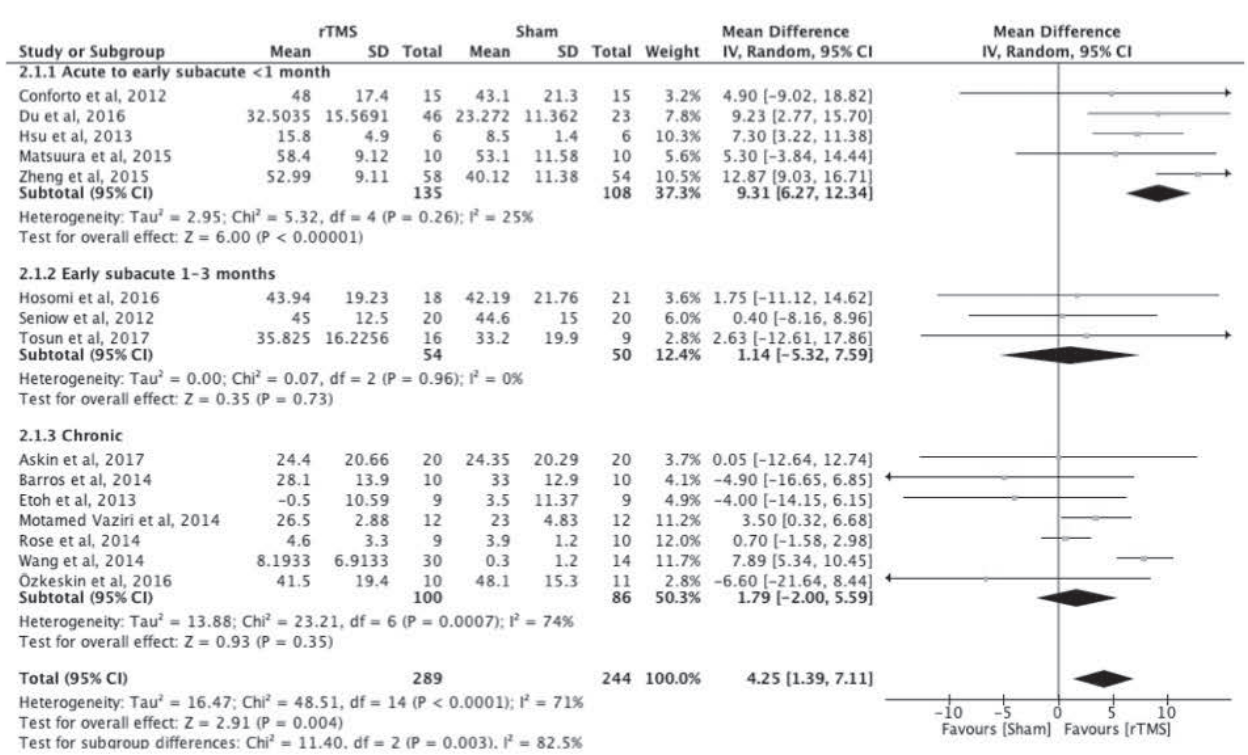

Figure 5.2: Effects of rTMS on the FMA scale, comparing different treatment onset times. Estimates of effect size are shown with $95 \% \mathrm{Cls}$. Final value and change scores combined as mean differences. The mean difference (MD) and 95\% confidence intervals (Cls); No studies within 3-6 months poststroke subgroup.

\section{Function versus activity}

There were no differences between the early and late treatment groups for studies categorized as assessing ICF function (FMA, GS, FT and PF) and activity (JTT, ARAT and WMFT) measures (Figures 5.3-5.4). A benefit of real rTMS was only observed when outcomes were assessed with an ICF function measure (Figure 5.3). Sensitivity analysis showed minimal impact on the results after removal of the crossover, single-blind and no treatment allocation studies (Appendix 5.4).

Treatment within 1 month and outcome at 3 months

rTMS intervention within 1 month after stroke improved upper limb function at 3 months ( $p$ <.0001; Figure 5.5) (ICF function measures: FMA and GS).

\section{Other subgroup analyses}

Subgroup analyses (number of treatment sessions, additional therapy, rTMS frequency/site of stimulation) revealed statistically significant beneficial treatment effects on the ICF function measures, but not on the ICF activity measures (Appendix 5.6). In the analysis in which the number of treatment sessions was divided into different subgroups (1 session, 2-10 sessions, 


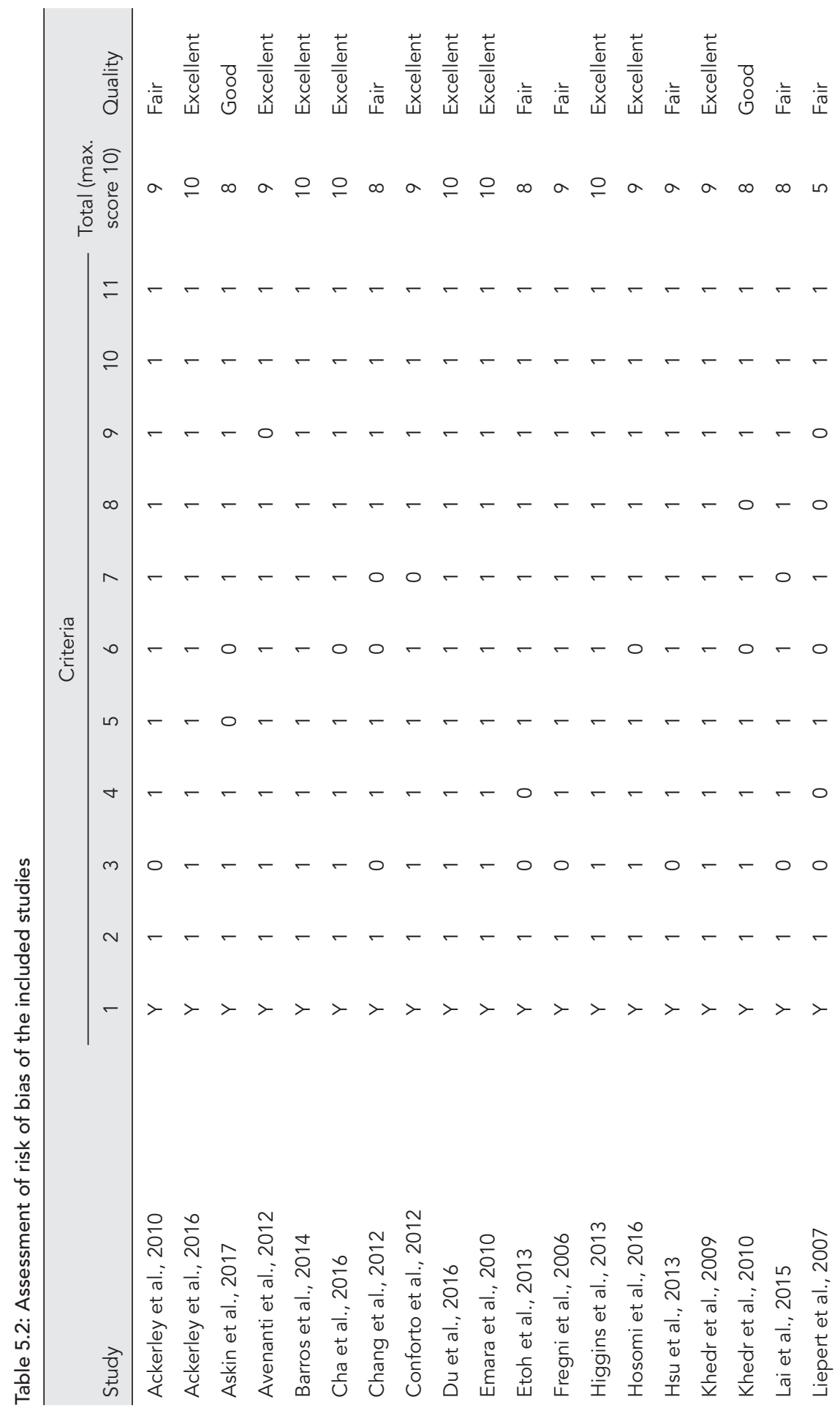




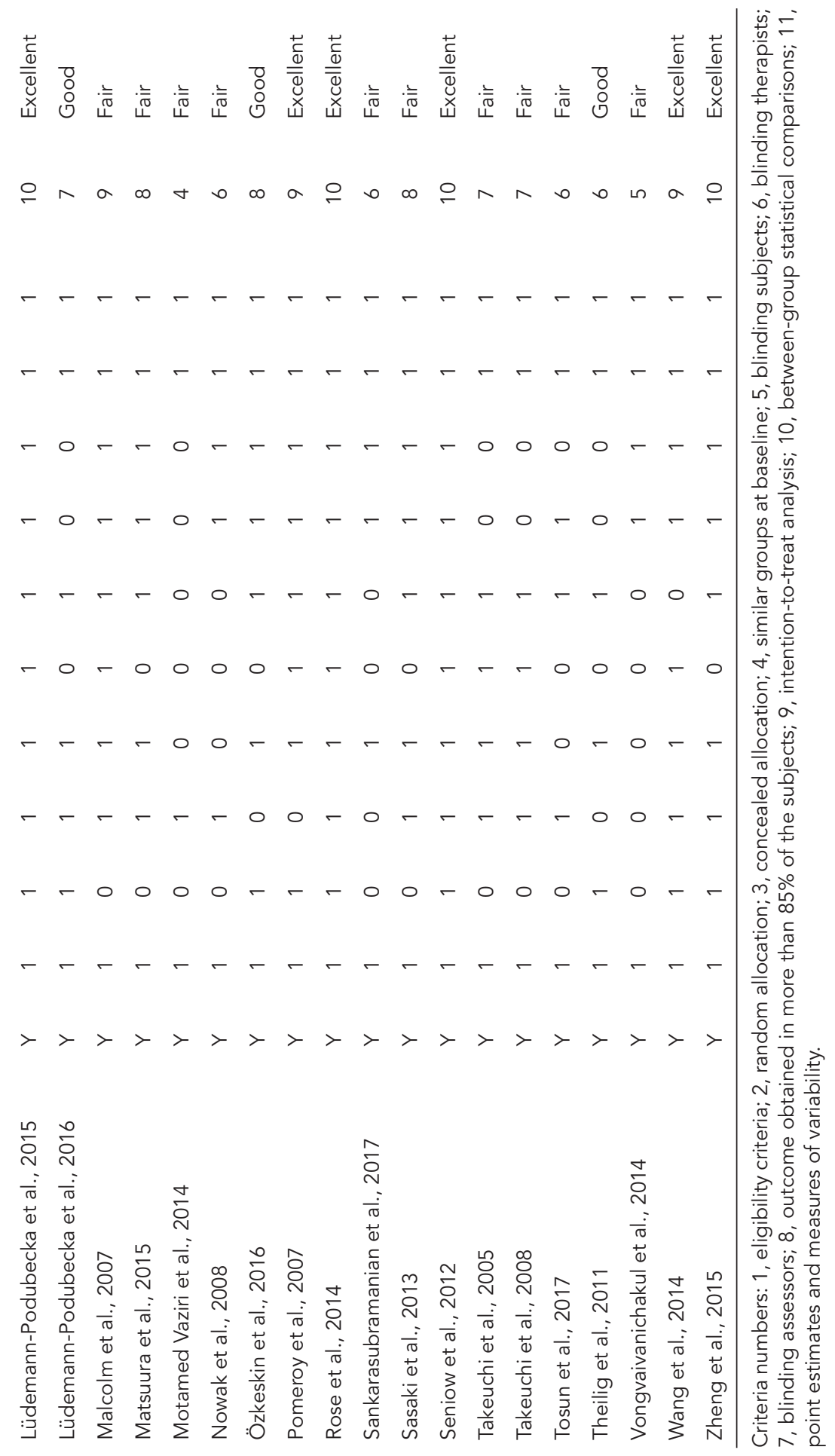




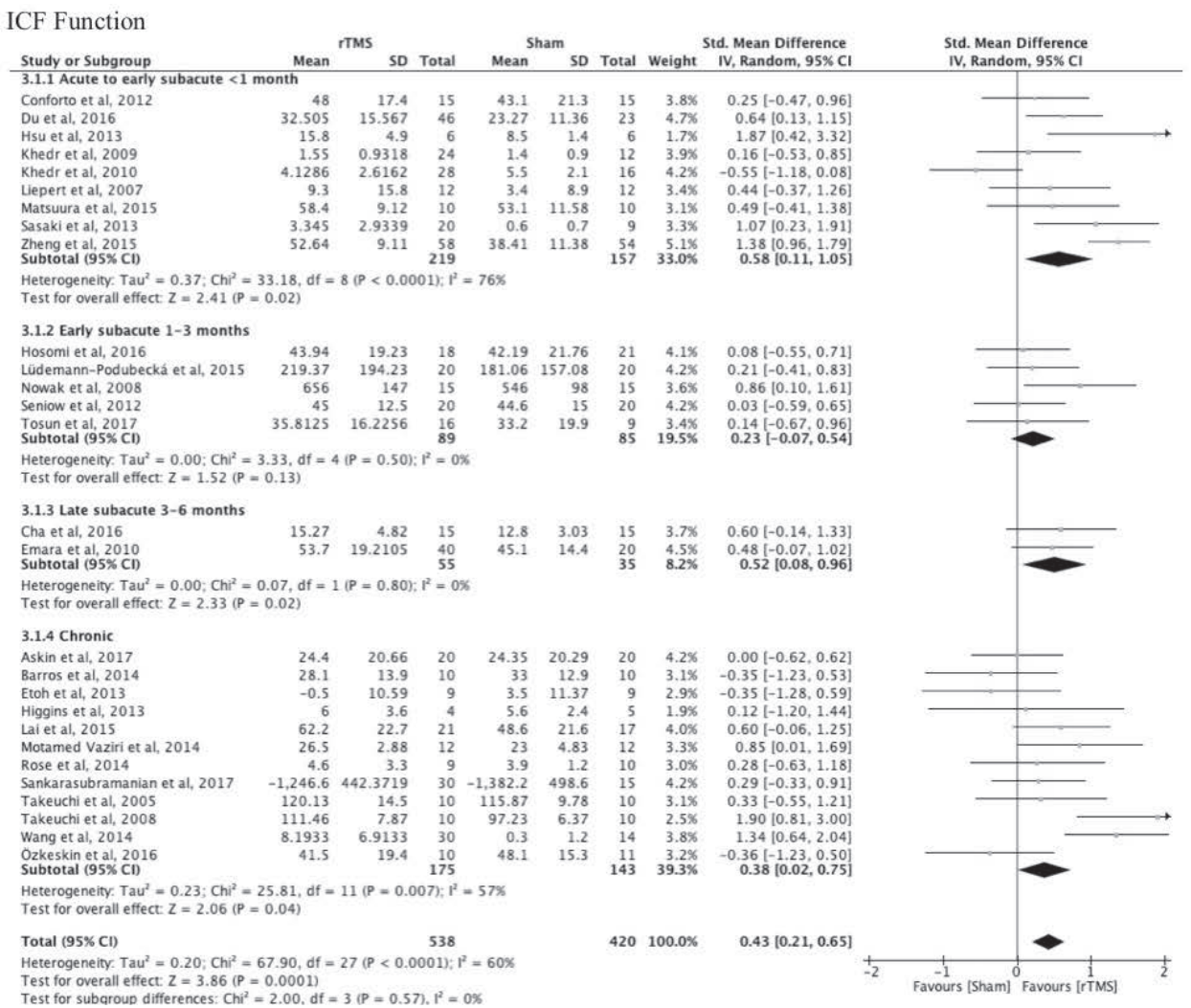

Figure 5.3: Effects of rTMS on the ICF Function domain, comparing different treatment onset times. Estimates of effect size are shown with $95 \%$ Cls.

The standardized mean difference (SMD) and 95\% confidence intervals (Cls); ICF Function measures: Fugl-Meyer Arm, Grip Strength, Finger Tapping and Pinch Force.

and 11-20 sessions), improved upper limb function was found for all the different number of treatment sessions on the ICF function measures (all $p<.05$ ) (Supplementary Figures S5.6.1-S5.6.5 in Appendix 5.6). Subgroup analysis of rTMS alone and rTMS combined with additional therapy showed significant effects on upper limb ICF function measures for both rTMS treatment approaches (Supplementary Figures S5.6.6 and S5.6.7 in Appendix 5.6). In another subgroup analysis, significant mean effect sizes were found for both low- and high frequency rTMS (to the unaffected and affected hemispheres, respectively) (Supplementary Figures S5.6.10 and S5.6.11 in Appendix 5.6). In all the subgroup analyses no significant differences were found between different rTMS post-stroke onset times. 


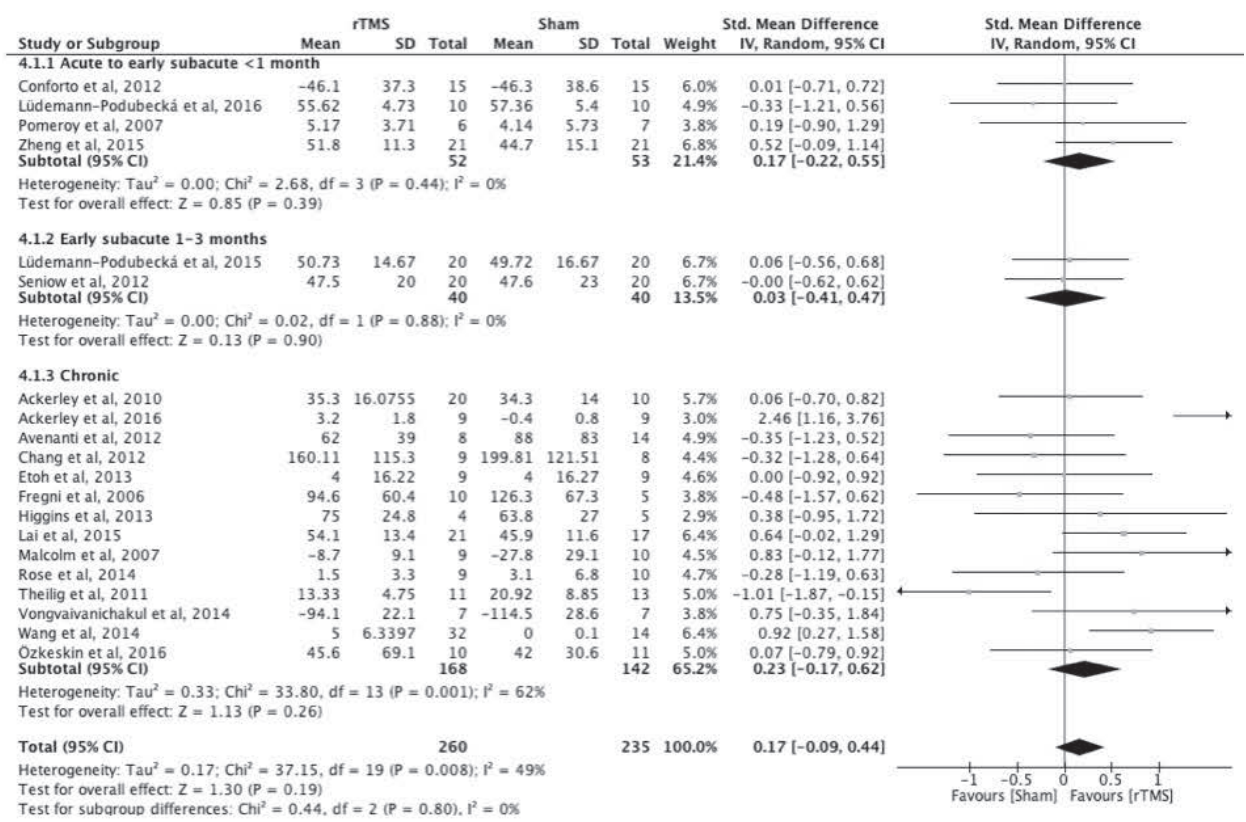

Figure 5.4: Effects of rTMS on the ICF Activity domain, comparing different treatment onset times. Estimates of effect size are shown with $95 \%$ Cls.

The standardized mean difference (SMD) and 95\% confidence intervals (Cls); No studies within 3--6 months post-stroke subgroup; ICF Activity measures: Jebsen Taylor Test, Action Research Arm Test and Wolf Motor Function Test.

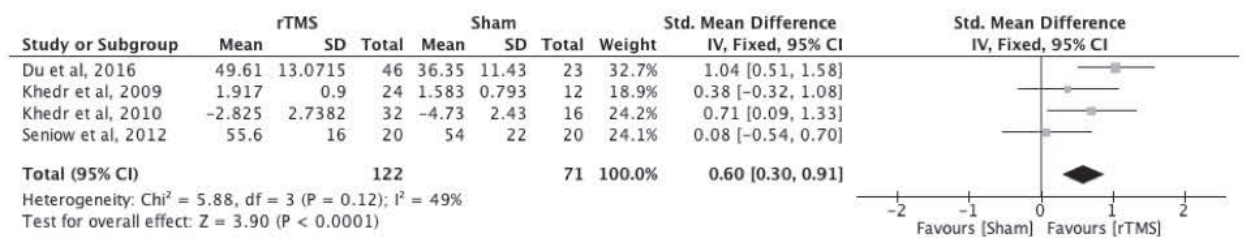

Figure 5.5: Effects of rTMS applied at 1 month with outcome assessment at 3 months post-stroke. Estimates of effect size are shown with $95 \% \mathrm{Cls}$.

The standardized mean difference (SMD) and 95\% confidence intervals (Cls); ICF Function measures: Fugl-Meyer Arm and Grip Strength.

\section{Discussion}

This systematic review and meta-analysis demonstrate that rTMS within one month after stroke leads to greater improvement on the FMA than rTMS applied after one to three months or after six months. In addition, independent from treatment onset time, rTMS seems to have a positive effect on upper limb function if assessed with tests that targeted (body) function specifically, which was not evident with tests assessing activity. Lastly, when rTMS 
treatment was started in the first month after stroke, upper limb function was still improved at three months after stroke, the time of outcome assessment in most acute stroke trials.

\section{Timing of rTMS treatment onset after stroke}

The beneficial effect of rTMS, applied within one month after stroke, on the FMA score have not been previously described. In an earlier systematic review and meta-analysis of rTMS after stroke, which also evaluated the arm/hand motor scales separately for upper limb function, no effect of rTMS followed by upper limb training on motor outcome measures, including the FMA, was found. ${ }^{11}$ However, this systematic review included only eight studies and patients treated within one month after stroke were excluded. A recent randomized sham-controlled trial $(n=199)$ that was published after the search period of our meta-analysis found no difference between active and sham rTMS treatment groups, combined with motor training, on the FMA (nor on the ARAT or WMFT). This lack of difference may be attributed to the inclusion of patients beyond 3 months after stroke. ${ }^{67}$ The results from our meta-analysis also differ from findings from a recent meta-analysis of tDCS treatments after stroke, in which increased capacity of activities of daily living (ADL), but not increased arm function, measured by the FMA, were reported after tDCS. ${ }^{68} \mathrm{~A}$ reason for the discrepancy between these results and our findings may be the difference in included post-stroke time points (ranging between 3 days up to 8 years post-stroke for the meta-analysis of tDCS treatments). The discrepancy could also be attributed to the different mechanisms underlying cortical excitability changes after rTMS and tDCS. TMS can directly induce action potentials, whereas tDCS does not evoke action potentials but modifies neuronal membrane polarization.,69 This can result in different neuromodulatory responses between rTMS and tDCS stimulated neural networks. Another explanation might be that the improvement in ADL capacity is not a reflection of improvement in arm function but of generalized treatment effects. In addition, patients with a non-functional arm may be independent in ADL. ${ }^{70}$

\section{Body function measures}

The FMA, a measure of body function, has recently been recommended as a primary outcome measure for intervention trials targeting the upper limb throughout different phases after stroke. ${ }^{71,72}$ While rTMS improved FMA scores specifically when applied in the first month, this effect was not observed for other body function measures. This may at least partly be explained by the higher number of studies that assessed upper limb function with the FMA ( $n=15)$ than with the other body function measures (RT, FT, GS and PF) ( $n \leq 8)$. Consequently, the low numbers of patients (sample sizes: 6-60) in studies that used other measures and no power calculations may have led to insufficient power to detect differences. 
Another possible explanation may be that the FMA assesses multiple components of the upper limb, such as the shoulder, elbow, wrist, hand, fingers and coordination, and is based on the different sequential stages of motor recovery. According to the FMA stages of recovery (based on the Brunnstrom Approach), basic synergy patterns appear in one of the first stages, and points can be awarded in each stage. ${ }^{73}$ By contrast, other body function measures assess or focus on fine motor control, and points are only awarded when the patient can move freely from the synergy pattern. Consequently, some patients will not be able to perform the fine motor tasks assessed with these scales and possible improvements in distal musculature cannot be captured. However, if rTMS treatment started in the chronic phase post-stroke and outcome was assessed with measures at ICF function level, other than the FMA, these patients displayed a favorable response to the treatment. It is possible that these patients developed compensatory movements to accomplish the function tests, e.g., by using additional trunk movements. ${ }^{74,75}$

\section{Comparisons to previous studies}

Two earlier meta-analyses also performed a subgroup analysis for rTMS effects at different times after stroke. ${ }^{10,76}$ These analyses also showed more pronounced effects of rTMS applied in the (sub)acute phase ( 2 weeks to 6 months) than in the chronic phase (> 6 months) post-stroke. However, these meta-analyses pooled studies with outcome measures at different levels of ICF (i.e. function and activity), which increases methodological variation. Furthermore, not all findings were corrected for multiple comparisons ${ }^{76}$ and few studies selectively included patients at specific post-stroke stages. ${ }^{10}$

Earlier meta-analyses have considered the potential influence of rTMS frequency/site of stimulation, ${ }^{10,76}$ number of sessions ${ }^{76}$ and upper-limb training ${ }^{11}$ on upper limb function. Two meta-analyses revealed more pronounced effects on upper limb function following low-frequency rTMS to the unaffected hemisphere as compared to high-frequency rTMS to the affected hemisphere. ${ }^{10,76}$ Low-frequency rTMS protocols have been more frequently used than high-frequency protocols to promote upper limb recovery, throughout the different post-stroke phases. In the current meta-analysis, both the low- and high-frequency studies revealed significant effects on upper limb function measured by ICF function measures. Outcome measures have not previously been categorized according to their measurement level (ICF) in meta-analyses. Prior studies had shown that five rTMS sessions have the most beneficial effects on upper limb function compared to a single session or more than ten sessions. ${ }^{76}$ In contrast to these findings, our subgroup analyses showed that there were significant beneficial effects on ICF function measures for varying amount of treatment sessions (i.e. single treatment session, 2-10 or 11-20 sessions), however this finding is based on few studies within the different phases of treatment onset post-stroke. 
Regarding additional therapy next to rTMS treatment, one study did not find support that the combination of rTMS with upper-limb training would be more beneficial on upper limb function than upper-limb training alone. ${ }^{11}$ In our analysis, additional therapy combined with rTMS was found to have a similar effect as rTMS alone. However, the effect of specific types or intensity of additional therapy, paired with rTMS, has not been investigated yet.

\section{Outcome measure selection}

To effectively capture the multidimensional aspects of post-stroke dysfunction and recovery, it has been recommended to measure outcome at different levels of function, activity and participation (ICF model). ${ }^{71}$ Outcome measures at the level of function are more directly linked to stroke-related brain changes as compared to outcome measures at the level of activity, which are also strongly affected by cognitive, environmental and personal factors. ${ }^{18,77}$ This could explain why we found no effect of rTMS treatment on activity outcome measures. High heterogeneity and wide confidence intervals of effect sizes were found for some analyses on activity outcome measures, which could also account for the absence of rTMS effects in activity.

It is important that the selected outcome measures within a trial reflect the underlying rationale or mechanism of the intervention under study. Furthermore, interventions targeted at one or more specific parts of the upper limb (i.e. arm, hand, shoulder) should select an outcome measure that is capable of specifically assessing effects on those parts or subtest scores of an outcome measure should be reported to indicate at what level of the upper limb the most significant effects occur. However, for several tests it is not entirely clear to which ICF domain they belong. For example, some outcome measures at activity level (e.g. ARAT and WMFT) also contain a number of test items at function level and vice versa. Effects of interventions which directly influence neural activity, such as rTMS, are probably best assessed with outcome measures that are able to capture the neural recovery process. For motor function, this may be achieved with the FMA. In addition, inclusion of arm/hand motor scales at the level of activity and participation as secondary outcomes can be valuable to evaluate if treatment effects generalize to daily life. Objective kinematic measurements may offer a valuable addition to the existing and widely used outcome measures. These quantitative assessments can provide more detailed insights into key components of motor recovery, such as individual finger movements, smoothness of reaching, force control and trunk displacement. ${ }^{78,79}$ A combination of outcome measures at different ICF domains, including the use of kinematic measures, can also prevent a patient from becoming discouraged if the performance on a particular test fails. 


\section{Study strengths and limitations}

The beneficial effect of rTMS applied in the acute to early subacute phase post-stroke is in agreement with theories on a critical time window post-stroke for obtaining recoveryenhancing effects. ${ }^{14}$ Our review showed that when rTMS was applied in the first month after stroke, a beneficial effect on upper limb function could still be measured at three months post-stroke. A three-month post-stroke assessment has been recommended by the Stroke Recovery and Rehabilitation Roundtable for stroke recovery trials, especially when interventions target neural repair processes, which may be most prevalent during this timeframe. ${ }^{71}$ In addition, assessment after six months can inform on outcome at a stage when spontaneous recovery often reaches a plateau, particularly in more severe strokes. ${ }^{80}$

There are limitations in our review and meta-analysis that need to be reported. Firstly, since our study was dependent on the type and quality of the data in the individual studies, risks of bias that could lead to inflation of the effect size estimates should be acknowledged. Therefore, the results need to be interpreted with caution. There were some examples of risk of bias. In some subgroup analyses only one study was representative of a subgroup. Heterogeneities in the results of the individual studies included in the main analyses were large, as suggested by funnel plot asymmetry. Measurement of effect sizes of treatment was often based on a mixture of change scores and final scores. However, unpublished studies with negative findings may have been missed due to publication bias, may also have led to funnel plot asymmetry. The methodological quality of the studies was fair to excellent, but almost half of the studies were single-blind and did not conceal the treatment allocation or describe the allocation procedure. Nevertheless, our sensitivity analyses showed no significant changes in results when those studies were excluded. Also, we might have missed relevant studies published in non-English languages. Another potential source of bias in clinical research is the type of funding or sponsorship. Although none of the studies were funded by an industrial partner, bias can also result from non-commercial funding sources with specific interests. Secondly, because of the large variations in the study populations, we could not examine possibly confounding effects of differences in demographic and stroke-related characteristics between the studies. Age, gender, level of cognition, depression, severity of impairment and physical activity are examples of confounders that could influence motor performance. Thirdly, due to the limited data we could not adequately account for differences in rTMS protocols and frequencies/sites of stimulation, experimental designs, additional therapy, motor scores (e.g. FMA subscores, clinical versus kinematic measures), and patient inclusion criteria. We focused on effects of rTMS applied at different times post-stroke, whereby investigating the role of (intensity of) additional therapy such as virtual reality therapy and functional task practice, and single rTMS sessions could not be performed. 


\section{Conclusions}

rTMS treatment within the first month after stroke seems more beneficial in increasing upper limb function than after 1-3 months or in the chronic phase post-stroke (> 6 months). Improvements after rTMS can most likely be detected with outcome measures assessing body functions, like the FMA score, than tests at the level of activity (e.g. JTT, ARAT). However, rTMS treatment studies in stroke patients are highly heterogeneous, with varying outcome measures and relatively small sample sizes. Another source of uncertainty is that we are unable to identify whether improved outcomes were primarily caused by rTMS per se or by rTMS in combination with an additional therapy (of a certain intensity). Further research and international cooperation should be undertaken to develop a standardized, core set of measurements for testing upper limb function. We recommend to conduct measurements at the different levels of function, activity (and participation). Future studies should incorporate these standardized tests, include a follow-up measurement at three months after stroke onset (if the trial starts within one month post-stroke), and report their findings in a uniform manner (e.g. using final scores or change scores, and subtest scores).

\section{Funding}

This work was supported by the Netherlands Organization for Scientific Research (VICI 016.130.662).

\section{Acknowledgments}

The authors thank Rosa Callenfels (RC) for her check on the search and assessing the methodological quality of the studies in this paper, and Wim Otte for his advice on the statistical analyses.

\section{References}

1. Kwakkel, G., Kollen, B. \& Wagenaar, R. Long term effects of intensity of upper and lower limb training after stroke: A randomised trial. J. Neurol. Neurosurg. Psychiatry 72, 473-479 (2002).

2. Lai, S. M., Studenski, S., Duncan, P. W. \& Perera, S. Persisting consequences of stroke measured by the stroke impact scale. Stroke 33, 1840-1844 (2002).

3. Sunderland, A. et al. Enhanced physical therapy for arm function after stroke: a one year follow up study. J. Neurol. Neurosurg. Psychiatry 57, 856-858 (1994).

4. Kwakkel, G., Kollen, B. J., Van der Grond, J. V. \& Prevo, A. J. H. Probability of regaining dexterity in the flaccid upper limb: Impact of severity of paresis and time since onset in acute stroke. Stroke 34, 2181-2186 (2003). 
5. Bernhardt, J. et al. Moving rehabilitation research forward: Developing consensus statements for rehabilitation and recovery research. Int. J. Stroke 11, 454-458 (2016).

6. Sebastianelli, L. et al. Low-frequency rTMS of the unaffected hemisphere in stroke patients: A systematic review. Acta Neurol. Scand. 136, 585-605 (2017).

7. Pascual-Leone, A. et al. Study and modulation of human cortical excitability with transcranial magnetic stimulation. J. Clin. Neurophysiol. 15, 333-343 (1998).

8. Di Pino, G. et al. Modulation of brain plasticity in stroke: a novel model for neurorehabilitation. Nat. Rev. Neurol. 10, 597-608 (2014).

9. Le, Q., Qu, Y., Tao, Y. \& Zhu, S. Effects of repetitive transcranial magnetic stimulation on hand function recovery and excitability of the motor cortex after stroke: A meta-analysis. Am. J. Phys. Med. Rehabil. 93, 422-430 (2014).

10. Hsu, W. Y., Cheng, C. H., Liao, K. K., Lee, I. H. \& Lin, Y. Y. Effects of repetitive transcranial magnetic stimulation on motor functions in patients with stroke: A meta-analysis. Stroke 43, 1849-1857 (2012).

11. Graef, P., Dadalt, M. L. R., da Silva Rodrigués, D. A. M., Stein, C. \& de Souza Pagnussat, A. Transcranial magnetic stimulation combined with upper-limb training for improving function after stroke: A systematic review and meta-analysis. J. Neurol. Sci. 369, 149-158 (2016).

12. Hao, Z., Wang, D., Zeng, Y. \& Liu, M. Repetitive transcranial magnetic stimulation for improving function after stroke. Cochrane Libr. 5, CD008862 (2013).

13. Bernhardt, J., English, C., Johnson, L. \& Cumming, T. B. Early mobilization after stroke: early adoption but limited evidence. Stroke 46, 1141-1146 (2015).

14. Krakauer, J. W., Carmichael, S. T., Corbett, D. \& Wittenberg, G. F. Getting neurorehabilitation right: what can be learned from animal models? Neurorehabil. Neural Repair 26, 923-931 (2012).

15. Biernaskie, J. Efficacy of rehabilitative experience declines with time after focal ischemic brain injury. J. Neurosci. 24, 1245-1254 (2004).

16. Kwakkel, G., Kollen, B. \& Lindeman, E. Understanding the pattern of functional recovery after stroke: facts and theories. Restor. Neurol. Neurosci. 22, 281-299 (2004).

17. World Health Organization (WHO). International classification of functioning, disability and health. World Health Organization 1-15 (2003). Available at: http://www.who.int/classifications/icf/icfchecklist. pdf?ua=1.

18. Schepers, V. P. M., Ketelaar, M., van de Port, I. G. L., Visser-Meily, J. M. A. \& Lindeman, E. Comparing contents of functional outcome measures in stroke rehabilitation using the International Classification of Functioning, Disability and Health. Disabil. Rehabil. 29, 221-230 (2007).

19. Moher D, Liberati A, T. J. and A. D. The PRISMA group. Preferred reporting items for systematic reviews and meta-analyses: the PRISMA statement. Ann. Intern. Med. 151, 264-269 (2009).

20. Foley, N. C., Bhogal, S. K., Teasell, R. W., Bureau, Y. \& Speechley, M. R. Estimates of quality and reliability with the physiotherapy evidence-based database scale to assess the methodology of randomized controlled trials of pharmacological and nonpharmacological interventions. Phys. Ther. 86, 817-824 (2006).

21. Foley, N. C., Teasell, R. W., Bhogal, S. K. \& Speechley, M. R. Stroke Rehabilitation Evidence-Based Review: methodology. Top. Stroke Rehabil. 10, 1-7 (2003).

22. Bernhardt, J. et al. Agreed definitions and a shared vision for new standards in stroke recovery research : The Stroke Recovery and Rehabilitation Roundtable taskforce. Int. J. Stroke 12, 444-450 (2017).

23. Dancause, N. \& Nudo, R. J. Shaping plasticity to enhance recovery after injury. Prog Brain Res. 192, 273-295 (2011).

24. Higgins, J. P. \& Green, S. Cochrane Handbook for Systematic Reviews of Interventions: Cochrane Book Series (2008). doi:10.1002/9780470712184 
25. Cohen, J. Statistical power analysis for the behavioral sciences. Statistical Power Analysis for the Behavioral Sciences 2nd, 567 (1988).

26. The Nordic Cochrane Centre. Review Manager. Cochrane Collaboration 1-43 (2014).

27. Landis, J. R. \& Koch, G. G. The measurement of observer agreement for categorical data published by: International Biometric Society Stable URL : http://www.jstor.org/stable/2529310. Society 33, 159-174 (2008).

28. Nowak, D. A. et al. Effects of low-frequency repetitive transcranial magnetic stimulation of the contralesional primary motor cortex on movement kinematics and neural activity in subcortical stroke. Arch. Neurol. 65, 741-747 (2008).

29. Sankarasubramanian, V. et al. Clinical Neurophysiology Inhibition versus facilitation of contralesional motor cortices in stroke : Deriving a model to tailor brain stimulation. Clin. Neurophysiol. 128, 892-902 (2017).

30. Etoh, S. et al. Effects of repetitive transcranial magnetic stimulation on repetitive facilitation exercises of the hemiplegic hand in chronic stroke patients. J. Rehabil. Med. 45, 843-847 (2013).

31. Liepert, J., Zittel, S. \& Weiller, C. Improvement of dexterity by single session low-frequency repetitive transcranial magnetic stimulation over the contralesional motor cortex in acute stroke: a doubleblind placebo-controlled crossover trial. Restor. Neurol. Neurosci. 25, 461-465 (2007).

32. Ludemann-Podubecka, J., Bosl, K. \& Nowak, D. A. Inhibition of the contralesional dorsal premotor cortex improves motor function of the affected hand following stroke. Eur. J. Neurol. 23, 823-830 (2016).

33. Ackerley, S. J., Stinear, C. M., Barber, P. A. \& Byblow, W. D. Combining theta burst stimulation with training after subcortical stroke. Stroke 41, 1568-1572 (2010).

34. Matsuura, A., Onoda, K., Oguro, H. \& Yamaguchi, S. Magnetic stimulation and movement-related cortical activity for acute stroke with hemiparesis. Eur. J. Neurol. 22, 1526-1532 (2015). doi:10.1111/ ene. 12776

35. Barros, S. V, Borba Costa dos Santos, R., Borba dos Santos, P., Cabral, M. E. \& Monte-Silva, K. Efficacy of coupling repetitive transcranial magnetic stimulation and physical therapy to reduce upper-limb spasticity in patients with stroke: A randomized controlled trial. Arch. Phys. Med. Rehabil. 95, 222-229 (2014).

36. Chang, W. H. et al. RTMS with motor training modulates cortico-basal ganglia-thalamocortical circuits in stroke patients. Restor. Neurol. Neurosci. 30, 179-189 (2012).

37. Theilig, S., Podubecka, J., Bösl, K., Wiederer, R. \& Nowak, D. A. Functional neuromuscular stimulation to improve severe hand dysfunction after stroke: Does inhibitory rTMS enhance therapeutic efficiency? Exp. Neurol. 230, 149-155 (2011).

38. Takeuchi, N. et al. Inhibition of the unaffected motor cortex by $1 \mathrm{HZ}$ repetitive transcranial magnetic stimulation enhances motor performance and training effect of the paretic hand in patients with chronic stroke. J. Rehabil. Med. 40, 298-303 (2008).

39. Fregni, F. et al. A sham-controlled trial of a 5-day course of repetitive transcranial magnetic stimulation of the unaffected hemisphere in stroke patients. Stroke 37, 2115-2122 (2006).

40. Takeuchi, N., Chuma, T., Matsuo, Y., Watanabe, I. \& Ikoma, K. Repetitive transcranial magnetic stimulation of contralesional primary motor cortex improves hand function after stroke. Stroke 36, 2681-2686 (2005).

41. Motamed Vaziri, P. et al. Low frequency repetitive transcranial magnetic stimulation to improve motor function and grip force of upper limbs of patients with hemiplegia. Iran. Red Crescent Med. J. 16, e13579 (2014).

42. Malcolm, M. P. et al. Repetitive transcranial magnetic stimulation as an adjunct to constraint-induced therapy: An exploratory randomized controlled trial. Am. J. Phys. Med. Rehabil. 86, 707-715 (2007). 
43. Avenanti, a, Coccia, M., Ladavas, E., Provinciali, L. \& Ceravolo, M. G. Low-frequency rTMS promtes use-dependent motor plasticity in chronic stroke. A randomized trial. Neurology 78, 256-264 (2012).

44. Wang, C. C. et al. Inhibitory repetitive transcranial magnetic stimulation of the contralesional premotor and primary motor cortices facilitate poststroke motor recovery. Restor. Neurol. Neurosci. 32, 825-835 (2014).

45. Özkeskin, M., Öztürk, V., Çakmur, R. \& Kara, B. Navigated repetitive transcranial magnetic stimulation or brunnstrom hand manipulation: Which treatment is more effective in stroke cases? J. Neurol. Sci. 33, 361-372 (2016).

46. Lai, C. J. et al. Corticospinal integrity and motor impairment predict outcomes after excitatory repetitive transcranial magnetic stimulation: A preliminary study. Arch. Phys. Med. Rehabil. 96, 69-75 (2015).

47. Aşkın, A., Tosun, A. \& Demirdal, Ü. S. Effects of low-frequency repetitive transcranial magnetic stimulation on upper extremity motor recovery and functional outcomes in chronic stroke patients: A randomized controlled trial. Somatosens. Mot. Res. 34, 102-107 (2017).

48. Ackerley, S. J. et al. Primed physical therapy enhances recovery of upper limb function in chronic stroke patients. Neurorehabil. Neural Repair 30, 339-48 (2016).

49. Rose, D. K., Patten, C., Mcguirk, T. E., Lu, X. \& Triggs, W. J. Does Inhibitory Repetitive Transcranial Magnetic Stimulation Augment Functional Task Practice to Improve Arm Recovery in Chronic Stroke? Stroke Res. Treat. 2014, 305236 (2014).

50. Vongvaivanichakul, P., Tretriluxana, J., Bovonsunthonchai, S., Pakaprot, N. \& Laksanakorn, W. Reach-to-grasp training in individuals with chronic stroke augmented by low-frequency repetitive transcranial magnetic stimulation. J. Med. Assoc. Thai. 97, 3-8 (2014).

51. Higgins, J., Koski, L. \& Xie, H. Combining rTMS and task-oriented training in the rehabilitation of the arm after stroke: A pilot randomized controlled trial. Stroke Res. Treat. 2013, 539146 (2013).

52. Hosomi, K. et al. Daily repetitive transcranial magnetic stimulation for poststroke upper limb paresis in the subacute period. J. Stroke Cerebrovasc. Dis. 25, 1655-1664 (2016).

53. Lüdemann-podubecká, J., Bösl, K., Theilig, S., Wiederer, R. \& Alexander, D. The effectiveness of $1 \mathrm{~Hz}$ rTMS over the primary motor area of the unaffected hemisphere to improve hand function after stroke depends on hemispheric dominance. Brain Stimul. 8, 823-830 (2015).

54. Seniów, J. et al. Transcranial magnetic stimulation combined with physiotherapy in rehabilitation of poststroke hemiparesis. Neurorehabil. Neural Repair 26, 1072-1079 (2012).

55. Emara, T. H. et al. Repetitive transcranial magnetic stimulation at $1 \mathrm{~Hz}$ and $5 \mathrm{~Hz}$ produces sustained improvement in motor function and disability after ischaemic stroke. Eur. J. Neurol. 17, 1203-1209 (2010).

56. Cha, H. G. \& Kim, M. K. Effects of repetitive transcranial magnetic stimulation on arm function and decreasing unilateral spatial neglect in subacute stroke: a randomized controlled trial. Clin. Rehabil. 30, 649-656 (2016).

57. Du, J. et al. Effects of repetitive transcranial magnetic stimulation on motor recovery and motor cortex excitability in patients with stroke: a randomized controlled trial. Eur. J. Neurol. 23, 1666-1672 (2016).

58. Zheng, C. J., Liao, W. J. \& Xia, W. G. Effect of combined low-frequency repetitive transcranial magnetic stimulation and virtual reality training on upper limb function in subacute stroke: a double-blind randomized controlled trail. J. Huazhong Univ. Sci. Technol. Med. Sci. 35, 248-254 (2015).

59. Sasaki, N., Mizutani, S., Kakuda, W. \& Abo, M. Comparison of the effects of high- and low-frequency repetitive transcranial magnetic stimulation on upper limb hemiparesis in the early phase of stroke. J. Stroke Cerebrovasc. Dis. 22, 413-418 (2013). 
60. Conforto, A. B. et al. Transcranial magnetic stimulation in mild to severe hemiparesis early after stroke: A proof of principle and novel approach to improve motor function. J. Neurol. 259, 1399-1405 (2012).

61. Pomeroy, V. M. et al. Transcranial magnetic stimulation and muscle contraction to enhance stroke recovery: A randomized proof-of-principle and feasibility investigation. Neurorehabil. Neural Repair 21, 509-517 (2007).

62. Khedr, E. M., Abdel-Fadeil, M. R., Farghali, A. \& Qaid, M. Role of 1 and $3 \mathrm{~Hz}$ repetitive transcranial magnetic stimulation on motor function recovery after acute ischaemic stroke. Eur. J. Neurol. 16, 1323-1330 (2009).

63. Khedr, E. M., Etraby, A. E., Hemeda, M., Nasef, A. M. \& Razek, A. A. E. Long-term effect of repetitive transcranial magnetic stimulation on motor function recovery after acute ischemic stroke. Acta Neurol. Scand. 121, 30-37 (2010).

64. Hsu, Y. F. et al. Intermittent theta burst stimulation over ipsilesional primary motor cortex of subacute ischemic stroke patients: A pilot study. Brain Stimul. 6, 166-174 (2013).

65. Blesneag, A. $\vee$ et al. Low-frequency $r T M S$ in patients with subacute ischemic stroke : clinical evaluation of short and long-term outcomes and neurophysiological assessment of cortical excitability. J. Med. Life 8, 378-387 (2015).

66. Tosun, A. et al. Effects of Low-Frequency repetitive transcranial magnetic stimulation and neuromuscular electrical stimulation on upper extremity motor recovery in the early period after stroke: A preliminary study. Top. Stroke Rehabil. 24, 361-367 (2017).

67. Harvey, R. L. et al. Randomized Sham-Controlled Trial of Navigated Repetitive Transcranial Magnetic Stimulation for Motor Recovery in Stroke. Stroke 49, 2138-2146 (2018).

68. Elsner, B., Kwakkel, G., Kugler, J. \& Mehrholz, J. Transcranial direct current stimulation (tDCS) for improving capacity in activities and arm function after stroke: a network meta-analysis of randomised controlled trials. J. Neuroeng. Rehabil. 14, 95 (2017).

69. Kubis, N. Non-Invasive Brain Stimulation to Enhance Post-Stroke Recovery. Front. Neural Circuits 10, 56 (2016).

70. Rand, D. \& Eng, J. J. Disparity between functional recovery and daily use of the upper and lower extremities during subacute stroke rehabilitation. Neurorehabil. Neural Repair 26, 76-84 (2012).

71. Kwakkel, G. Standardised measurement of sensorimotor recovery in stroke trials: consensus-based core recommendations from the Stroke Recovery and Rehabilitation Roundtable (SRRR). Int. J. Stroke 12, 451-461 (2017).

72. Santisteban, L. et al. Upper limb outcome measures used in stroke rehabilitation studies: A systematic literature review. PLoS One 11, e0154792 (2016).

73. Gladstone, D. J., Danells, C. J. \& Black, S. E. The fugl-meyer assessment of motor recovery after stroke: A critical review of its measurement properties. Neurorehabil. Neural Repair 16, 232-240 (2002).

74. Buma, F., Kwakkel, G. \& Ramsey, N. Understanding upper limb recovery after stroke. Restor. Neurol. Neurosci. 31, 707-722 (2013).

75. Subramanian, S. K., Yamanaka, J., Chilingaryan, G. \& Levin, M. F. Validity of movement pattern kinematics as measures of arm motor impairment poststroke. Stroke 41, 2303-2308 (2010).

76. Zhang, L. et al. Short- and long-term effects of repetitive transcranial magnetic stimulation on upper limb motor function after stroke: a systematic review and meta-analysis. Clin. Rehabil. 31, 1137-1153 (2017).

77. Langhorne, P., Bernhardt, J. \& Kwakkel, G. Stroke rehabilitation. Lancet 377, 1693-1702 (2011).

78. Alt Murphy, M., Willén, C. \& Sunnerhagen, K. S. Responsiveness of upper extremity kinematic measures and clinical improvement during the first three months after stroke. Neurorehabil. Neural Repair 27, 844-853 (2013). 
79. Térémetz, M., Colle, F., Hamdoun, S., Maier, M. A. \& Lindberg, P. G. A novel method for the quantification of key components of manual dexterity after stroke. J. Neuroeng. Rehabil. 12, 64 (2015).

80. Duncan, P. W., Jorgensen, H. S. \& Wade, D. T. Outcome measures in acute stroke trials: A systematic review and some recommendations to improve practice. Stroke 31, 1429-1438 (2000). 


\section{Appendix 5.1 Search strategy}

\section{Embase}

'cerebrovascular accident'/exp

'brain ischemia'/exp

'brain hemorrhage'/exp

'cerebrovascular accident' OR 'cerebral ischemia' OR 'cerebral ischaemia' OR 'brain ischemia' OR 'brain ischaemia' OR 'brain infarction' OR 'intracranial hemorrhage' OR 'intracranial haemorrhage' OR 'intracerebral hemorrhage' OR 'intracerebral haemorrhage' OR 'intracranial embolism' OR 'intracranial thrombus' OR 'lacunar infarct' OR 'lacunar stroke' OR 'post stroke' OR 'poststroke' OR 'brain vascular accident' OR 'cerebral infarct' OR 'brain hemorrhage' OR 'brain haemorrhage'

AND

'transcranial magnetic stimulation'/exp

'Transcranial Magnetic' OR 'repetitive transcranial' OR 'rTMS' OR 'TBS' OR 'theta burst'

AND

'upper limb'/exp OR

'upper limb function' OR 'upper extremity' OR 'upper extremities' OR 'arm' OR 'arms' OR 'hand' OR 'hands' or 'paresis' OR 'pareses'

\section{Pubmed}

(("Transcranial Magnetic Stimulation"[Mesh] OR

Transcranial Magnetic[Title/Abstract] OR

repetitive transcranial[Title/Abstract] OR

rTMS[Title/Abstract] OR

TBS[Title/Abstract] OR

theta burst[Title/Abstract] OR

paired pulse magnet*[Title/Abstract] OR

paired pulse tms[Title/Abstract] OR

paired associative[Title/Abstract])

AND

("Stroke"[Mesh] OR "Brain Ischemia"[Mesh] OR

"Intracranial Hemorrhages"[Mesh] OR

stroke[Title/Abstract] OR

CVA[Title/Abstract] OR

CVAs[Title/Abstract] OR 
cerebrovascular accident*[Title/Abstract] OR

cerebral ischemia*[Title/Abstract] OR

cerebral ischaemia*[Title/Abstract] OR

brain ischemia*[Title/Abstract] OR

brain ischaemia*[Title/Abstract] OR

brain infarction*[Title/Abstract] OR

intracranial hemorrhage*[Title/Abstract] OR

intracranial haemorrhage*[Title/Abstract] OR

intracerebral hemorrhage ${ }^{\star}$ [Title/Abstract] OR

intracerebral haemorrhage ${ }^{\star}[$ Title/Abstract] OR

intracranial embolism*[Title/Abstract] OR

intracranial thromb*[Title/Abstract] OR

lacunar infarct*[Title/Abstract] OR

lacunar stroke*[Title/Abstract] OR

poststroke[Title/Abstract] OR

post stroke[Title/Abstract] OR

brain vascular accident*[Title/Abstract] OR

cerebral infarct*[Title/Abstract] OR

brain hemorrhage*[Title/Abstract] OR

brain haemorrhage*[Title/Abstract]))

AND

("Upper Extremity"[Mesh] OR

upper extremit*[Title/Abstract] OR

arm[Title/Abstract] OR arms[Title/Abstract] OR

hand[Title/Abstract] OR hands[Title/Abstract] OR

"Paresis"[Mesh] OR paresis[Title/Abstract] OR

pareses[Title/Abstract])

\section{Cochrane Library}

cerebrovascular accident or cerebral ischemia or cerebral ischaemia or brain ischemia or brain ischaemia or brain infarction or intracranial hemorrhage or intracranial haemorrhage or intracerebral hemorrhage or intracerebral haemorrhage or intracranial embolism or intracranial thrombus or lacunar infarct or lacunar stroke or post stroke or poststroke or brain vascular accident or cerebral infarct or brain hemorrhage or brain haemorrhage

Transcranial Magnetic or repetitive transcranial or rTMS or TBS or theta burst

Upper Extremity or upper extremit* or arm or arms or hand or hands or Paresis or paresis or pareses or upper limb* 


\section{Appendix 5.2 Effects of rTMS on the FMA scale}

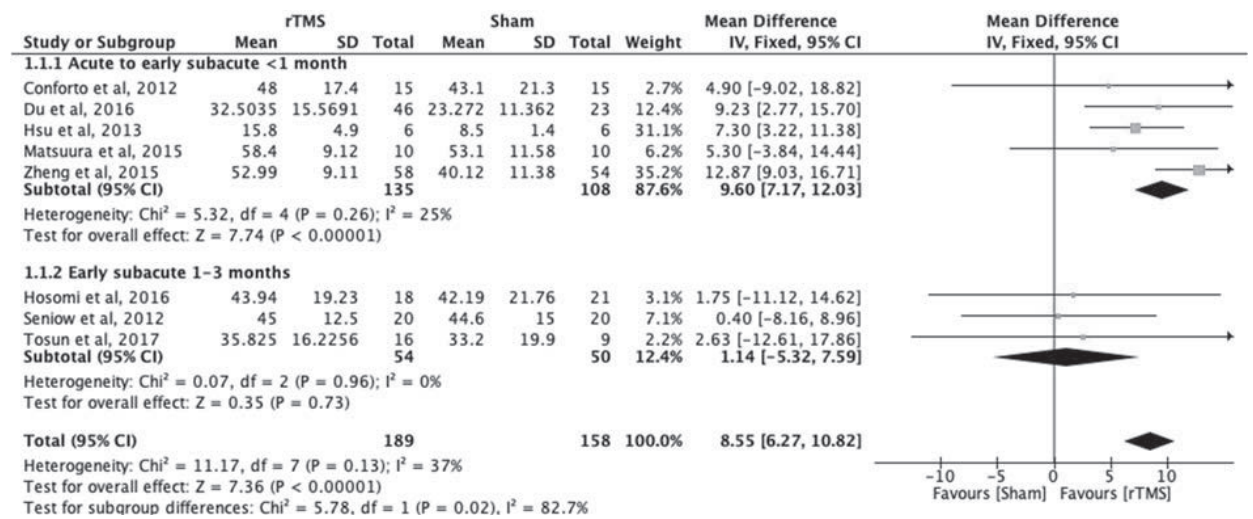

Figure S5.2.1: Effects of rTMS on the FMA scale, comparing the acute to early subacute ( $<1$ month) and early subacute (1-3 months) treatment onset times.

Estimates of effect size are shown with $95 \% \mathrm{Cls}$. MD: mean difference; Cls: 95\% confidence intervals.

\begin{tabular}{|c|c|c|c|c|c|c|c|c|c|c|}
\hline \multirow[b]{2}{*}{ Study or Subgroup } & \multicolumn{2}{|r|}{ rTMS } & \multicolumn{4}{|c|}{ Sham } & \multirow[b]{2}{*}{ Weight } & \multirow{2}{*}{$\begin{array}{l}\text { Mean Difference } \\
\text { IV, Random, } 95 \% \mathrm{CI}\end{array}$} & \multirow{2}{*}{\multicolumn{2}{|c|}{$\begin{array}{l}\text { Mean Difference } \\
\text { IV, Random, } 95 \% \mathrm{CI}\end{array}$}} \\
\hline & Mean & SD & Total & Mean & SD & Total & & & & \\
\hline Conforto et al, 2012 & 48 & 17.4 & 15 & 43.1 & 21.3 & 15 & $3.8 \%$ & $4.90[-9.02,18.82]$ & & \\
\hline Du et al, 2016 & 32.5035 & 15.5691 & 46 & 23.272 & 11.362 & 23 & $9.0 \%$ & $9.23[2.77,15.70]$ & & \\
\hline Matsuura et al, 2015 & 58.4 & 9.12 & 10 & 53.1 & 11.58 & 10 & $6.5 \%$ & $5.30[-3.84,14.44]$ & & \\
\hline $\begin{array}{l}\text { Zheng et al, } 2015 \\
\text { Subtotal ( } 95 \% \mathrm{CI})\end{array}$ & 52.99 & 9.11 & $\begin{array}{r}58 \\
135\end{array}$ & 40.12 & 11.38 & $\begin{array}{r}54 \\
108\end{array}$ & $\begin{array}{l}11.8 \% \\
42.7 \%\end{array}$ & $\begin{array}{r}12.87[9.03,16.71] \\
9.31[6.27,12.34]\end{array}$ & & \\
\hline \multicolumn{11}{|c|}{$\begin{array}{l}\text { Heterogeneity: } \mathrm{Tau}^{2}=2.95 ; \mathrm{Ch}^{2}=5.32, \mathrm{df}=4(\mathrm{P}=0.26) ; \mathrm{I}^{2}=25 \% \\
\text { Test for overall effect: } \mathrm{Z}=6.00(\mathrm{P}<0.00001)\end{array}$} \\
\hline \multicolumn{11}{|l|}{ 2.1.3 Chronic } \\
\hline Askin et al, 2017 & 24.4 & 20.66 & 20 & 24.35 & 20.29 & 20 & $4.3 \%$ & $0.05[-12.64,12.74]$ & & \\
\hline Barros et al, 2014 & 28.1 & 13.9 & 10 & 33 & 12.9 & 10 & $4.8 \%$ & $-4.90[-16.65,6.85]$ & & \\
\hline Etoh et al, 2013 & -0.5 & 10.59 & 9 & 3.5 & 11.37 & 9 & $5.8 \%$ & $-4.00[-14.15,6.15]$ & & \\
\hline Motamed Vaziri et al, 2014 & 26.5 & 2.88 & 12 & 23 & 4.83 & 12 & $12.5 \%$ & $3.50[0.32,6.68]$ & & \\
\hline Rose et al, 2014 & 4.6 & 3.3 & 9 & 3.9 & 1.2 & 10 & $13.3 \%$ & $0.70[-1.58,2.98]$ & & \\
\hline Wang et al, 2014 & 8.1933 & 6.9133 & 30 & 0.3 & 1.2 & 14 & $13.1 \%$ & $7.89[5.34,10.45]$ & & $\longrightarrow$ \\
\hline \multicolumn{11}{|c|}{$\begin{array}{l}\text { Heterogeneity: } \text { Tau }^{2}=13.88 ; \mathrm{Ch}^{2}=23.21, \mathrm{df}=6(\mathrm{P}=0.0007) ; \mathrm{I}^{2}=74 \% \\
\text { Test for overall effect: } Z=0.93(P=0.35)\end{array}$} \\
\hline Total $(95 \% \mathrm{Cl})$ & & & 235 & & & 194 & $100.0 \%$ & $4.61[1.44,7.77]$ & & \\
\hline \multicolumn{9}{|c|}{$\begin{array}{l}\text { Heterogeneity: } \operatorname{Tau}^{2}=18.17 ; \mathrm{Ch}^{2}=47.11, \mathrm{df}=11(\mathrm{P}<0.00001) ; \mathrm{I}^{2}=77 \% \\
\text { Test for overall effect: } Z=2.85(P=0.004) \\
\text { Test for subqroup differences: } C h \mathrm{I}^{2}=9.17, \mathrm{df}=1(\mathrm{P}=0.002), \mathrm{I}^{2}=89.1 \%\end{array}$} & $-10 \stackrel{-1}{-5}{ }^{1}{ }^{0}$ & $\begin{array}{c}5 \\
5 \\
\text { Favours [rTMS] }\end{array}$ \\
\hline
\end{tabular}

Figure S5.2.2: Effects of rTMS on the FMA scale, comparing the acute to early subacute ( $<1$ month) and chronic (> 6 months) treatment onset times.

Estimates of effect size are shown with 95\% Cls. MD: mean difference; Cls: 95\% confidence intervals. 


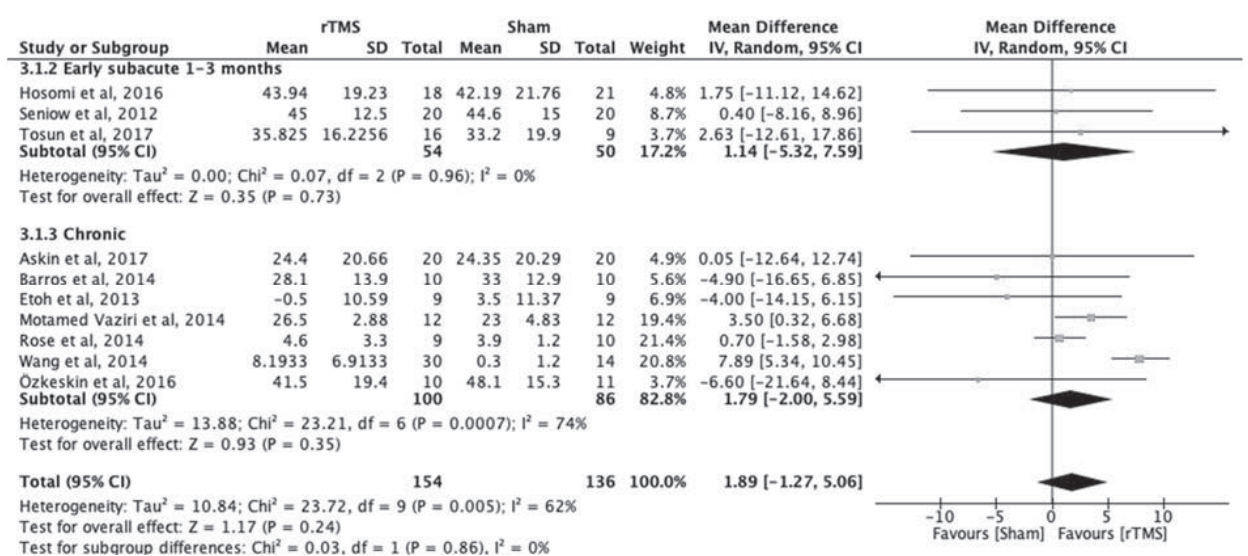

Figure S5.2.3: Effects of rTMS on the FMA scale, comparing the early subacute (1-3 months) and chronic (> 6 months) treatment onset times.

Estimates of effect size are shown with 95\% Cls. MD: mean difference; Cls: 95\% confidence intervals. 


\section{Appendix 5.3 Effects of rTMS on different scales comparing different treatment onset times}

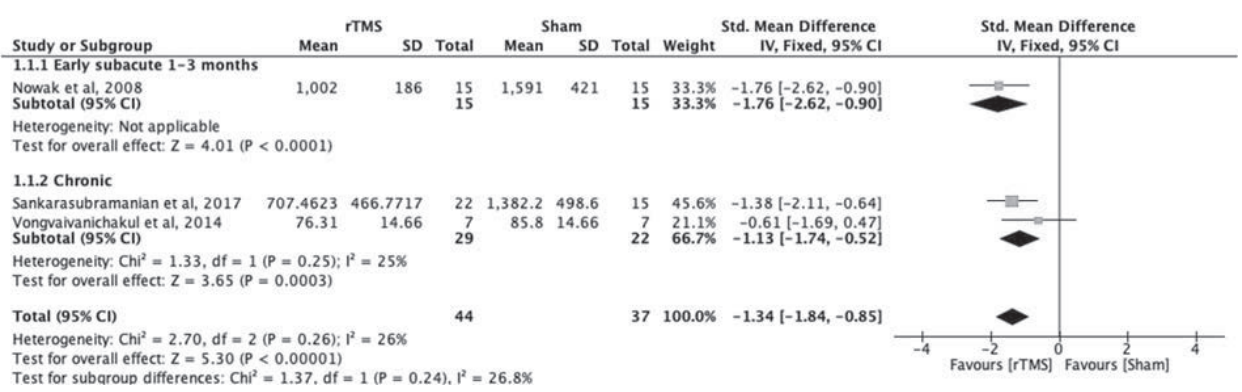

Figure S5.3.1: Effects of rTMS on the Reaching Time (RT) scale, comparing different treatment onset times.

Estimates of effect size are shown with $95 \% \mathrm{Cls}$. SMD: standardized mean difference; Cls: $95 \%$ confidence intervals.

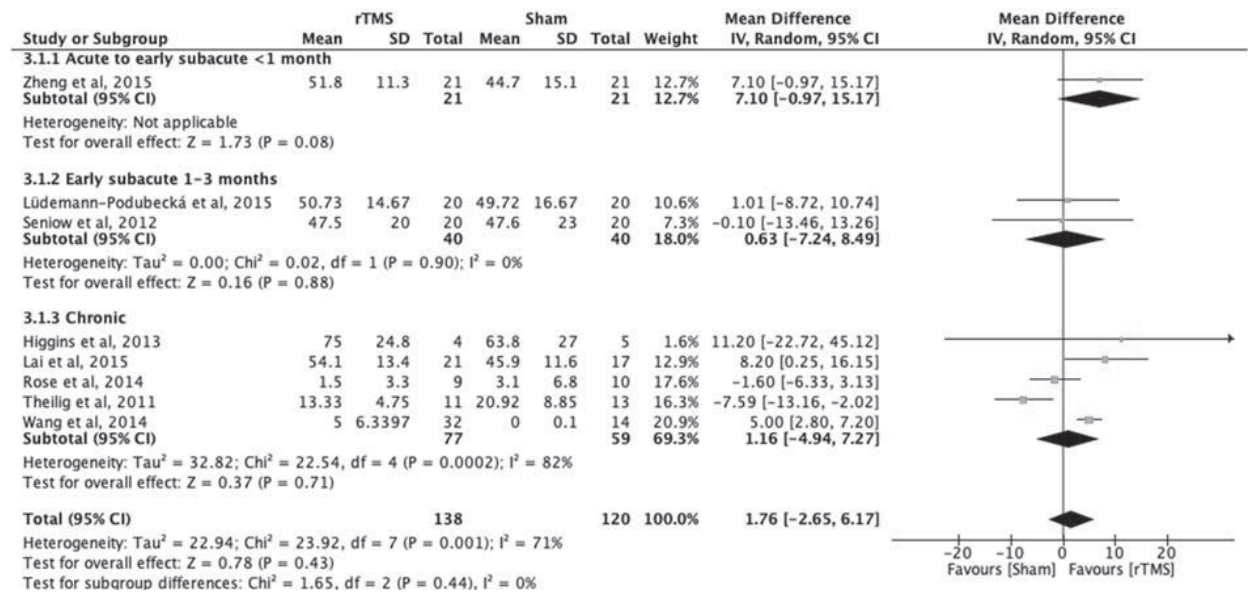

Figure S5.3.2: Effects of rTMS on the Finger Tapping (FT) scale, comparing different treatment onset times.

Estimates of effect size are shown with 95\% Cls. SMD: standardized mean difference; Cls: 95\% confidence intervals. 


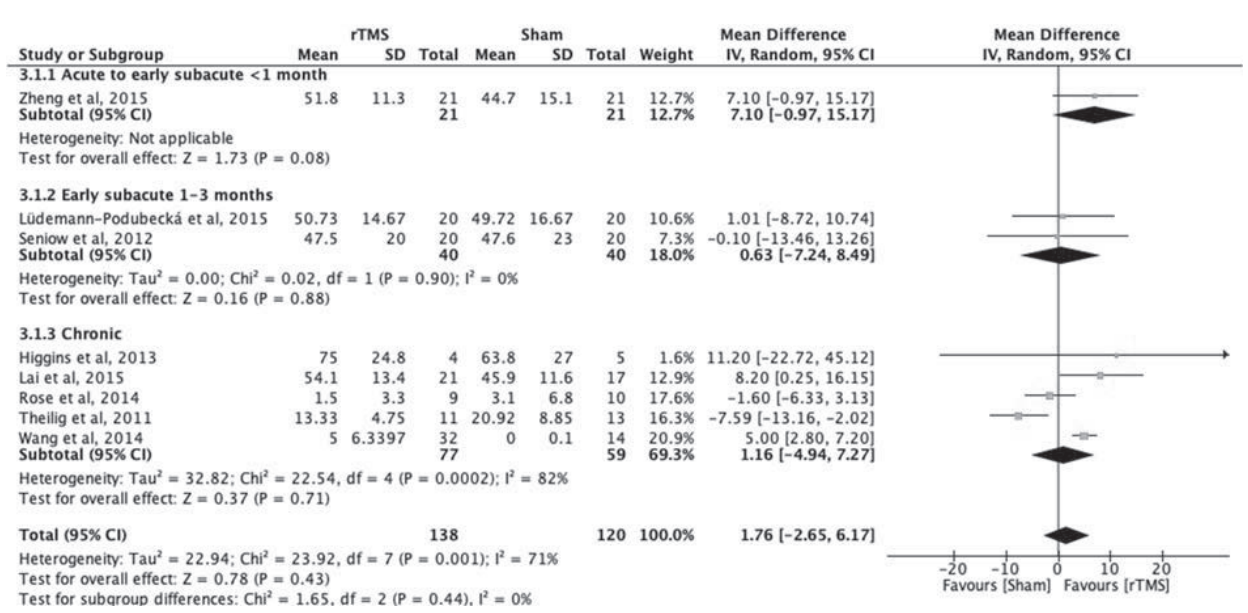

Figure S5.3.3: Effects of rTMS on the Wolf Motor Function Test (WMFT) scale, comparing different treatment onset times.

Estimates of effect size are shown with $95 \% \mathrm{Cls}$. Final value and change scores combined as mean differences. MD: mean difference; Cls: 95\% confidence intervals.

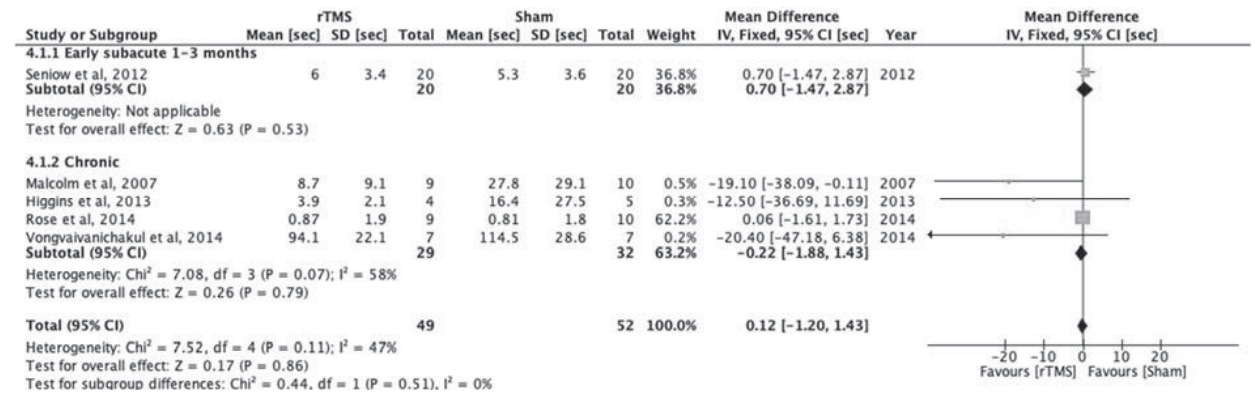

Figure S5.3.4: Effects of rTMS on the Wolf Motor Function Test in seconds (WMFT-sec) scale, comparing different treatment onset times.

Estimates of effect size are shown with 95\% Cls. MD: mean difference; Cls: 95\% confidence intervals. 


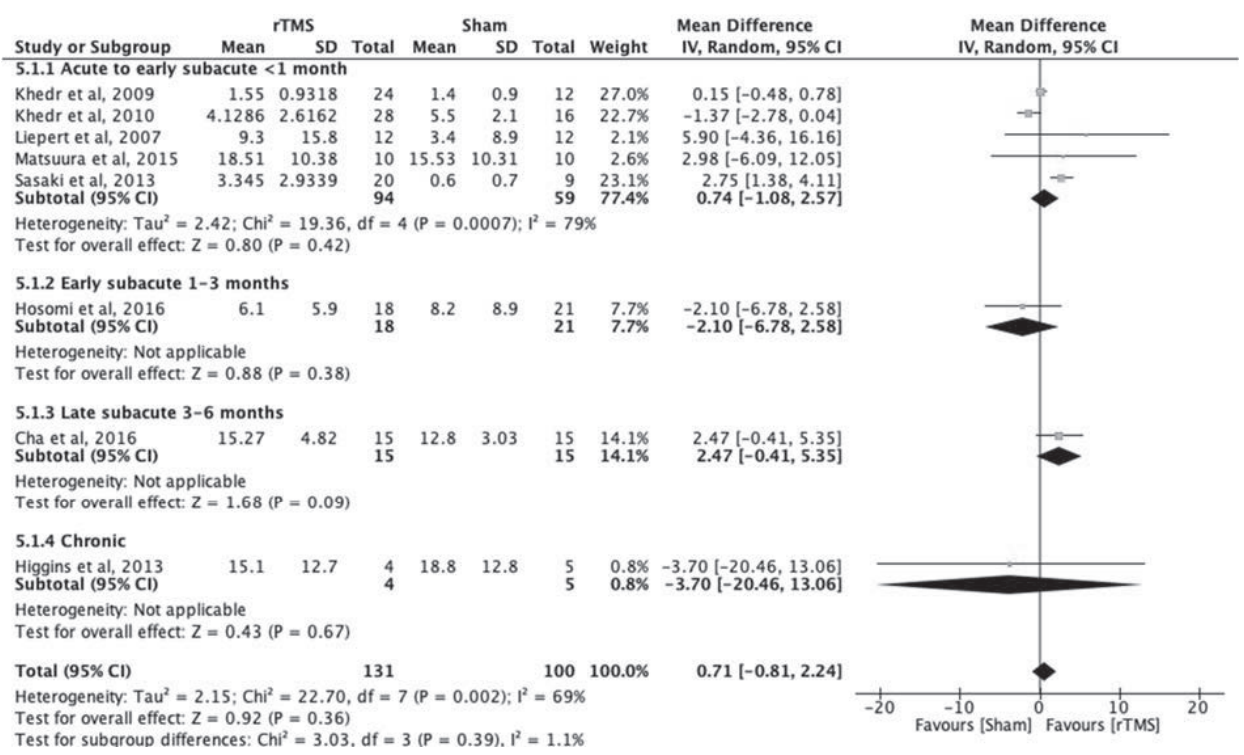

Figure S5.3.5: Effects of rTMS on the Grip Strength (GS) scale, comparing different treatment onset times.

Estimates of effect size are shown with 95\% Cls. MD: mean difference; Cls: 95\% confidence intervals.

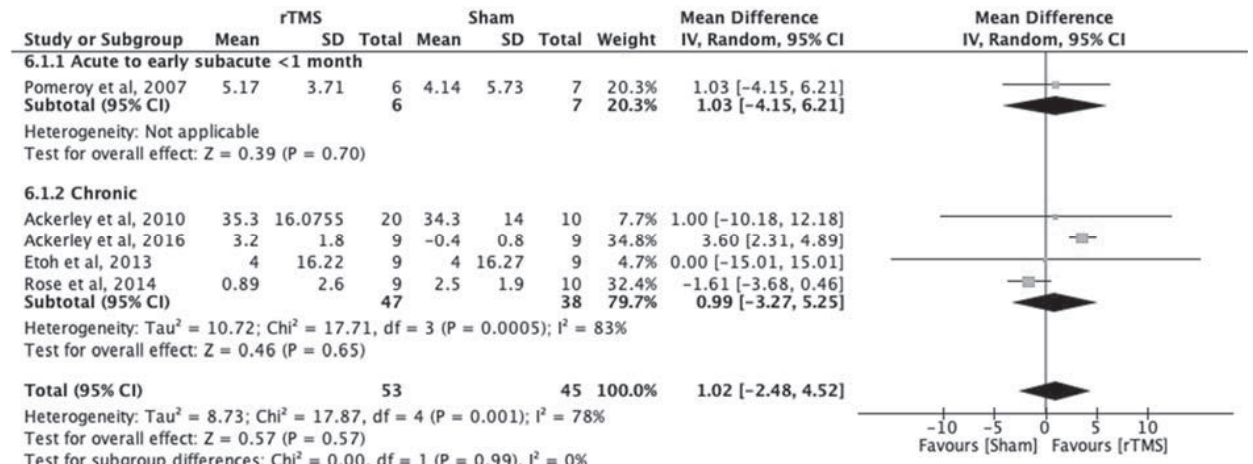

Figure S5.3.6: Effects of rTMS on the Action Research Arm Test (ARAT) scale, comparing different treatment onset times.

Estimates of effect size are shown with $95 \% \mathrm{Cls}$. MD: mean difference; $\mathrm{Cls}$ : 95\% confidence intervals. 


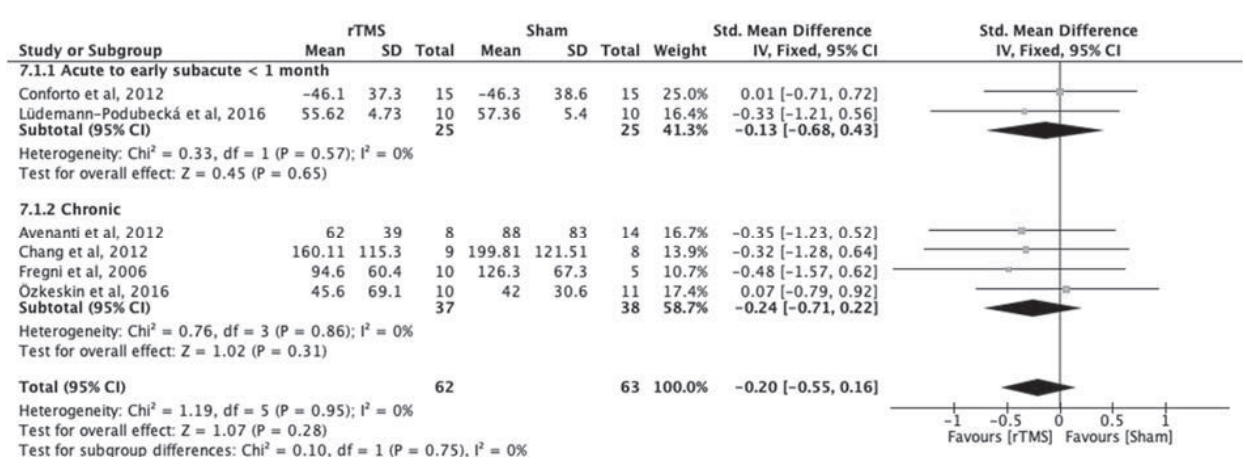

Figure S5.3.7: Effects of rTMS on the Jebsen Taylor Test (JTT) scale, comparing different treatment onset times.

Estimates of effect size are shown with 95\% Cls. SMD: standardized mean difference; Cls: 95\% confidence intervals.

\begin{tabular}{lrrrrrrrrrr} 
& \multicolumn{4}{c}{ rTMS } & \multicolumn{4}{c}{ Sham } & \multicolumn{3}{c}{ Std. Mean Difference } & Std. Mean Difference \\
IV, Random, 95\% CI
\end{tabular}

Figure S5.3.8: Effects of rTMS on the Pinch Force (PF) scale.

Estimates of effect size are shown with $95 \%$ Cls. SMD: standardized mean difference; Cls: $95 \%$ confidence intervals. 


\section{Appendix 5.4 Sensitivity analysis (omission of crossover and single-blind studies and studies without treatment allocation)}

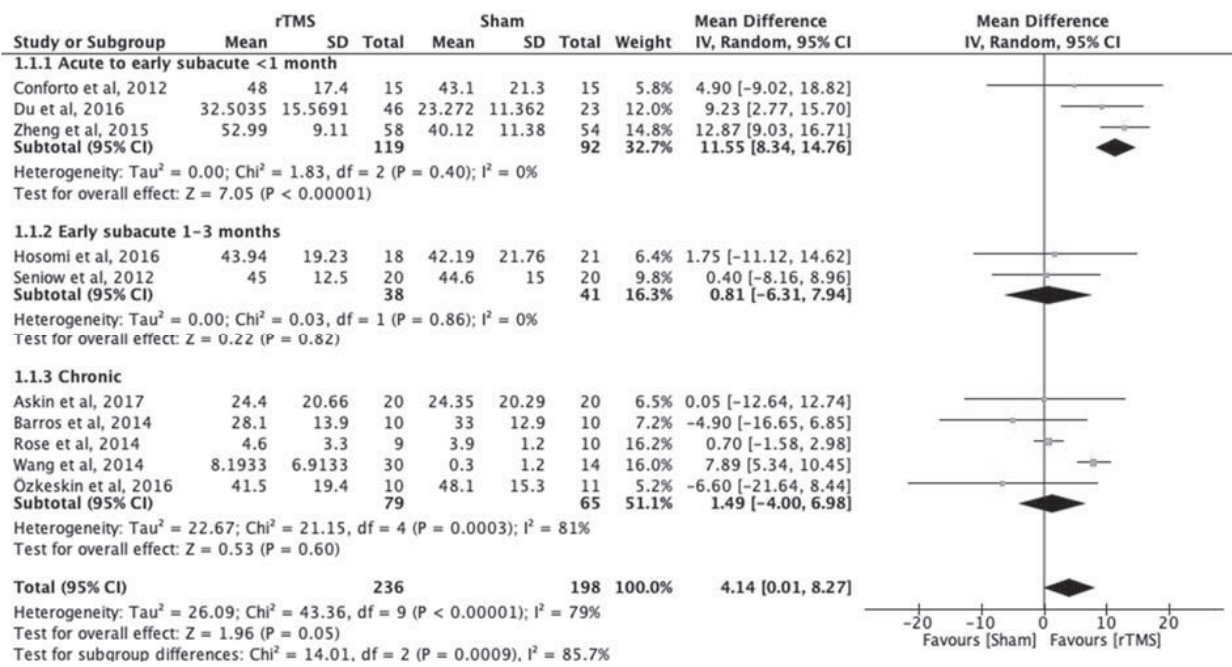

Figure S5.4.1: Effects of rTMS on the Fugl-Meyer Arm (FMA) scale without crossover, single-blind and no treatment allocation studies, comparing different treatment onset times.

Estimates of effect size are shown with $95 \% \mathrm{Cls}$. Final value and change scores combined as mean differences. MD: mean difference; Cls: 95\% confidence intervals.

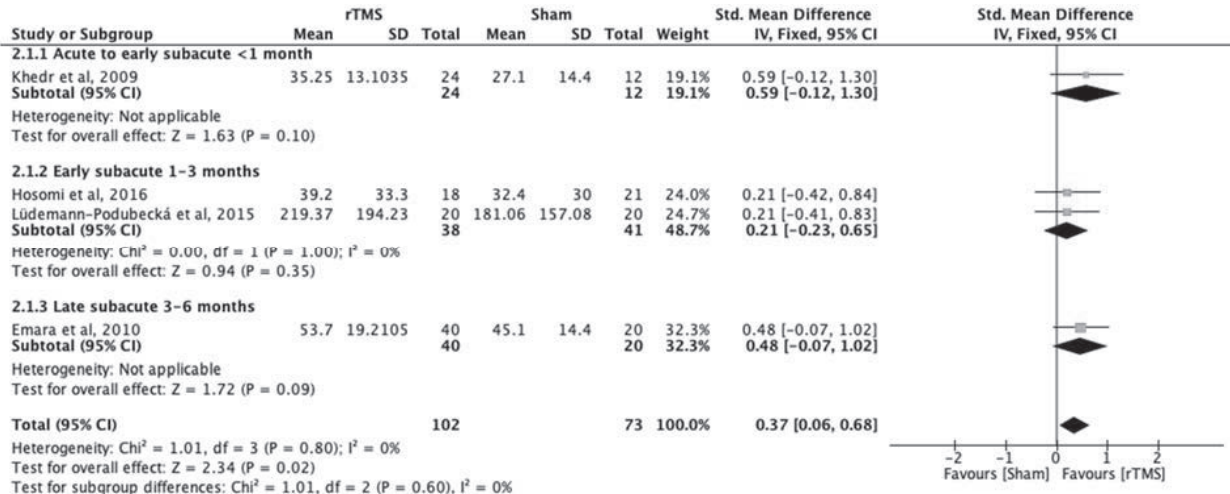

Figure S5.4.2: Effects of rTMS on the Finger Tapping (FT) scale without crossover, single-blind and no treatment allocation studies, comparing different treatment onset times.

Estimates of effect size are shown with $95 \% \mathrm{Cls}$. SMD: standardized mean difference; Cls: $95 \%$ confidence intervals. 


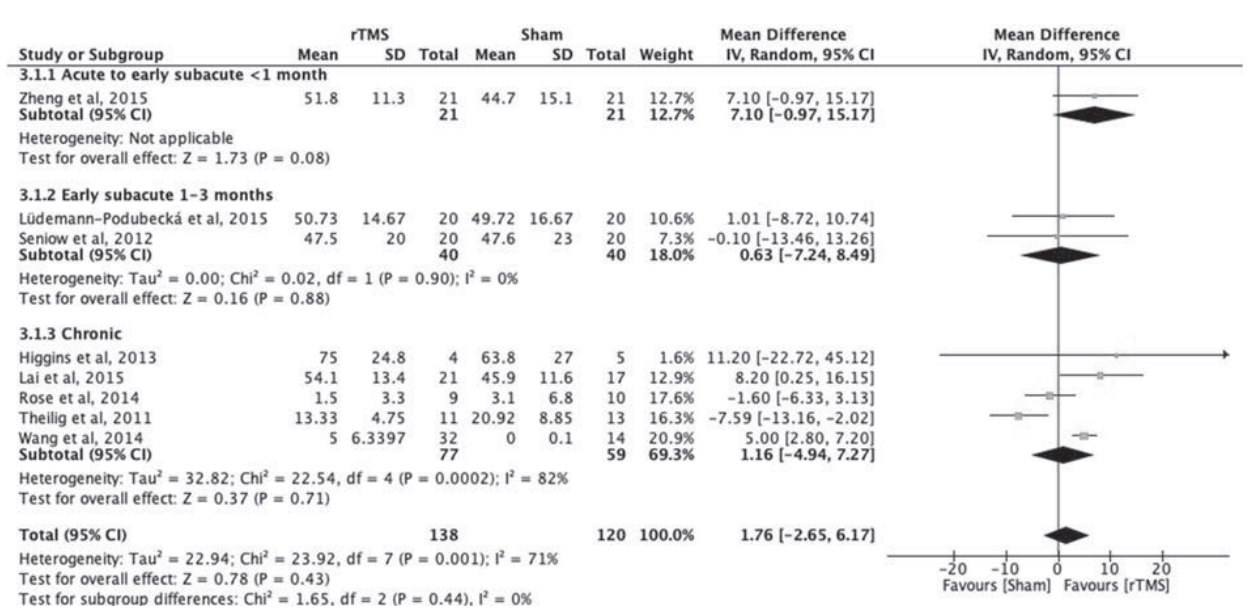

Figure S5.4.3: Effects of rTMS on the Wolf Motor Function Test (WMFT) scale without crossover, single-blind and no treatment allocation studies, comparing different treatment onset times.

Estimates of effect size are shown with $95 \% \mathrm{Cls}$. Final value and change scores combined as mean differences. MD: mean difference; Cls: 95\% confidence intervals.

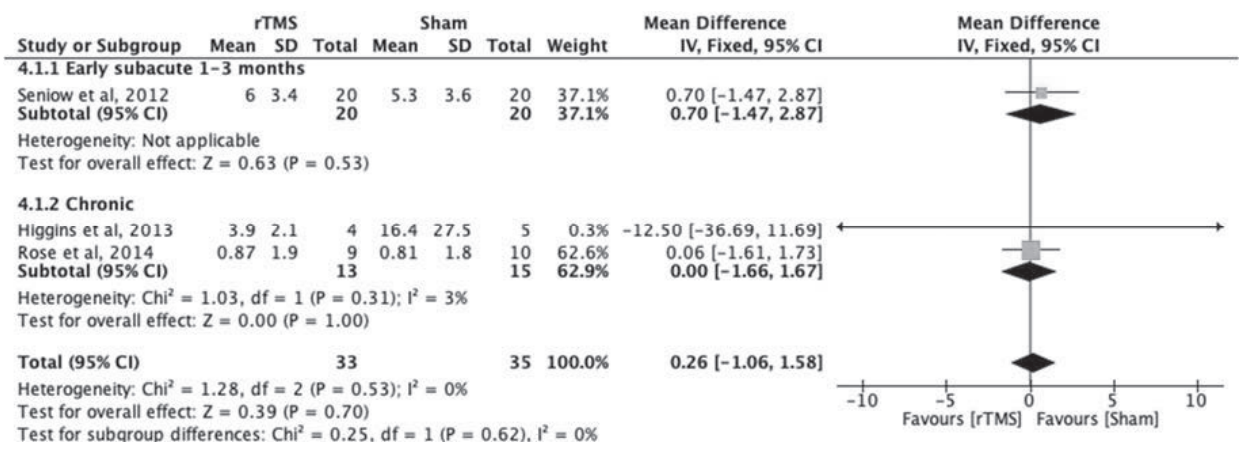

Figure S5.4.4: Effects of rTMS on the Wolf Motor Function Test in seconds (WMFT-sec) scale without single-blind and no treatment allocation studies, comparing different treatment onset times.

Estimates of effect size are shown with $95 \% \mathrm{Cls}$. Final value and change scores combined as mean differences. MD: mean difference; Cls: 95\% confidence intervals. 


\begin{tabular}{|c|c|c|c|c|c|c|c|c|c|}
\hline \multirow[b]{2}{*}{ Study or Subgroup } & \multicolumn{3}{|c|}{ Sham } & \multicolumn{3}{|c|}{ rTMS } & \multicolumn{2}{|r|}{ Mean Difference } & \multirow{2}{*}{$\begin{array}{l}\text { Mean Difference } \\
\text { IV, Fixed, } 95 \% \mathrm{CI}\end{array}$} \\
\hline & Mean & SD & Total & Mean & SD & Total & Weight & IV, Fixed, 95\% CI & \\
\hline Khedr et al, 2009 & 1.55 & 0.9318 & 24 & 1.4 & 0.9 & 12 & $78.9 \%$ & $0.15[-0.48,0.78]$ & \\
\hline \multicolumn{10}{|c|}{$\begin{array}{l}\text { Heterogeneity: Chi } i^{2}=3.71, d f=1(P=0.05) ; 1^{2}=73 \% \\
\text { Test for overall effect: } Z=0.35(P=0.73)\end{array}$} \\
\hline \multicolumn{10}{|c|}{$\begin{array}{l}\text { Heterogeneity: Not applicable } \\
\text { Test for overall effect: } Z=0.88(P=0.38)\end{array}$} \\
\hline \multicolumn{10}{|c|}{ 5.1.3 Late subacute $3-6$ months } \\
\hline $\begin{array}{l}\text { Cha et al, } 2016 \\
\text { Subtotal }(95 \% \mathrm{Cl})\end{array}$ & 15.27 & 4.82 & $\begin{array}{l}15 \\
15\end{array}$ & 12.8 & 3.03 & $\begin{array}{l}15 \\
15\end{array}$ & $\begin{array}{l}3.8 \% \\
3.8 \%\end{array}$ & $\begin{array}{l}2.47[-0.41,5.35] \\
2.47[-0.41,5.35]\end{array}$ & \\
\hline \multicolumn{10}{|l|}{ 5.1.4 Chronic } \\
\hline $\begin{array}{l}\text { Higgins et al, } 2013 \\
\text { Subtotal }(95 \% \mathrm{CI})\end{array}$ & 15.1 & 12.7 & $\begin{array}{l}4 \\
4\end{array}$ & 18.8 & 12.8 & $\begin{array}{l}5 \\
5\end{array}$ & $\begin{array}{l}0.1 \% \\
0.1 \%\end{array}$ & $\begin{array}{l}-3.70[-20.46,13.06] \\
-3.70[-20.46,13.06]\end{array}$ & \\
\hline \multicolumn{10}{|c|}{$\begin{array}{l}\text { Heterogeneity: Not applicable } \\
\text { Test for overall effect: } Z=0.43(P=0.67)\end{array}$} \\
\hline Total $(95 \% \mathrm{Cl})$ & & & 89 & & & 69 & $100.0 \%$ & $-0.04[-0.60,0.52]$ & 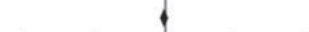 \\
\hline $\begin{array}{l}\text { Heterogeneity: } \mathrm{Chi}^{2}= \\
\text { Test for overall effect: } \\
\text { Test for subaroun dif }\end{array}$ & $\begin{array}{l}7.60, \mathrm{df}= \\
Z=0.13 \\
\text { erences: } C\end{array}$ & $\begin{array}{l}=4(P=C \\
(P=0.8 \\
C h i^{2}=3.8\end{array}$ & $\begin{array}{l}\text { 0.11); } l^{2} \\
\text { 9) } \mathrm{df}=\end{array}$ & ${ }^{2}=47 \%$ & & & & & 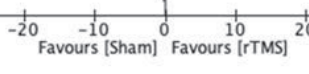 \\
\hline
\end{tabular}

Figure S5.4.5: Effects of rTMS on the Grip Strength (GS) scale without single-blind and no treatment allocation studies, comparing different treatment onset times.

Estimates of effect size are shown with $95 \% \mathrm{Cls}$. Final value and change scores combined as mean differences. MD: mean difference; Cls: $95 \%$ confidence intervals.

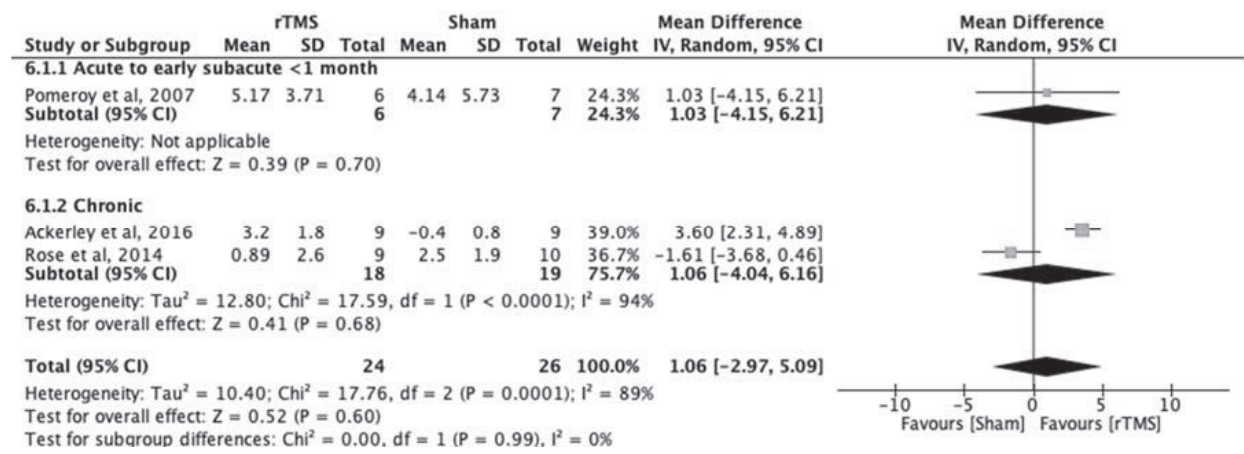

Figure S5.4.6: Effects of rTMS on the Action Research Arm Test (ARAT) scale without single-blind and no treatment allocation studies, comparing different treatment onset times.

Estimates of effect size are shown with $95 \% \mathrm{Cls}$. MD: mean difference; $\mathrm{Cls}$ : 95\% confidence intervals. 


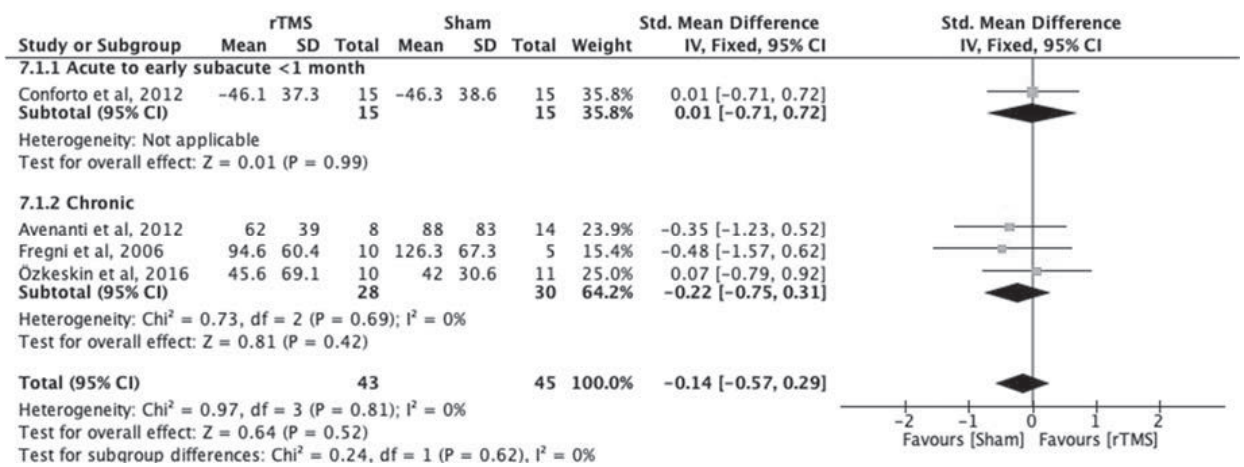

Figure S5.4.7: Effects of rTMS on the Jebsen Taylor Test (JTT) scale without single-blind and no treatment allocation studies, comparing different treatment onset times.

Estimates of effect size are shown with 95\% Cls. SMD: standardized mean difference; Cls: 95\% confidence intervals.

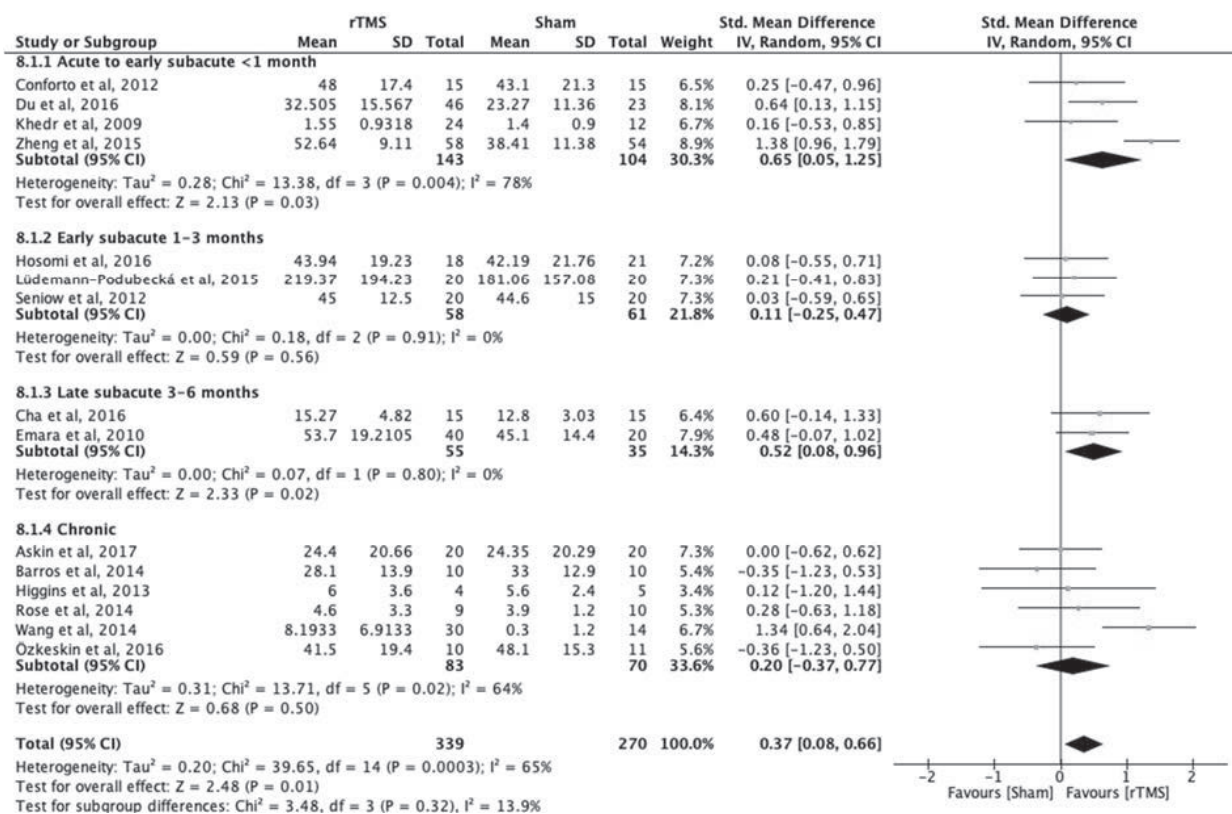

Figure S5.4.8: Effects of rTMS on the ICF Function domain without single-blind and no treatment allocation studies, comparing different treatment onset times.

Estimates of effect size are shown with 95\% Cls. SMD: standardized mean difference; Cls: 95\% confidence intervals. 


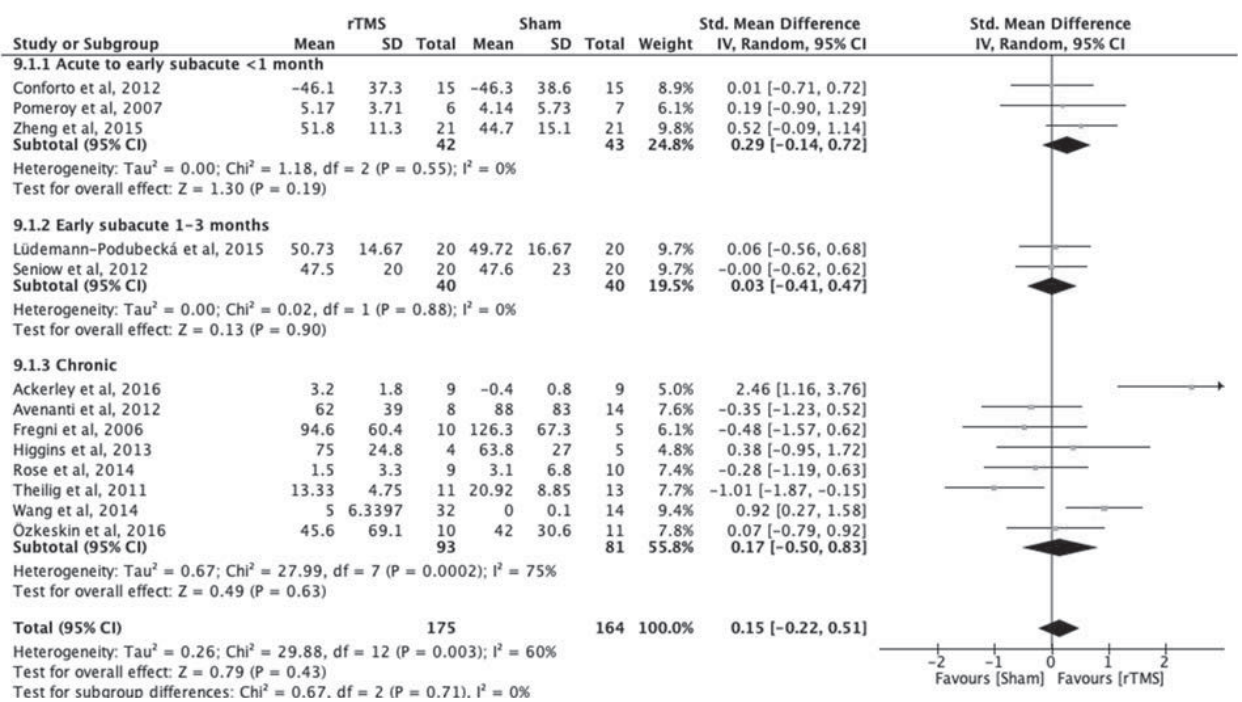

Figure S5.4.9: Effects of rTMS on the ICF Activity domain without single-blind and no treatment allocation studies, comparing different treatment onset times.

Estimates of effect size are shown with 95\% Cls. SMD: standardized mean difference; Cls: $95 \%$ confidence intervals. 


\section{Appendix 5.5 Funnel plot}

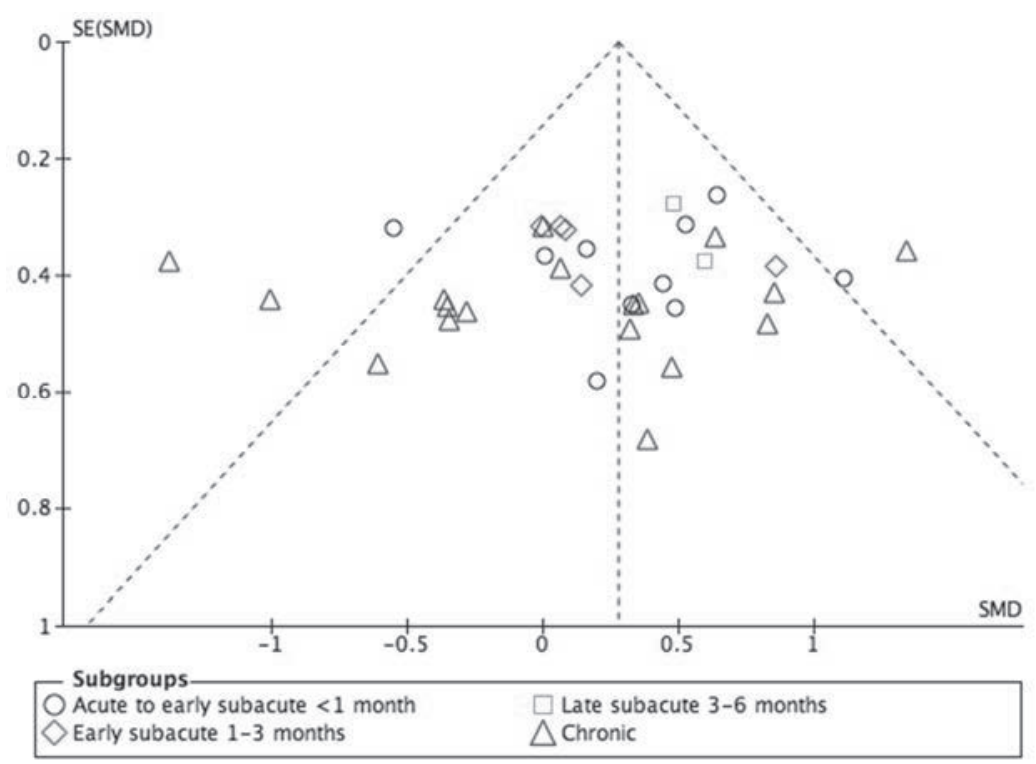




\section{Appendix 5.6 Additional subgroup analyses}

\section{A Number of treatment sessions}

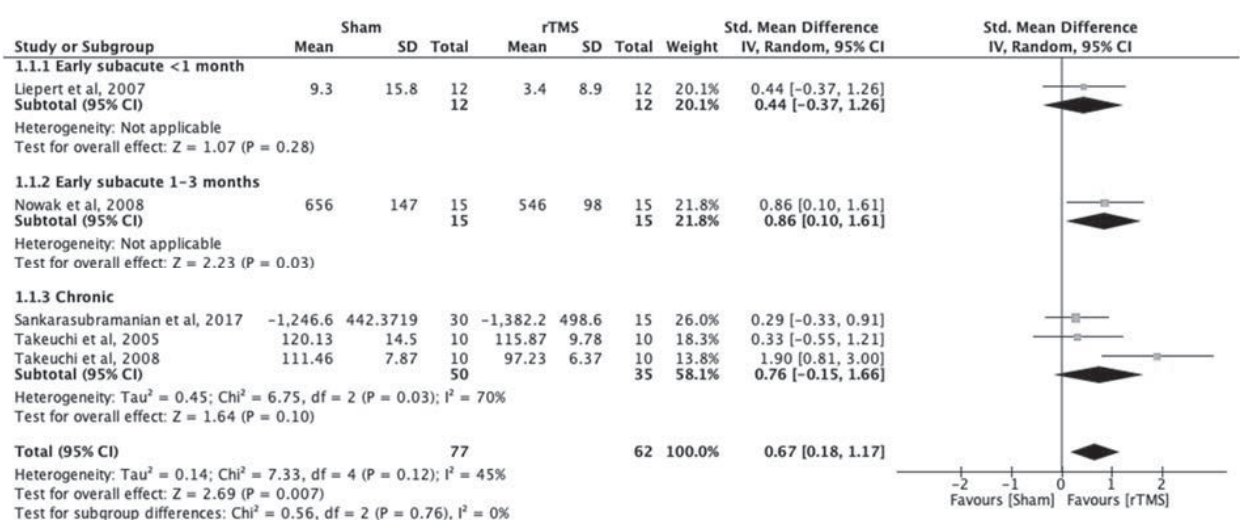

Figure S5.6.1: Effects of rTMS on the ICF Function domain with 1 treatment session, comparing different treatment onset times.

Estimates of effect size are shown with $95 \% \mathrm{Cls}$. SMD: standardized mean difference; Cls: $95 \%$ confidence intervals.

\begin{tabular}{|c|c|c|c|c|c|c|c|c|c|}
\hline Study or Subgroup & \multicolumn{3}{|c|}{ Sham } & \multicolumn{3}{|c|}{ rTMS } & \multicolumn{2}{|c|}{ Std. Mean Difference } & $\begin{array}{c}\text { Std. Mean Difference } \\
\text { IV, Fixed, } 95 \% \mathrm{CI}\end{array}$ \\
\hline $\begin{array}{l}\text { Ludemann-Podubecká et al, } 2016 \\
\text { Subtotal }(95 \% \text { CI) }\end{array}$ & 55.62 & 4.73 & $\begin{array}{l}10 \\
10\end{array}$ & 57.36 & 5.4 & $\begin{array}{l}10 \\
10\end{array}$ & $\begin{array}{l}33.3 \% \\
33.3 \%\end{array}$ & $\begin{array}{l}-0.33[-1.21,0.56] \\
-0.33[-1.21,0.56]\end{array}$ & \\
\hline \multicolumn{10}{|c|}{$\begin{array}{l}\text { Heterogeneity: Not applicable } \\
\text { Test for overall effect: } Z=0.73(P=0.47)\end{array}$} \\
\hline \multicolumn{10}{|l|}{ 2.1.2 Chronic } \\
\hline Ackerley et al, 2010 & 35.3 & 16.0755 & 20 & 34.3 & 14 & 10 & $45.1 \%$ & $0.06[-0.70,0.82]$ & \\
\hline \multirow{2}{*}{\multicolumn{4}{|c|}{$\begin{array}{l}\text { Heterogeneity: } \mathrm{Chi}^{2}=2.24, \mathrm{df}=2(\mathrm{P}=0.33) ; \mathrm{I}^{2}=11 \% \\
\text { Test for overall effect: } \mathrm{Z}=0.31(\mathrm{P}=0.76)\end{array}$}} & & & 27 & $100.0 \%$ & $0.08[-0.43,0.59]$ & \\
\hline & & & & $I^{2}=18.8$ & & & & & $\stackrel{-1}{-2} \stackrel{0}{0}{ }^{0}{ }^{2}$ \\
\hline
\end{tabular}

Figure S5.6.2: Effects of rTMS on the ICF Activity domain with 1 treatment session, comparing different treatment onset times.

Estimates of effect size are shown with 95\% Cls. SMD: standardized mean difference; Cls: 95\% confidence intervals. 


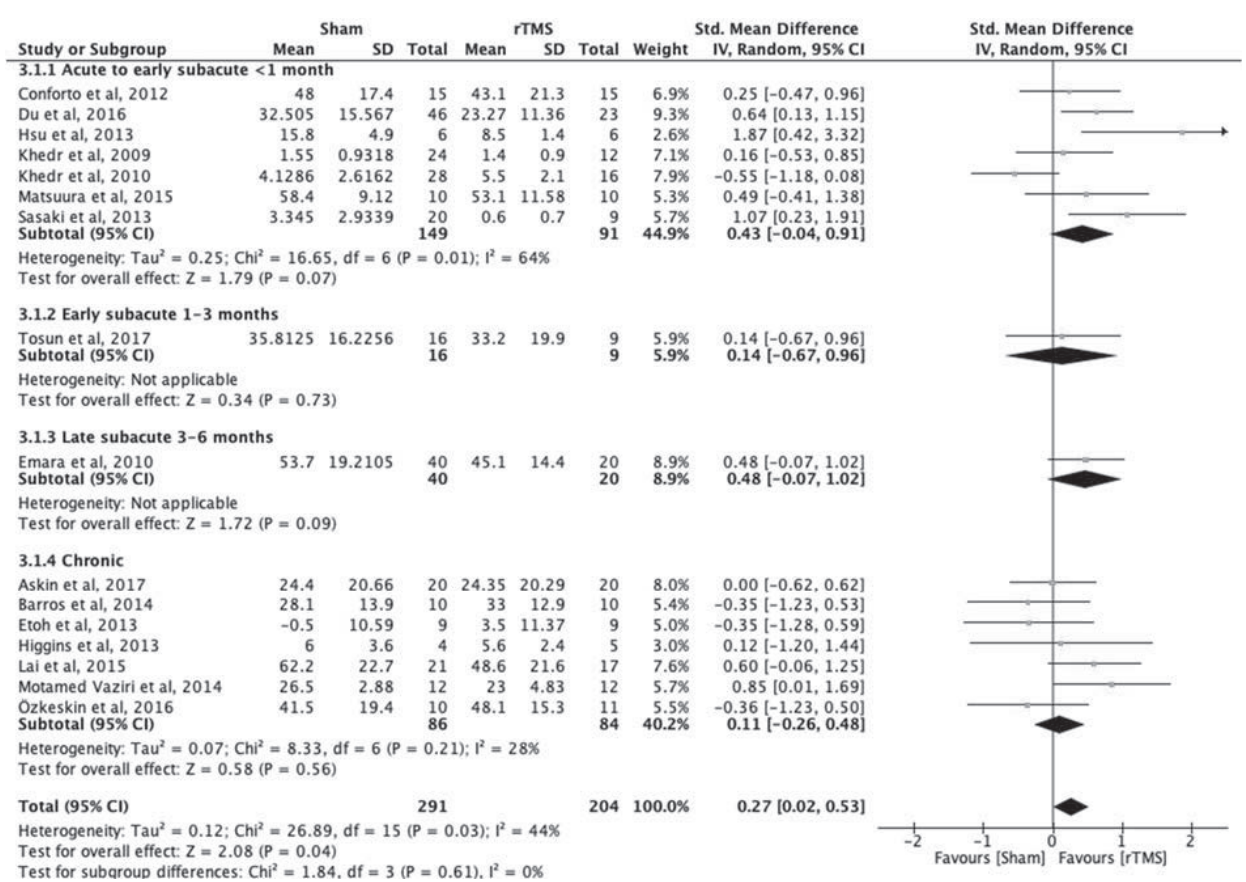

Figure S5.6.3: Effects of rTMS on the ICF Function domain with 2-10 treatment sessions, comparing different treatment onset times.

Estimates of effect size are shown with 95\% Cls. SMD: standardized mean difference; Cls: 95\% confidence intervals.

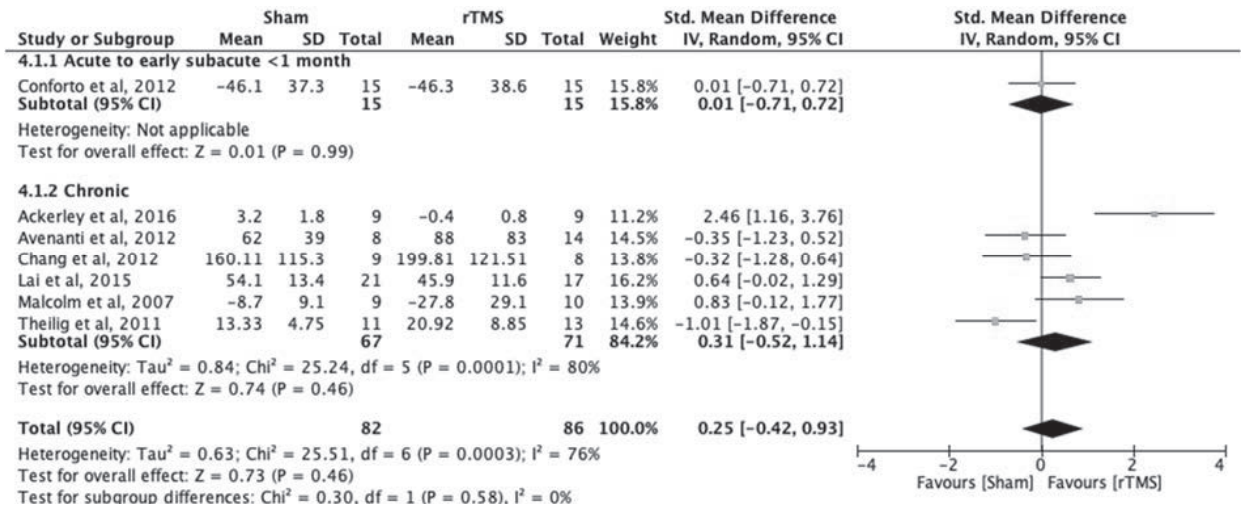

Figure S5.6.4: Effects of rTMS on the ICF Activity domain with 2-10 treatment sessions, comparing different treatment onset times.

Estimates of effect size are shown with 95\% Cls. SMD: standardized mean difference; Cls: $95 \%$ confidence intervals. 


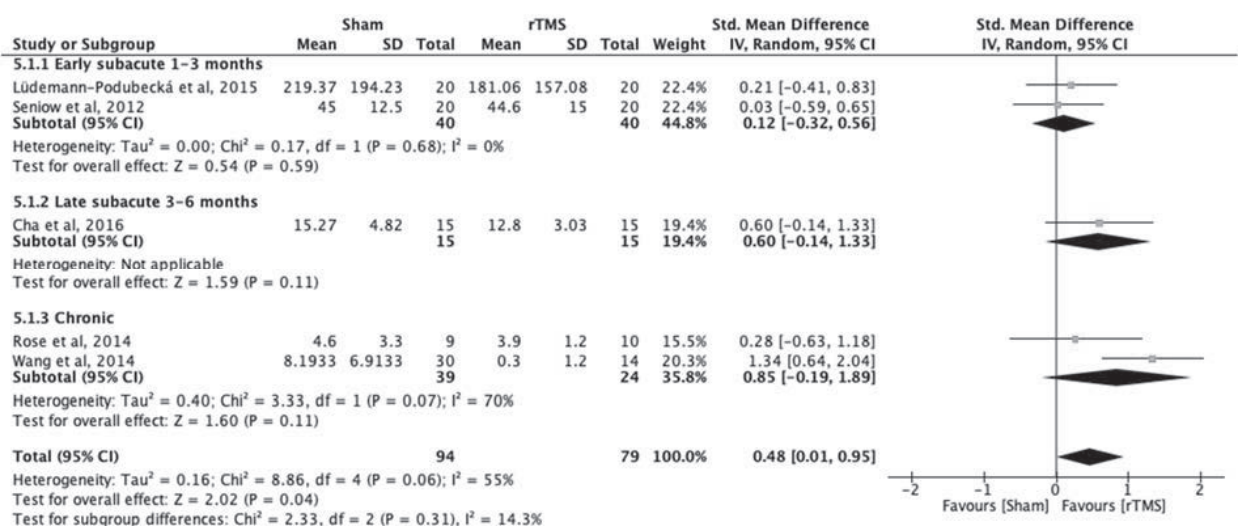

Figure S5.6.5: Effects of rTMS on the ICF Function domain with 11-20 treatment sessions, comparing different treatment onset times.

Estimates of effect size are shown with $95 \% \mathrm{Cls}$. SMD: standardized mean difference; Cls: $95 \%$ confidence intervals.

\section{B Additional therapy}

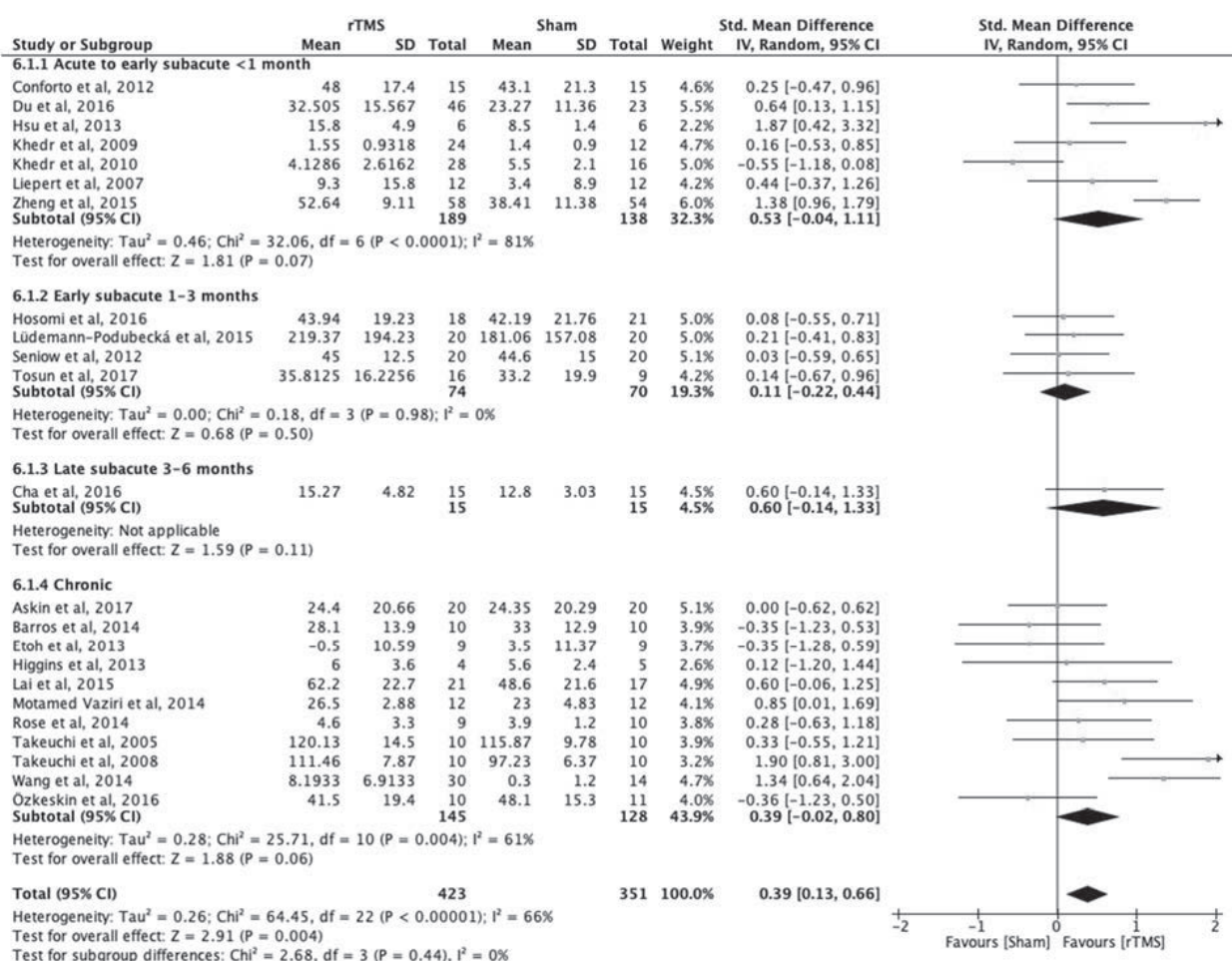

Figure S5.6.6: Effects of rTMS on the ICF Function domain with additional therapy, comparing different treatment onset times.

Estimates of effect size are shown with $95 \% \mathrm{Cls}$. SMD: standardized mean difference; Cls: $95 \%$ confidence intervals. 


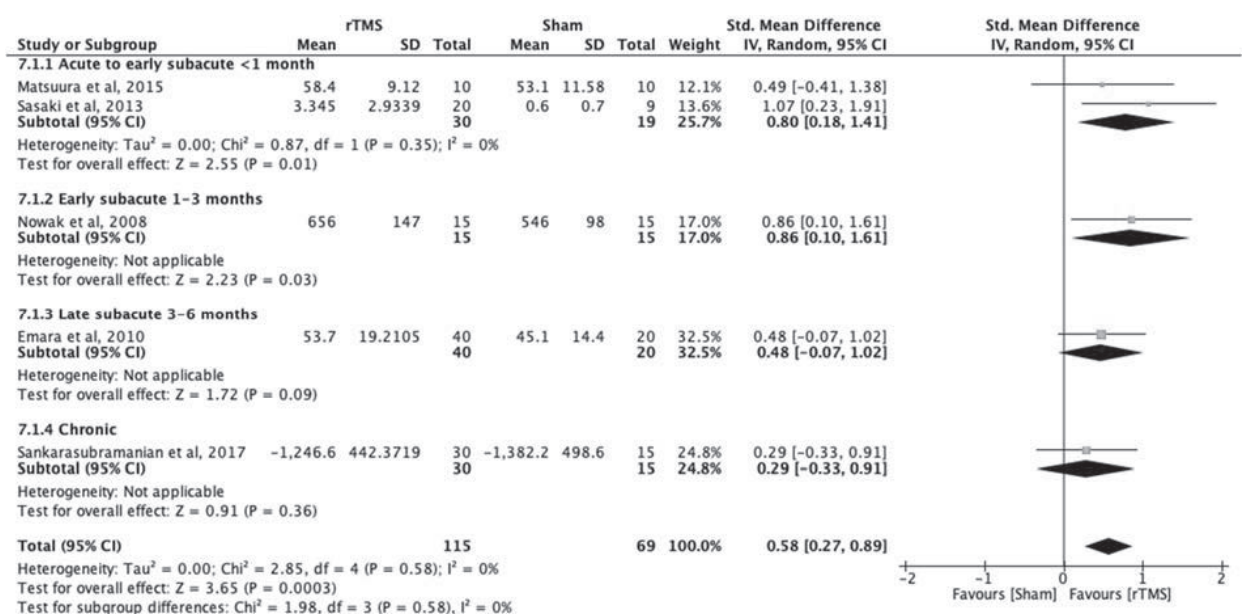

Figure S5.6.7: Effects of rTMS on the ICF Function domain without additional therapy, comparing different treatment onset times.

Estimates of effect size are shown with 95\% Cls. SMD: standardized mean difference; Cls: 95\% confidence intervals.

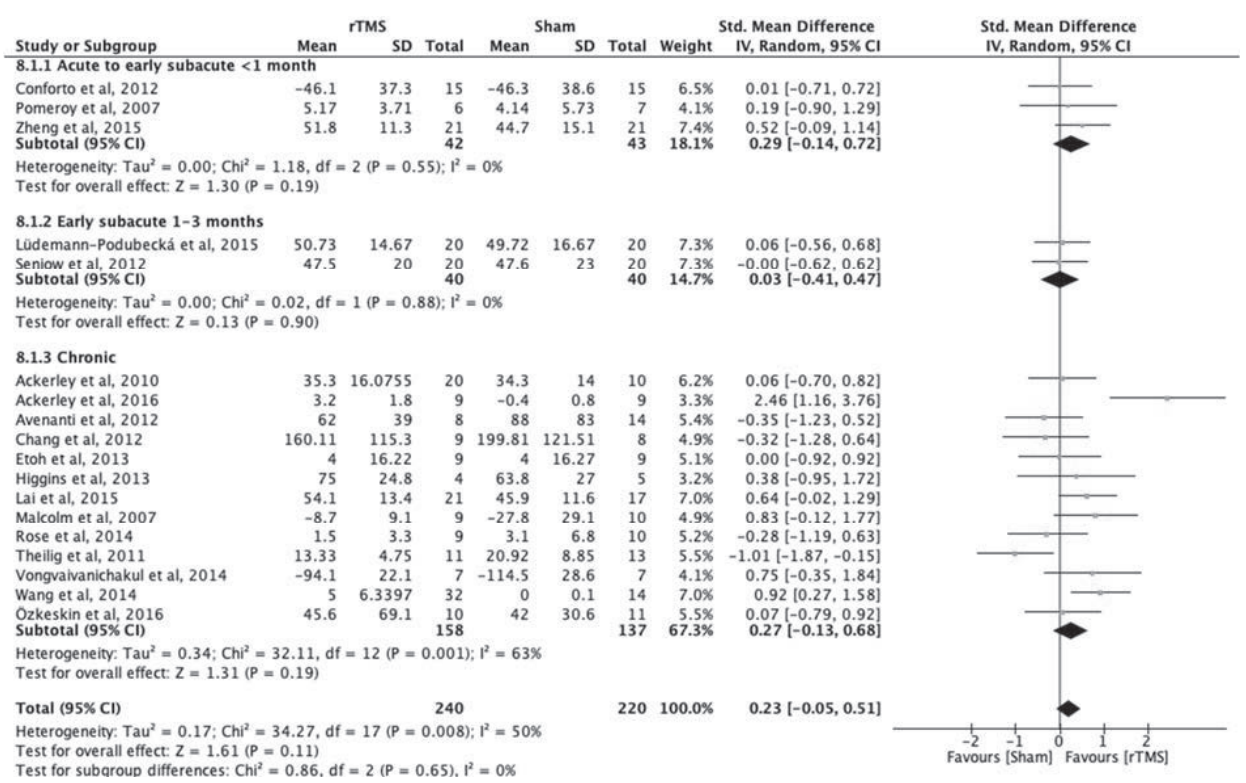

Figure S5.6.8: Effects of rTMS on the ICF Activity domain with additional therapy, comparing different treatment onset times.

Estimates of effect size are shown with $95 \%$ Cls. SMD: standardized mean difference; Cls: $95 \%$ confidence intervals. 


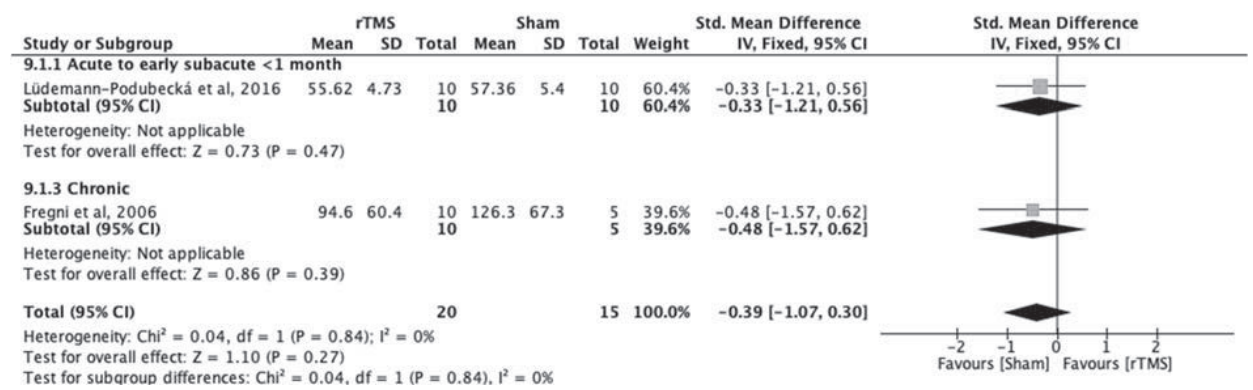

Figure S5.6.9: Effects of rTMS on the ICF Activity domain without additional therapy, comparing different treatment onset times.

Estimates of effect size are shown with 95\% Cls. SMD: standardized mean difference; Cls: 95\% confidence intervals.

\section{C rTMS frequency/site of stimulation}

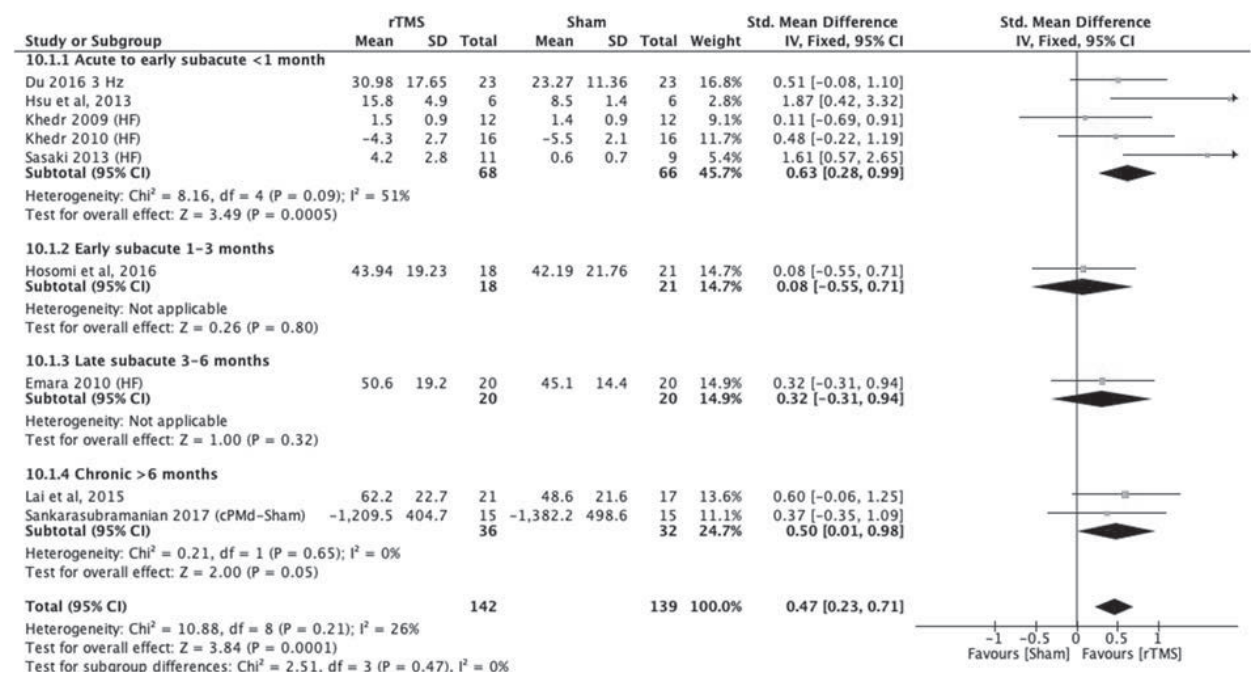

Figure S5.6.10: Effects of high frequency rTMS to the affected hemisphere on the ICF Function domain, comparing different treatment onset times.

Estimates of effect size are shown with $95 \% \mathrm{Cls}$. SMD: standardized mean difference; Cls: $95 \%$ confidence intervals. 


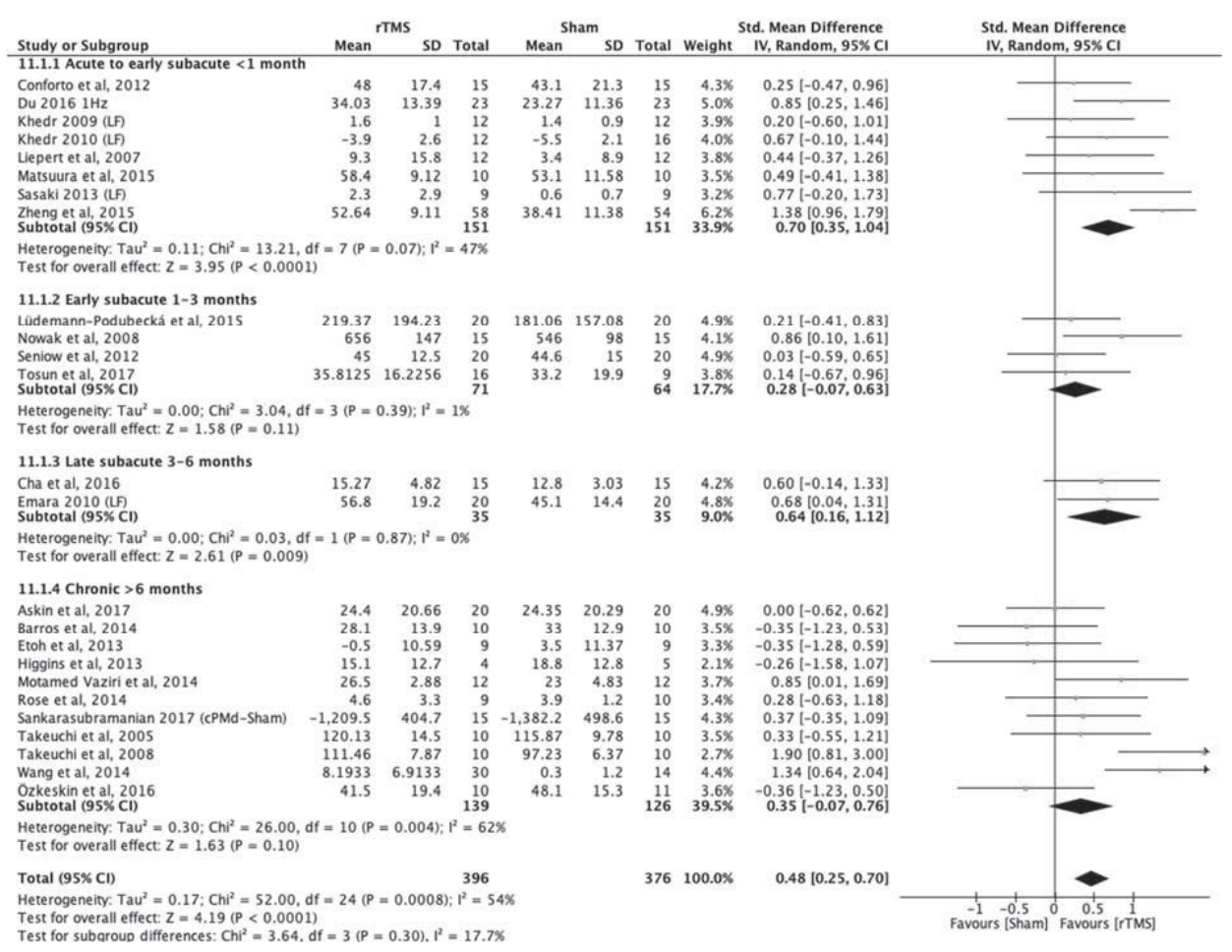

Figure S5.6.11: Effects of low frequency rTMS to the unaffected hemisphere on the ICF Function domain, comparing different treatment onset times.

Estimates of effect size are shown with 95\% Cls. SMD: standardized mean difference; Cls: 95\% confidence intervals. 


\section{Brain stimulation for arm recovery after stroke (B-STARS): Protocol for a randomized controlled trial in subacute stroke patients}

Eline C.C. van Lieshout Johanna M.A. Visser-Meily Sebastiaan F.W. Neggers

H. Bart van der Worp Rick M. Dijkhuizen 


\section{Abstract}

Introduction: Many stroke patients have moderate to severe long-term sensorimotor impairments, often including inability to execute movements of the affected arm or hand. Limited recovery from stroke may be partly caused by imbalanced interaction between the cerebral hemispheres, with reduced excitability of the ipsilesional motor cortex while excitability of the contralesional motor cortex is increased. Non-invasive brain stimulation with inhibitory repetitive transcranial magnetic stimulation (rTMS) of the contralesional hemisphere may aid in relieving a post-stroke interhemispheric excitability imbalance, which could improve functional recovery. There are encouraging effects of theta burst stimulation (TBS), a form of TMS, in patients with chronic stroke, but evidence on efficacy and long-term effects on arm function of contralesional TBS in subacute hemiparetic stroke patients is lacking.

Methods and analysis: In a randomized clinical trial, we will assign 60 patients with a firstever ischemic stroke in the previous 7-14 days and a persistent paresis of one arm to 10 sessions of real stimulation with TBS of the contralesional primary motor cortex or to sham stimulation over a period of 2 weeks. Both types of stimulation will be followed by upper limb training. A subset of patients will undergo five MRI sessions to assess post-stroke brain reorganisation. The primary outcome measure will be the upper limb function score, assessed from grasp, grip, pinch and gross movements in the action research arm test, measured at 3 months after stroke. Patients will be blinded to treatment allocation. The primary outcome at three months will also be assessed in a blinded fashion.

Ethics and dissemination: The study has been approved by the Medical Research Ethics Committee of the University Medical Center Utrecht, The Netherlands. The results will be disseminated through (open access) peer-reviewed publications, networks of scientists, professionals and the public, and presented at conferences.

Trial registration number: NTR6133.

\section{Strengths and limitations of this study}

- To the best of our knowledge, this is one of the first clinical trials evaluating the effect of theta burst stimulation on motor function in patients with subacute stroke (within 2 weeks);

- Long-term follow-up period up to 1 year after stroke onset, enabling assessment of durability of treatment effects;

- Multiple, different outcome measures, including motor function, activities of daily living, and neural network activity;

- Participants are blinded to their allocated treatment group throughout the trial, but study personnel are only blinded for the primary outcome measurement at 3 months. 


\section{Introduction}

Each year > 15 million people worldwide have a stroke, which is a major cause of adult disability. The most common functional deficits after stroke are sensorimotor impairments, which, in addition to functional disability, can have considerable negative effects on quality of life and societal participation. ${ }^{1-5}$ In 33-66\% of stroke patients with a paretic arm, recovery of arm function is absent or negligible in the first 6 months, ${ }^{6-8}$ and $>50 \%$ do not show signs of significant arm function recovery after $>5$ years post-stroke. ${ }^{9-12}$ On the other hand, 5-20\% of patients with impaired arm function after stroke demonstrate full functional recovery of arm function within 6 months. ${ }^{6,13}$ Rehabilitation programs contribute to functional recovery, and significant improvements in sensorimotor function can be achieved. ${ }^{7,14}$ Several studies suggest that functional improvement after stroke may be augmented by strategies that involve neuromodulation through non-invasive brain stimulation, such as transcranial magnetic stimulation (TMS), in combination with rehabilitative training. ${ }^{15-21}$

TMS provides a nonsinvasive and safe way to directly facilitate or suppress brain activity in cortical areas. TMS induces a current in the cerebral cortex through a coil that generates a magnetic field. ${ }^{22}$ Repetitive delivery of TMS (rTMS) with a high-frequency train of pulses is believed to enhance cortical excitability, while repetitive low-frequency TMS would suppress cortical excitability. ${ }^{22,23}$ TMS-induced reduction of cortical excitability has been associated with long-term depression (LTD)-like effects whereas TMS-induced increase of corticospinal excitability has been associated with long-term potentiation (LTP)-like effects. ${ }^{24}$ Recently, patterned protocols consisting of short trains of high-frequency TMS $(30-100 \mathrm{~Hz})$ alternating with rest periods in the theta-frequency range $(4-7 \mathrm{~Hz}$ ) (theta-burst stimulation $(\mathrm{TBS}))^{23,25}$ have been reported to provide effective and reliable paradigms for excitatory (intermittent TBS (iTBS)) or inhibitory (continuous TBS (cTBS)) brain stimulation. ${ }^{26}$ Three pulses of stimulation are delivered at $50 \mathrm{~Hz}$, given every $200 \mathrm{~ms}$. TBS paradigms are particularly promising because stimulation sessions are shorter (2-3 minutes or less) (i.e. more practical) as compared with standard rTMS protocols (20-30 minutes). ${ }^{27-30}$

Patients with hemiparetic stroke often have a functionally imbalanced interaction between the damaged and undamaged brain hemispheres, with reduced excitability of the ipsilesional motor cortex while excitability in the contralesional motor cortex is increased. ${ }^{31-38}$ Recent proof-of-principle studies have demonstrated that specific TMS paradigms - that is, facilitatory stimulation of the affected hemisphere to upregulate excitability, or inhibitory stimulation of the unaffected hemisphere to downregulate excitability - can elicit significant behavioural improvement in recovering patients with stroke. ${ }^{15,20,39}$ cTBS of the intact contralesional primary motor cortex may offer the most straightforward approach, as this brain region is easily identified from single-pulse TMS-induced motor evoked potentials 
(MEPs), which is more complicated in the structurally and/or functionally injured ipsilesional motor cortex. ${ }^{40,41}$

The feasibility and safety of TBS in patients with hemiparetic stroke have been demonstrated in a number of studies. ${ }^{27-30,42,43}$ However, most earlier studies involved chronic patients in whom post-stroke neural network reorganization had probably stabilized already, which may constrain the therapeutic potential of TBS. The most recent of these studies was a randomized clinical trial $(\mathrm{RCT})$ in which chronic subcortical stroke patients were treated with iTBS of the ipsilesional motor cortex directly followed by upper limb physical therapy, daily for 10 days. Upper limb function, assessed with the action research arm test (ARAT), improved for at least 1 month after treatment compared to sham therapy. ${ }^{43}$ Until now, however, a RCT on the long-term effects of TBS treatment in patients with subacute hemiparetic stroke is lacking. Previous studies that tested TMS treatment in the subacute phase after stroke applied low frequency or high frequency rTMS, some had a small sample size (18-58 patients) or was not supported by a power calculation and relatively short intervention duration of 5-10 days. ${ }^{39,44-48}$ A RCT on the long-term effects of repetitive TBS in a larger patient population subacutely after stroke would provide important new insights into the therapeutic efficacy of this non-invasive and practicable intervention during an optimal time-window for neurorehabilitation, especially in combination with a pragmatic upper limb training approach directly following brain stimulation.

\section{Objectives}

The primary objective of this study is to determine the therapeutic effect of contralesional cTBS (inhibitory stimulation) on recovery of function of the paretic arm at 3 months after ischaemic stroke. The secondary objectives are to evaluate:

1) the mode of action of contralesional cTBS on neural network reorganisation after ischemic stroke, at different time-points;

2) the therapeutic effect of contralesional cTBS on other sensorimotor functions, at different time-points post-treatment and

3) the therapeutic effect of contralesional cTBS on disability and quality of life, at different time-points post-treatment. 


\section{Methods and analysis}

\section{Study design ${ }^{1}$}

The Brain-STimulation for Arm Recovery after Stroke (B-STARS) study is a prospective, randomized, double-blind, controlled clinical trial. Subjects will be randomly allocated to real or sham brain stimulation, followed by standard care upper limb training. They will remain blinded to treatment allocation. Non-invasive brain stimulation will involve 10 daily sessions of cTBS of the contralesional hand area of the primary motor cortex over a period of 2 weeks. The first cTBS session will be executed within 2 weeks after stroke onset. Patients will be tested seven times in total (Figure 6.1): at the start of the study (T0; baseline), at the 10th day of the intervention (T1), and at 1 week (T2) and 1 month (T3) after stimulation,

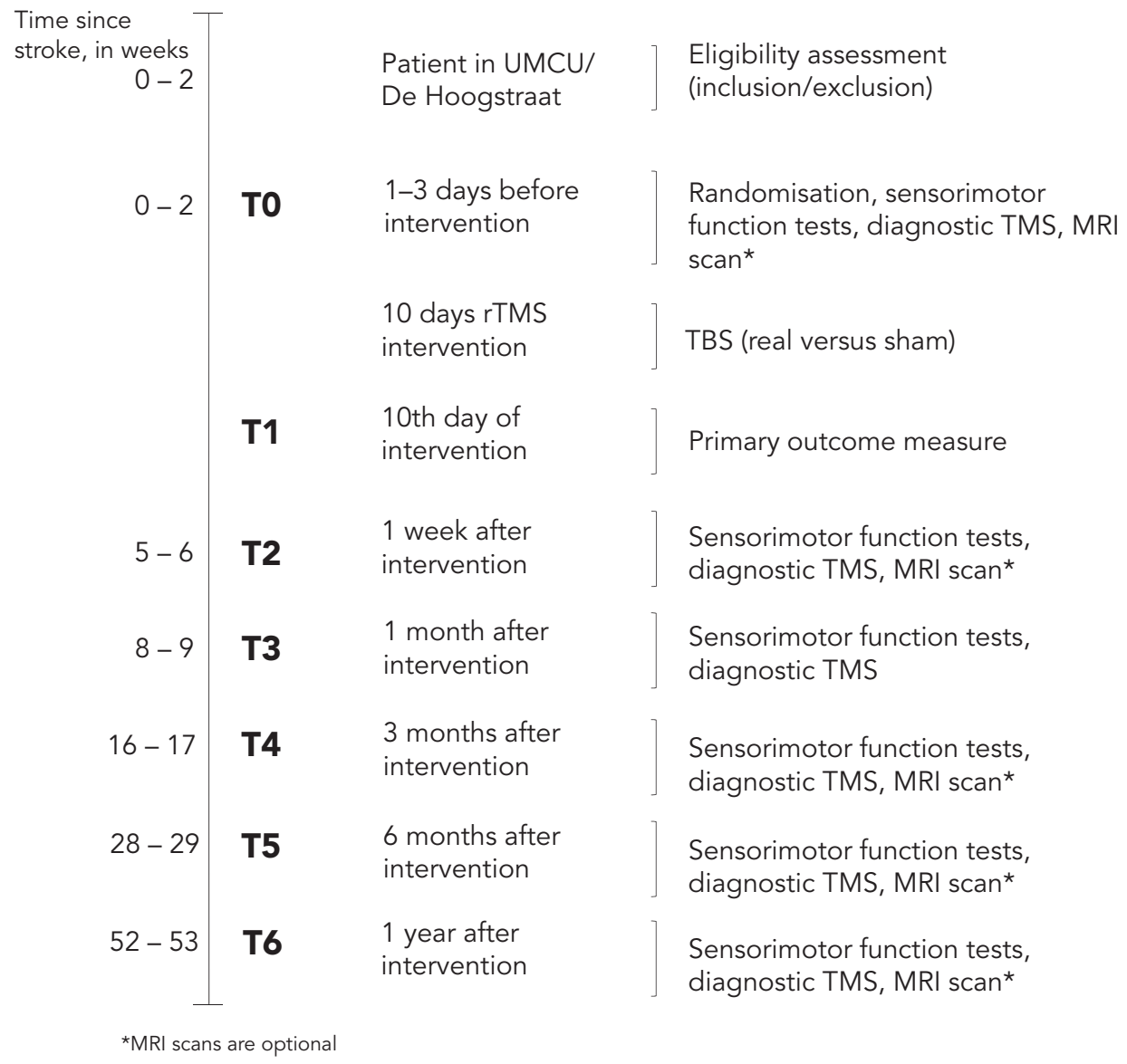

Figure 6.1: Schematic overview of the study procedure.

See important amendments in Appendix 6.1. 
and at 3 months (T4), 6 months (T5) and 1 year after stroke onset (T6). All baseline and follow-up assessments, except for the primary outcome measurement, will be conducted by a trained researcher (ECCVL) with support from research assistants.

The study has been approved by the Medical Research Ethics Committee of the University Medical Center Utrecht.

We used the SPIRIT (Standard Protocol Items: Recommendations for Interventional Trials) recommendations on reporting.

\section{Study population}

Participants (total of 60) will be recruited from the University Medical Center Utrecht and rehabilitation center De Hoogstraat in Utrecht, The Netherlands. Patients who fulfil the study criteria will be asked to participate, and they will receive a Patient Information Letter explaining the background and methods of the study. Patients can decide to participate with or without additional MRI scanning at the University Medical Center Utrecht. After written informed consent, patients will be randomly allocated to the treatment procedures.

\section{Inclusion and exclusion criteria}

The inclusion criteria of this study are: (1) patient age $\geq 18$ years; (2) first-ever unilateral ischaemic stroke; (3) paresis of one arm, with Shoulder Abduction and Finger Extension scores for shoulder abduction of $\geq 9$ on the Motricity Index and for finger extension of $\geq 1$ on the Fugl-Meyer score;49 (4) admission to 'De Hoogstraat' within the first 2 weeks after stroke onset; and (5) written informed consent. The exclusion criteria are: (1) disabling medical history (severe or recent heart disease, severe head trauma, coercively treated at a psychiatric ward); (2) history of epilepsy; (3) normal to almost normal use of hand, with a Motricity Index hand score of 33 (maximum score for this item, reflecting 'normal' function); (4) severe deficits in communication, memory or understanding that impede proper study participation, as determined by the treating physician, and (5) contraindications for TMS (metal (implants) in skull/scalp/head or fragments from welding or metalwork, implanted device (e.g. spinal cord stimulator, cardiac pacemaker, cochlear implants), pregnancy, and so on)..$^{50}$

\section{Randomisation}

Patients will be randomly assigned to either the real cTBS or sham cTBS group, stratified to the severity of their arm paresis. Patients in the high-performance group must demonstrate a minimal ability of finger extension of the thumb or one or more fingers, while patients in 
the low-performance group have no ability of finger extension of the thumb or one or more fingers. ${ }^{49}$ Randomisation will be performed with a secured electronic data capture system (Research Online 2.0, Julius Centrum). This will be done after the baseline assessment to account for possible improvements in motor function during the first days after stroke.

\section{Intervention}

All patients will receive the current standard rehabilitation program parallel to the treatment. The rehabilitation program consists of daily group treatment focused on the arm/hand (on functional and activity level, ca. 60 minutes), next to individual physical therapy, occupational therapy, creative therapy, speech therapy, and so on. All therapists and other rehabilitation staff will be blinded to group allocation. In addition, real cTBS or sham cTBS in combination with upper limb training will be applied in daily sessions during 2 weeks (10 working days), starting within 7-14 days after stroke onset. TBS will be performed using a NeuroMS/D Advanced Therapeutic stimulator (Neurosoft, Russia). cTBS will only be executed at rehabilitation center De Hoogstraat. We will employ a standard cTBS paradigm consisting of three stimuli bursts at $50 \mathrm{~Hz}$, repeated at $5 \mathrm{~Hz}$ frequency, resulting in 600 stimuli in 40 s. ${ }^{25}$ cTBS intensity will be at $70 \%$ of resting motor threshold (RMT), which induces highly consistent LTD-like MEP suppression with low intersubject variability. ${ }^{51}$ Sham stimulation will be done with the stimulator in sham mode (generates pulses at $90 \%$ lower intensity of RMT), with the coil oriented at an angle of $45^{\circ}$ relative to the scalp. ${ }^{20}$ For each session, the resting motor threshold will be determined from electromyography (EMG) (recorded with two Ag/ $\mathrm{AgCl}$ surface electrodes) from the contralateral first dorsal interosseous (FDI) muscle. The motor threshold will be defined as the minimum intensity of TMS over the hand area of the contralesional primary motor cortex to elicit at least five contralateral MEPs with $>50 \mu \mathrm{V}$ peak-to-peak amplitude in 10 trials with 7s intertrial intervals. A neuronavigation system (Brain Science Tools, the Netherlands), using a pre-stimulation CT or MRI scan (both techniques have been successfully applied for stereotactic imaging ${ }^{51,52}$ ) will be used to ensure consistent coil placement for cTBS of the hand area of the primary motor cortex. Applied protocol(s) will be in accordance with most recent safety and tolerability guidelines for TMS applications. ${ }^{26,50,53}$

\section{Outcome measures}

\section{Primary outcome measure}

The primary outcome measure will be the change in the modified ARAT score ${ }^{54}$ assessed at 3 months post stroke (see Table 6.1 for all times of testing). The ARAT is a performance test which assesses the ability to perform gross movements and the ability to grasp, move and release objects differing in size, weight and shape. The original test consists of 19 items, 
Table 6.1: Overview of functional outcome measures (motor function tests and questionnaires; including timing)

\begin{tabular}{|c|c|c|c|c|c|c|c|c|}
\hline & Instrument & TO & $\mathrm{T} 1$ & T2 & T3 & $\mathrm{T} 4$ & T5 & T6 \\
\hline \multicolumn{9}{|l|}{ Motor function } \\
\hline & $\begin{array}{l}\text { Action Research Arm Test } \\
\text { (ARAT) (primary outcome } \\
\text { measure) }\end{array}$ & $x$ & $x$ & $x$ & $x$ & $x$ & $x$ & $x$ \\
\hline & Fugl-Meyer (FM) & $x$ & & $x$ & $x$ & $x$ & $x$ & $x$ \\
\hline & $\begin{array}{l}\text { Stroke Upper Limb } \\
\text { Capacity Scale (SULCS) }\end{array}$ & $x$ & & & $x$ & $x$ & $x$ & \\
\hline & Nine-hole Peg Test (9HPT) & $X$ & & $X$ & $x$ & $x$ & $X$ & $x$ \\
\hline & Jebsen Taylor Test (JTT) & $x$ & & $x$ & $x$ & $x$ & $x$ & \\
\hline \multicolumn{9}{|l|}{ Soci(et)al participation } \\
\hline & $\begin{array}{l}\text { Stroke Impact Scale (SIS; } \\
\text { hand function subscale } \\
+ \text { 'thermometer' of well- } \\
\text { being) }\end{array}$ & $x$ & & & $x$ & $x$ & $x$ & \\
\hline & Modified Rankin Scale & $x$ & & & $x$ & $x$ & $x$ & $x$ \\
\hline & $\begin{array}{l}\text { Hospital Anxiety and } \\
\text { Depression Scale (HADS) }\end{array}$ & $x$ & & & $x$ & $x$ & $x$ & \\
\hline
\end{tabular}

The first assessment (TO) takes place in the first 7-14 days post-stroke. The follow-up assessments are at the last day of the stimulation session (T1), and at 1 week (T2), 1 month (T3) after stimulation, 3 months (T4), six months (T5) and 1 year (T6) post-stroke.

rated on 4-point ordinal scales (0 to 3), with a maximum score of 57 (best performance). By removing four items, a hierarchical one-dimensional scale has been constructed. ${ }^{54}$ The ARAT has shown good psychometric properties in patients with stroke with mild to moderate motor severity and without severe cognitive impairment. It has also evidence of unidimensionality, predictive validity and reliability. The ARAT at 3 months will be performed by an independent assessor who will be blinded to treatment allocation.

\section{Secondary outcome measures}

In addition to the ARAT score, we will measure sensorimotor function with the following tests: Fugl-Meyer score test, Nine Hole Peg Test, Jebson-Taylor hand test, and the Stroke Upper Limb Capacity Scale (SULCS) test. The Fugl-Meyer arm score test is a reliable and valid motor performance test consisting of 33 tasks executed with the affected upper limb. ${ }^{55,56}$ Performance on each task is rated as 0,1 or 2 , with higher ratings representing better performance. The Nine Hole Peg Test examines the speed of movement of fine motor skills. The duration of the task execution will be measured. The maximum allowed time will be 50s, during which the number of pegs is counted. ${ }^{57}$ The Nine Hole Peg Test has demonstrated good reliability and validity and has the ability to be used across the 
age span. ${ }^{57}$ Hand skill will be measured with the Jebsen-Taylor hand test. The scores on all seven items, representing activities during daily living, will be summed for a total score. The Jebsen Taylor Test is a reliable and valid measure of gross functional dexterity. ${ }^{58}$ The SULCS assesses arm function capacity, ansd basal and complex hand function capacity. It consists of 10 items, each of which is scored with 0 or 1 . The SULCS has shown good psychometric properties and assesses upper limb capacity according to the International Classification of Functioning, Disability and Health definition. ${ }^{59}$

The following outcome measurements will be used to measure dependency, quality of life and depression: modified Rankin Scale, Stroke Impact Scale and Hospital Anxiety and Depression Scale. The modified Rankin Scale assesses disability and is subdivided into six scores. Score ' 0 ' corresponds to no symptoms whereas score ' 5 ' corresponds to severe handicap. When adhering to a series of rules and a structured interview, the modified Rankin Scale proved to be a reliable and valid measure. ${ }^{60}$ The Stroke Impact Scale is a self-report health status measure, specifically developed for the stroke population. This multidimensional instrument measures hand function, strength, activities of daily living, communication, emotion, memory and thinking. The Stroke Impact Scale has shown good psychometric properties in a diverse group of stroke survivors. ${ }^{61}$ The Hospital Anxiety and Depression Scale measures the core symptoms of anxiety and depression without involving physical symptoms. ${ }^{62}$ This scale is commonly used in stroke patients and has good psychometric properties. ${ }^{63}$

Corticospinal excitability and intracortical inhibition will be assessed from the amplitude and latency of MEP responses, respectively, induced by single-pulse TMS to the ipsi- and contralesional primary motor cortex, measured by EMG with surface electrodes over the FDI muscle of both hands. Stimulus-response curves will be measured from $12 \mathrm{MEP}$ recordings at $95 \%, 105 \%, 115 \%, 125 \%$ and $135 \%$ stimulus intensity relative to the resting motor threshold (in random order).

To assess patients' functional brain status, we will measure functional MRI (fMRI)-based sensorimotor activation (e.g. percentage of blood oxygenation-level dependent change) and resting-state fMRI-based functional connectivity in ipsilesional and contralesional sensory and motor regions. In addition, we will apply diffusion tensor imaging (DTI) to assess structural integrity of the bilateral corticospinal tract and other white matter areas, based on different diffusion parameters (e.g. fractional anisotropy). MRI will be executed on a clinical 3T scanner. Task-related fMRI will be done during flexion-extension movement of the fingers of the hand in a blocked design. Before MRI scanning, patients will be trained to perform the task correctly. The secondary outcome measures including moment of administering can be found in Tables 6.1 and 6.2. 
Table 6.2: Overview of neural outcome measures (including timing)

\begin{tabular}{lllllllll}
\hline & Instrument & T0 & T1 & T2 & T3 & T4 & T5 & T6 \\
\hline $\begin{array}{l}\text { Brain status } \\
\begin{array}{l}\text { Corticospinal excitability and } \\
\text { intracortical inhibition }\end{array}\end{array}$ & Diagnostic TMS & X & X & X & X & X & X & X \\
$\begin{array}{l}\text { Ischemic injury, white matter } \\
\text { integrity, functional connectivity, } \\
\text { and cortical activation }\end{array}$ & (f)MRI (optional) & X & & X & & X & X & X \\
\hline
\end{tabular}

The first assessment (TO) takes place in the first 7-14 days post-stroke. The follow-up assessments are at the last day of the stimulation session (T1), and at 1 week (T2), 1 month (T3) after stimulation, 3 months (T4), six months (T5) and 1 year (T6) post-stroke.

\section{Other baseline data and parameters}

The following parameters will be screened from the medical records of patients and from questionnaires to control for possible confounding effects (see Table 6.3 for an overview and the timing):

1. Use of alcohol or drugs in previous 12 hours, use of caffeine in previous 2 hours and quality of last night's sleep prior to brain stimulation;

2. Complaints of dizziness, headache, insult, tiredness, muscle stiffness and so on after brain stimulation;

3. Medication (that lowers the seizure threshold);

4. Amount of (physical) therapy and self-practice;

5. Demographic parameters at baseline: gender, age, education level, handedness, marital status, ethnicity;

6. Stroke-related parameters at baseline: type of stroke, stroke severity (National Institutes of Health Stroke Scale), side affected limb, days since stroke onset, cognition (Montreal Cognitive Assessment).

\section{Data management}

Data will be collected in an electronic Case Report Form (CRF). Data will be stored on a password-protected electronic server (OpenClinica V.2.0). This is only accessible by the researchers, according to the authorization form. Data will be analyzed on completion of the study. Participants will be given an anonymous study ID to protect confidentiality, and only investigators will have access to the final trial data set. 
Table 6.3: Overview of measures that are part of care as usual and extra care

\begin{tabular}{|c|c|c|c|c|c|c|c|c|c|}
\hline & Instrument & TO & Treatment & T1 & T2 & T3 & T4 & T5 & T6 \\
\hline \multicolumn{10}{|l|}{$\begin{array}{l}\text { Activities of } \\
\text { daily living }\end{array}$} \\
\hline & Barthel Index & $x$ & & & $X^{\star}$ & $X^{\star}$ & $x^{*}$ & $X^{\star}$ & $X^{*}$ \\
\hline \multicolumn{10}{|c|}{$\begin{array}{l}\text { Demographics } \\
\text { and stroke } \\
\text { characteristics }\end{array}$} \\
\hline & $\begin{array}{l}\text { Age, gender, } \\
\text { education, marital } \\
\text { status, ethnicity, work } \\
\text { status, handedness }\end{array}$ & $x$ & & & & & & & \\
\hline & $\begin{array}{l}\text { Montreal Cognitive } \\
\text { Assessment (MOCA) }\end{array}$ & $x$ & & & & & & & \\
\hline & $\begin{array}{l}\text { Type of stroke, stroke } \\
\text { severity (NIHSS), side } \\
\text { affected limb }\end{array}$ & $x$ & & & & & & & \\
\hline $\begin{array}{l}\text { Other } \\
\text { parameters }\end{array}$ & $\begin{array}{l}\text { Use of alcohol/ } \\
\text { caffeine/drugs, } \\
\text { medication, (physical) } \\
\text { therapy/self-practice, } \\
\text { post-stimulation } \\
\text { complaints }\end{array}$ & & $X^{*}$ & $X^{*}$ & $x^{\star}$ & & & & \\
\hline
\end{tabular}

The first assessment (TO) takes place in the first 7-14 days post-stroke. The follow-up assessments are at the last day of the stimulation session (T1), and at 1 week (T2), 1 month (T3) after stimulation, 3 months (T4), six months (T5) and 1 year (T6) post-stroke. * Extra care.

\section{Statistics}

\section{Sample size}

Total sample size will be 60 patients, 30 patients per group, based on a recent meta-analysis that showed a mean effect size on motor outcome after rTMS of 0.55 with a $95 \%$ confidence interval of 0.18 (statistical programme G* power, statistical power $80 \%$ ). 64,65

\section{Statistical analyses}

ARAT scores will be statistically analyzed using repeated measures analysis of variance (ANOVA) with 'time' (different time-points before and after treatment) as within-subject factor and 'treatment' (real cTBS vs. sham cTBS) as between-subject factor. Paired t-tests with correction for multiple comparisons will be used for post hoc analysis. Before entering the data in ANOVA, we will check for normal distribution with the Kolmogorov-Smirnov test. Alternatively, Wilcoxon signed-rank tests will be used to analyze ARAT scores. 
Secondary outcome parameters, i.e. the additional sensorimotor function measures, disability/quality of life scores, and measures of corticospinal excitability and intracortical inhibition, will be analyzed in the same way as described for ARAT scores.

Statistical analysis of MRI parameters will involve repeated measures ANOVA with 'time' (different time-points before and after treatment) as within-subject factor and 'treatment' (real cTBS vs. sham cTBS) as between-subject factor, followed by post hoc t-testing with correction for multiple comparisons. For predictive modeling we will employ generalized linear model-based (GLM) algorithms, ${ }^{66}$ but we may also use alternative algorithms that we have recently tested on their ability to predict infarction based on multiparametric MRI. ${ }^{67}$

All patients will be included in the analyses following an intention-to-treat approach. We do not plan to perform any interim analyses.

\section{Ethics and dissemination}

\section{Ethics}

The study has been approved by the Medical Research Ethics Committee of the University Medical Center Utrecht.

Before inclusion, patients should have read the patient information letter, which they may discuss with their relatives, to understand the goal and execution plan of the trial. After written informed consent patients can participate. Before the first examination, a researcher will restate the study information, and patients will (again) be informed about the possibility to ask questions about the study. Furthermore, the option to withdraw from the study at any time will be explained. If the patient is not able to write down the needed information (because of hand/arm disability), a relative can fill out the informed consent form. The study will be conducted in accordance with the principles of Good Clinical Practice, the Medical Research Involving Human Subjects act (WMO) and the Declaration of Helsinki. All protocol changes such as modifications in eligibility criteria, outcome measures, analyses or study procedures will be submitted to the Medical Research Ethics Committee.

\section{Safety}

Adverse events are defined as any undesirable medical experience occurring to a subject during the study, whether or not considered related to cTBS. All adverse events reported spontaneously by the subject to (or observed by) the investigator or his/her staff will be recorded for the period of the treatment (two weeks) and for an additional week after the treatment has ended. Seizures, which are the most serious rTMS-related side effect with a crude risk of approximately $0.02 \%,{ }^{50}$ would only be expected to occur during or immediately 
after rTMS trains. Furthermore, all adverse events occurring within 24 hours after MRI will be reported. Any serious event will be immediately reported to the Medical Research Ethics Committee of the University Medical Center Utrecht.

An internal Data Safety and Monitoring Board (DSMB) at the University Medical Center Utrecht has been established to perform ongoing safety surveillance. A temporary, independent project-specific member is added to the internal DSMB. The internal DSMB will monitor the progress (randomization, losses to follow-up) and safety (evidence for significant treatment harm) aspects of the study. The internal DSMB may also advise on protocol modifications suggested by investigators or sponsors and assess impact and relevance of external evidence.

\section{Dissemination}

The results will be disseminated through (open access) peer-reviewed publications, networks of scientists, patient associations (like 'Hersenletsel.nl' and 'Kennisnetwerk CVA NL'), professionals and the public, and presented at relevant conferences. Participants of the study will be updated about the progress and results of the study by newsletters. Patient engagement will be achieved by involving patients in the development of the protocol and script, for example, in improving and refining the motor task during fMRI.

\section{Acknowledgments}

We thank Wilma Jentink and Annet Slabbekoorn for their input on the protocol.

\section{Funding}

This work was supported by the Netherlands Organization for Scientific Research (VICl 016.130.662).

\section{References}

1. Nichols-Larsen, D. S., Clark, P. C., Zeringue, A., Greenspan, A. \& Blanton, S. Factors influencing stroke survivors' quality of life during subacute recovery. Stroke 36, 1480-1484 (2005).

2. Broeks, J. G., Lankhorst, G. J., Rumping, K. \& Prevo, A. J. The long-term outcome of arm function after stroke: results of a follow-up study. Disabil. Rehabil. 21, 357-364 (1999).

3. Feigin, V. L., Lawes, C. M. M., Bennett, D. A. \& Anderson, C. S. Stroke epidemiology: A review of population-based studies of incidence, prevalence, and case-fatality in the late 20th century. Lancet Neurol. 2, 43-53 (2003). 
4. Page, S. J., Gater, D. R. \& Bach-Y-Rita, P. Reconsidering the motor recovery plateau in stroke rehabilitation. Arch. Phys. Med. Rehabil. 85, 1377-1381 (2004).

5. Hosomi, K. et al. Daily repetitive transcranial magnetic stimulation for poststroke upper limb paresis in the subacute period. J. Stroke Cerebrovasc. Dis. 25, 1655-1664 (2016).

6. Sunderland, A. et al. Enhanced physical therapy for arm function after stroke: a one year follow up study. J. Neurol. Neurosurg. Psychiatry 57, 856-858 (1994).

7. Kwakkel, G., Kollen, B. J., Van der Grond, J. V. \& Prevo, A. J. H. Probability of regaining dexterity in the flaccid upper limb: Impact of severity of paresis and time since onset in acute stroke. Stroke 34, 2181-2186 (2003).

8. Wade, D. \& Langton-Hewer, R. The hemiplegic arm after stroke: measurement and recovery. J. Neurol. Neurosurgery, Psychiatry 46, 521-524 (1983).

9. Luke, C., Dodd, K. J. \& Brock, K. Outcomes of the Bobath concept on upper limb recovery following stroke. Clin. Rehabil. 18, 888-898 (2004).

10. Kang, N., Summers, J. J. \& Cauraugh, J. H. Non-Invasive Brain Stimulation Improves Paretic Limb Force Production: A Systematic Review and Meta-Analysis. Brain Stimul. 9, 662-670 (2016).

11. Duncan, P. W., Goldstein, L. B., Matchar, D., Divine, G. W. \& Feussner, J. Measurement of motor recovery after stroke. Stroke 23, 1084-1089 (1992).

12. Bolognini, N., Pascual-Leone, A. \& Fregni, F. Using non-invasive brain stimulation to augment motor training-induced plasticity. J. Neuroeng. Rehabil. 6, 8 (2009).

13. Nakayama, H., Jørgensen, H. S., Raaschou, H. O. \& Olsen, T. S. Recovery of upper extremity function in stroke patients: the Copenhagen Stroke Study. Arch. Phys. Med. Rehabil. 75, 394-398 (1994).

14. Buma, F., Kwakkel, G. \& Ramsey, N. Understanding upper limb recovery after stroke. Restor. Neurol. Neurosci. 31, 707-722 (2013).

15. Hoyer, E. H. \& Celnik, P. A. Understanding and enhancing motor recovery after stroke using transcranial magnetic stimulation. Restor. Neurol. Neurosci. 29, 395-409 (2011).

16. Le, Q., Qu, Y., Tao, Y. \& Zhu, S. Effects of repetitive transcranial magnetic stimulation on hand function recovery and excitability of the motor cortex after stroke: A meta-analysis. Am. J. Phys. Med. Rehabil. 93, 1-9 (2014).

17. Liew, S., Santarnecchi, E., Buch, E. \& Cohen, L. Non-invasive brain stimulation in neurorehabilitation: local and distant effects for motor recovery. Front. Hum. Neurosci. 8, 1-15 (2014).

18. Simonetta-Moreau, M. Non-invasive brain stimulation (NIBS) and motor recovery after stroke. Ann. Phys. Rehabil. Med. 57, 530-542 (2014).

19. Wessel, M. J., Zimerman, M. \& Hummel, F. C. Non-invasive brain stimulation: an interventional tool for enhancing behavioral training after stroke. Front. Hum. Neurosci. 9, 265 (2015).

20. Lefaucheur, J. P. et al. Evidence-based guidelines on the therapeutic use of repetitive transcranial magnetic stimulation (rTMS). Clin. Neurophysiol. 125, 2150-2206 (2014).

21. Hummel, F. C. \& Cohen, L. G. Non-invasive brain stimulation: a new strategy to improve neurorehabilitation after stroke? Lancet Neurol. 5, 708-712 (2006).

22. Hallett, M. Transcranial Magnetic Stimulation: A Primer. Neuron 55, 187-199 (2007).

23. Di Pino, G. et al. Modulation of brain plasticity in stroke: a novel model for neurorehabilitation. Nat. Rev. Neurol. 10, 597-608 (2014).

24. Muller, P. A. et al. Suppression of motor cortical excitability in anesthetized rats by low frequency repetitive transcranial magnetic stimulation. PLoS One 9, 1-8 (2014).

25. Huang, Y. Z., Edwards, M. J., Rounis, E., Bhatia, K. P. \& Rothwell, J. C. Theta burst stimulation of the human motor cortex. Neuron 45, 201-206 (2005).

26. Rossini, P. M. et al. Non-invasive electrical and magnetic stimulation of the brain, spinal cord, roots and peripheral nerves: Basic principles and procedures for routine clinical and research application: An updated report from an I.F.C.N. Committee. Clin. Neurophysiol. 126, 1071-1107 (2015). 
27. Ackerley, S. J., Stinear, C. M., Barber, P. A. \& Byblow, W. D. Priming sensorimotor cortex to enhance task-specific training after subcortical stroke. Clin. Neurophysiol. 125, 1451-1458 (2014).

28. Talelli, P. et al. Theta burst stimulation in the rehabilitation of the upper limb: a semirandomized, placebo-controlled trial in chronic stroke patients. Neurorehabil. Neural Repair 26, 976-987 (2012).

29. Meehan, S. K., Dao, E., Linsdell, M. A. \& Boyd, L. A. Continuous theta burst stimulation over the contralesional sensory and motor cortex enhances motor learning post-stroke. Neurosci. Lett. 500, 26-30 (2011).

30. Di Lazzaro, V. et al. Inhibitory theta burst stimulation of affected hemisphere in chronic stroke: A proof of principle, sham-controlled study. Neurosci. Lett. 553, 148-152 (2013).

31. Delvaux, V. et al. Post-stroke reorganization of hand motor area: A 1-year prospective follow-up with focal transcranial magnetic stimulation. Clin. Neurophysiol. 114, 1217-1225 (2003).

32. Ward, N. S., Brown, M. M., Thompson, A. J. \& Frackowiak, R. S. J. Neural correlates of motor recovery after stroke: A longitudinal fMRI study. Brain 126, 2476-2496 (2003).

33. Murase, N., Duqye, J., Mazzocchio, R. \& Cohen, L. G. Influence of interhemispheric interactions on motor function in chronic stroke. Ann. Neurol. 55, 400-409 (2004).

34. Duque, J. et al. Transcallosal inhibition in chronic subcortical stroke. Neuroimage 28, 940-946 (2005).

35. Bloom, J. S. \& Hynd, G. W. The role of the corpus callosum in interhemispheric transfer of information: Excitation or inhibition? Neuropsychol. Rev. 15, 59-71 (2005).

36. Nowak, D. A., Grefkes, C., Ameli, M. \& Fink, G. R. Interhemispheric competition after stroke: brain stimulation to enhance recovery of function of the affected hand. Neurorehabil. Neural Repair 23, 641-656 (2009).

37. Grefkes, C. \& Fink, G. R. Noninvasive brain stimulation after stroke. Curr. Opin. Neurol. 29, 1 (2016).

38. Grefkes, C. et al. Cortical connectivity after subcortical stroke assessed with functional magnetic resonance imaging. Ann. Neurol. 63, 236-246 (2008).

39. Chang, W. H. et al. Long-term effects of rTMS on motor recovery in patients after subacute stroke. J. Rehabil. Med. 42, 758-764 (2010).

40. Khedr, E. M., Etraby, A. E., Hemeda, M., Nasef, A. M. \& Razek, A. A. E. Long-term effect of repetitive transcranial magnetic stimulation on motor function recovery after acute ischemic stroke. Acta Neurol. Scand. 121, 30-37 (2010).

41. Huynh, W., Vucic, S., Krishnan, A. V, Lin, C. S.-Y. \& Kiernan, M. C. Exploring the Evolution of Cortical Excitability Following Acute Stroke. Neurorehabil. Neural Repair 30, 244-257 (2016).

42. Amengual, J. L. et al. Prognostic value of cortically induced motor evoked activity by TMS in chronic stroke: Caveats from a revealing single clinical case. BMC Neurol. 12, 35 (2012).

43. Ackerley, S. J., Stinear, C. M., Barber, P. A. \& Byblow, W. D. Combining theta burst stimulation with training after subcortical stroke. Stroke 41, 1568-1572 (2010).

44. Ackerley, S. J. et al. Primed physical therapy enhances recovery of upper limb function in chronic stroke patients. Neurorehabil. Neural Repair 30, 339-348 (2016).

45. Khedr, E. M., Ahmed, M. A., Fathy, N. \& Rothwell, J. C. Therapeutic trial of repetitive transcranial magnetic stimulation after acute ischemic stroke. Neurology 65, 466-468 (2005).

46. Khedr, E. M., Abdel-Fadeil, M. R., Farghali, A. \& Qaid, M. Role of 1 and $3 \mathrm{~Hz}$ repetitive transcranial magnetic stimulation on motor function recovery after acute ischaemic stroke. Eur. J. Neurol. 16, 1323-1330 (2009).

47. Sasaki, N., Mizutani, S., Kakuda, W. \& Abo, M. Comparison of the effects of high- and low-frequency repetitive transcranial magnetic stimulation on upper limb hemiparesis in the early phase of stroke. J. Stroke Cerebrovasc. Dis. 22, 413-418 (2013). 
48. Sasaki, N., Kakuda, W. \& Abo, M. Bilateral high-and low-frequency rTMS in acute stroke patients with hemiparesis: A comparative study with unilateral high-frequency rTMS. Brain Inj. 28, 1362-1301 (2014).

49. Stinear, C. M., Barber, P. A., Petoe, M., Anwar, S. \& Byblow, W. D. The PREP algorithm predicts potential for upper limb recovery after stroke. Brain 135, 2527-2535 (2012).

50. Rossi, S. et al. Safety, ethical considerations, and application guidelines for the use of transcranial magnetic stimulation in clinical practice and research. Clin. Neurophysiol. 120, 2008-2039 (2009).

51. Goldsworthy, M. R., Pitcher, J. B. \& Ridding, M. C. A comparison of two different continuous theta burst stimulation paradigms applied to the human primary motor cortex. Clin. Neurophysiol. 123, 2256-2263 (2012).

52. Lee, J. Y. et al. Is MRI a reliable tool to locate the electrode after deep brain stimulation surgery? Comparison study of CT and MRI for the localization of electrodes after DBS. Acta Neurochir. (Wien) 152, 2029-2036 (2010).

53. Sauner, D. et al. Multimodal localization of electrodes in deep brain stimulation: Comparison of stereotactic ct and mri with teleradiography. Stereotact. Funct. Neurosurg. 88, 253-258 (2010).

54. van der Lee, J. H., Roorda, L. D., Beckerman, H., Lankhorst, G. J. \& Bouter, L. M. Improving the Action Research Arm test: a unidimensional hierarchical scale. Clin. Rehabil. 16, 646-653 (2002).

55. Badke, M. B. \& Duncan, P. W. Patterns of rapid motor responses during postural adjustments when standing in healthy subjects and hemiplegic patients. Phys. Ther. 63, 13-20 (1983).

56. Duncan, P. W., Propst, M. \& Nelson, S. G. Reliability of the Fugl-Meyer assessment of sensorimotor recovery following cerebrovascular accident. Phys. Ther. 63, 1606-1610 (1983).

57. Grice, K. O. et al. Adult norms for a commercially available nine hole peg test for finger dexterity. Am. J. Occup. Ther. 57, 570-573 (2003).

58. Wang, Y. C. et al. Assessing dexterity function: A comparison of two alternatives for the NIH toolbox. J. Hand Ther. 24, 313-321 (2011).

59. Bovend'Eerdt, T. J. H., Dawes, H., Johansen-Berg, H. \& Wade, D. T. Evaluation of the Modified Jebsen Test of Hand Function and the University of Maryland Arm Questionnaire for Stroke. Clin. Rehabil. 18, 195-202 (2004).

60. Houwink, A., Roorda, L. D., Smits, W., Molenaar, I. W. \& Geurts, A. C. Measuring upper limb capacity in patients after stroke: Reliability and validity of the stroke upper limb capacity scale. Arch. Phys. Med. Rehabil. 92, 1418-1422 (2011).

61. Kasner, S. E. Clinical interpretation and use of stroke scales. Lancet Neurol. 5, 603-612 (2006).

62. Duncan, P. W., Bode, R. K., Lai, S. M. \& Perera, S. Rasch analysis of a new stroke-specific outcome scale: The stroke impact scale. Arch. Phys. Med. Rehabil. 84, 950-963 (2003).

63. Snaith, R. P. \& Zigmond, A. S. The hospital anxiety and depression scale. Acta Psychiatr. Scand. 67, 361-370 (1983).

64. Faul, F., Erdfelder, E., Lang, A.-G. \& Buchner, A. G*Power 3: a flexible statistical power analysis program for the social, behavioral, and biomedical sciences. Behav. Res. Methods 39, 175-191 (2007).

65. Hsu, W. Y., Cheng, C. H., Liao, K. K., Lee, I. H. \& Lin, Y. Y. Effects of repetitive transcranial magnetic stimulation on motor functions in patients with stroke: A meta-analysis. Stroke 43, 1849-1857 (2012).

66. Wu, O., Dijkhuizen, R. M. \& Sorensen, A. G. Multiparametric Magnetic Resonance Imaging of Brain Disorders. Top. Magn. Reson. Imaging 21, 129 (2010).

67. Bouts, M. J. R. J. et al. Early identification of potentially salvageable tissue with MRI-based predictive algorithms after experimental ischemic stroke. J. Cereb. Blood Flow Metab. 33, 1075-1082 (2013). 


\section{Appendix 6.1 Amendments to B-STARS protocol}

\section{Inclusion criteria}

1) Adult patient age $\geq 18$;

2) first-ever unilateral ischemic and hemorrhagic stroke (i.e. within cerebral hemispheres, brainstem);

3) paresis of one arm, with a SA score shoulder abduction $\geq 9$ (Motricity Index)

4) within the first 3 weeks after stroke onset;

5) signed informed consent.

\section{Exclusion criteria}

Addition of the following remark: N.B. metal fillings (i.e. conductive) or non-ferromagnetic dental implants are an exception to the rule.

\section{Addition of skilled reaching task}

The skilled reaching task assesses skilled reaching behavior and can be scored on multiple components (success score, first attempt, movement elements). Patients will be seated in an armless chair, feet flat on the ground and their hands palm down on their thighs with the fingers extended. A small food item (Honey Loops, smarties, raisin, shelled peanut) will be placed on a pedestal placed in front of them, adjusted to the trunks' height and arm length. Each hand will be used to make three to five reaches, accomplished within a few minutes. The patients will have to reach for the food item and withdraw this to their mouth. The tasks will be video-recorded with a camera with a shutter speed of 1/1000 frames per second, to enable frame-by-frame analysis.

\section{Single-pulse TMS substituted by grid measurements}

Motor-evoked potentials (MEPs) evoked by single pulse TMS to the motor hotspot in the ipsi- and contralesional hemisphere will be assessed at TO-T6. Single pulse TMS will be delivered to several targets in a grid which is virtually projected onto the left and right primary motor cortices to visualize the changes in cortical motor representation. During the grid registration, EMG will be measured in the bilateral first dorsal interosseous, contralesional anterior pollicis brevis and contralesional abductor digiti minimi muscles. The grid is centered at the maximum MEP amplitude (as determined during the resting motor threshold procedure) and consists of 4 by 4 targets with $8 \mathrm{~mm}$ spacing between the targets. 5 repetitions of single pulse TMS will be delivered at $120 \%$ relative to the resting motor threshold to each target. In total, 125 pulses will be delivered. A beta version of the neural navigator software will be used to register the TMS coil position at the moment of TMS pulse delivery and visualization of this location on the brain surface. 


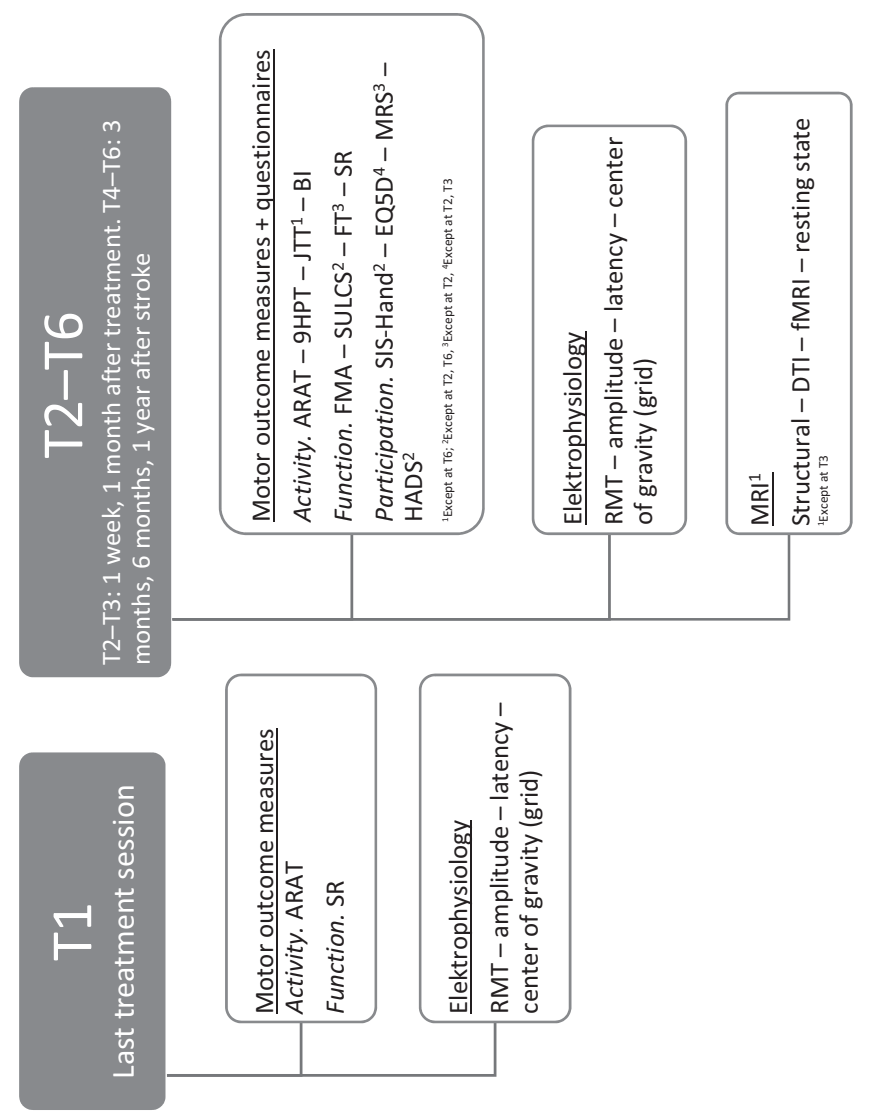

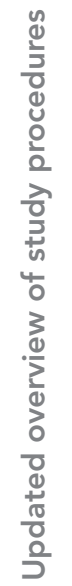

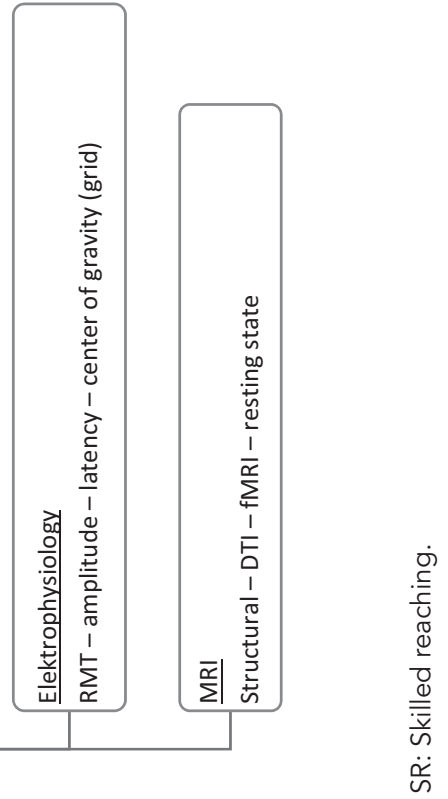




\section{Exploring the experiences of stroke patients treated with transcranial magnetic stimulation for upper limb recovery: A qualitative study}

Eline C.C. van Lieshout

Lilliane D. Jacobs Maaike Pelsma

Rick M. Dijkhuizen

Johanna M.A. Visser-Meily 


\section{Abstract}

Background: Transcranial magnetic stimulation (TMS) treatments have shown promise in improving arm recovery in stroke patients. Currently, little is known about patients' experiences with TMS treatment, and this lack of knowledge may affect optimal implementation in clinical practice. The aim of this explorative study was to gain insight in the perceived effects and experiences of the design and delivery of a TMS treatment for upper limb recovery from the perspectives of stroke patients.

Methods: This qualitative study was conducted as part of a randomized controlled trial $(\mathrm{RCT})$ in a specialized rehabilitation center. Data were collected through face-to-face semistructured interviews with 13 stroke patients who completed a 10-day TMS intervention for upper limb recovery. The interviews were recorded, transcribed verbatim and analyzed using thematic analysis.

Results: The major themes that emerged from the patients' feedback were the following: positive experiences of the treatment (experienced physical effects, comfort, therapeutic relationship, receiving information, learning about the brain, no burden of added rTMS treatment session, no unpleasant aspects), concerns (effects of stimulation of the brain, equipment, logistics), general experience of recovery, experienced psychological effects (grateful, sense of purpose, recovery as extra motivation to exercise, disappointment and hope of group allocation), and motivation to participate (personal benefit and cognitions, altruism). Important components related to the positive experience of the design and delivery of the treatment included comfort (i.e. moment of relaxation) and the sensation of a painless treatment without side-effects. Key concerns included uncertainty and anxiety about possible negative consequences and group allocation.

Conclusions: This study demonstrates that TMS is well accepted by stroke patients with an upper limb paresis. Besides the expectation of a therapeutic benefit, the patients reported various psychological effects. Positive experiences, such as the provision of a short moment of relaxation each day, could have practical implications for clinical stroke rehabilitation settings aimed at improving patient satisfaction. Explanation about and feedback from routine motor recovery progression monitoring at fixed times post-stroke is also valued by patients. Negative emotions may be limited or avoided by transparent and recurrent information delivery in future trials. 


\section{Background}

Stroke is one of the leading causes of disability and can have severe consequences for upper limb function. ${ }^{1}$ Patients with impaired upper limb function often experience limitations in activities, and restrictions in participation, with a consequent decline in health-related quality of life..$^{2,3}$ While less than $50 \%$ of stroke patients without initial hand capacity on admission in a rehabilitation center regains some hand capacity at discharge, more than $75 \%$ of patients with residual hand capacity regain advanced hand capacity at discharge. ${ }^{4}$ Thus, many patients experience at least some degree of recovery of their lost motor function over time. ${ }^{5}$ Restorative therapies that lead to a full return of all behaviors as before injury are currently under study. ${ }^{6,7}$ Systematic reviews and meta-analyses of studies performed in the acute to chronic phases post-stroke have shown that non-invasive brain stimulation (NIBS) can induce therapeutic effects on upper limb function. ${ }^{6-8}$ Very recently, level A evidence (definite efficacy) has been indicated for low-frequency rTMS of the contralesional primary motor cortex (M1) in the post-acute stage (one week to two months post-stroke) for improvement of upper limb motor function in stroke patients. ${ }^{9}$

NIBS techniques, such as repetitive transcranial magnetic stimulation (rTMS) and transcranial direct current stimulation (tDCS), have the potential to increase or decrease cortical excitability, dependent on the parameters of stimulation. ${ }^{10-12}$ In a multi-institutional study, only transient side effects, namely minor dizziness, discomfort at stimulation site, and mild headache, were reported after rTMS by 22 out of 1,725 patients. ${ }^{13}$ This confirms the good tolerance for this type of treatment. An interview study that explored the views and experiences of 21 patients who underwent tDCS combined with robotic therapy for upper limp recovery, revealed that the therapy was generally experienced as effective and comfortable. ${ }^{14}$ However, some patients also reported discomfort (e.g. painful, itchy stimulation) or feelings of uncertainty about the consequences of tDCS. So far, the subjective experiences of patients receiving rTMS have not been assessed yet. Insights from the patients' perspectives may help researchers and health care professionals to identify topics that are important for patients undergoing a treatment, which could improve future trial design and subsequent clinical implementation. In addition, patients' expectations can provide important predictors of treatment outcomes. ${ }^{15-17}$

We conducted a qualitative study that ran parallel to a randomized controlled trial (B-STARS), in which stroke patients undergo cTBS (continuous theta burst stimulation), a variant of rTMS, within the first month post-stroke to promote upper limb recovery..$^{18}$ cTBS can decrease cortical excitability and consists of an uninterrupted train of 20 to 40 seconds of TBS. ${ }^{19}$ The treatment starts within 21 days after stroke onset, because the brain may be most responsive to neurorehabilitation in this time-window, and is always followed by upper limb training, 
which is part of inpatient rehabilitation care. The design and methods of the RCT have been described in detail in a protocol article. ${ }^{18}$ The objectives of the current qualitative study were to identify and understand the patients' perceived effects and experiences of the design and delivery of a TMS-based treatment for upper limb recovery. Results from this study could reveal aspects that interfere with or promote effective clinical implementation of rTMS treatment and lead to improvement of future rTMS trials.

\section{Methods}

\section{Design}

Semi-structured interviews were conducted with a subset of patients who participated in the B-STARS (Brain-Stimulation for Arm Recovery after Stroke) trial. ${ }^{18}$ The B-STARS trial is a stratified randomised controlled trial to investigate the effects of rTMS (cTBS) on arm recovery after stroke. The B-STARS trial is still ongoing, and expected to be completed by 2020/2021. The B-STARS is registered with the study number NL5952, November 28, 2016.

\section{B-STARS intervention}

The included patients were randomly allocated to two groups: 1) real or 2) sham cTBS of the contralesional primary motor cortex. Patients underwent 10 treatment sessions in the morning or afternoon, followed by usual care upper limb training, over a period of two weeks during their inpatient rehabilitation period. A treatment session had an average duration of ten minutes. Upper limb therapy started 5 minutes after cTBS and consisted of 60 minutes of upper limb exercises individualized to each patient, delivered by experienced physical therapists. A Neuro-MS/D Advanced Therapeutic stimulator (Neurosoft, Russia) in combination with a $70 \mathrm{~mm}$ figure-of-eight coil was used for cTBS. Sham stimulation was administered with the protocol in sham mode (generating pulses at $90 \%$ lower intensity of the RMT). All patients were naïve to rTMS and therefore should not be able to distinguish between real and sham rTMS. Full details of the B-STARS trial protocol have been described elsewhere. ${ }^{18}$ The study protocol was approved by the Medical Ethics Review Committee of the University Medical Center Utrecht and the participating rehabilitation center. The study was registered in the Dutch Trial Register (NL5952). This study conforms to the consolidated criteria for reporting qualitative research (COREQ) guidelines (Appendix 7.1).

\section{Participants}

Patients met the in- and exclusion criteria of the B-STARS trial ${ }^{18}$ : a first-ever ischemic or hemorrhagic stroke, $\geq 18$ years, $\leq 21$ days post-stroke, a paresis of one arm (Motricity Index 
score $\geq 9$ on shoulder abduction) and the ability to provide informed consent. Patients were excluded if they had a disabling medical history (severe head trauma, severe or recent heart disease, coercively treated at a psychiatric ward), history of epilepsy, normal to almost normal use of the hand (Motricity Index score of 33 on pinch grip ${ }^{20}$ ), severe deficits in communication, memory, or understanding that impede proper study participation, or contra-indications for TMS and MRI. Participants of the B-STARS trial who completed the intervention period were asked to participate by telephone or face-to-face in the qualitative study by EvL or LJ. Participants who were willing to be interviewed were approached for an appointment. Written informed consent had already been obtained for the randomized controlled trial, in which patients could indicate whether they could be approached for the qualitative study after completion of the intervention period.

\section{Data collection}

Patients who took part and completed the rTMS intervention period were asked to be interviewed. To achieve heterogeneity in the sample, we invited patients who completed the trial, as well as consecutively patients who were still in the trial for follow-up measurements. Patients from the real as well as the sham cTBS group were included in order to get a complete picture of the experiences of patients undergoing the treatment. Identification of group differences was not the aim of this paper. The semi-structured interviews were undertaken on a single occasion between May and October 2019. Participants were given the choice of location of the interview: at the rehabilitation center or at the patient's home. The semi-structured interviews were conducted by a female psychologist (LJ) who was not involved in the recruitment and data collection process and who was blinded for the patient's group allocation. Patients were informed about the professional background of the interviewer and the purpose of the interviews. The interviewer was trained in interview techniques before the start of the study. The duration of each interview was between 30 and 60 minutes. The interviews were conducted in Dutch. All interviews were audio-recorded, and field notes about the patient's behaviour and contextual matters were taken during the interview. The interviewer used a topic guide focused on (the motivation for) participation in the treatment; emotions, cognitions and sensations before, during and after the treatment; effect of the treatment on arm function; and expectations in relation to rTMS (for a complete topic guide, see Appendix 7.2). The interviewer briefly explained the purpose of the interview, and techniques as hemming, summarizing and reflecting were used.

\section{Data analysis}

All the audio recordings were transcribed verbatim. The transcripts were crosschecked by the first author (EvL). Confidential information, including health care services, were deleted 
from the transcripts. Qualitative data analysis was conducted by the first (EvL) and second author (LJ). The first author, female psychologist and researcher, knew the patients from the $\mathrm{RCT}$, therefore, involving an external researcher (LJ) to the study minimized risk of bias. The process of data collection and analysis was iterative. Thematic analysis was used to interpret the data, which involved reading and rereading the responses from all interviewed patients and generating initial codes, independently by the two researchers ( $E v L$ and LJ). Thematic analysis was performed according to the six phases as described by Braun and Clarke. ${ }^{21}$ To confirm correct application of the six phases of Braun and Clarke, the 15-point checklist was used (Appendix 7.3). Recurrent themes were identified and listed. The researchers reviewed, compared and discussed the (emerging) themes until the final themes could be determined. In addition, throughout the analysing process, data saturation was discussed. Data saturation was accomplished when no new themes were added during the last three interviews. ${ }^{22}$ Three patients provided feedback on the findings. Appropriate quotes and citations were selected to illustrate each theme. MAXQDA 2018 (VERBI Software, 2017) was used for data analysis.

\section{Results}

Thirteen patients with a mean age of 56.5 (SD 13.0; range 32-77 years) were interviewed. We excluded one patient because his memory deficits gave serious problems during the interview. All but three patients who were approached for an interview agreed to participate. Of these three patients, two could not participate due to logistic reasons and one felt uncomfortable with an interview because of aphasia. The majority of the patients were women (61.5\%). The time post-stroke varied between 1 and 25 months. 61.5\% had a right-sided hemiparesis. Characteristics of the patients are listed in Table 7.1.

The results are presented in two main sections. The first section describes the patient's experiences with the treatment and presents the themes positive experiences with the treatment and concerns. During the interviews the patients also shared experiences about participation in an RCT, which are outlined in the themes experienced psychological effects and motivation to participate. An overview of the themes and subthemes is given in Table 7.2. Patient's quotations are shown in italics, and interpretation of the patient's words is presented alongside the quotations. 


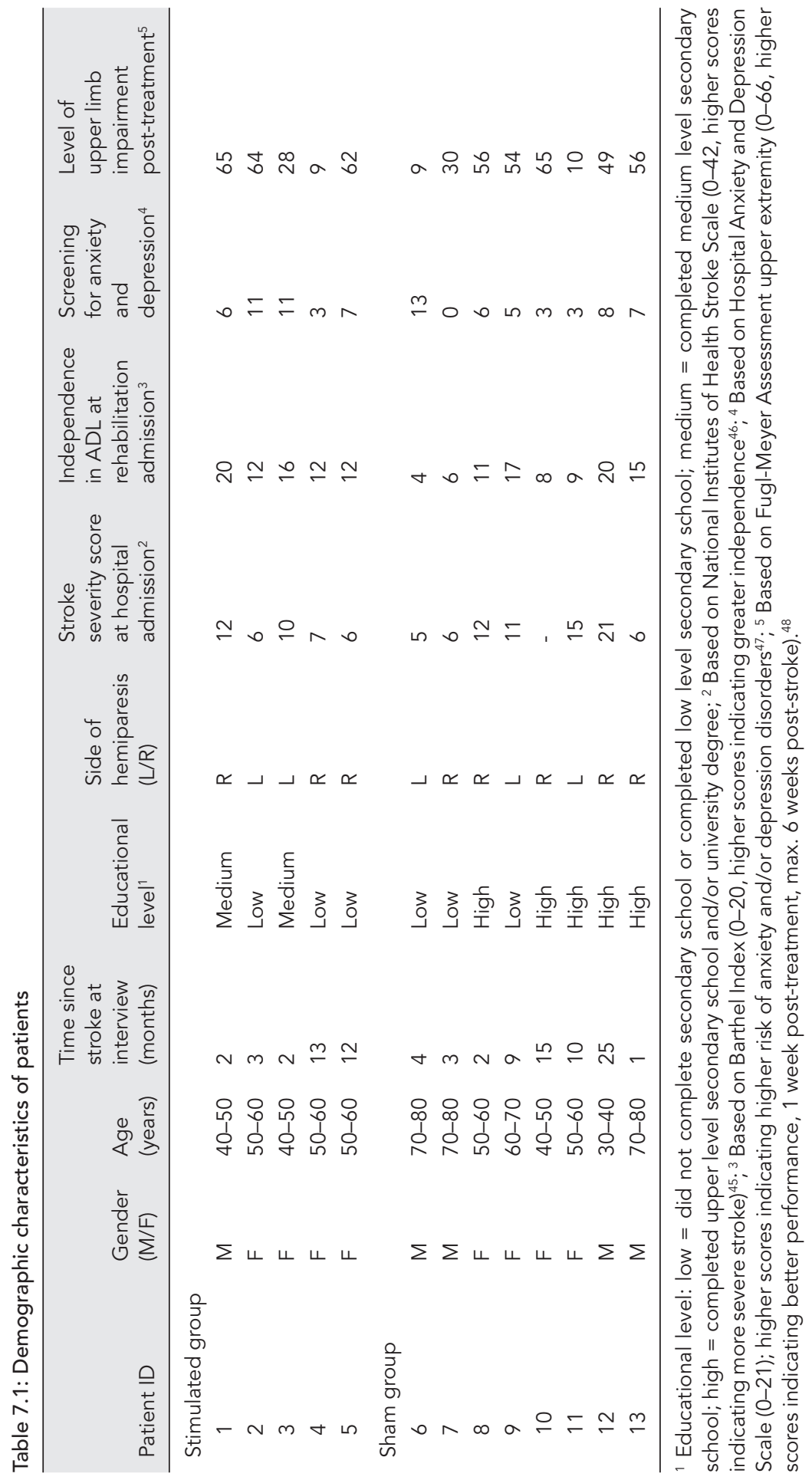


Table 7.2: Overview themes and subthemes

\begin{tabular}{ll}
\hline Patient experiences with the treatment & Subthemes \\
\hline Positive experiences of the treatment & Experienced physical effects \\
& Comfort \\
& Therapeutic relationship \\
& Receiving information \\
& Learning about the brain \\
& No burden of added TMS treatment session \\
& No unpleasant aspects \\
& Effects of stimulation of the brain \\
& Equipment (chair and coil) \\
Concerns & Logistics \\
& \\
\hline Participation in an RCT & \\
\hline General experience of recovery & Grateful \\
Experienced psychological effects & Sense of purpose \\
& Recovery as extra motivation to exercise \\
& Disappointment and hope of group allocation \\
& Personal benefit and cognitions \\
Motivation to participate & Altruism \\
\hline
\end{tabular}

\section{Patient's experiences with the treatment}

\subsection{Positive experiences of the treatment}

In general, patients had positive experiences receiving the rTMS treatment. Several patients mentioned that undergoing the treatment sessions felt as a special moment during their day: "For me it was... it sounds very silly... a getaway within my rehabilitation." (P5) In addition, a good vibe during the treatment sessions was emphasized by patients.

All patients would recommend participation in the intervention to other stroke patients. One patient expressed disappointment when the treatment sessions came to an end.

"I thought it was a pity that it was already over after ten times." (P10)

\section{1a Experienced physical effects}

Some patients reported that the rTMS treatment had improved their arm function. On the contrary, some other patients reported that they were unable to indicate a cause of their recovery. They thought that a combination of participation in the trial, rehabilitation therapies and their own willingness to recover led to improvement. Two patients noted that they did not know the difference between what to expect and what not to expect in terms of recovery (e.g. spontaneous recovery). 
Some patients were unable to identify any possible improvement. Those patients did not notice a difference in arm function, because of the TMS treatment.

"No, no not immediately. Maybe yes, of course you never know what the effect would be if you didn't do it. I have also noticed that with the medication that I take, since I'm tapering off, it suddenly gets very bad. So [the medication] did not lead to improvement, but if you do not take it, it affects you negatively. Perhaps that it also works that way for this [B-STARS intervention]." (P12)

Some patients express the difficulty of experiencing improvement when there is no comparison.

"I do not dare to say that. I don't know how to explain that. I got this [a stroke].

I don't know what it would be like if I hadn't done it. With or without [therapy],

I don't have any comparison, so maybe it helped but maybe it did not." (P9)

One patient stated that it was probably too early to speak about the results: "I think it's too early to judge." (P13)

A few patients reported no (long-term) improvements in arm function following the treatment.

Often patients reported their thoughts when they had sensations in the hand muscles in response to the pulses given to the head during determination of the resting motor threshold (RMT). Those sensations often gave rise to the idea that the treatment was 'working'. The patients were aware that the determination of the RMT was not part of the treatment, but of the set-up phase.

\section{1b Comfort}

The treatment itself was described as a relaxed experience. Patients felt comfortable and were not nervous or anxious during the treatment sessions. Two patients described their voluntary participation in the trial.

"Yes, because otherwise you could have stopped it [treatment], if it became too much, but it didn't."

The comfort of the chair where they had to sit in was highly appreciated. The chair was experienced as very relaxing and some patients almost fell asleep in it. The calm environment without too many noises, and the calmness that the researchers radiated (e.g. by talking softly) seemed to contribute to the relaxed experience that patients had in the trial. A couple of patients described the pulses given on the head as a relaxing experience. One patient described it as follows: 
"During the treatment I was very calm and strangely enough those pulses were calming and almost made me fall asleep." (P3)

\section{1c Therapeutic relationship}

All patients spoke highly of the research team that delivered the treatment. The researchers were seen as 'polite', 'correct', 'friendly' and 'patient'. The human approach by the research team was experienced as comforting. For example, patients described that the researchers took their time in communicating with them and patients did not feel as a 'number'. One patient was particularly pleased to receive attention that was specifically intended for him: the contact was more intimate.

Some patients expressed that they felt the researchers were grateful that they participated in the trial.

"And I have to say you were always very friendly. Grateful. I always consider that to be important. However, it is not a decisive factor, but it is always nice when you're finished and people are happy with what you have done. If you can help someone, why not." (P12)

The attitude of the researchers was described as professional and trustworthy.

"No, because you appear so confident and reliable. You know what you are talking about, in my opinion, that is very obvious. Not like: 'maybe when we try this or that', then I would sense doubt. But, now it is clear what we are going to do and how we are going to do it." (P7)

Within these narratives there is a strong sense of safety. One patient expressed appreciation for the communicated zero expectations from the researchers.

\section{1d Receiving information}

It was experienced that the research team gave clear information. Different aspects of information delivery were valued. One patient explained: "Just good information, normal answers, calmly - they take the time to explain it." (P2) Another said: "Everything was explained very neatly in regular Dutch language, the way I speak it." (P7) Many patients commented on the explanation of every step during the treatment sessions.

"And they also said beforehand 'we are going to do this now, we are doing that now, you will feel this right now.' I also really like that." (P13)

Knowing what to expect was emphasized as one of the reasons for the positive information exchange. 


\section{1e Learning about the brain}

The muscle sensations of the pulses had a funny component for patients and made them more aware of the working of the brain.

"Well I didn't find it exciting, scary or anything. Yes, really intrinsic interest in how the brain works and what is connected within your body." (P10)

\section{1f No burden of added TMS treatment session}

All patients perceived participation in the daily treatment sessions as an element of their daily rehabilitation program.

"I was still in the middle of my rehabilitation process. So, then it's quite easy, like "okay it's an investigation". At a certain point it was just scheduled in my day schedule, so it is just part of your day." (P12)

"A moment of relaxation! Hahaha, in here you walk from pillar to post and sometimes you have very intensive treatments and this - this was just a tranquil moment. You didn't have to do anything myself..." (P10)

The fact that they had a moment of relaxation and had the freedom to do nothing was appreciated by them.

Regarding the number of treatment sessions, patients indicated that if there had been a need from the trial or if it would have a (more) beneficial effect, they would have participated in more sessions. Patients also saw the treatment sessions as additional therapy: "The more [therapy], the better. Extra therapy, and just another step closer to perhaps an aid or an improvement, recovery." (P12) The positivity around additional therapy (implicitly) reflects the patient's belief that 'more therapy is better outcome'. Some patients indicated that their daily program was quite busy, which caused fatigue, however the B-STARS did not cause additional fatigue.

\section{$1.1 \mathrm{~g}$ No unpleasant aspects}

Overall, the patients commented favorably on the painless aspect of the treatment: "Yes, because it didn't hurt either. See, if it hurts then it's different, but ... it didn't hurt." (P4) Next to the painless treatment, the absence of negative consequences made them feel optimistic. One patient mentioned the non-invasive aspect of the brain stimulation. The pulses were often described by the patients in a neutral way or it was indicated that you had to get used to it. 


\subsection{Concerns}

\section{2a Effects of stimulation of the brain}

Some patients initially felt a bit nervous or anxious before the start of the treatment sessions. Patients were concerned about the 'electricity' within the brain and were unsure whether there would be negative consequences for the brain from the brain stimulation.

"It did create some tension. Normally I am not a person who experiences tension that easily, but this created a bit of tension, because I still consider it [rTMS] as electronics, and it is my own brain. The most important part of your body, so to speak. And I was thinking: 'I hope everything goes just right'. "(P7)

Some patients did not completely understand the relation between the diagnostic pulses and responses in one of the hand muscles during determination of the RMT, resulting in feelings of confusion.

"In the beginning I thought: 'hey that stings'. You know. I could not immediately make the connection. Those shocks came and I thought 'oooh, okay?'." (P1)

Others reported feelings of annoyance caused by the pulses.

"Well at a certain point if you have to continue for longer, then you will get kind of tired of it. But it doesn't really hurt. You can feel it more clearly around your temples than on your head. But when they navigate more to your temples, then you feel it more clearly. But still it doesn't hurt or so. It's just a bit annoying. It is not really pleasant." (P2)

\section{2b Equipment (chair and coil)}

Most patients expressed concerns about the chair where they had to sit in during the treatment. Comfort during the treatment was experienced as important. One patient was hindered by lack of support from the head rest and arm rests being too small.

"If I would have designed [the chair] for the person lying in it... I would have provided more support at the bottom of the neck, which makes it more comfortable. And I would make the handrail 2 centimeters wider so it is easier to place your arm on it." (P7)

Another patient said "The only downside I can mention is the chair in which I had to sit. I found it quite uncomfortable to sit back and then have to tilt my head back. Because of that chair, I was sitting in such a way that it wasn't a pleasant position, so you kind of freeze in a very uncomfortable position." (P8) 
This patient also indicated that longer periods in the chair would not be feasible: "So it was good that it did not last any longer, because then I would not have been able to keep up with it." (P8) Other patients also found the chair uncomfortable, but were not bothered by it because they were in the chair for only a short time and understood that everyone should fit in the chair.

Two patients had specific concerns regarding the coil "Sometimes you just had an uncomfortable hairpin on your head, so sitting in the chair was a little less comfortable, but that's all I have to say." (P10), and glue from the electrodes: "I don't like the glue... I mean not removing it, because it isn't a band-aid. It just remains sticky for a long time." (P5) used during the treatment sessions.

\title{
1.2c Logistics
}

Three patients expressed that ten treatment sessions are probably enough, since no recovery would be expected beyond those sessions, because they experienced no improvements in arm function during the intervention period. Or they indicated that the uncomfortable position in the chair would be a reason to exclude additional treatment sessions. One of them would not mind to receive fewer treatment sessions: "Shorter yes, but not longer." (P1) Patients experienced little or no fatigue due to the format of the therapy, but did express that the full day program of rehabilitation caused fatigue or concerns from nurses about their program.

\begin{abstract}
"The nursing staff gave me the impression that they thought that they thought it was a bit too much and a bit busy. But hey, according to me it fitted into my program and then you have to re-schedule a bit and it may mean that you had to take a shorter break occasionally." (P10)
\end{abstract}

\section{Participation in an RCT}

\subsection{General experience of recovery}

The most commonly perceived changes after the treatment were improvements in arm function. In some cases, the patients described their improvements in comparison to their initial paresis or to other patients in the rehabilitation facility.

"It [arm, body] was no longer paralyzed on the right side." (P5)

"Yes! When I see people who also attend the hand therapy group, but don't participate in the trial, I have made great progress." (P3)

Some patients felt that the improvements had led to noticeable impacts on daily life activities. Activities and participation seem to be major factors in patient's lives. 
"[I] put on my socks, put on my shoes. This morning I showered myself. I just have to be careful not to slip, but I do everything by myself. Combing my hair is going okay, but this part of my hair is still difficult. And I am able to shave myself with a razor blade and shaving cream, and you see I made no cuts." (P7)

"I can talk normally, I can walk, I am here by bike, I can drive, I can work, I can drink beers, hey I can basically do everything, except give people a good hand shake." (P12)

In most cases, the patients noted specific improvements, as a return of strength and speed, improved fine motor skills, being able to move upper extremity elements and performing better at the motor function tests.

\subsection{Experienced psychological effects}

Experienced psychological effects (i.e. benefits and concerns) of participation in an RCT were often raised by the patients, which we divided in the subthemes 'grateful', 'sense of purpose', 'recovery as a motivator', and 'group allocation'.

\section{2a Grateful}

Patients had the feeling to improve, and explicitly acknowledged the power of placeboeffect.

"Yes ... When I think it has worked, then it has worked. Yes, it's that simple.

You are not sure whether it has worked, but as long as you think it has worked, then it will at least have that effect." (P8)

It was evident in the transcripts that a number of patients felt gifted or as 'being chosen' when selected for the trial.

"I said now an angel should come to help me. And the door opens... and then actually a blonde angel comes in. And she asks if I want to participate in the program. I thought that must come from God, there is no other possibility. I am serious, it really experienced it that way. So, I told my wife that I should definitely participate, because there is no other way. So, it may sound strange, because I don't go to church or anything like that, but it really felt as a gift from God to me, and if I remember I might have said it too." (P7)

\section{2b Sense of purpose}

Participating in the intervention gave almost all patients a sense of purpose. On one hand, patients had a sense of helping themselves, e.g. working for maximum recovery. "I wanted it 
to be as soon as over, you know. And yes, I just wanted to get rid of it as quickly as possible. And yes, then the best thing I can do is by participating." (P1) On the other hand, patients stated that their contribution might benefit and can create a better future for other stroke patients, and they found this rewarding. "I want to contribute to the research because it might help others. That is the only reason." (P13) In addition to the sense of helping oneself and others, being able to contribute to science was also purposeful for many patients.

\section{2c Recovery as extra motivation to exercise}

Regardless whether patients improved because of receiving the rTMS treatment, noticing progress works as a great motivation. Performing the motor tests periodically, as part of their participation in the clinical trial, was appreciated by the patients. It felt like a feedback moment on the progress of their recovery, allowing improvement to be noticed by comparison with the previous performance on the test.

"So, then you notice that there is progress, I still have some progress so that also gives you motivation to continue." (P11)

Those quotes hint at the possibility they were more willing to put more effort in their rehabilitation than before the improvements. For some patients experiencing recovery contributed to a more positive mindset and future.

"Yes! You get a kind of boost, because you hope that it will help. You go into your next rehabilitation [session] with a more pleasant and relaxed feeling. In your daily schedule, you might think 'well, maybe it helps?' You do take the positive feeling to the next one [session]." (P2)

One patient expressed to be proud on the improvements made this far: "And I have to admit I am very proud that it is going so well." (P7)

\section{2d Disappointment and hope of group allocation}

A few patients indicated that allocation to the sham group would have disappointed them, suggesting that they were hoping for personal advantage. Despite the expressed disappointment of possible allocation to the control group, they did not drop out or refuse to participate.

\subsection{Motivation to participate}

\section{3a Personal benefit and cognitions}

All patients joined the study hoping that the treatment would positively impact their arm function (recovery). 
"It is the research that you can contribute to and if you are lucky and fall into the right group, then it may turn out to be positive. That is enough motivation for me to do something. Certainly because you know that the most progress can be expected in the first three months. And it now falls within those first three months. It seems to be highly recommendable to me." (P8)

This patient also expressed the hope to be in the intervention group, to be able to have a chance on recovery. Most patients had the belief that the brain can be influenced from outside, for example by using non-invasive brain stimulation. One patient could not make up his mind: "I do not know. Maybe. I do not actually know. I hope so, but I don't know for sure." (P4) Different arguments were brought up why or how placebo might work.

A belief in the expertise of the researchers and the expectation that patients will not be harmed also contributed to volunteering in the study. Multiple patients felt that "If you start with something, you need to finish it" (P9) which ensured constant motivation during the trial.

Several patients expressed that participation was an offer too good to refuse. "If you get the chance to do something like this and you can help someone forward with it, I take that chance. It doesn't cost a dime! Take that chance." (P7) Linked to this, patients also felt motivated by the following beliefs: "It doesn't hurt to try" and "Nothing ventured, nothing gained." The concept of karma was also described by one of the patients: "You always get it [friendliness] in return if you act friendly." (P7)

Most patients described that their attitude towards rehabilitation and recovery is of importance for the outcome.

"I tried to stay reasonably positive. Because yes it was made clear, that the way you look at things, determines what your recovery will look like." (P12)

"But if you immediately say no, then nothing happens either..." (P4)

A positive mindset and openness to experience seem ingredients for a fruitful recovery according to those patients.

Undergoing the treatment provided patients with an opportunity to be involved in scientific research, which they find fascinating. Their curiosity was fed.

\section{3b Altruism}

One of the most common reasons for participation in the trial was the hope to help current and future stroke patients. Several patients explicitly described their participation might benefit offspring or grandchildren. 
"Well, I hope that I can offer my children and granddaughter a longer life through this. Maybe ... the benefit, that when having a stroke, or whatever you call it, that intervention is possible in a different way than what is now. Because I saw it as extra therapy and I have done science a service and I hope to my offspring too. I am happy that I have been able to contribute to the research; for my offspring and the rest of the people." (P5)

This quote reflects the prevailing norms and values that are of importance to that person. Patients also demonstrated the willingness to participate to the advancement of science. Participation in the intervention provided patients with an opportunity to actively contribute to scientific research. One patient gave the example that participating would increase the diversity of age in the trial:

"... most people with whom I have been here are 60+. So, I thought maybe it is good, or interesting or nice for you ... that also a younger person, I was 30 when it happened. That you can also see what it does to a young person. Perhaps there were very different results with me than with a fellow patient who is already in his seventies or sixties." (P12)

\section{Discussion}

The aim of this qualitative study was to gain insight into how rTMS treatment for upper limb recovery within stroke rehabilitation was experienced by patients who participated in the clinical trial. Overall, the patients reported positive experiences with the TMS treatment and believed that receiving rTMS had benefited their arm function. Aspects of the treatment that were experienced most favorably were the comfort of the treatment setting (e.g. moment of relaxation) and the absence of pain and side-effects. The main concerns were the fear of or uncertainty about negative consequences from the electrical currents within the brain and the annoyance of the pulses on the head. Most patients participated in the study to contribute to knowledge for treatment of future stroke patients and for personal benefit. Participation in a clinical trial was also experienced as a grateful experience and gave patients a sense of purpose.

\section{Benefits and concerns related to rTMS treatment}

Patients felt that the rTMS treatment was effective for the recovery of their affected arm, even though they were blinded from the treatment assignment -which could be sham stimulation- and outcome. Effectiveness was described by patients as being able to perform fine and gross movements with the affected arm (i.e. reduction of impairment 
and disability), being able to wash and dress themselves, and being able to participate in traffic as cyclist or car driver (i.e. activity and participation). These outcomes are contrary to the only (known) previous qualitative study in the field of non-invasive brain stimulation of Triccas et al., ${ }^{14}$ where patients felt that receiving tDCS and robotic therapy was especially effective for their strength and tightness in the affected upper limb. This discrepancy in results could be explained by the design of the studies. Triccas et al. ${ }^{14}$ used a robot, paired with tDCS, which trains hand grip rather than hand movements. The lack of ability to carry out two-handed tasks easier was one of the main concerns reported by their patients. The expressed hope and expectations of personal benefit for recovery are consistent with the main reasons for participation stated by patients in our trial. Assumptions of therapeutic benefit from participation in (stroke rehabilitation) research has also been found and discussed in other studies. . $^{23,24}$

The included patients evaluated receiving rTMS treatment positively. Positive experiences arose from the comfortable treatment settings, the interaction with the research-therapists, the way of information delivery, learning experiences, and the absence of pain or negative consequences. Comfort of the treatment was related to the way the treatment was built into their daily schedule, the low burden of the treatment procedure (i.e. maximally five minutes; comfortable position in the treatment chair), and the setting in which they received the brain stimulation. Patients were pleased that the treatments were scheduled and they experienced the treatments as a moment of relaxation during their full and sometimes tiring daily rehabilitation program. The short duration of the treatments may have ensured that the patients did not experience the intervention as a burden. However, in a qualitative descriptive study a high intensity programme was not viewed as a barrier to engagement for the included stroke patients. The acceptability and engagement with the high intensity programme was mediated by several factors, including making progress, internal and external motivators, and other group members. ${ }^{25}$ The treatment room in our study, which was experienced as quiet and low on stimuli, also contributed to the relaxed experience. The importance of comfort has also been emphasized by patients who evaluated novel stroke technologies. ${ }^{26}$

The interaction with research staff administering the treatment was highly valued among the patients. Attributes of the researchers, i.e. being calm, competent, transparent and respectful, enabled a sense of safety and a strong therapeutic relationship. The friendly attitude of the research staff was highly valued and made patients look forward to their treatment session. These findings agree with results from a qualitative study ${ }^{27}$ that reported that to be treated with respect and dignity was the core factor contributing to elderly stroke patients' satisfaction with rehabilitation therapy. Subcategories as being treated with humanity, having confidence and trust in professionals, and dialogue and exchange of information were other determinants of satisfaction. ${ }^{27}$ In our study, the clarity of information 
and the step-by-step explanation of the different actions ensured that patients knew what to expect, and made them feel safe. The desire for information has previously been recognized as a key coping strategy and as a resource for psychosocial adjustment and empowerment. 27,28

However, in addition to reported feelings of therapeutic benefit and positive experiences of undergoing rTMS treatment, some concerns were also identified. One of the main concerns were feelings of anxiety about the 'electricity' within the brain and uncertainty about possible negative consequences. This finding is consistent with findings from Triccas et al. ${ }^{14}$ who reported that patients undergoing tDCS treatment had concerns about the 'electricity' applied via the electrodes, and were insecure about possible negative consequences. Some patients found the stimulation pulses annoying. The somatic scalp sensation due to TMSinduced activation of superficial nerves or muscles might feel different for each person. ${ }^{29}$ The reported annoyance of the TMS pulses, however, appear less severe compared to the reported itchy, painful, and burning sensations after tDCS. ${ }^{14}$ The therapeutic potential of TMS is not limited to stroke patients, as efficacy, tolerability and safety have also been demonstrated for other neurological and psychiatric disorders. ${ }^{9}$ TMS has been clinically approved for treatment of major depressive disorder, for which there are comprehensive guides for the safe administration of TMS while ensuring patient comfort (e.g. with regard to head support or cushioning lighting and room temperature, and small adjustments in the placement or rotation of the coil). ${ }^{30}$

\section{Benefits and concerns related to participation in an RCT}

The beneficial psychological impact of trial participation, beyond those of the treatment, has been increasingly recognized. ${ }^{31,32}$ Patients who perceived recovery during participation in our trial found this hopeful and motivating, which made them more likely to put effort in their rehabilitation. Our findings are consistent with data from studies that investigated stroke survivors' perspective of upper limb recovery after stroke. ${ }^{33-38}$ An individual's experience of recovery helps to maintain their motivation. ${ }^{33,34}$ Furthermore, the hope for and belief in further recovery is related to an individual's attempt to maximize upper limb recovery (i.e. 'keeping doors open') and overall stroke recovery. ${ }^{35,37,38}$

Patients reported a powerful belief that they might receive an effective treatment, and acknowledged the possible effects on health and well-being. Expectancy, what a patient thinks or expects to happen as a result of the treatment, is one of the many contributing factors to the recognized placebo response. ${ }^{39}$ In addition to a placebo effect, being offered or 'chosen' to participate in a trial, resulting in a sense of hope, might contributed positively to emotional well-being. Trial participation as a privilege has also been reported by patients enrolled in a 
cancer-related clinical drug treatment trial. ${ }^{40}$ In our study, trial participation was also associated with the satisfaction of feeling useful and having a sense of purpose. Those feelings derived from being able to actively contribute to one's recovery process, contributing to help for current and future stroke patients, and being part of scientific research resulting in more knowledge and therapeutic advancements. Curiosity and interest in scientific research were also mentioned as reasons to participate. By participating in the intervention, patients could learn more about the (working of the) brain and experience a novel rehabilitation technique. The societal benefits of participating in a trial and contributing to something greater than yourself, which may be consistent with existential well-being, has been reported for patients before. ${ }^{31,41}$ The concept of "intergenerational altruism" has been introduced to describe altruistic motivations to participate in research for the benefit of younger generations. ${ }^{42}$

Some patients shared concerns about allocation to the control group, which could limit their chance of additional improvement. Several studies have explored the psychological burden for patients participating in an RCT, who were informed by the result of randomization, and acknowledged the discomfort of being randomized, and anxiousness or embarrassment to receive placebo. ${ }^{23,43}$ However, in our study the patients were unaware of group allocation. The concept of random allocation might be more difficult to accept for patients who know that they are receiving placebo, because they can compare themselves to the non-placebo group.

\section{Limitations}

Our study has some limitations. First, the interviews were not conducted at a fixed time after the intervention period, causing some patients to have to recall memories from one to two years ago. Difficulties in recalling experiences could introduce a risk of cognitive bias. However, even in patients who were interviewed one to two years after participation in the trial, we noticed that they could reproduce their memories, suggesting that a period to process and reflect upon your experiences could also lead to a more matured memory. Second, the first author designed the protocol, recruited patients for the RCT, and analyzed the qualitative data, introducing the risk of bias. To minimize this risk, the author (LJ) who undertook the interviews and took no part in the RCT, also assisted in the data analysis. Third, although representative for many European hospitals, the rehabilitation setting in which our study was conducted may be different from other healthcare situations.

\section{Clinical implications and directions for future research}

Several treatment characteristics that could lead to effective implementation of rTMS in clinical care and implications of the findings for inpatient stroke rehabilitation and recommendations for future rTMS trials were identified from the interviews. 


\section{Implementation in clinical care}

According to the experiences of the patients in an inpatient facility it is feasible to add rTMS therapy to a daily inpatient stroke rehabilitation program. Undergoing this treatment was not experienced as a burden. Careful communication seems to be a key factor in a patient's sense of safety and satisfaction with rTMS treatment participation. Transparency, explanation of the procedures in layman's terms, and a humane approach to the patient seem to be important factors. To anticipate to the patient's concerns about electrical currents in the brain, negative consequences and painful sensations, personnel should pay attention to recurrently explaining the mode of action of rTMS, the non-invasive character of the treatment, and the limited possible risks. It is essential to pay close attention to the settings (i.e. equipment use, support materials, environment) in which the treatment is delivered to prevent discomfort. The active involvement of patient's perspectives in the design of rTMS treatment delivery could be central in this.

\section{Inpatient stroke rehabilitation}

In the present study, we identified that patients value a moment of relaxation in between appointments of their rehabilitation program. These moments of rest could be built into the standard routine of inpatient stroke rehabilitation. According to the patients, the relaxation was felt because they could sit in a comfortable declined chair in a quiet room, and had the freedom to do nothing. The full procedure of preparation, installation and treatment delivery took a maximum of 10 minutes (the actual rTMS treatment lasted only 40 seconds), showing that this effect can be achieved in a relatively short period of time.

We also found that patient value feedback on their progress of motor recovery, as it contributes to an individual's expectation, motivation, and attempts to maximize upper limb recovery. This could include communicating repeated measurements to the patient in advance and the use of patient reported outcome measures (PROMs). Repeating measurements over time and at specific time-points post-stroke (i.e. at least three months stroke, according to the $\mathrm{SRRR}^{44}$ ) is relevant for clinical care as research.

\section{Future rTMS trials}

Our results emphasize that participation in scientific research makes people feel useful and empowers them to contribute to something bigger than themselves. Future rTMS trials should consider describing the positive effects of participation in scientific research in their patient information letter. In addition, involved stakeholders (e.g. nursing staff, facility personnel) should also be aware of the positive effects that patients can experience in trial participation, especially when there are doubts about the feasibility of a study in rehabilitation care. The patient information letter should also clearly explain the working of the TMS and the possible side-effects. 


\section{Conclusions}

The present qualitative study provides critical insights in how the design and delivery of rTMS treatment for upper limb recovery is experienced by patients. The acquired information can aid in improvement of the design of future rTMS trials and implementation in routine stroke rehabilitation programs. rTMS was well accepted and even enjoyed by patients. Comfortable treatment settings, respectful communication, and transparent information contributed to the experienced satisfaction. The experiences and preferences of stroke patients could be useful in the design of future rTMS studies and the implementation of rTMS in clinical care.

\section{Acknowledgements}

We thank the patients who participated in this trial.

\section{References}

1. Go, A. et al. Heart Disease and Stroke Statistics-2013 Update: A Report From the American Heart Association. Circulation 127, e36-e245 (2013).

2. Morris, J. H., Van Wijck, F., Joice, S. \& Donaghy, M. Predicting health related quality of life 6 months after stroke: The role of anxiety and upper limb dysfunction. Disabil. Rehabil. 35, 291-299 (2013).

3. Franceschini, M., La Porta, F., Agosti, M. \& Massucci, M. Is health-related-quality of life of stroke patients influenced by neurological impairments at one year after stroke? Eur. J. Phys. Rehabil. Med. 46, 389-399 (2010).

4. Houwink, A., Nijland, R. H., Geurts, A. C. \& Kwakkel, G. Functional recovery of the paretic upper limb after stroke: Who regains hand capacity? Arch. Phys. Med. Rehabil. 94, 839-844 (2013).

5. Kwakkel, G., Kollen, B. \& Lindeman, E. Understanding the pattern of functional recovery after stroke: facts and theories. Restor. Neurol. Neurosci. 22, 281-299 (2004).

6. Zhang, L. et al. Low-Frequency Repetitive Transcranial Magnetic Stimulation for Stroke-Induced Upper Limb Motor Deficit: A Meta-Analysis. Neural Plast. 2017, 1-12 (2017).

7. van Lieshout, E. C. C., van der Worp, H. B., Visser-Meily, J. M. A. \& Dijkhuizen, R. M. Timing of Repetitive Transcranial Magnetic Stimulation Onset for Upper Limb Function After Stroke: A Systematic Review and Meta-Analysis. Front. Neurol. 10, 1-16 (2019).

8. Liew, S., Santarnecchi, E., Buch, E. \& Cohen, L. Non-invasive brain stimulation in neurorehabilitation: local and distant effects for motor recovery. Front. Hum. Neurosci. 8, 1-15 (2014).

9. Lefaucheur, J. P. et al. Evidence-based guidelines on the therapeutic use of repetitive transcranial magnetic stimulation (rTMS). Clin. Neurophysiol. 131, 474-528 (2020).

10. Kubis, N. Non-Invasive Brain Stimulation to Enhance Post-Stroke Recovery. Front. Neural Circuits 10, 56 (2016).

11. Chung, S. W., Hill, A. T., Rogasch, N. C., Hoy, K. E. \& Fitzgerald, P. B. Use of theta-burst stimulation in changing excitability of motor cortex: A systematic review and meta-analysis. Neurosci. Biobehav. Rev. 63, 43-64 (2016).

12. Klomjai, W., Katz, R. \& Lackmy-Vallée, A. Basic principles of transcranial magnetic stimulation (TMS) and repetitive TMS (rTMS). Ann. Phys. Rehabil. Med. 58, 208-213 (2015). 
13. Kakuda, W. et al. Combination Protocol of Low-Frequency rTMS and Intensive Occupational Therapy for Post-stroke Upper Limb Hemiparesis: a 6-year Experience of More Than 1700 Japanese Patients. Transl. Stroke Res. 7, 172-179 (2016).

14. Tedesco Triccas, L. et al. A qualitative study exploring views and experiences of people with stroke undergoing transcranial direct current stimulation and upper limb robot therapy. Top. Stroke Rehabil. 9357, 1-9 (2018).

15. Kirsch, I. Response expectancy as a determinant of experience and behavior. Hypn. Theory, Res. Appl. 199-212 (2017). doi:10.4324/9781315252858-20

16. Evers, A. W. M. et al. Implications of placebo and nocebo effects for clinical practice: Expert consensus. Psychother. Psychosom. 87, 204-210 (2018).

17. Peerdeman, K. J. et al. Relieving patients' pain with expectation interventions: A meta-analysis. Pain 157, 1179-1191 (2016).

18. Van Lieshout, E. C. C., Visser-Meily, J. M. A., Neggers, S. F. W., Van Der Worp, H. B. \& Dijkhuizen, R. M. Brain stimulation for arm recovery after stroke (B-STARS): Protocol for a randomised controlled trial in subacute stroke patients. BMJ Open 7, 1-10 (2017).

19. Huang, Y. Z., Edwards, M. J., Rounis, E., Bhatia, K. P. \& Rothwell, J. C. Theta burst stimulation of the human motor cortex. Neuron 45, 201-206 (2005).

20. Collin, C. \& Wade, D. Assessing motor impairment after stroke: A pilot reliability study. J. Neurol. Neurosurg. Psychiatry 53, 576-579 (1990).

21. Braun, V. \& Clarke, V. Using thematic analysis in psychology. Qual. Res. Psychol. 3, 77-101 (2006).

22. Hennink, M. M., Kaiser, B. N. \& Marconi, V. C. Code Saturation Versus Meaning Saturation: How Many Interviews Are Enough? Qual. Health Res. 27, 591-608 (2017).

23. Norris, M., Poltawski, L., Calitri, R., Shepherd, A. I. \& Dean, S. G. Hope and despair: A qualitative exploration of the experiences and impact of trial processes in a rehabilitation trial. Trials 20, 1-11 (2019).

24. Appelbaum, P. S., Roth, L. H., Lidz, C. W., Benson, P. \& Winslade, W. False Hopes and Best Data: Consent to Research and the Therapeutic Misconception. Hastings Cent. Rep. 17, $20-24$ (1987).

25. Signal, N. et al. What influences acceptability and engagement with a high intensity exercise programme for people with stroke? A qualitative descriptive study. NeuroRehabilitation 39, 507-517 (2016).

26. Meadmore, K. L., Hughes, A.-M., Freeman, C. T., Benson, V. \& Burridge, J. H. Participant Feedback in the Evaluation of Novel Stroke Rehabilitation Technologies. J. Rehabil. Robot. 1, 82-92 (2013).

27. Harrison, M., Ryan, T., Gardiner, C. \& Jones, A. Psychological and emotional needs, assessment, and support post-stroke: A multi-perspective qualitative study. Top. Stroke Rehabil. 24, 119-125 (2016).

28. Lambert, S. D. \& Loiselle, C. G. Health Research Health Information - Seeking Behavior. Qual. Health Res. 17, 1006-1019 (2007).

29. Lefaucheur, J. P. et al. Evidence-based guidelines on the therapeutic use of repetitive transcranial magnetic stimulation (rTMS). Clin. Neurophysiol. 125, 2150-2206 (2014).

30. Van Trees, K. et al. Comprehensive Guide for the Safe Administration of rTMS While Providing for Patient Comfort. Issues Ment. Health Nurs. 38, 182-187 (2017).

31. Harrop, E. et al. 'I didn't really understand it, I just thought it'd help': Exploring the motivations, understandings and experiences of patients with advanced lung cancer participating in a nonplacebo clinical IMP trial. Trials 17, 1-12 (2016).

32. Braunholtz, D. A., Edwards, S. J. L. \& Lilford, R. J. Are randomized clinical trials good for us (in the short term)? Evidence for a 'trial effect'. J. Clin. Epidemiol. 54, 217-224 (2001).

33. Mangset, M., Dahl, T. E., Forde, R. \& Wyller, T. B. We're just sick people, nothing else': ... factors contributing to elderly stroke patients' satisfaction with rehabilitation. Clin. Rehabil. 22, 825-835 (2008). 
34. Hole, E., Stubbs, B., Roskell, C. \& Soundy, A. The patient's experience of the psychosocial process that influences identity following stroke rehabilitation: A metaethnography. Sci. World J. 2014, 349151 (2014).

35. Wiles, R., Ashburn, A., Payne, S. \& Murphy, C. Patients' expectations of recovery following stroke: A qualitative study. Disabil. Rehabil. 24, 841-850 (2002).

36. Barker, R. N., Gill, T. J. \& Brauer, S. G. 'Factors contributing to upper limb recovery after stroke: A survey of stroke survivors in Queensland Australia'. Disabil. Rehabil. 29, 981-989 (2007).

37. Luker, J., Lynch, E., Bernhardsson, S., Bennett, L. \& Bernhardt, J. Stroke Survivors' Experiences of Physical Rehabilitation: A Systematic Review of Qualitative Studies. Arch. Phys. Med. Rehabil. 96, 1698-1708.e10 (2015).

38. Barker, R. N. \& Brauer, S. G. Upper limb recovery after stroke: The stroke survivors' perspective. Disabil. Rehabil. 27, 1213-1223 (2005).

39. Kaptchuk, T. J. \& Miller, F. G. Placebo effects in medicine. N. Engl. J. Med. 373, 8-9 (2015).

40. Wootten, A. C., Abbott, J. M., Siddons, H. M., Rosenthal, M. A. \& Costello, A. J. A qualitative assessment of the experience of participating in a cancer-related clinical trial. Support. Care Cancer 19, 49-55 (2011).

41. Balfour, L. et al. Altruism motivates participation in a therapeutic HIV vaccine trial (CTN 173). AIDS Care - Psychol. Socio-Medical Asp. AIDS/HIV 22, 1403-1409 (2010).

42. Mein, G. et al. Altruism and participation in longitudinal health research? Insights from the Whitehall II Study. Soc. Sci. Med. 75, 2345-2352 (2012).

43. Naidoo, N. et al. The research burden of randomized controlled trial participation: A systematic thematic synthesis of qualitative evidence. BMC Med. 18, 1-11 (2020).

44. Kwakkel, G. Standardised measurement of sensorimotor recovery in stroke trials: consensus-based core recommendations from the Stroke Recovery and Rehabilitation Roundtable (SRRR). Int. J. Stroke 12, 451-461 (2017).

45. Brott, T. et al. Measurements of acute cerebral infarction: A clinical examination scale. Stroke 20, 864-870 (1989).

46. Collin, C., Wade, D. T., Davies, S. \& Horne, V. The barthel ADL index: A reliability study. Disabil. Rehabil. 10, 61-63 (1988).

47. Spinhoven, P. et al. A validation study of the Hospital Anxiety and Depression Scale (HADS) in different groups of Dutch subjects. Psychol. Med. 27, 363-370 (1997).

48. Duncan, P. W., Propst, M. \& Nelson, S. G. Reliability of the Fugl-Meyer assessment of sensorimotor recovery following cerebrovascular accident. Phys. Ther. 63, 1606-1610 (1983). 


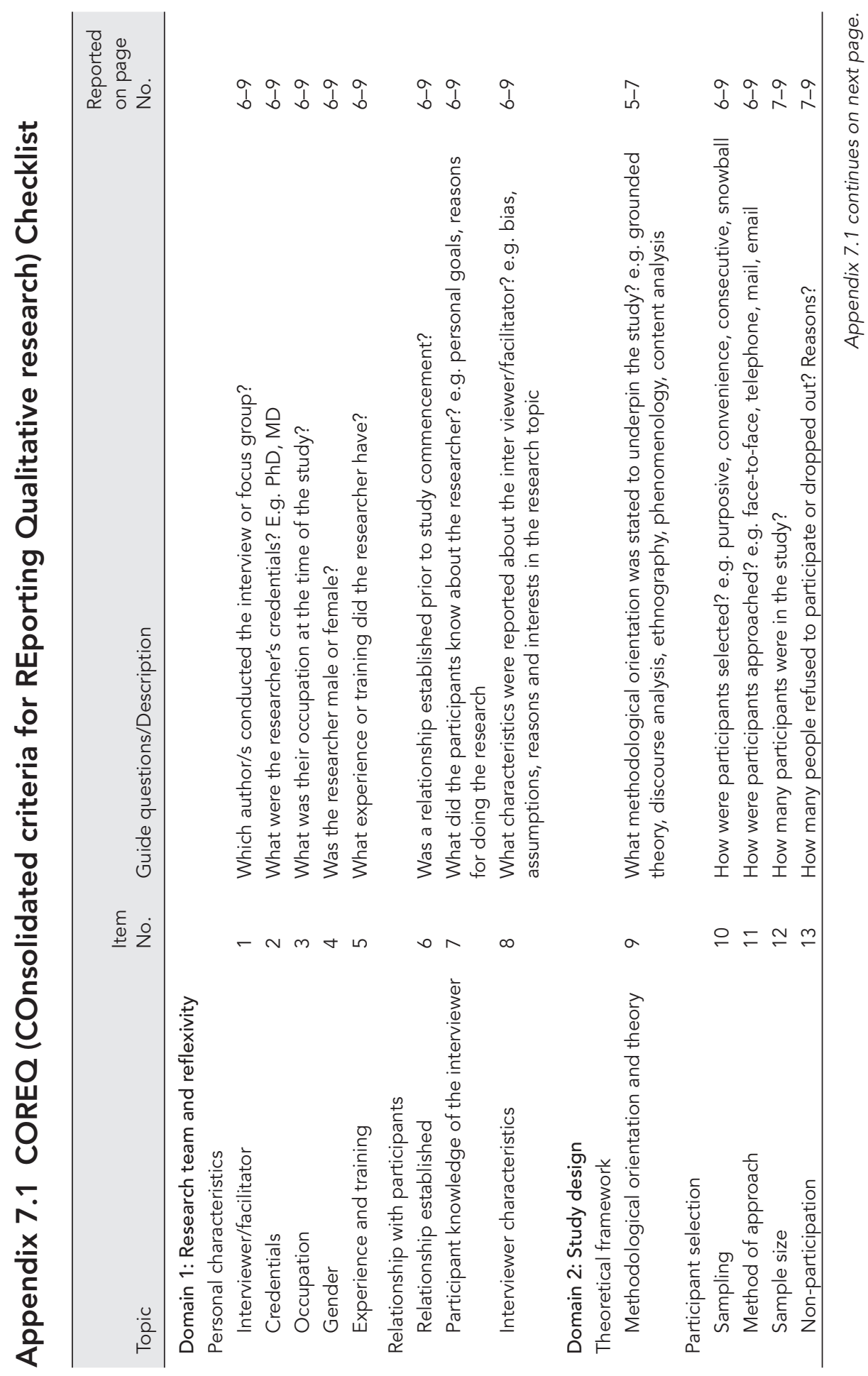




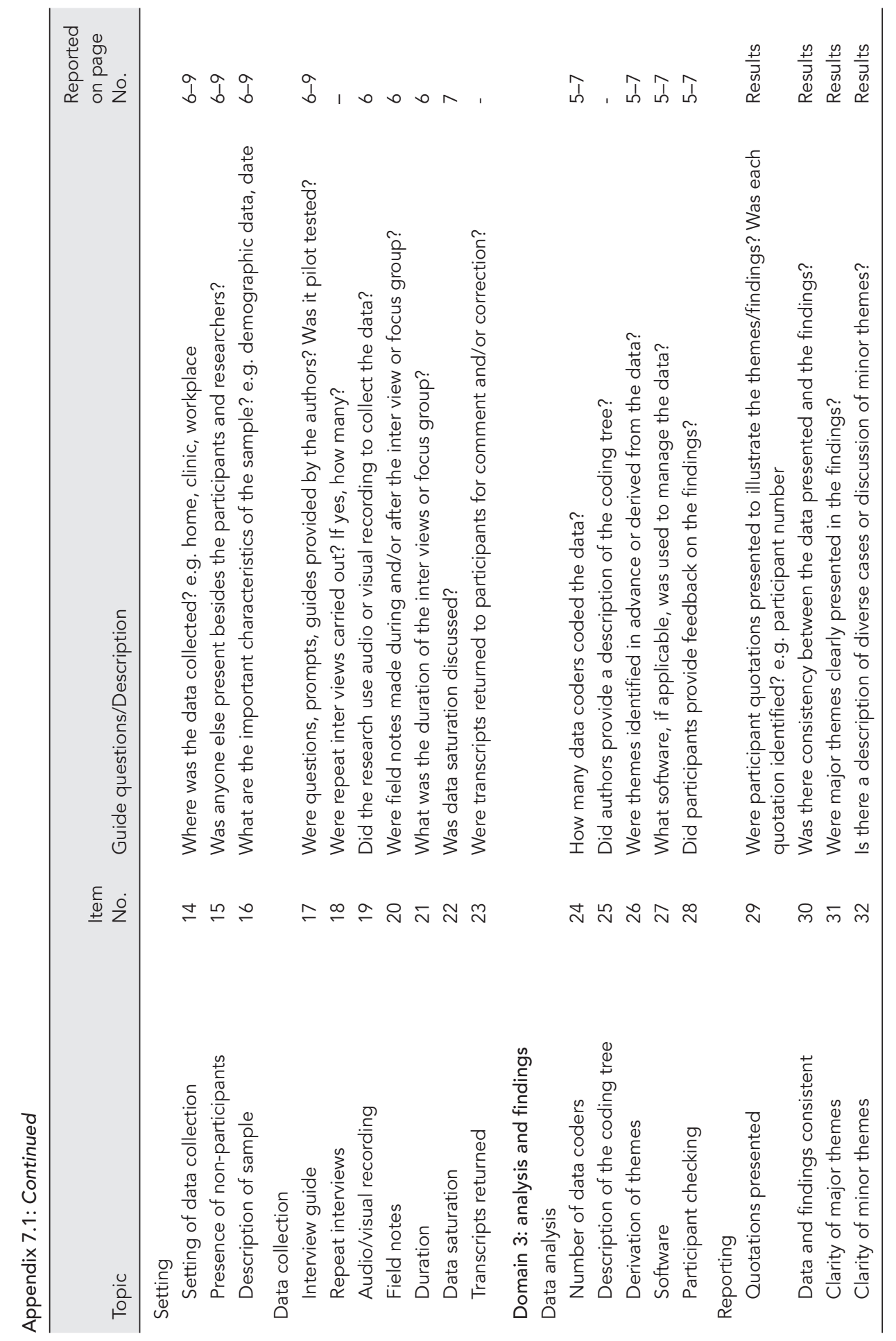




\section{Appendix 7.2 Interview guidelines}

\section{Topics interviews}

- General experience with the brain stimulation treatments

- Perceived pleasant aspects of the treatment

- Perceived unpleasant aspects of the treatment

- Motivation for participating and completing the program

- Emotions experienced by the participant during different stages of the program:

- Before start of the treatments

- During the treatments

- As the treatment progress

- Experiences with the different sensations/elements of the treatments:

- Coil

- Electrodes

- Wrist band

- Pulses

- Chair

- Physical sensations

- Perceived benefits of the program

- Effectivity of the treatments

- How are the participants since they completed their treatments?

- Perceived disadvantages of the program

- Duration of the treatments

- Recommendation to other stroke patients

- Experiences with the arm training

- Experiences with information from the research(ers):

- Before start of the treatments

- During the treatments

- Clarity of the information

- Understanding what might be expected from the participants

- General cognitions of the participants:

- Expectations/cognitions about brain stimulation before start treatments

- Expectations/cognitions about brain stimulation after completing the treatments

- Opinions about the developments of new treatment techniques

- Working of the brain

- Thoughts about how the treatments fit in their regular program

- Intensity of participation

- Points of improvements from the participants 


\section{Appendix 7.3 Checklist of Criteria for Good Thematic Analysis: 15-point checklist}

\begin{tabular}{|c|c|c|}
\hline Process & Criteria & $\begin{array}{l}\text { Reported } \\
\text { in chapter }\end{array}$ \\
\hline Transcription & $\begin{array}{l}\text { 1. The data have been transcribed with an appropriate level of } \\
\text { detail, and the transcripts have been checked against the tapes } \\
\text { for 'accuracy'. }\end{array}$ & YES \\
\hline \multirow[t]{5}{*}{ Coding } & $\begin{array}{l}\text { 2. Each data item has been given equal attention in the coding } \\
\text { process. }\end{array}$ & YES \\
\hline & $\begin{array}{l}\text { 3. Themes have not been generated from a few vivid examples } \\
\text { (an anecdotal approach), but instead the coding process has } \\
\text { been thorough, inclusive and comprehensive. }\end{array}$ & YES \\
\hline & 4. All relevant extracts for each theme have been collated. & YES \\
\hline & $\begin{array}{l}\text { 5. Themes have been checked against each other and against the } \\
\text { original data set. }\end{array}$ & YES \\
\hline & 6. Themes are internally coherent, consistent, and distinctive. & YES \\
\hline \multirow[t]{4}{*}{ Analysis } & $\begin{array}{l}\text { 7. Data have been analysed - interpreted, made sense of - rather } \\
\text { than just paraphrased or described. }\end{array}$ & YES \\
\hline & $\begin{array}{l}\text { 8. Analysis and data match each other - the extracts illustrate the } \\
\text { analytical claims. }\end{array}$ & YES \\
\hline & $\begin{array}{l}\text { 9. Analysis tells a convincing and well-organised story about the } \\
\text { data and topic. }\end{array}$ & YES \\
\hline & $\begin{array}{l}\text { 10. A good balance between analytical narrative and illustrative } \\
\text { extracts is provided. }\end{array}$ & YES \\
\hline Overall & $\begin{array}{l}\text { 11. Enough time has been allocated to complete all phases of the } \\
\text { analysis adequately, without rushing a phase or giving it a once- } \\
\text { over-lightly. }\end{array}$ & YES \\
\hline \multirow[t]{4}{*}{$\begin{array}{l}\text { Written } \\
\text { report }\end{array}$} & $\begin{array}{l}\text { 12. The assumptions about, and specific approach to, thematic } \\
\text { analysis are clearly explicated. }\end{array}$ & YES \\
\hline & $\begin{array}{l}\text { 13. There is a good fit between what you claim you do, and what } \\
\text { you show you have done - i.e., described method and reported } \\
\text { analysis are consistent. }\end{array}$ & YES \\
\hline & $\begin{array}{l}\text { 14. The language and concepts used in the report are consistent } \\
\text { with the epistemological position of the analysis. }\end{array}$ & YES \\
\hline & $\begin{array}{l}\text { 15. The researcher is positioned as active in the research process; } \\
\text { themes do not just 'emerge'. }\end{array}$ & YES \\
\hline
\end{tabular}

Adapted from: Braun, V., \& Clarke, V. Using thematic analysis in psychology. Qual. Res. Psychol.3, 77-101 (2006). 
General discussion 

This final chapter starts with an overview of the main findings and conclusions of the studies described in this thesis. First, I will focus on the understanding and assessment of poststroke motor impairment and recovery. Thereafter, I will discuss the pathways to optimal recovery and the use of non-invasive brain stimulation. Subsequently, implications for clinical practice are discussed. Finally, methodological considerations and ideas for future research are presented.

\section{The understanding and assessment of post-stroke motor impair- ment and recovery}

\section{Translational research}

Translational research in neuroscience involves, among others, extrapolation of results in animal studies to human neurological conditions, such as stroke. ${ }^{1-3}$ This type of research can have many objectives, of which investigating neural and behavioral changes following stroke, developing a restorative procedure, and translating the procedure to clinical trials and practice are noteworthy. ${ }^{2-4}$ Currently, we find that generalizations from translational research on stroke recovery are often limited, due to the lack of differentiation between true recovery and compensation and the inadequate interpretation of behavioural findings. ${ }^{1,4,5}$ As described in the Chapter 1 and Chapter 2, functional recovery occurs through either true recovery (so-called restitution of function) or compensation (so-called substitution of function). In addition, when recovery is evaluated without taking compensation into account, this could distort the understanding of neural plasticity and recovery after stroke. This may affect the results from basic neuroscience and translational research to guide treatment.6,7 Skilled reaching is a behavioral method that allows for a distinction between true recovery and compensation, and shows significant homologies between rats and humans. ${ }^{8,9}$ In order to maximize clinical translation of the skilled reaching task, we addressed how motor recovery patterns correspond between rat and human skilled reaching sub-acutely post-stroke, and determined the relation between skilled reaching and clinical outcome measures. As was shown in Chapter 2, rats and humans show similarities in skilled reaching performance over time. Both species show muscle flaccidity at the first measurement post-stroke followed by motor recovery according to a proximal-to-distal principle. Rats showed a return to (almost) normal movement for certain elements (i.e. limb lift, digits semi-flexed, pronation, and grasp) early after stroke, while humans exhibited a slower improvement on these elements over time. Nevertheless, these findings suggest that recovery of skilled reaching performance in general in rats is comparable to that of humans, allowing for useful generalizations.

The Stroke Recovery and Rehabilitation Roundtable (SRRR) published guidelines to enhance the alignment of the preclinical and clinical stroke recovery research pipelines. They 
recommend using comparable preclinical and clinical outcome measures to minimize the potential of translational failure. ${ }^{10,11}$ In terms of impairment-specific testing, sensitive tests are available to assess clinically relevant deficits, such as skilled reaching, hind- and forelimb impairments, and gait abnormalities. ${ }^{11,12}$ At this moment, there is no preclinical equivalent of the Fugl-Meyer Upper Extremity (FM-UE) scale, which is used in humans as an impairment measure. However, the first ideas have been put forward to design a preclinical counterpart of the FM-UE. ${ }^{12}$ We showed that skilled reaching in humans is strongly correlated to clinical outcome measures (ARAT and FM-UE) at different time-points during the first three months post-stroke (Chapter 2). The development of skilled reaching scores was also similar to the development of clinical outcome scores. Kinematic and kinetic measures might serve as the most suitable options to distinguish true recovery from compensation. ${ }^{13}$ However, there is no consensus yet on a core set of valid kinematic and kinetic measures. In addition, it requires an expensive set-up, and the measures are hard to perform and analyze, which also limits their use in clinical practice. ${ }^{12,13}$ Until there is an alignment in the methodology of impairment measurement in preclinical and clinical studies, it is important that preclinical research not only continues to use performance-based measures (e.g. success scores of pellet retrieval), but also includes analysis of the reaching pattern of rats after stroke, to improve the translation of preclinical findings to the clinic. Furthermore, skilled reaching might serve as an inexpensive tool to distinguish between recovery and compensation in clinical care.

\section{Patient-reported outcome}

Health information directly from patient themselves, so-called patient-reported outcome measures (PROMs), has received increasing attention across research, clinical care, and public health. ${ }^{14}$ The transition to value-based health care has led to a wider implementation of PROMs in stroke clinical care, because they are important in critically evaluating healthcare. ${ }^{15}$ The primary focus of most therapies is to improve symptoms, functional status, or HRQoL. These aspects of a patient's health status are best captured by a patient self-report, ${ }^{16}$ because the importance and meaningfulness of each outcome can vary from patient to patient. Furthermore, what is considered as a good outcome by a clinician may not be similarly rated by a patient or the family. ${ }^{16}$ As was shown in Chapter 3, responding to the debate around the clinical utility of PROMs, self-reported questionnaires used for monitoring upper limb recovery are accurate compared with observationally measured improvement in the early and late subacute phase after stroke. It is no longer necessary to question the use of PROMs for their accuracy regarding the reliance on self-evaluation. Furthermore, the timing of assessment post-stroke does not affect the accuracy of selfreports in the sub-acute stages, allowing PROMs to be reliably used repeatedly during the 6-month period after stroke. It is currently advised to collect PROMs data at discharge 
from hospital and at 90 days after admission or the index event. ${ }^{17}$ Therefore, I recommend an update on the advice to collect PROMs data at multiple time-points across the first 6 months and preferably up to 1 year post-stroke in the ICHOM International Standard Set of Patient-Centered Outcome Measures.

Despite self-reported measures being accurate, we did find some discrepancies between self-reported and observational measures, so-called mismatches. This provides us with additional information that can help to design optimized rehabilitation strategies (Chapter 3). This role for PROMs data on mismatches to inform and influence stroke care has never been investigated before. Patients with a mismatch between the measures, a self-reported underor overestimation of capacity, might need a different rehabilitation approach. For patients who underestimate their capacity, a focus should be on training in the use of the affected hand, positive psychology, self-efficacy, and expectation management. For the patients who overestimate their capacity, training in body image may be warranted. A patient's awareness of deficits is of significance for the rehabilitation process, especially for successful motivated participation and achieving rehabilitation goals. ${ }^{18} \mathrm{~A}$ lack of awareness may lead to poorer functional outcomes, because patients may not actively engage in therapeutic activities, do not recognize when they need help, and are unlikely to be motivated. ${ }^{19}$ The research we conducted provides answers to whether we can rely on the self-appraisal of functioning, as PROMs may be affected by confounding factors, such as neglect, self-awareness, mood, fatigue, social support, relationships, and encouragement from others. ${ }^{20-23}$

However, the accuracy of PROMs data does not solely depend on the correspondence between observational and self-reported data. For example, neuropsychological deficits may complicate or prevent patients from assessing PROMs, or require assistance from another person completing the measurement. ${ }^{24}$ Alternative data collection methods, such as visual versions of scales for aphasia patients, can reduce selection bias by not excluding patient who can't complete PROMs tests, because the use of proxies can introduce measurement error. Other practical challenges related to the collection of PROMs data are time constraints in clinical care, absence of standardized tools to assess the outcomes, and the use of technology (e.g. little access to computers, slow computer networks, lack of computer skills among staff). ${ }^{25,26}$ Besides user's guides for clinicians for implementing PROMs in clinical practice, and the development of a minimum recommended set of patient-centered outcomes for stroke patients, the stroke community will have to commit to further research on PROMs and their implementation in clinical care.

$\mathrm{HRQOL}$ is one of the PROMs that is frequently used in research and clinical care to evaluate a patient's health status. The majority of the HRQoL measures are stroke-specific, as stroke is known to impact domains that are not typically captured by generic HRQoL measures. 
Each patient experiences the degree to which symptoms and limitations of their disease have an overall impact on their well-being differently. Given the high prevalence of upper limb paresis after stroke, we assessed the degree and relative impact of the relationship between upper limb strength and HRQoL both cross-sectionally and longitudinally. Upper limb strength appears to be the most important predictor of HRQoL in the sub-acute phase after stroke, besides other predictors as leg function and anxiety (Chapter 4). Upper limb paresis compromises a stroke patient's capacity to participate in (meaningful) activities, since the upper extremities play a crucial role in daily activities. Furthermore, an improvement in upper limb strength was positively related with an improvement in $\mathrm{HRQOL}$, which suggests that recovery of upper limb strength is also important during the outpatient rehabilitation period and beyond. The insights gained in the longitudinal relationship of improvement in HRQoL in relation to improvement in arm recovery can be useful for the understanding of problems faced by patients, and for planning and optimization of rehabilitation treatment after stroke (Chapter 4).

An exploratory analysis revealed that self-identified goals of chronic stroke patients with upper extremity dysfunction were more activity-focused than impairment-focused. ${ }^{27}$ The goals identified were mainly activities that require the use of both hands, regardless of whether the right- or left upper limb was affected after stroke. Rehabilitation in the late subacute to chronic phase after stroke could therefore benefit from focusing on activitybased rather than on impairment-based upper limb interventions. Collectively, our results and those of others are in line with the definition of effective rehabilitation, which is a person-centered process, and where treatment is tailored to a patient's unique set of needs and goals. ${ }^{28,29}$ Within in- and outpatient stroke rehabilitation, there must be a permanent focus on the goal-setting process, resulting in an individualized set of goals derived from the patient's perspective.

\section{Aiming for optimal recovery - NIBS in the rehabilitation of stroke}

\section{Treatment timing}

Most studies and RCTs on neurorehabilitation have been conducted in patients with chronic stroke whereas most conventional neurorehabilitation is provided in the first three months after stroke. ${ }^{30}$ The early post-stroke period is important, since the largest amount of upper extremity recovery occurs in the first 4 weeks and reaches a plateau at approximately 3 months post-stroke. ${ }^{31-33}$ However, the most optimal timing, duration, and intensity of early rehabilitation interventions remain to be determined. ${ }^{34}$ In Chapter 5, we reviewed literature and performed a meta-analysis to identify differences in the efficacy of rTMS treatment on upper limb function depending on the time of start of treatment after stroke. 
We found that rTMS within 1 month after stroke has lead to greater improvement on the FM-UE than rTMS applied after 1-3 months or after 6 months. This beneficial effect of early rTMS has not previously been reported, since previous meta-analyses did not take into account the different time intervals between stroke onset, treatment initiation and outcome measurements, and pooled the different outcome measures. Furthermore, no previous systematic review and meta-analysis has examined the impact of the timing after stroke when a rehabilitation intervention is delivered.

Most phase II trials of rTMS included patients at arbitrary time-points relative to the onset of stroke, which introduces large heterogeneity between studies. This heterogeneity creates additional variance, which results in a larger number of patients needed to achieve sufficient statistical power. ${ }^{35}$ Current upper limb rehabilitation interventions do not appear to have an impact on recovery rates beyond what can be expected from spontaneous biological recovery. ${ }^{36,37}$ Recovery in the early post-stroke period is often referred to as spontaneous biological recovery as it is attributable to endogenous repair processes, rather than driven by current rehabilitation approaches. ${ }^{36}$ However, cellular and physiological conditions that allow spontaneous recovery may also increase responsiveness to (intense) training. ${ }^{37}$ The effect of early started rTMS (Chapter 5) is consistent with theories about a possible interaction between interventions and spontaneous neurobiological mechanisms. ${ }^{35,38,39}$ Reasons why very few studies in humans have explicitly assessed the benefits of (intense) training in the early post-stroke period include logistics, economics, and evidence from rodent studies that very early intense therapy (e.g. within 24 hours) may lead to increased cell death and exacerbate injury. ${ }^{36,40,41}$ The few early initiated trials in humans did not have an impairmenttargeted treatment (EXPLICIT; EXplaining PLastICITy), ${ }^{42}$ or impairment was not measured at all (VECTORS; Very Early Constraint-Induced Movement during Stroke Rehabilitation, AVERT; A Very Early Rehabilitation Trial, and ICARE; Interdisciplinary Comprehensive Arm Rehabilitation Evaluation). ${ }^{33,43,44}$ Furthermore, none of the studies have directly compared the effects of starting treatment early after stroke to starting at a later time-point. Therefore, the results from these early trials that intervening too early post-stroke might be maladaptive need to be interpreted with caution.

\section{Outcome measurement}

As was shown in Chapter 5, rTMS seems to have a positive effect on upper limb function if assessed with tests that target (body) function specifically, which was not evident with tests assessing activity. Stroke-related brain changes are best assessed with impairment measures as compared to outcome measures at the level of activity, because improvements on functional outcome measures are contaminated by compensation. Improvement on a functional outcome measure may occur through compensation without any restitution. 
Around 1980 there was a shift from treatment of impairment toward training on activities of daily living with functional tasks, because of the realization that motor training and task practice will generalize to ADLs, as teaching normal movement patterns did not. ${ }^{37,45}$ This shift also led to a move toward global disability scales in clinical practice, and task-specific functional measures in research studies. In 2017, the Stroke Recovery and Rehabilitation Roundtable responded to the lack of standardized approaches for measurements in stroke recovery research by achieving consensus about a core set of valid measures that should be used in every stroke recovery and rehabilitation trial and be assessed at fixed times post stroke. ${ }^{13}$

\section{Treatment dosage}

In addition to the association between time to start therapy after stroke and outcome, the dose of therapy is another important factor within rehabilitation interventions. The traditional forced bedrest after stroke has been replaced by the hypothesis of mobilizing as early as possible to promote recovery. ${ }^{46}$ The intensity and dose of current rehabilitation treatments is probably not sufficient to exceed threshold levels for improving true neurological recovery. ${ }^{39}$ Systematic reviews have pointed out that stroke survivors have substantial periods of inactivity. During inpatient rehabilitation, activity of the affected upper limb was on average 4 minutes per session during physiotherapy, and 17 minutes per session during occupational therapy. ${ }^{47}$ Within the neuromodulation (rTMS) field, the intensity of the treatment is mainly determined by the number of sessions. As shown in Chapter 5, there were significant beneficial effects on ICF body function and structure measures for varying amount of treatment sessions (i.e., single treatment session, 2-10 or 11-20 sessions), however this finding is based on a few studies within the different phases of treatment onset post-stroke. The number of sessions, and with this the intensity of the treatment, have not yet received much attention within stroke rehabilitation.

\section{Trial optimization}

In Chapter 6, the study protocol of the RCT B-STARS was described. Key elements of B-STARS are start of rTMS treatment within 3 weeks after stroke onset, outcome assessments serially at fixed time-points after stroke, stratification at baseline based on finger extension, and follow-up until one-year post-stroke. These key elements are in line with recommendations from studies on upper limb recovery trial design. ${ }^{13,35}$ We chose to use continuous TBS (cTBS) of the contralesional primary motor cortex, because evidence on efficacy and longterm effects on arm function of contralesional TBS in patients with subacute hemiparetic stroke is still lacking (Chapter 6). The interhemispheric competition model (Chapter 1) has been the basis for our stimulation protocol. In more than 50 small clinical trials, identified 
out of the 350 articles that have been published on NIBS in stroke in the last five years, modest evidence was found for inhibitory or excitatory stimulation, which questions the applicability of the interhemispheric competition model to all patients with stroke. ${ }^{48}$ The rise of alternative models for stimulation protocols, such as the bimodal balance recovery model, suggests that there is a need to modify one-size-fit-all stimulation protocols. In this thesis, we have not yet examined the effects of cTBS on upper limb function for patients included in B-STARS since the data collection is still in progress.

\section{Patient participation}

In the qualitative interviews we did with patients who participated in B-STARS (Chapter 7), we revealed that rTMS was well accepted and even enjoyed by the patients. Comfortable treatment settings, respectful communication, and transparent information contributed to the experienced satisfaction. Surprisingly, participation in the trial was also felt as a gratifying experience and gave patients a sense of purpose. Outcome measures of functioning and disability, including the PROMs, may not capture the personal experiences of patients who participate in a clinical trial or other research study. It is also of great value to gather the experiences and opinions of patients who participated in clinical research, since these insights may help researchers and healthcare professionals to identify topics that are important for patients undergoing a treatment, which could improve future trial design and subsequent clinical implementation. Expectations can also play an important role in the outcome of a treatment. ${ }^{49}$ As such, qualitative research has the potential to improve rehabilitation, since we can explore the benefit or harm of an intervention from the patient's perspective.

Evidence-Based Medicine (EBM) dominates the field of healthcare research, with emphasis on objectivity and hypothesis testing, which complicates the acceptance and use of qualitative research. Multiple factors, such as paradigm shifts in the philosophy of science, increased popularity of mixed-methods approaches and advances in methodology in qualitative research, have nevertheless led to widening interest in the use of qualitative research. ${ }^{50}$ Several studies suggest that detailed understanding of clinical outcomes and concerns, such as practitioner-patient interaction, is best addressed by including the patient's perspective instead of using quantitative methods alone. ${ }^{51,52}$ The recognition of the limitations of monomethod studies, along with the release of best practices for mixed methods design and an increase in federally funded mixed methods research proposals, have led to the rise of mixed method research in health sciences. ${ }^{53,54}$ Qualitative data, in the form of stories or narratives, also has the potential to facilitate knowledge translation through the ease of comprehension, especially to non-academic audiences (e.g. general public). ${ }^{55}$ According to a recent paper based on literature review and the authors' collective experiences, advanced training in qualitative methods and engaging qualitative communities of research (i.e. group of people 
that share experiences and learning about research and practice), are needed to advance the future possibilities and potential of qualitative research in rehabilitation science. ${ }^{50,56}$

\section{Treatment implementation}

It has been estimated that there is an average time lag of 17 years of getting research evidence into practice. ${ }^{57}$ There are several reasons for implementation delays. An intervention might be ineffective in a new setting or a good intervention could be implemented incorrectly. Distinguishing treatment effectiveness from implementation effectiveness could lead to more rapid translational gains. While quantitative approaches can assess outcomes of an intervention, qualitative studies can also serve as a tool to identify barriers and facilitators to implement evidence-based practice or to identify strategies for facilitating implementation. ${ }^{58,59}$ In Chapter 7, we addressed the relevance of the endusers (i.e. stroke patients) of the rTMS treatment (i.e. implementation object), which may influence implementation effectiveness, however a systems approach to implementation is needed. Many so-called determinant frameworks acknowledge that implementation is a multidimensional phenomenon, and they point to multiple influences at different levels, from the individual user or adopter (e.g. health care professional) to the context and beyond. ${ }^{60}$ I believe that blending design components of clinical effectiveness and implementation research within a study could speed up getting practices implemented in the real world.

\section{Methodological considerations}

\section{Study population and design}

For this thesis, data was obtained from several studies and from the existing literature. In the B-STARS study, we recruited patients at admission to the rehabilitation center, thereby focusing solely on the inpatient stroke rehabilitation population, which is about $10-15 \%$ of the total stroke population. Randomization was done within the first 3 weeks after stroke onset. The inclusion of both ischemic and hemorrhagic strokes will result in reduced homogeneity between the patients. However, we used prognostic stratification, based on voluntary finger extension, contributing to homogeneity between patients and the prevention of type-II errors..$^{61,62}$

The EXPLICIT study also included patients in the subacute phase after stroke. Randomization was also done within the first 3 weeks after stroke onset, and prognostic stratification was used based on voluntary finger extension. The EXPLICIT study included only patients with a first-ever ischemic stroke. The restricted inclusion criteria in the EXPLICIT study led to a slow recruitment rate, and a smaller proportion of patients were included than initially planned. 
On the contrary, the FIT-Stroke study targeted the outpatient stroke rehabilitation population; patients with stroke discharged from a rehabilitation center to their own home. In this study, the inclusion criteria were based on being able to walk a minimum of $10 \mathrm{~m}$ without physical assistance from a therapist, and the need to continue physiotherapy during outpatient care to improve walking competency or physical condition. Despite the specific selection for inclusion in a task-oriented circuit class training, only a few patients reached a maximum score on the upper limb clinical measures at baseline. Only a small group of mild to moderate stroke patients could be selected, since two-third of the patients showed no or insufficient walking problems, or were too ill to be included, which limits the generalizability.

The longitudinal design of the RCTs resulted in follow-up assessments up to 1-year poststroke. In all studies, we included a three-month assessment post-stroke. Most rehabilitative intervention RCTs take place in the chronic phase after stroke, and do not include assessments at fixed time-points. Therefore, in this thesis we provided new information to the existing literature on the early-, late-, and chronic time-points post-stroke, by using standard time-points aligned with underlying recovery processes. We feel that B-STARS, EXPLICIT, and FIT-Stroke complement each other well because of differences in study population and design, and therefore provide a broad overview of the stroke patient rehabilitation in the Netherlands.

We could have involved patient representatives and their input on the design of the B-STARS trial at an earlier stage (i.e. during the set-up of the trial) instead of halfway through the trial. Considering that patients may have different needs regarding information, communication and involvement within a clinical trial, involving a patient's representative could contribute to a better match between the design of the trial and the wishes of a stroke patient. The move toward patient-centered medicine is not happening at the same rate throughout clinical research, however there are increasingly more examples and recommendations of how patients can achieve the level of true partnership in the research process. ${ }^{63}$

\section{Outcome measures}

In our studies on post-stroke motor outcome we did not assess somatosensory function. Research indicates that upper limb motor performance, pinch grip deficits, and participation outcomes are related to somatosensory impairments after stroke. ${ }^{64,65}$ The exploration of somatosensory loss could have told us more about the underlying processes (specifically the impact of somatosensory impairment) involved in motor recovery at different time-points post-stroke. Unfortunately, due to a different focus of our study and logistic reasons, we could not include sensorimotor assessments. The assessment of more psychological factors (i.e. helplessness and passive coping) as part of the outcome measurements would have 
been relevant, since previous research revealed that psychological factors are important determinants for HRQoL, in comparison to demographic and stroke-related factors that only explain a small part of the variance in HRQoL. ${ }^{66}$ When we would have taken these factors into account, we would have created a more complete picture of determinants of HRQOL and motor recovery in stroke patients.

\section{Clinical implications}

Based on the findings from this thesis as well as from clinical experience, we formulated clinical recommendations to improve the assessment of upper limb recovery and the use of non-invasive brain stimulation after stroke.

Develop a core, standardized set of measurements at fixed time-points post-stroke - at the function, activity and participation levels of ICF

We believe that the primary outcome measure should reflect the underlying rationale or mechanism of the intervention under study. In addition, the inclusion of a core, standardized set of (secondary) measurements at the different levels of function and activity, will move stroke rehabilitation forward. Furthermore, the reporting of findings from trials should be done in a uniform manner (e.g., using final scores or change scores, and subtest scores). The identification of 208 unique upper limb function assessment tools affirms the lack of a standardized approach for measurements in stroke recovery research. ${ }^{67}$ As we have shown in Chapter 5, the great variety in outcome measures and reporting of findings in clinical trials (i.e. final scores, change scores) hinder the comparability across different trials in meta-analyses. Furthermore, pooling of studies with outcome measures at different levels of ICF (i.e. function and activity) lowers the likelihood of finding specific treatment effects.

Use outcome measures that differentiate between true recovery and compensation An often-overlooked factor in behavioral recovery is the difference between true recovery and compensation. In translating preclinical findings to the clinic, it is also often neglected that true recovery and compensation are different. To improve our understanding of neurobiological recovery patterns, we should include measures as the skilled reaching task, that distinguish between true recovery and compensation. As we have shown in Chapter 2 , there is a strong relation between human skilled reaching and commonly used clinical outcome measures. The insights gained from Chapter 2 may also be of interest for the debate on the utility and relevance of rodent models for translational stroke recovery research. 


\section{Add PROMs to a core, standardized set of measurements}

With regard to clinical outcome measures, self-reported measures can provide additional insights into the impact of disability on the patient, beyond what is provided by observational measures alone (Chapter 3). Self-reported measures can help to design optimal rehabilitation strategies for patients who underestimate their capacity (e.g., training in the use of the affected hand, positive psychology, self-efficacy, and expectation management). For the patients who overestimate their capacity, training in body image may be provided. Patients also value feedback on their progress of motor recovery, as it contributes to an individual's expectation, motivation, and attempts to maximize upper limb recovery (Chapter 7). Therefore, repeated measurements at fixed time-points after stroke onset should be implemented in clinical rehabilitation settings in order to inform patients about their recovery and set treatment goals. Furthermore, repeated measurements will also enable the collection of recovery profile data to enhance the development and validation of prediction models.

Offer and make therapy and monitoring for arm recovery also available after three months post-stroke

Our results imply that there should be monitoring of recovery and treatment of upper limb strength during the outpatient rehabilitation period and beyond, i.e. outside the typical time window of recovery in the first 3 months post-stroke, as HRQoL is associated with improvement in upper limb strength recovery in this time window (Chapter 4). Despite the majority of research studies focusing on chronic stroke patients, in clinical practice the therapy for these patients is close to nonexistent and they rarely receive significant amounts of therapy. Improving functional independence of stroke patients is a primary goal of clinical practice. Greater efforts are needed to ensure that improvement of functional independence is also a primary goal outside the therapeutic environment. The choice of treatment should be determined based on evidence from future studies in this field.

Implement rTMS treatment in stroke rehabilitation - if proven effective Our findings from the interviewed patients revealed that rTMS within an inpatient rehabilitation setting was well accepted and even enjoyed by patients, and was not experienced as a burden (Chapter 7).

\section{Add short moments of relaxation to stroke inpatient rehabilitation programs}

The moment of relaxation while receiving the rTMS, which contributed to the positive experiences of the patients, also has a clinical implication. The moment of relaxation in- 
between appointments of a rehabilitation program, with the freedom to do nothing, could be built into the standard routine of inpatient stroke rehabilitation.

\section{Recommendations for future research}

In recent years it has become clear that further research and international cooperation should be undertaken to develop a standardized, core set of measurements for testing upper limb function. We recommend to conduct measurements at the different levels of function, activity, and participation. Patient-centered outcomes should also be included in this core set. In addition, future clinical trials on upper limb recovery would benefit from measuring somatosensory deficits, in addition to the functional arm outcomes, to evaluate functional gains from sensorimotor interventions and guide realistic treatment goals. Future studies should incorporate standardized tests, include at least a follow-up measurement at three months after stroke onset, especially when the trial starts within one-month poststroke, and report their findings in a uniform manner (e.g., using final scores or change scores, and subtest scores).

To progress towards individualized therapy, further research in the field of NIBS will have to be conducted in order to develop and validate biomarkers associated with a response to NIBS (e.g. neuroimaging-based connectivity measures), since there is inevitable heterogeneity between and within stroke subjects. Advanced induced electrical field modeling could aid in refining targeting by predicting the changes induced by NIBS on a sub-regional level.68,69 Development of closed-loop approaches could lead to incorporation of activity-dependent TMS in stroke rehabilitation, which holds promise for more efficacious recovery-promoting plasticity induction. ${ }^{70}$ New non-invasive brain stimulation approaches, such as transcranial focused ultrasound, ${ }^{71}$ which can target deep brain structures inaccessible by TMS and tDCS, can also help to move the field forward. However, the lack of cohesion in the current literature should be targeted first by standardized measurements, reporting, and baseline stratification in research.

Finally, we believe that the use of mixed methods designs, i.e. where qualitative studies are carried out alongside randomized controlled trials, can advance the field of stroke rehabilitation. This will improve the understanding of the effects of interventions and how they are experienced by recipients as well as healthcare professionals. Further efforts are needed to develop and advance methodological guidance for qualitative researchers to ensure the needed expertise to conduct high-quality research. Qualitative research can also aid in establishing the barriers and facilitators of implementation of stroke research findings into clinical practice. Collaboration with implementation scientists could be of great help in the design of implementation studies and measuring implementation outcomes. In 
combination with the previously mentioned recommendations, this could further facilitate the translation of advancements in stroke rehabilitation research into clinical practice.

"The future of stroke recovery requires that we give humans wings." ${ }^{37}$ (p.222) Proverbial wings, so that we take the right steps from a bird's-eye view to further develop the stroke recovery field. The wings can also be seen as the windings of a TMS coil, which can improve the stroke recovery field in both diagnostics and treatment.

\section{References}

1. Alaverdashvili, M. \& Whishaw, I. Q. A behavioral method for identifying recovery and compensation: Hand use in a preclinical stroke model using the single pellet reaching task. Neurosci. Biobehav. Rev. 37, 950-967 (2013).

2. Klein, A., Sacrey, L. A. R., Whishaw, I. Q. \& Dunnett, S. B. The use of rodent skilled reaching as a translational model for investigating brain damage and disease. Neurosci. Biobehav. Rev. 36, 1030-1042 (2012).

3. Kleim, J. A., Boychuk, J. A. \& Adkins, D. A. L. Rat models of upper extremity impairment in stroke. ILAR Journal 48, 374-384 (2007).

4. Sena, E., van der Worp, H. B., Howells, D. \& Macleod, M. How can we improve the pre-clinical development of drugs for stroke? Trends Neurosci. 30, 433-439 (2007).

5. Winters, C. et al. Moving stroke rehabilitation forward: The need to change research. NeuroRehabilitation 43, 19-30 (2018).

6. Levin, M. F., Kleim, J. A. \& Wolf, S. L. What Do Motor Recovery and Compensation Mean in Patients Following Stroke? Neurorehabil. Neural Repair 23, 313-319 (2008).

7. Jones, T. Motor compensation and its effects on neural reorganization after stroke. Nat. Rev. Neurosci. 18, 276-278 (2017).

8. Sacrey, L. A. R., Alaverdashvili, M. \& Whishaw, I. Q. Similar hand shaping in reaching-for-food (skilled reaching) in rats and humans provides evidence of homology in release, collection, and manipulation movements. Behav. Brain Res. 204, 153-161 (2009).

9. Whishaw, I. Q., Pellis, S. M. \& Gorny, B. P. Skilled reaching in rats and humans: evidence for parallel development or homology. Behav. Brain Res. 47, 59-70 (1992).

10. Bernhardt, J. et al. Moving rehabilitation research forward: Developing consensus statements for rehabilitation and recovery research. Int. J. Stroke 11, 454-458 (2016).

11. Corbett, D. et al. Enhancing the alignment of the preclinical and clinical stroke recovery research pipeline: Consensus-based core recommendations from the Stroke Recovery and Rehabilitation Roundtable translational working group. Int. J. Stroke 12, 462-471 (2017).

12. Balkaya, M. \& Cho, S. Optimizing functional outcome endpoints for stroke recovery studies. J. Cereb. Blood Flow Metab. 39, 2323-2342 (2019).

13. Kwakkel, G. Standardised measurement of sensorimotor recovery in stroke trials: consensus-based core recommendations from the Stroke Recovery and Rehabilitation Roundtable (SRRR). Int. J. Stroke 12, 451-461 (2017).

14. Reeves, M. et al. Patient-Reported Outcome Measures (PROMs) for Acute Stroke: Rationale, Methods and Future Directions. Stroke 49, 1549-1556 (2018).

15. Groeneveld, I. et al. Value-Based Stroke Rehabilitation: Feasibility and Results of Patient-Reported Outcome Measures in the First Year After Stroke. J. Stroke Cerebrovasc. Dis. 28, 499-512 (2019). 
16. Rumsfeld, J. S. et al. Cardiovascular health: The importance of measuring patient-reported health status a scientific statement from the American heart association. Circulation 127, 2233-2249 (2013).

17. Salinas, J. et al. An international standard set of patient-centered outcome measures after stroke. Stroke 47, 180-186 (2016).

18. Hartman-Maier, A., Soroker, N., Ring, H. \& Katz, N. Awareness of deficits in stroke rehabilitation. J. Rehabil. Med. 34, 158-164 (2002).

19. Toglia, J. \& Kirk, U. Understanding awareness deficits following brain injury. NeuroRehabilitation 15, 57-70 (2000).

20. van Delden, A. (Lex) E. Q., Peper, C. (Lieke) E., Beek, P. J. \& Kwakkel, G. Match and mismatch between objective and subjective improvements in upper limb function after stroke. Disabil. Rehabil. 35, 1961-1967 (2013)

21. Hochstenbach, J., Prigatano, G. \& Mulder, T. Patients' and relatives' reports of disturbances 9 months after stroke: Subjective changes in physical functioning, cognition, emotion, and behavior. Arch. Phys. Med. Rehabil. 86, 1587-1593 (2005).

22. Persson, H. C., Danielsson, A. \& Sunnerhagen, K. S. A cross sectional study of upper extremity strength ten days after a stroke; relationship between patient-reported and objective measures. BMC Neurol. 15, 1-7 (2015).

23. Essers, B. et al. Mismatch between observed and perceived upper limb function: an eye-catching phenomenon after stroke. Disabil. Rehabil. 41, 1545-1551 (2019).

24. Barrett, A. M. Rose-colored answers: Neuropsychological deficits and patient-reported outcomes after stroke. Behav. Neurol. 22, 17-23 (2009).

25. Boyce, M. B., Browne, J. P. \& Greenhalgh, J. The experiences of professionals with using information from patient-reported outcome measures to improve the quality of healthcare: A systematic review of qualitative research. BMJ Qual. Saf. 23, 508-518 (2014).

26. Meehan, T., Mccombes, S., Hatzipetrou, L. \& Catchpoole, R. Introduction of routine outcome measures: Staff reactions and issues for consideration. J. Psychiatr. Ment. Health Nurs. 13, 581-587 (2006)

27. Waddell, K. J., Birkenmeier, R. L., Bland, M. D. \& Lang, C. E. An exploratory analysis of the selfreported goals of individuals with chronic upper-extremity paresis following stroke. Disabil. Rehabil. 38, 853-857 (2016).

28. Wade, D. What is rehabilitation? An empirical investigation leading to an evidence-based description. Clin. Rehabil. 34, 571-583 (2020).

29. Waddell, K. J., Birkenmeier, R. L., Bland, M. D. \& Lang, C. E. An exploratory analysis of the selfreported goals of individuals with chronic upper-extremity paresis following stroke. Disabil. Rehabil. 38, 853-857 (2016).

30. Stinear, C., Ackerley, S. \& Byblow, W. Rehabilitation is initiated early after stroke, but most motor rehabilitation trials are not: A systematic review. Stroke 19, 59 (2013).

31. Duncan, P. W., Goldstein, L. B., Matchar, D., Divine, G. W. \& Feussner, J. Measurement of motor recovery after stroke. Stroke 23, 1084-1089 (1992).

32. Bernhardt, J. et al. Agreed definitions and a shared vision for new standards in stroke recovery research : The Stroke Recovery and Rehabilitation Roundtable taskforce. Int. J. Stroke 12, 444-450 (2017).

33. Dromerick, A. W. et al. Very early constraint-induced movement during stroke rehabilitation (VECTORS): A single-center RCT. Neurology 73, 195-201 (2009).

34. Kwakkel, G., Veerbeek, J. M., Wegen, E. Van \& Wolf, S. L. Constraint induced movement therapy after stroke. HHS Public Access 14, 224-234 (2016). 
35. Winters, C., Heymans, M. W., van Wegen, E. E. H. \& Kwakkel, G. How to design clinical rehabilitation trials for the upper paretic limb early post stroke? Trials 17, 1-9 (2016).

36. Zeiler, S. R. Should We Care About Early Post-Stroke Rehabilitation? Not Yet, but Soon. Curr. Neurol. Neurosci. Rep. 19, 13 (2019).

37. Krakauer, J. W. \& Carmichael, S. T. Broken Movement. Broken Movement (2019). doi:10.7551/ mitpress/9310.001.0001

38. Biernaskie, J. Efficacy of rehabilitative experience declines with time after focal ischemic brain injury. J. Neurosci. 24, 1245-1254 (2004).

39. Krakauer, J. W., Carmichael, S. T., Corbett, D. \& Wittenberg, G. F. Getting neurorehabilitation right: what can be learned from animal models? Neurorehabil. Neural Repair 26, 923-931 (2012).

40. Shen, J. et al. Early rehabilitation aggravates brain damage after stroke via enhanced activation of nicotinamide adenine dinucleotide phosphate oxidase (NOX). Brain Res. 1648, 266-276 (2016).

41. Kozlowski, D. A., James, D. C. \& Schallert, T. Use-dependent exaggeration of neuronal injury after unilateral sensorimotor cortex lesions. J. Neurosci. 16, 4776-4786 (1996).

42. Kwakkel, G. et al. Effects of unilateral upper limb training in two distinct prognostic groups early after stroke. Neurorehabil. Neural Repair 30, 804-816 (2016).

43. Bernhardt, J. et al. Efficacy and safety of very early mobilisation within $24 \mathrm{~h}$ of stroke onset (AVERT): A randomised controlled trial. Lancet 386, 46-55 (2015).

44. Winstein, V. J. et al. Effect of a Task-Oriented Rehabilitation Program on Upper Extremity Recovery Following Motor Stroke. HHS Public Access 315, 571-581 (2016).

45. Carr, J. H. Movement science: Foundations for physical therapy in rehabilitation. (Aspen Publishers, 1987).

46. Coleman, E. R. et al. Early Rehabilitation After Stroke: a Narrative Review. Curr. Atheroscler. Rep. 19, 59 (2017).

47. Hayward, K. S. \& Brauer, S. G. Dose of arm activity training during acute and subacute rehabilitation post stroke: A systematic review of the literature. Clin. Rehabil. 29, 1234-1243 (2015).

48. Di Pino, G. et al. Modulation of brain plasticity in stroke: a novel model for neurorehabilitation. Nat. Rev. Neurol. 10, 597-608 (2014).

49. Evers, A. W. M. et al. Implications of placebo and nocebo effects for clinical practice: Expert consensus. Psychother. Psychosom. 87, 204-210 (2018).

50. VanderKaay, S. et al. Qualitative research in rehabilitation science: opportunities, challenges, and future directions. Disabil. Rehabil. 40, 705-713 (2018).

51. Greenhalgh, T. et al. An open letter to the BMJ editors on qualitative research. BMJ 352, i563 (2016).

52. Morse, J. M. Qualitative health research : one quarter of a century. Qual. Health Res. 25, 3-4 (2015).

53. Coyle, C. E. et al. Federal Funding for Mixed Methods Research in the Health Sciences in the United States: Recent Trends. J. Mix. Methods Res. 12, 270-283 (2018).

54. Creswell, J., Klassen, A., Plano Clark, V. \& Smith, K. Best practices for mixed methods research in the health sciences. National Institutes of Health Office of Behavioral and Social Sciences Research (2018).

55. Cunningham, R. M. \& Boom, J. A. Telling stories of vaccine-preventable diseases: why it works. S. D. Med. 21-6 (2013).

56. Cheek, J., Corlis, M. \& Radoslovich, H. Connecting what we do with what we know: building a community of research and practice. Int. J. Older People Nurs. 4, 233-238 (2009).

57. Morris, Z. S., Wooding, S. \& Grant, J. The answer is 17 years. J. R. Soc. Med. 104, 510-520 (2011).

58. Palinkas, L. A. et al. Mixed method designs in implementation research. Adm. Policy Ment. Heal. Ment. Heal. Serv. Res. 38, 44-53 (2011).

59. Powell, B. J. et al. Innovations in Health and Mental Health. Med. care Res. Rev. 69, 1-28 (2013). 
60. Nilsen, P. Making sense of implementation theories, models and frameworks. Implement. Sci. 10, 1-13 (2015).

61. Langhorne, P., Bernhardt, J. \& Kwakkel, G. Stroke rehabilitation. Lancet 377, 1693-1702 (2011).

62. Veerbeek, J. M. et al. What is the evidence for physical therapy poststroke? A systematic review and meta-analysis. PLoS One 9, (2014).

63. Sacristán, J. A. et al. Patient involvement in clinical research: Why, when, and how. Patient Prefer. Adherence 10, 631-640 (2016).

64. Meyer, S. et al. Associations between sensorimotor impairments in the upper limb at 1 week and 6 months after stroke. J. Neurol. Phys. Ther. 40, 186-195 (2016).

65. Carey, L. M., Matyas, T. \& Baum, C. Effects of Somatosensory Impairment on Participation After Stroke. Am. J. Occup. Ther. 72, 7203205100p1-7203205100p10 (2018).

66. van Mierlo, M., van Heugten, C., Post, M. W. M., Hoekstra, T. \& Visser-Meily, A. Trajectories of health-related quality of life after stroke: results from a one-year prospective cohort study. Disabil. Rehabil. 40, 997-1006 (2018).

67. Pollock, A. et al. Cochrane overview: Interventions for improving upper limb function after stroke. Stroke 46, e57-e58 (2015).

68. Salo, K. S. T., Vaalto, S. M. I., Mutanen, T. P., Stenroos, M. \& Ilmoniemi, R. J. Individual Activation Patterns after the Stimulation of Different Motor Areas: A Transcranial Magnetic StimulationElectroencephalography Study. Brain Connect. 8, 420-428 (2018).

69. Ovadia-Caro, S. et al. Predicting the response to noninvasive brain stimulation in stroke. Front. Neurol. 10, 302 (2019).

70. Gharabaghi, A. et al. Coupling brain-machine interfaces with cortical stimulation for brain-state dependent stimulation: Enhancing motor cortex excitability for neurorehabilitation. Front. Hum. Neurosci. 8, 122 (2014).

71. Fini, M. \& Tyler, W. J. Transcranial focused ultrasound: a new tool for non-invasive neuromodulation. Int. Rev. Psychiatry 29, 168-177 (2017) 


\section{Summary \\ Samenvatting}





\section{Summary}

Each year more than 15 million people worldwide have a stroke, which is a major cause of adult disability. The impact of this common and serious condition on an individual's life is considerable: physical, psychological, and social consequences can be experienced. Upper limb paresis (muscle weakness) is one of the most frequently occurring conditions (in up to $85 \%$ of stroke survivors). Functional impairments can lead to disability and an inability or difficulty in performing everyday activities of daily living, such as dressing. Whilst some research has been carried out on the association between arm function and health-related quality of life (HRQoL), there has been no previous longitudinal investigation of changes in $\mathrm{HRQ} O \mathrm{~L}$ in relation to improvement in arm recovery.

Stroke recovery is different for each patient and occurs at a varying pace. Recovery is usually most rapid in the first six months after a stroke. At 6 months post-stroke, some dexterity in the paretic arm can be found in more than one-third of the stroke patients who showed no dexterity in the first week post-stroke. It has been strongly recommended to actively perform rehabilitation from an early stage after stroke in order to enhance the recovery of neurologic sequelae. Arm function plays a critical role in the performance of daily life activities. Many everyday activities require the use of both hands, and because of this, performance of bimanual activities receives considerable attention in the rehabilitation setting.

Since there are many rehabilitative interventions available, often a therapist's clinical reasoning determines the selection of (evidence-based) therapy in line with the goals of the patient. Most studies on neurorehabilitation have been conducted in patients with chronic stroke (> 6 months after stroke onset), while the first three-months period after stroke is often referred to as the critical time-window for increased brain plasticity (the brain's ability to change and adapt as a result of experience). Unfortunately, evidence in favor of early utilization of plasticity-inducing interventions for stroke rehabilitation is still lacking. There has been a particularly growing interest in the use of non-invasive neuromodulation to enhance motor function recovery after stroke. However, most brain stimulation studies were conducted in chronic stroke patients, and very limited follow-up data on the longterm effects of repetitive transcranial magnetic stimulation (rTMS) intervention for stroke rehabilitation are available.

This thesis aimed to provide a perspective on the assessments of upper limb recovery used after stroke during rehabilitation, and the potential of non-invasive brain stimulation techniques, particularly TMS, to improve upper limb performance.

Chapter 1 provides an overview of the context of this Thesis. The consequences of stroke on upper limb function and health-related quality of life are discussed. TMS is introduced as 
a neuromodulatory approach to enhance true recovery, and the monitoring and evaluation of motor recovery is emphasized.

\section{Part I Understanding and assessment of post-stroke impairment and recovery}

In Chapter 2 the similarities between human and rodent reaching movements of the arm and hand (skilled reaching; conventional term for reach-to-eat) in the sub-acute phase after stroke are evaluated, as well as the relation between skilled reaching and clinical outcome measures. Testing of skilled reaching (or single pellet reaching) provides a behavioural assessment that allows for distinction between true recovery and compensation, and it can be executed in rodents as well as in humans. This generalizability enhances the expectation that principles derived from preclinical assessment of the behavior are clinically applicable. A total of twelve human subjects and seventeen rats, all with a stroke, were included from the B-STARS trial (Brain STimulation for Arm Recovery after Stroke) and a similar designed preclinical study, respectively. Skilled reaching movement patterns showed significant homologies between rodents and humans. Over time, patients and rats improved significantly on movement elements of the skilled reaching task. Both species showed muscle flaccidity at the first measurement post-stroke and a delayed recovery in distal muscles of the lower arm and hand/paw. Some movements elements in rats (i.e. digits semi-flexed, aim, pronation, and grasp) showed no improvement over time, as these movements were close to normal subacutely after stroke. Skilled reaching showed strong correlations with well-used clinical outcome measures in the first three months post-stroke. In conclusion, skilled reaching testing offers an effective and highly translational means for assessment of motor recovery in experimental and clinical stroke settings.

Chapter 3 focuses on the comparison of self-reported versus observational clinical measures of improvement in upper limb capacity in patients after stroke. Recovery of the paretic arm can be assessed by a clinician (observational) or by the patient (patient-reported). A total of 159 patients with ischemic stroke with upper limb paresis were included in the study. The timeframe post-stroke ( $0-3$ or 3-6 months) did not seem to influence the correspondence between the observational and self-reported measures: there were more matches than mismatches found between the measures. In case of mismatches, patients' self-reports were more often pessimistic (86\%) in the first 3 months post-stroke compared to the subsequent 3 months (39\%). Self-reported measures can be used in addition to observational measures to assess arm recovery. Information on the ability and use of the affected arm outside the treatment setting is valuable for clinicians, as it provides more insight into the patients' perspective.

In Chapter 4, we investigated to what extent changes in $\mathrm{HRQOL}$ are related to changes in upper limb strength after discharge from inpatient rehabilitation. A total of 250 patients 
from the FIT-Stroke trial were assessed at discharge from inpatient rehabilitation (baseline) and at 12 weeks post-discharge (follow-up). We found that upper limb strength at discharge from an inpatient rehabilitation setting was a major predictor of HRQoL three months later, after accounting for demographic and clinical characteristics. Improvement in HRQoL was positively related to improvement in upper limb strength. Better monitoring of recovery and treatment of upper limb strength during the outpatient rehabilitation period and beyond, i.e. outside the typical time-window of recovery in the first 3 months post-stroke, might contribute to higher quality of life for stroke survivors.

Part II Aiming for optimal recovery - non-invasive brain stimulation in the rehabilitation of stroke

A systematic review and meta-analysis on the timing of rTMS onset for upper limb function after stroke is described in Chapter 5. A total of 38 studies involving 1,074 stroke patients met the inclusion criteria and were included. Timing of treatment initiation post-stroke was categorized as follows: acute to early subacute (< 1 month), early subacute (1-3 months), late subacute (3-6 months), and chronic (> 6 months). rTMS was beneficial when it was applied within the first month post-stroke, but not in the early sub-acute phase or chronic phase post-stroke. This beneficial effect was only found for outcome measures at the level of function (e.g. Fugl-Meyer arm score), but not for outcome measures at the level of activity. However, rTMS treatment studies in stroke patients were highly heterogeneous, with varying outcome measures and relatively small sample sizes. Therefore, the results need to be interpreted with caution. Based on the findings of this study, the recommendation was done to develop a standardized, core set of measurements for testing upper limb function.

Chapter 6 describes the study protocol of the clinical trial B-STARS. The primary objective of this study, which was still ongoing during the writing of this thesis, is to determine the therapeutic effect of contralesional cTBS (inhibitory stimulation) on recovery of function of the paretic arm at 3 months after stroke. B-STARS is a prospective, randomized, double-blind, controlled clinical trial. Subjects are randomly allocated to real or sham brain stimulation, followed by standard care upper limb training. Non-invasive brain stimulation involves 10 daily sessions of cTBS of the contralesional hand area of the primary motor cortex over a period of 2 weeks. The first cTBS session is executed within 3 weeks after stroke onset. A subset of patients undergoes five MRI sessions to assess post-stroke brain reorganization. The primary outcome measure is the upper limb function score, assessed from grasp, grip, pinch and gross movements in the action research arm test, measured at 3 months after stroke.

Chapter 7 reports the results of a qualitative study that was conducted as part of the BSTARS trial in a specialized rehabilitation center. Data were collected through face-to-face semi- 
structured interviews with 13 stroke patients who completed the 10-day TMS intervention for upper limb recovery. The interviews were recorded, transcribed verbatim and analyzed using thematic analysis. Important components related to the positive experience of the design and delivery of the treatment included comfort (i.e. moment of relaxation) and the sensation of a painless treatment without side-effects. Key concerns included uncertainty and anxiety about possible negative consequences and group allocation. This study demonstrated that TMS is well accepted by stroke patients with an upper limb paresis.

Finally, in Chapter 8 I discuss our main findings in the light of improved understanding and assessment of post-stroke impairment and recovery, with the ultimate aim to optimize recovery, for instance by including non-invasive brain stimulation in the rehabilitation of stroke. Further, methodological considerations related to the study population, study design, outcome measures of the used studies are debated. Finally, clinical implications and recommendations for future research are provided. 


\section{Samenvatting (Summary in Dutch)}

Jaarlijks krijgen wereldwijd meer dan 15 miljoen mensen een beroerte. Een beroerte is één van de belangrijkste oorzaken van invaliditeit bij volwassenen. De impact van deze veelvoorkomende en ernstige aandoening is aanzienlijk. Een beroerte leidt vaak tot fysieke en psychologische beperkingen die gevolgen hebben op het dagelijks en sociaal functioneren. Spierzwakte van een arm is één van de meest voorkomende gevolgen, en komt in de vroege fase na de beroerte voor bij meer dan $60 \%$ van de patiënten. Zelfstandig wassen en aankleden, een broodje smeren en naar het toilet gaan zijn dan vaak niet meer vanzelfsprekend. Een verstoorde armfunctie hangt samen met een lagere kwaliteit van leven. Of verbetering van de armfunctie leidt tot verbetering van kwaliteit van leven op de lange termijn was eerder nog niet onderzocht.

Herstel na een beroerte verloopt bij iedere patiënt anders en in een variërend tempo. Vooral in de eerste paar maanden na een beroerte is de kans op herstel het grootst en vindt er verbetering plaats in de motorische functie van de verlamde arm. Een groot deel van de patiënten laat (gedeeltelijk) herstel van de verlamming zien, maar een kwart tot ongeveer één derde van de patiënten herstelt niet tot nauwelijks en behoudt een ernstig aangedane arm- en handfunctie. Daarom is het belangrijk om zo snel mogelijk te starten met revalideren. Voor veel dagelijkse activiteiten is de inzet van beide armen belangrijk. Het uitvoeren en oefenen van activiteiten met twee handen krijgt dan ook veel aandacht tijdens de revalidatie. Aangezien er veel verschillende revalidatiebehandelingen mogelijk zijn (onder andere oefentherapie, spiegeltherapie en elektrostimulatie), kiest de therapeut meestal de behandeling die het beste overeenkomt met de doelen en wensen van een patiënt.

Wetenschappelijk onderzoek naar behandelingen voor het herstel van de armfunctie heeft vooral plaatsgevonden in de chronische stabiele fase na een beroerte (vanaf 6 maanden na de beroerte). De eerste 3 maanden na een beroerte worden vaak gezien als de revalidatiefase, de fase waarin de hersenen zich het beste kunnen herstellen. Helaas ontbreekt er nog goed wetenschappelijk bewijs van het effect van behandelingen die het herstelvermogen van de hersenen kunnen bevorderen. De afgelopen jaren is er groeiende interesse in het gebruik van niet-invasieve hersenstimulatie om het herstel van motorische functie te verbeteren. Het gebruik van niet-invasieve hersenstimulatie in de vroege fase na een beroerte en de langetermijneffecten op armfunctie hebben nog weinig aandacht gekregen in de wetenschappelijke literatuur.

In dit proefschrift wordt gefocust op herstel van armfunctie na een beroerte, en op de mogelijkheden van niet-invasieve hersenstimulatietechnieken, in het bijzonder transcraniële magnetische stimulatie (TMS), om de armfunctie te verbeteren. 
In Hoofdstuk 1 wordt een overzicht van de opbouw van dit proefschrift gegeven. De gevolgen van een beroerte op armfunctie en kwaliteit van leven worden besproken. Daarnaast wordt TMS geïntroduceerd als een neuro-modulerende techniek om herstel van de arm te bereiken. Het monitoren en het evalueren van motorisch herstel wordt ook beschreven.

\section{Deel I Beperkingen en herstel na een beroerte}

In Hoofdstuk 2 hebben we de overeenkomsten beschreven van reikbewegingen met de arm en hand tussen ratten en mensen in de subacute fase na een beroerte. De relatie van de reikbewegingen met uitkomstmaten uit de kliniek werd ook bekeken. De reikbeweging (het reiken naar voedsel op een plateau) is een gedragsmatige methode waarmee een onderscheid gemaakt kan worden tussen werkelijk herstel van armfunctie en compensatie. De taak kan uitgevoerd worden door zowel ratten als mensen, wat van belang is voor translationeel onderzoek (de brug tussen laboratoriumonderzoek en de klinische praktijk). In totaal werden er 12 patiënten geïncludeerd vanuit de B-STARS studie (Brain STimulation for Arm Recovery after Stroke) en 17 ratten vanuit een soortgelijk preklinisch onderzoek. De bewegingspatronen van de reikbeweging lieten significante gelijkenissen zien tussen ratten en mensen. Beiden lieten significante verbeteringen zien op de bewegingselementen over de tijd. Op de eerste meting na de beroerte zagen we zwakte van de spieren en een vertraagd herstel in spieren van de onderarm en hand/poot. Ratten vertoonden bij bepaalde bewegingselementen (bijvoorbeeld semi-gebogen vingers, richten, pronatie en grijpen) vanaf het begin een bijna normale uitvoer, waardoor er geen verbetering over de tijd meetbaar was. De reikbeweging liet sterke samenhang zien met de veel gebruikte uitkomstmaten uit de kliniek in de eerste drie maanden na een beroerte. Concluderend lijkt de reikbeweging een waardevolle en effectieve translationele taak voor het beoordelen van motorisch herstel bij mensen en ratten met een beroerte.

Hoofdstuk 3 richt zich op de vergelijking tussen zelfrapportage en observationele klinische maten voor verbetering van de armfunctie bij patiënten met een beroerte. Het herstel van de paretische arm kan worden beoordeeld door een clinicus (observationeel) of door de patiënt zelf (zelfrapportage). Voor deze studie includeerden wij 159 patiënten met een herseninfarct en een paretische arm. Het tijdsbestek na een beroerte (0-3 of 3-6 maanden) leek geen invloed te hebben op de overeenkomst tussen de observationele en zelf-gerapporteerde metingen: er waren meer overeenkomsten dan verschillen tussen de metingen. In het geval van een verschil tussen de metingen waren de zelfrapportages pessimistischer (86\%) in de eerste 3 maanden na beroerte in vergelijking tot de 3 maanden daarna (39\%). Zelf-gerapporteerde metingen kunnen worden gebruikt als aanvulling op observationele metingen om het herstel van de arm te beoordelen. Informatie over het 
vermogen en het gebruik van de paretische arm buiten de behandeling is waardevol voor clinici, omdat het meer inzicht geeft in het perspectief van de patiënt.

In Hoofdstuk 4 hebben we gekeken naar de langetermijnrelatie tussen herstel van armfunctie en veranderingen in kwaliteit van leven na ontslag uit een klinische revalidatiesetting. In totaal konden er 250 patiënten uit de FIT-Stroke studie worden geïncludeerd. Deze patiënten waren onderzocht bij ontslag uit de klinische revalidatiesetting en 12 weken na het ontslag. De resultaten van onze studie laten zien dat armfunctie (bij ontslag uit een revalidatiesetting) een belangrijke voorspeller is voor kwaliteit van leven 3 maanden later. Een verbetering in kwaliteit van leven is positief gerelateerd aan verbeteringen in armfunctie. Het is dus belangrijk om de behandeling van armfunctie ook na ontslag uit een revalidatiesetting voort te zetten. Het monitoren en behandelen van armfunctie in deze periode kan bijdragen aan een hogere kwaliteit van leven voor deze patiënten.

Deel II Streven naar optimaal herstel - niet-invasieve hersenstimulatie in de revalidatie na een beroerte

De bevindingen van een systematische review van de literatuur over en een meta-analyse van de timing van het inzetten van repetitieve TMS (rTMS) voor verbetering van de armfunctie na een beroerte, zijn beschreven in Hoofdstuk 5. In totaal voldeden er 38 studies, met 1.074 patiënten met een beroerte, aan de inclusiecriteria en konden deze worden geïncludeerd in de meta-analyse. De timing van het starten van de behandeling na een beroerte was op de volgende manier gecategoriseerd: acuut tot vroeg subacuut ( $<1$ maand), vroeg subacuut (1-3 maanden), laat subacuut (3-6 maanden) en chronisch ( $>6$ maanden). rTMS bleek effectief als behandeling te zijn wanneer deze werd gestart binnen de eerste maand na een beroerte, maar niet als de behandeling aanving in de vroege subacute fase of chronische fase na een beroerte. Dit voordelige effect werd alleen gevonden voor uitkomstmaten die meten op het niveau van functie (gemeten met de Fugl-Meyer Arm score), maar niet op het niveau van activiteit. De rTMS studies die zijn uitgevoerd met patiënten met een beroerte waren echter heterogeen, met variërende uitkomstmaten en relatief kleine groepsgroottes. Daarom moeten de resultaten met (enige) voorzichtigheid worden geïnterpreteerd. Op basis van de bevindingen werd de aanbeveling gedaan om een gestandaardiseerde set van metingen te ontwikkelen voor het testen en evalueren van armfunctie.

Hoofdstuk 6 beschrijft het studieprotocol van het klinische B-STARS onderzoek (Brain STimulation for Arm Recovery after Stroke). De primaire onderzoeksvraag van de studie, die tijdens het schrijven van dit proefschrift nog lopende was, is het vaststellen van het therapeutische effect van contralaterale continuerende inhiberende theta burst stimulatie (cTBS) op het herstel van de paretische arm 3 maanden na een beroerte. B-STARS is een prospectief, 
gerandomiseerd, dubbelblind gecontroleerd klinisch onderzoek. Deelnemende patiënten worden gerandomiseerd ingedeeld in een 'echte' of placebo hersenstimulatiegroep, wat wordt gevolgd door een training voor de arm in de standaardzorg. Niet-invasieve hersenstimulatie bestaat uit 10 dagelijkse sessies van cTBS van het contralaterale handgebied van de primaire motorische cortex over een periode van 2 weken. De eerste cTBS-sessie wordt binnen 3 weken na de beroerte uitgevoerd. Optioneel kunnen de patiënten vijf MRI-scans ondergaan, zodat de hersenreorganisatie beoordeeld kan worden. De primaire uitkomstmaat is een armfunctiescore, die de grijp-, grip-, knijp- en grove bewegingen beoordeelt (action research arm test), die wordt gemeten drie maanden na de beroerte.

Hoofdstuk 7 laat de resultaten zien van een kwalitatieve studie die is uitgevoerd als onderdeel van de B-STARS trial in een gespecialiseerd revalidatiecentrum. De data zijn verzameld door semigestructureerde face-to-face interviews met 13 patiënten met een beroerte die de 10-daagse TMS-interventie voor het herstel van de arm hadden voltooid. De interviews werden opgenomen, getranscribeerd en geanalyseerd middels thematische analyse. Belangrijke componenten die gerelateerd zijn aan de positieve ervaring met het design en het ondergaan van de behandeling zijn het comfort (moment van ontspanning) en de sensatie van een pijnloze behandeling zonder bijwerkingen. Belangrijke zorgen waren de onzekerheid en angst over mogelijke negatieve consequenties en de groepsindeling (wel of geen placebo). Deze studie toont aan dat TMS goed wordt ontvangen door beroertepatiënten met een armparese.

Tenslotte worden in Hoofdstuk 8 de belangrijkste bevindingen samengevat en besproken. De focus ligt daarbij op het begrijpen en het evalueren van beperkingen en herstel na een beroerte, met als uiteindelijk doel het streven naar optimaal herstel - door bijvoorbeeld het gebruik van niet-invasieve hersenstimulatie. Vervolgens worden in dit hoofdstuk de methodologische overwegingen bediscussieerd in relatie tot de onderzoekspopulatie, de onderzoeksopzet en de uitkomstmaten van de gebruikte studies. Tot slot worden de klinische implicaties en aanbevelingen voor toekomstig onderzoek gegeven. 
Dankwoord 

"If you want to go fast, go alone. If you want to go far, go together." - African proverb

Ik wil graag iedereen bedanken die direct of indirect betrokken is geweest bij mijn promotietraject de afgelopen jaren.

\section{De deelnemers}

Allereerst alle patiënten die tijd en energie hebben besteed door deelname aan de B-STARS studie. Zonder jullie bereidheid tot deelname was dit proefschrift er niet geweest. Daarnaast ook dank voor de mooie, ontroerende gesprekken en humor tijdens de behandelingen en metingen.

\section{Promotiecommissie}

Prof. dr. Dijkhuizen, beste Rick, ik wil je in het bijzonder bedanken voor de uitvoerige begeleiding en feedback, waardoor ik mijn onderzoeks- en schrijfvaardigheden heb kunnen verbeteren en ontwikkelen in de afgelopen jaren. Ik bewonder je optimisme waarmee je de wetenschap bedrijft en elke klus kan en wil klaren. Naast de wetenschappelijke discussies was er ook tijd voor discussies over de uitspraak van woorden en sportieve uitdagingen. Het dak van het hotel in Barcelona beklimmen en de felle discussies over voltige en de paardensport staan mij nog goed bij. Ik proef ook nog steeds de overwinning van mijn zeer ruime voorsprong van 7-1 in potjes met tafeltennis. Ik ben benieuwd of er nog een comeback komt.

Prof. dr. Visser-Meily, beste Anne, bedankt voor je vertrouwen, begeleiding en betrokkenheid tijdens mijn promotietraject. Het is inspirerend om jouw werklust en onuitputtelijke inzet te zien voor de revalidatiegeneeskunde, en hoe je op de hoogte bent van zowel de klinische als wetenschappelijke ontwikkelingen. Je bent altijd bereikbaar voor vragen of een praatje wat ik als heel prettig en warm heb ervaren. Zo kreeg ik zelfs een keer de uitnodiging om te brainstormen bij jou aan de keukentafel over een artikel terwijl je een vrije dag had. En dank voor de kansen die ik heb gekregen om als psycholoog in de Revalidatiegeneeskunde te werken.

Dr. van der Worp, beste Bart, wat fijn dat je onderdeel bent van ons team. Jouw kennis en ervaring met het doen van klinische trials zijn van enorme waarde en daar heb ik veel gebruik van kunnen maken. Daarnaast was je altijd bereikbaar voor spoedvragen over patiënten, dilemma's en grapjes. Naast serieuze zaken werd er namelijk ook heel veel gelachen tijdens de B-STARS overleggen. 
Dank aan alle leden van de beoordelingscommissie, dr. Carel Mesker, Prof. dr. Cindy Veenhof, Prof. dr. Chris Dijkerman, Prof. dr. Jaap Kappelle en Prof. dr. Sander Geurts, voor de tijd en energie voor het lezen en beoordelen van mijn proefschrift.

\section{Onderzoeksteam}

Graag wil ik ook alle co-auteurs op de artikelen in dit proefschrift bedanken voor de samenwerking. Beste Adam Pel, Bas Neggers, Caroline van Heijningen, Geralda van Tilborg, Gert Kwakkel, Ingrid van der Port, Lilliane Jacobs, Jord Vink, Julia Boonzaier, Maaike Pelsma, Roel van Hooijdonk, Rinske Nijland, Tanja Nijboer, dank jullie wel voor het meedenken, de input en de constructieve feedback.

Beste Jord, ik waardeer het enorm dat je in het B-STARS-team bent gestapt. Ondanks onze niet volledig op elkaar aansluitende stijl van werken, hebben we steeds beter onze krachten weten te bundelen en hebben we heel fijn kunnen samenwerken. Daarnaast konden we elkaar ook vinden voor aanbevelingen voor nummers, filosofische gesprekken en goede koffie aan de overkant. Ik vind het mooi hoe jouw blik op de meerwaarde voor de patiënt gericht blijft tijdens het doen van onderzoek.

De stagiaires, zonder jullie was de uitvoer van de B-STARS-studie onmogelijk geweest. Adam Pel, Beatriz Gouveia, Frederieke Smeitink, Hosna Anwar, Ida van de Water, Lilliane Jacobs, Lisette Peters, Mariëlle Grosveld, Marissa Riemens, Marit de Jong, Marius Sauca, Marloes Lugtenberg, Roosmarijn Brenninkmeijer, Rosa Callenfels, Sjors Heuberger, bedankt voor jullie inzet, enthousiasme en betrokkenheid bij het onderzoek.

Artsen, behandelaars, behandelplanners en teammanagers van De Hoogstraat Revalidatie, Annet Slabbekoorn, Ellen Agterhof, Esther van leren, Frank van Donselaar, Heidy Jaspers, José Hooijer, Karlijn Mulder-Bouwens, Lidian Handstede, Manin Konijnenbelt, Marike Jansen, Mirjam Kouwenhoven, Oscar Haver, Patricia Passier, Tom Lenaers, Wilma Jentink, dank voor jullie medewerking, het meedenken en de inzet bij de opzet en het verloop van de B-STARS-studie. Behandelplanners, voornamelijk Alice van der Tol, Debby van Rijn, Dian Baljet en Lidian Handstede, wil ik bedanken voor de flexibiliteit en het snelle handelen. In het bijzonder wil ik Eugenie en Cecile bedanken. Jullie stonden altijd in de startblokken als ik vragen had en/of hulp nodig had. De fijne kneepjes van het afnemen van de motorische taken (oefenen met de Fugl-Meyer) en kennis over de handengroep, transfers en neurorevalidatie heb ik van jullie geleerd. Ik bewonder jullie positiviteit, aanstekelijke enthousiasme en professionaliteit. Ik kijk nog met veel plezier terug op ons werktripje naar Maastricht en de treinrit naar huis gevuld met gelach, geroddel, Turkse pizza en aluminiumfolie. Dank voor jullie vertrouwen in mij. 
Behandelaren en divisie Beeld medewerkers van het UMC Utrecht. Beste Ben Fengler, dank voor je flexibiliteit, inzet, enthousiasme en alle ARAT-afnames op de specifieke momenten. Beste Alex Bhogal, Fredy Visser, Hans Hoogduin, Jeroen Hendrikse, Mathijs Raemaekers, Niels Blanken en laboranten, dank voor alle MRI-gerelateerde antwoorden, acties, scans en hulp.

\section{Collega's}

Mijn collega's van het Kenniscentrum Revalidatiegeneeskunde: de senioren Anita, Marcel, Marjolijn en Olaf, de junioren Eline, Heleen, Ilona, Isabel, Jessica, Joris, Joris, Lauriane, Maremka, Mattijs, Mette, Maren, Nicole, Teuni, Remko, Tanja, Tijn, Vincent, dank jullie wel voor de gezelligheid, borrels, de frustratie eruit slaan met tafeltennis en badminton, koffietjes, estafette in een panter-legging, wandelingen door het park en uitjes. Lieve Raquel, van fijne collega naar één van mijn beste vriendinnen. Naast urenlange gesprekken over werk(druk), presteren en schrijven hebben we elkaar ook gevonden in veel drankjes doen en pizza's eten na het werk. Dank je wel voor alle mooie en hilarische momenten en wat hebben we wat werkstress van ons af gedanst in de nachtelijke uurtjes op feesten met tropische deuntjes. Carlijn, wat ben je onmisbaar voor ons. Dank voor al het enthousiasme waarmee we samen heel veel uitjes hebben georganiseerd, met als kers op de taart ons eigen wie-is-de-mol spel.

Natuurlijk ook dank aan mijn collega's van het 'dierenlab', Annette, Anu, Bart, Caroline, Esther, Geralda, Gerard, Julia, Michel, Milou, Tessa, Vera, Wim, dank voor alle leuke momenten samen waaronder de nieuwjaars- en paasontbijtjes, teamuitjes, sinterklaasvieringen en tafeltennistoernooien.

Mijn nieuwe collega's bij OCA in Amersfoort, beste Anja, Auke, Ben, Larissa en Maarten, wat hebben we een fijn team. Dank voor jullie interesse en vertrouwen in mij. Auke en Maarten, mijn agenda hou ik vrij voor alle 'bescheiden' avonden die nog gaan komen.

\section{Vrienden en familie}

Mijn vrienden uit Almere, dank voor alle in- en ontspanning en dat jullie er zijn! Lieve Aylene, bedankt voor het gouden vertrouwen in mij. Als er iemand zo complimenteus is over werkelijk, ja, werkelijk alles, wat ik doe ben jij het wel. De afgelopen jaren waren voor ons letterlijk en figuurlijk bloed, zweet, tranen (in positieve zin) en schaterlachen en ik ben ontzettend blij dat we dit allemaal samen delen. Ik wil je bedanken voor je lieve woorden en vriendschap. Je bent geweldig en wat fijn dat je mijn paranimf bent!

Lieve Paul en Deveney, dank voor alle leuke uitjes (inclusief de snelweg!), goede gesprekken, spelletjes, eetfestijntjes, wat een verwennerij! Lieve Guido, dank voor alle vurige discussies en filosofische (ambitie)gesprekken, espresso's en fanatieke sport- en dansmomenten. Lieve 
Sabine, de andere hamster die mijn energieniveau snapt en omarmt. Ik waardeer je humor, oprechtheid en gezelligheid!

Vriendinnen uit Leiden (en omstreken), lieve Ilona, Jolanda, Jolien, Joyce, Maartje, Maxime, Maxine, Tineke, dank voor de prachtige studietijd en het studentenleven in Leiden. In het bijzonder, Kirsten, dank voor alle lieve woorden en oprechte interesse. Volgens mij raken wij nooit uitgepraat; zij het aan de churros (terwijl we commissietaken hadden) in Madrid, de nachtelijke uurtjes in de lobby van ons Indiase hotel in een achterstandswijk in New Delhi of borreltijd in een wijk in Rome waarvan we de naam nog steeds verkeerd uitspreken. En wat bijzonder dat we onze passie voor psychologie en behandelen delen en hier elkaar weten te vinden, fascineren en helpen.

Lieve Open Air groep, ik ben door jullie met open armen ontvangen. Wat heb ik genoten van alle festivals, feestjes, etentjes, campingtripjes, picknicks. We hebben heel wat gemist door Corona, maar laten we alles driedubbel inhalen.

Lieve Hugo en Mathijs, wat een avonturen hebben we beleefd in Indonesië, toen ik nog aan het begin van mijn promotieavontuur stond. Dank voor jullie vrolijkheid, humor en interesse. Hugo, hoe leuk dat we samen deze prachtige omslag hebben gemaakt. Heel veel dank! Lieve Cheryl, van één korte, maar krachtige week in Jakarta naar ondertussen jaren vriendschap. Ik bewonder je nieuwsgierigheid, enthousiasme en stiekem waardeer ik ook je preken over ontspannen en op tijd naar bed gaan.

Mijn vriendinnen uit Utrecht, lieve Margriet, Nicole en Veerle (+ Loet). In de beruchte Neudeflat begon onze vriendschap op een penthouseborrel. Op sokken naar elkaar toe gaan, of je eigen bord meenemen, is nu wat minder praktisch, maar de koffietjes, etentjes, picknicks, en Zoom-bootcamps zijn er niet minder gezellig door geworden. Dank jullie wel voor jullie aanmoedigingen en betrokkenheid.

Lieve Martina en Ton, Els en Erwin, dank voor alle mooie momenten en interesse. Martina, dank voor alle wijze tante woorden en verhelderende gesprekken. Ik kijk uit naar het pellen van amandelen en lampenolie drinken in Spanje.

Lieve schoonfamilie, dank voor het warme welkom in jullie gezin, jullie gastvrijheid en betrokkenheid.

Lieve pap en mam, bedankt voor jullie onvoorwaardelijke liefde, gezelligheid en steun. Ik heb veel bewondering voor jullie doorzettingsvermogen, leergierigheid en aandacht voor anderen; jullie zijn hierin een voorbeeld voor mij. Bedankt voor jullie vertrouwen in mij, de eeuwige interesse en de vrijheid om mijn eigen keuzes te maken. De avonden waarbij we met $z$ 'n allen springend en brullend aan de eettafel zitten en dansen in de woonkamer zullen nooit vervelen. 
Lieve Anne. Mijn kleine zusje waar ik ongelooflijk trots op ben. Ik bewonder je avonturisme en kracht. Dank je wel voor wie je bent en dat je altijd aan mijn zijde staat. Naast mijn zusje ben je ook één van mijn beste vriendinnen. Wat een geluk dat we onze zeer specifieke humor, vreemde woorden, gekke buien, reis- en (vr)eetlust bij elkaar kwijt kunnen. Wat fijn dat je als paranimf naast mij staat! Lieve Joshua, met jouw leuke, interessante vragen en warme persoonlijkheid ben je een aanwinst voor de familie. Ik bewonder hoe jij in het leven staat! Lieve Jochem. De woorden hier zullen niet alles dekken met wat ik tegen je wil zeggen. Van een collega op het werk, en in dezelfde commissie (bedankt Joris), werd je iets meer (okay, heel wat meer) dan een collega. Naast samen stuiterballen en veel avonturen wil ik je bedanken voor je motiverende speeches, wijze woorden en dit-is-pas-het-begin-van-jecarrière peptalk als ik door de bomen het bos niet meer zag. Daarnaast heb ik de kunst van het ontspannen bij jou kunnen afkijken, en heel vaak kandidaat mogen spelen voor jouw professionele massages. Wat bof ik met jou! Ik hou van je en kan niet wachten om straks bij jouw promotie naast je te staan! 
About the author 



\section{Curriculum vitae}

Eline van Lieshout was born on February the $5^{\text {th }} 1993$ in Almere, the Netherlands. In 2011 she finished secondary school at Helen Parkhurst in Almere (VWO). Thereafter, she studied Psychology at Leiden University. After obtaining her Master's degree (cum laude) in Clinical Neuropsychology in 2015, she traveled to Jakarta, Indonesia to teach Biological Psychology at the Atma Jaya University. In March 2016 she started as a PhD student at the UMC Utrecht and at De Hoogstraat Rehabilitation, which has resulted in this thesis.

Since February 2020 Eline is working as psychologist, in addition to her $\mathrm{PhD}$, in the specialized medical rehabilitation. From the summer of 2020 Eline also started working as psychologist doing neuropsychological examinations and treating patients with acquired brain injury for the rehabilitation

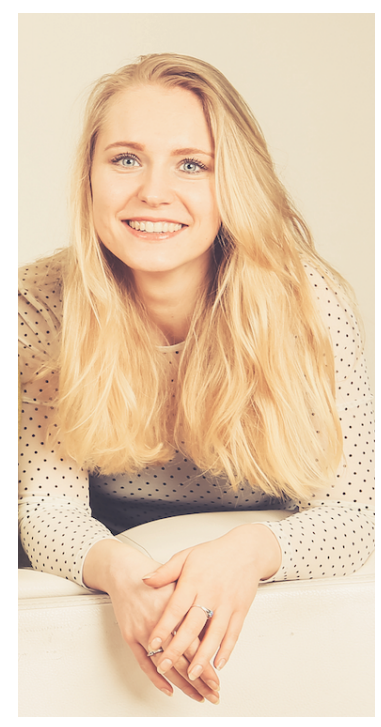
medicine and sports department of the University Medical Center Utrecht. In 2021 Eline will continue in her position as psychologist in the specialized medical rehabilitation, start at PSYTREC (psychotrauma expertise clinic), and continuing scientific work at the University Medical Center Utrecht. 


\section{List of publications}

\section{International publications}

van Lieshout ECC, Visser-Meily, JMA, Neggers, SFW, Worp, HB, Dijkhuizen, RM. Brain stimulation for arm recovery after stroke (B-STARS): protocol for a randomized controlled trial in subacute stroke patients. BMJ Open 7, e016566 (2017).

van Lieshout ECC, van Hooijdonk RF, Dijkhuizen RM, Visser-Meily JMA, Nijboer TCW. The effect of non-invasive brain stimulation on post-stroke cognitive function: a systematic review. Neurorehabil. Neural Repair 33, 355-374 (2019).

van Lieshout ECC, van der Worp HB, Visser-Meily JMA, Dijkhuizen RM. Timing of repetitive transcranial magnetic stimulation onset for upper limb function after stroke: a systematic review and meta-analysis. Front. Neurol. 10, 1269 (2019).

van Lieshout ECC, van de Port IG, Dijkhuizen RM, Visser-Meily JMA. Does upper limb strength play a prominent role in health-related quality of life in stroke patients discharged from inpatient rehabilitation? Top. Stroke Rehabil. 27, 525-533 (2020).

van Lieshout ECC, Visser-Meily JMA, Nijland RH, Dijkhuizen RM, Kwakkel G. Comparison of self-reported versus observational clinical measures of improvement in upper limb capacity in patients after stroke. J. Rehabil. Med. 52, jrm00051 (2020).

van Lieshout ECC, Jacobs LLJ, Pelsma M, Dijkhuizen RM, Visser-Meily JMA. Exploring the experiences of stroke patients treated with transcranial magnetic stimulation for upper limb recovery: a qualitative study. BMC Neurol. 20, 365 (2020).

van Lieshout ECC, Boonzaier J, Pel AJ, van Heijningen CL, Vink JJ, Visser-Meily JMA, van Tilborg GAF, Dijkhuizen RM. Translational value of skilled reaching assessment in clinical and preclinical studies on motor recovery after stroke. Neurorehab. Neural Repair. [under review]

\section{National publications}

van Lieshout ECC, Visser-Meily JMA, van der Worp HB, Neggers SFW, Slabbekoorn AJH, Dijkhuizen RM. Geef mij maar hersenschokjes. Nederlands Tijdschrift voor Revalidatiegeneeskunde, April 2018. 

UMC Utrecht ISBN 978-90-393-7367-5 